\author{
Universidade de São Paulo \\ Instituto de Física
}

\title{
Espectroscopia de correlação em condição de Transparência Eletromagneticamente Induzida: regime dinâmico e ressonâncias das bandas laterais em átomos frios
}

\author{
Hans Marín Flórez
}

Orientador:

Prof. Dr. Marcelo Martinelli

Tese de Doutorado apresentada ao

Instituto de Física para a obtenção do título de Doutor em Ciências.

Banca Examinadora:

Prof. Dr. Marcelo Martinelli (IFUSP)

Prof. Dr. José Tito da Luz Mendonça (IST)

Prof. Dr. Arturo Antonio Lezama Astigarraga (Universidad de la Republica)

Prof. Dr. Philippe Wilhelm Courteille (IFUSP-São Carlos)

Prof. Dr. Arnaldo Gammal (IFUSP)

São Paulo 


\section{Resumo}

Estudamos a espectroscopia de correlação de intensidade de dois feixes laser com excesso de ruído de fase em condição de transparência eletromagneticamente induzida (EIT) em átomos frios de rubídio. Em particular, analisamos três aspectos da espectroscopia de correlação. O primeiro, no âmbito teórico, corresponde ao tratamento perturbativo da dinâmica atômica para o cálculo do espectro de correlação. Mostramos que, para campos com baixo ruído de fase, o termo a primeira ordem da expansão é suficiente para descrever completamente a correlação do sistema. No entanto, para campos que apresentam excesso de ruído de fase, observamos que é necessário considerar os termos de ordem superior para obtermos uma descrição completa do sistema. A abordagem perturbativa nos permite ter uma expressão analítica que relaciona o espectro de correlação no domínio das frequências com as propriedades de absorção e dispersão associadas ao meio atômico.

No âmbito experimental, analisamos duas situações diferentes para a espectroscopia de correlação. A primeira foi a espectroscopia de correlação para diferentes valores de dessintonia de frequência com relação à ressonância atômica. Mostramos pela primeira vez que a correlação apresenta ressonâncias na frequência das bandas laterais enquanto os campos estiverem dessintonizados fora da largura espectral dada pela emissão espontânea. Nossos resultados experimentais são descritos de maneira satisfatória pelo modelo perturbativo para a correlação definida no domínio da frequência. Além disso, mostramos que em certas condições, o modelo perturbativo se reduz ao nosso modelo heurístico para a função de correlação $g^{2}(0)$ no domínio do tempo, sendo ela uma aproximação válida para descrever correlação entre os campos. 
O terceiro aspecto estudado neste trabalho se refere aos efeitos dinâmicos sobre a espectroscopia de correlação. Investigamos como a evolução transiente do sistema atômico, devido às forças de espalhamento que a luz exerce sobre os átomos, induz a uma assimetria espectral na correlação, se apresentando assim, como um efeito de "histerese". No entanto, para campos dentro da janela de transparência, mostramos que o sistema é insensível ao efeito de tais forças. Esta é a primeira observação do efeito dinâmico sobre a espectroscopia de correlação. Assim, mostramos que a espectroscopia de correlação se torna um ferramenta de alta precisão para detectar efeitos dinâmicos nos átomos frios, pois tais efeitos escapam da simples observação quando é feita a espectroscopia de transmissão padrão. 


\begin{abstract}
We studied the intensity correlation spectroscopy of two lasers beams with excess of phase noise in electromagnetically induced transparency (EIT) condition in cold rubidium atoms. In particular, we analyzed three aspects of correlation spectroscopy. In the first one, as a theoretical approach, I proposed a perturbative treatment for the atomic dynamics in order to calculate the correlation spectrum. We show that for electromagnetic fields with low phase noise, the first order term of the expanssion is sufficient for a complete description of the system. However, when the fields present excess of phase noise, it is shown that higher order terms are necessary to describe the system completely. The perturbative approach allows the contruction of an analytical expression which maps the correlation spectrum in the frequency domain, with the absorptive and dispersive properties of the atomic medium.
\end{abstract}

In the experimental part, we analyze two different situations for the correlation spectroscopy. In the first one, was the correlation spectroscopy for different values of frequency detuning with respect to the atomic resonance. We show for the first time that the correlation has resonances at the exactly sideband's frequencies, if the light fields optical frequencies are detuned from the natural spectral width given by the spontaneous emission. Our experimental results are described satisfactorily by the perturbative model for the correlation set in the frequency domain. Furthermore, we show that under certain conditions, the perturbative contains our heuristic model for the correlation function $g^{2}(0)$ in the time domain, and it is a valid approximation to describe correlation between fields.

The third aspect studied in this work refers to the dynamical effects on the correlation spectroscopy. We investigate the transient evolution of the atomic system due to the scatte- 
ring forces that light exerts on the atoms, inducing a spectral asymmetry in the correlation, appearing as an "hysteresis effect". However, for fields within the transparency window, we show that the system is insensitive to the effect of such forces. This is the first observation of the dynamical effect in the correlation spectroscopy. Thus, we show that correlation spectroscopy becomes a high-precision tool to detect dynamical effects in cold atoms, because such effects escape from the simple observation in the standard transmission spectroscopy. 


\section{Agradecimentos}

Agradeço primeiramente ao Prof. Marcelo Martinelli pelo apoio e orientação durante este trabalho. A sua dinâmica de orientação me deu o espaço e a motivação suficientes para propor soluções e trazer novas ideais à pesquisa desenvolvida durante meu doutorado. Parte da confiança que adquiri durante esse período se deve a ele, e é com ela que caminho agora para desenvolver meu trabalho como pesquisador.

Ao Prof. Paulo Nussenzveig, com quem aprendi a identificar os elementos chaves para enfrentar um problema na pesquisa, principalmente quando nos deparamos com problemas que parecem superar nossos conhecimentos.

À minha família. À minha esposa Ana, minha companheira, e quem me motiva sempre a olhar a física de outro ponto de vista. À meu pai e minha mãe, por sempre me mostrarem que as responsabilidades adquiridas e os sacrifícios no transcurso da vida tem suas gratas recompensas. Esse trabalho é uma delas. Gracias a su apoyo incondicional. Ao meu irmão, cuja voz da experiência me guiava para traçar o meu próprio caminho.

Aos meus colegas do laboratório Ashok, Klara e Carlos, com que formamos uma equipe e com quem sofremos para desvendar alguns dos problemas que nos tomou bastante tempo para resolver.Também agradeço a todos aqueles que se lançaram as primeiras experiências para medir correlação em EIT, em particular a Daniel Felinto pelas discussões ao longo desse periodo e a Arturo Lezama, cuja colaboração com o grupo permitiu dar as bases desse trabalho. Agradeço também a Vanderlei Bagnato por nos fornecer o Rubídio no momento em que precisávamos.

Aos meus amigos Oscar, Carlos Gonzalez, Carlos Mera, Daniel "el paisa", Diana, Gina, David "el mexicano", pelos gratos momentos e com os quais discutimos tanto sobre as nos- 
sas experiências aqui no Brasil.

Agradeço ao Eber e à Paula, da secretaria da CPG, e ao Luiz e à Edi, da secretaria da FEP, pelo trabalho competente e à disposição de sempre ajudar.

Por fim, agradeço imensamente à FAPESP por seu apoio financeiro durante este trabalho. 
Ao meu filho João J.M. 



\section{CONTEÚDO}

Introdução

$\mathbf{V}$

I Tópicos sobre os campos eletromagnéticos e a sua interação com a matéria

1 Descrição do campo eletromagnético 3

1.1 Descrição clássica do campo eletromagnético . . . . . . . . . . . . . . . 4

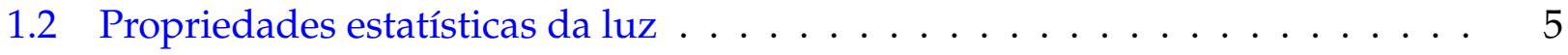

1.2.1 Função de correlação de primeira ordem . . . . . . . . . . . . . . 6

1.2.2 Função de correlação $g^{(2)}(\tau) \ldots \ldots \ldots \ldots \ldots$. . . . . . . . 12

1.2.3 Densidade espectral do campo $S(\omega) \ldots \ldots \ldots \ldots \ldots \ldots$

1.3 Descrição do campo com flutuações de fase estocástica . . . . . . . . . . . 19

1.3.1 Equação de Langevin . . . . . . . . . . . . . . . . . . . . . 20

1.3.2 Dinâmica de variáveis estocásticas: abordagem mediante cálculo de Itô 23

1.3.3 Exemplo: campo elétrico com excesso de ruído na fase $\ldots \ldots . . .28$

1.4 Tópicos da descrição quântica do campo . . . . . . . . . . . . . . . . . . . . . . 29

1.4 .1 Shot-noise . . . . . . . . . . . . . . . . . . . 34 
2 Interação entre a luz e a matéria $\quad 39$

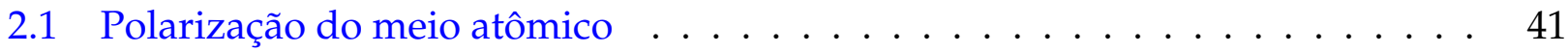

2.2 Descrição microscópica: Matriz densidade . . . . . . . . . . . . . . . . . . 43

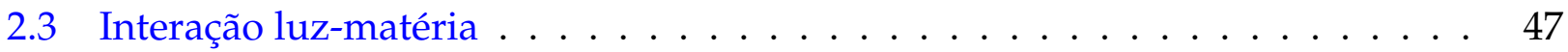

2.3.1 Hamiltoniano mediante interação dipolar . . . . . . . . . . . . . 47

2.3.2 Dinâmica do sistema átomo-campo: equações de Bloch . . . . . . . . . 51

2.4 Força devido à interação luz-átomos . . . . . . . . . . . . . . . . . . . 52

2.4.1 Força em um sistema de 2 níveis . . . . . . . . . . . . . . . . 53

\section{Espectroscopia de correlação em transparência eletromagneticamente induzida

3 Aspectos teóricos das flutuações da luz em condição de Transparência Eletromagneticamente induzida

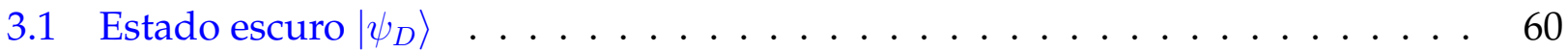

3.2 Susceptibilidade dielétrica linear: condição de EIT . . . . . . . . . . . . . . . 62

3.3 Transformação de ruído de fase para ruído de amplitude (RF-RA) … . . . 67

3.4 Modelo heurístico para correlação $g^{(2)}(0)$ para EIT . . . . . . . . . . . . 78

3.5 Dinâmica do sistema átomo-campo com fase estocástica mediante cálculo de

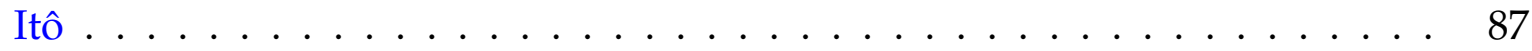

3.5.1 Matriz de Covariância das variáveis atômicas . . . . . . . . . . . 92

3.5.2 Covariância das intensidades dos campos . . . . . . . . . . . . . . 96

3.5.3 Matriz espectral $S(\omega)$ : Solução de Itô . . . . . . . . . . . . . . . . 97

3.5.4 Características da espectroscopia de correlação . . . . . . . . . . . . . . 99

3.6 Resultados para a dinâmica átomo-campo mediante o método perturbativo . 103

3.6.1 Método perturbativo . . . . . . . . . . . . . . . . . . . . . 103

3.6.2 Mapeamento da absorção e dispersão com o espectro de correlação . . 107

3.6.3 Alargamento da espectroscopia de correlação devido às ressonâncias das bandas laterais . . . . . . . . . . . . . . . 118 
4 Resultados experimentais da espectroscopia de Correlação em condição de EIT 123

4.1 Montagem experimental . . . . . . . . . . . . . . . . . . . . . 124

4.1 .1 Átomos frios . . . . . . . . . . . . . 126

4.1 .2 Deteção da luz . . . . . . . . . . . . . . . . . . . 127

4.1.3 Análise do ruído do Laser de diodo mediante rotação de elipse . . . 129

4.1.4 Sequência temporal do experimento . . . . . . . . . . . . . . 134

4.2 Aplicando dessintonia de um fóton na espectroscopia de correlação . . . . . . 136

4.2.1 Ressonâncias $\delta= \pm \omega_{a}$ na espectroscopia de correlação . . . . . . . . . 141

4.2.2 Alargamento por potência das ressonâncias nas bandas laterais . . . . 144

4.3 Forças de espalhamento na espectroscopia de correlação . . . . . . . . . . . . 151

4.3.1 Assimetria do espectro de correlação com respeito a varredura de frequência152

4.3.2 Efeito Doppler induzido pelas forças de espalhamento da luz sobre

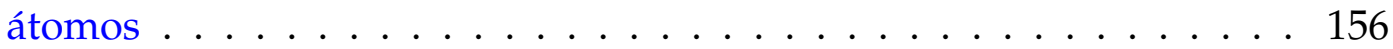

4.3.3 Correlação em geometria contra-propagante . . . . . . . . . . . 160

5 Conclusões e perspectivas $\quad 165$

$\begin{array}{ll}\text { A Expansão perturbativa dos espectros de ruído dos campos } & 171\end{array}$

B Análise teórica da dinâmica de um sistema de cinco níveis 173

B.1 Evolução temporal do sistema de cinco níveis . . . . . . . . . . . . . . . . . 175

$\begin{array}{lr}\text { Bibliografia } & 188\end{array}$ 


\section{INTRODUÇÃO}

Um dos principais objetivos no contexto de informação quântica e computação quântica é a criação de uma interface coerente entre luz e matéria. Dado que a luz é um dos melhores candidatos para transportar informação, e a matéria a melhor opção para armazenar registros de informação, a interface luz-matéria se torna um elemento chave para desenvolver protocolos de armazenamento e processamento de informação. Tudo isso pode fazer parte de uma rede interconectada para manipulação de informação [1], como mostra a figura 1. Nesse tipo de interfaces é necessário um controle coerente da informação no meio atômico usando a luz. Isto é, no caso de memória quântica, precisamos guardar e fazer leitura de registros no meio físico por meio de luz [1-3]. No caso do processador quântico, é necessário manipular de forma coerente os registros de entrada, para obter uma dada transformação codificada nos registros de saída $[4,5]$. Em ambos os casos, a luz é acoplada à matéria durante um intervalo de tempo finito e depois é redistribuído aos diferentes nodos da rede.

Que tipo de estados serão os que são armazenados nessas interfaces? Podemos codificar informação mediante qubits (bits quânticos) no espaço de Hilbert discreto, que pode ser representado de forma geral como $\alpha|0\rangle+\beta|1\rangle[6,7]$. Este tipo de estados podem ser encontrados nos modos de polarização $| \pm\rangle_{H} \mathrm{e}| \pm\rangle_{V}$, em estados atômicos $|e\rangle$ e $|f\rangle$, etc. Outros tipos de estados são aqueles cujos observáveis possuem um espectro contínuo de autovalores. Um exemplo deles é o estado coerente $|\alpha\rangle$, que se caracteriza por ser o estado da luz descrito pela mecânica quântica mais próximo à definição clássica de onda eletromagnética.

Dentro dessas duas abordagens, a de variáveis discretas (VD) e a de variáveis contínuas 


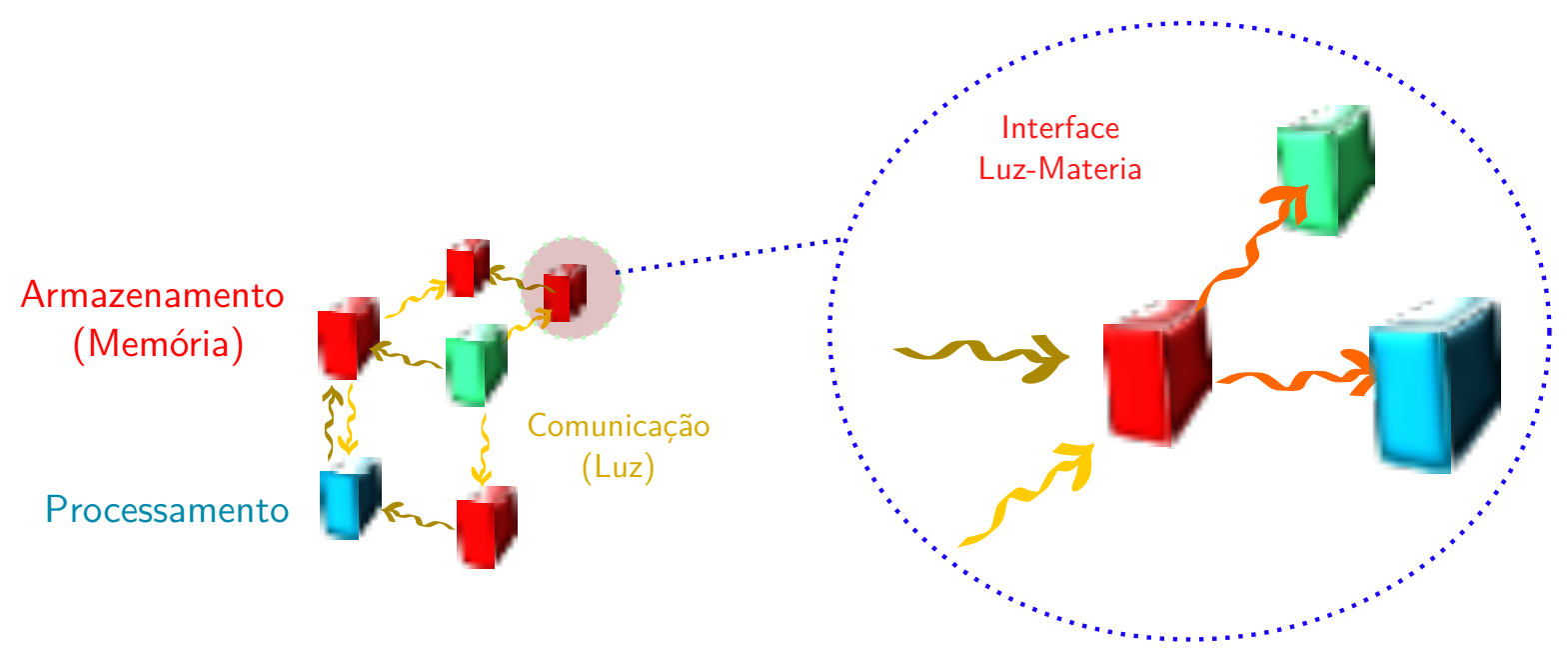

Figura 1: Rede quântica, cujos nodos são interconectados com luz (comunicação), e nos quais é possível armazenar ou processar informação. Abas constituem interfaces de luz-matéria.

(VC), temos um dos grande pilares da informação quântica e da computação quântica: os estados emaranhados $[6,8,9]$. O emaranhamento é uma propriedade particular de sistemas compostos por vários subsistemas de no mínimo duas partes. Este tipo de estado potencializa o processamento quântico, a capacidade criptográfica e a comunicação entre as partes do sistema.

A pesquisa orientada à geração de estados emaranhados e ao armazenamento de informação precisa de uma interface entre a luz e a matéria, em que os fenômenos óticos, lineares ou não lineares, dominam na interação com o meio atômico. Uma das formas de estabelecer essa interface luz-matéria são as memórias quânticas, nas quais se busca armazenamento de luz (que contém informação) [10-17] em meios atômicos, como mostra a figura 2(a). Grandes esforços têm sido feito para aprimorar a eficiência de armazenamento de luz em meio atômicos como, por exemplo, átomos à temperatura ambiente em células de vapor, átomos frios e materiais de estado sólido $[3,18,19])$. Outro tipo de interface são aquelas que exploram a resposta não linear dos meios atômicos, que permitem gerar estados emaranhados [6] ou realizar medidas não destrutivas (QND) [20]. Para geração de estados emaranhados temos, por exemplo, a conversão paramétrica descendente no oscilador paramétrico ótico (OPO) $[21,22]$ e a mistura de quatro ondas (M4O) em átomos à temperatura ambiente[23- 
25]). Entretanto, medidas QND foram mostradas em [26], em que pela interação com o meio atômico, é possível fazer uma medida não destrutiva de uma das quadraturas de um campo sinal $E_{s}$ (em que se codifica a informação), mediante a medida nas quadraturas do campo auxiliar $E_{m}$ (ver figura 2(b)). Na sua saída, parte da informação medida por $E_{m}$ pode ser redistribuída para diferentes nodos da rede quântica da figura 1.

\section{(a)}

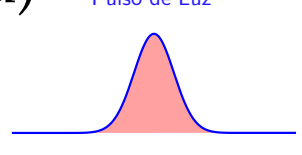

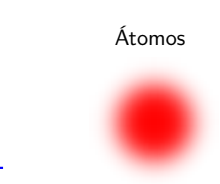
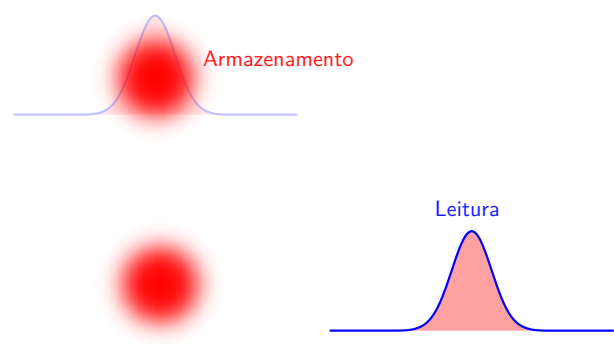

(b)

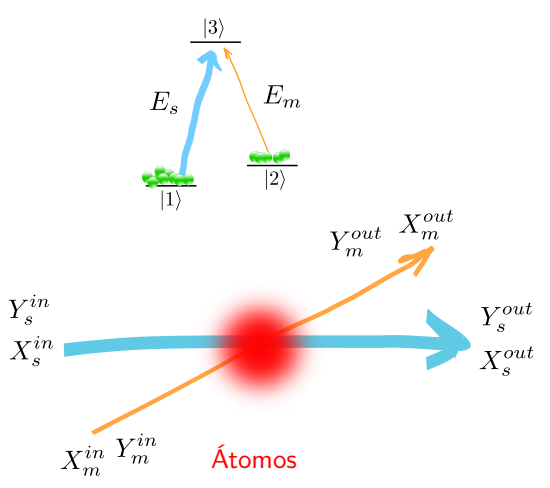

Figura 2: (a) Armazenamento de pulsos de luz em meios atômicos. (b) Medida não destrutiva de uma das quadraturas de um campo sinal $E_{s}$ (em que se codifica a informação), mediante a medida nas quadraturas do campo auxiliar $E_{m}$.

Um dos fenômenos que permite manipular de forma coerente a resposta de meios atômicos, que tem sido amplamente estudado para protocolos de memórias quânticas, é a transparência electromagneticamente induzida (EIT)[18, 27-29]. Nesta interface, dois campos óticos denominados feixe de prova e de controle são acoplados de forma ressonante ao estado excitado de um átomo de três níveis. Devido à interferência destrutiva entre as excitações óticas, o átomo é levado a uma superposição dos níveis fundamentais. Esta superposição de estados, em particular, é denominada estado escuro, e como consequência o estado excitado se desacopla do campo de prova (ver figura 3.(a)). Assim, o meio se torna transparente devido à superposição de estados fundamentais e, portanto, a absorção do meio é nula dentro de uma largura espectral dada por $\Delta \omega_{E I T}$, como mostra a 3.(b). Este fenômeno tem aplicações em protocolos para armazenamentos de pulsos de luz (ver figura 2(a)) durante um intervalo 
de tempo controlado $[1,3,28,30]$. O caso ideal seria atingir o regime de fótons únicos, em que as memórias armazenariam qubits, e assim, funcionariam como repetidores quânticos para distribuir emaranhamento entre dois nodos da rede quântica [30, 31].

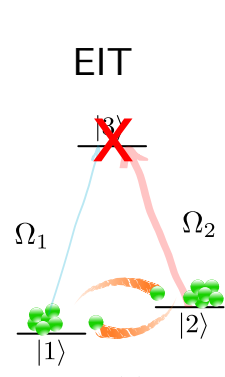

(a)

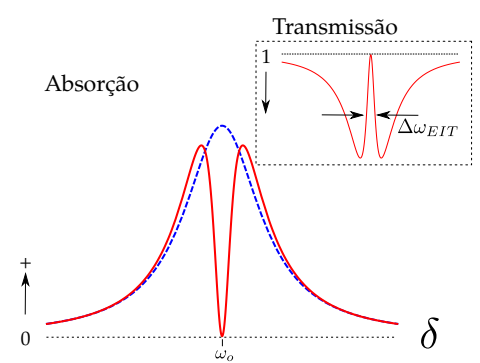

(b)

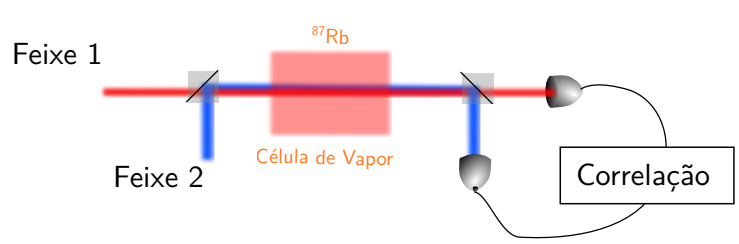

(c)

Figura 3: (a) Interferência de excitações óticas que produzem uma superposição dos estados fundamentais $\alpha|1\rangle+\beta|2\rangle$. (b) Espectros de absorção (transmissão) em função da diferença de dessintonias dos campos $\delta$ (dessintonia de dois fótons). Quando os campos satisfazem a condição Raman $\delta=0$, a absorção é nula, e o meio se torna transparente, dentro de uma largura espectral $\Delta \omega_{E I T}$. (c) Montagem experimental para medir a correlação de intensidade entre dois campos que interagem em condição de EIT com átomos à temperatura ambiente numa célula de vapor.

Enquanto a abordagem de feixes de baixa intensidade (fótons únicos) para uma interface luz-átomos mediante EIT busca implementar os protocolos de armazenamento de luz, a abordagem para feixes intensos pretende estabelecer quais propriedades da luz em condição de EIT apresentam um caráter intrinsecamente quântico, pois foi mostrado que a interferência de excitações presente no fenômeno de EIT não era exclusivamente um efeito quântico, mas tinha um análogo clássico [32,33]. Desenvolveram-se então alguns trabalhos teóricos para estudar verdadeiramente os efeitos quânticos para sistemas átomos-luz em condição de EIT para feixes intensos, por exemplo um estado coerente. Em [17] se mostrou pela primeira vez a possibilidade de se obter estados comprimidos na quadratura de fase para um campo de prova em condição de EIT. Este tipo de efeito é de natureza claramente quântica. Em 2003 começou então um ciclo de pesquisa no nosso laboratório, onde foram feitas as primeiras experiências para medir a correlação entre os campos em EIT usando átomos à temperatura ambiente, observando correlação entre as intensidades dos campos [34] dentro da janela de transparência. A montagem era muito simples, como mostra a figura 3.(c). Dois 
lasers acoplando átomos de três níveis em uma célula de vapor. Após a interação, a intensidade dos campos é medida por fotodetectores, cujos sinais de fotocorrente permitem medir a correlação de intensidade.

A maioria das experiências no regime de feixes intensos eram feitas com laser de diodo, o qual se caracteriza por apresentar um excesso de ruído na sua fase. Portanto, foi necessário estender o mecanismo de conversão de ruído de fase para ruído de amplitude (RF-RA), reportado por Yabuzaki [35], para sistemas de três níveis com campos em condição de EIT. Inspirado nos trabalhos de Hanbury Brown \& Twiss, entre 2005 e 2010, M.O. Scully liderou uma série de trabalhos $[15,36,37]$ adotando uma abordagem temporal. Nesses trabalhos se mediu a função de correlação de intensidade normalizada $g^{(2)}(\tau)$ para dois campos que interagem com átomos de rubídio à temperatura ambiente em condição de EIT. Essa função mede como as flutuações de intensidade estão correlacionadas para instantes de tempo separados por um intervalo $\tau$. Em particular, foram usados campos circularmente polarizados em configuração de $\Lambda$-EIT, como mostra a figura 3.(c). Eles observaram que para tempos $\tau \sim 0$, o meio atômico podia induzir a uma correlação $g^{(2)}(0)>0$ ou anticorrelação $g^{(2)}(0)<0$, dependendo da dessintonia dos campos com relação à transição ao estado excitado. Dentro da janela de transparência $\Delta \omega_{E I T}$, os campos são correlacionados e, consequentemente, as flutuações de intensidade em cada um dos campos mudam simultaneamente e na mesma proporção. Entretanto, quando a dessintonia dos campos se afasta da janela de transparência, os átomos induzem uma anticorrelação entre os campos e, portanto, as flutuações ocorrem na mesma proporção mas em sentido oposto. Assim, os trabalhos liderados por Scully, reportaram a primeira espectroscopia da correlação, limitada porém a um único valor de intensidade dos campos, sem propor uma análise aprofundada da influência dos parâmetros atômicos em tal correlação.

Entretanto, no nosso laboratório (LMCAL) foi adotada uma abordagem no domínio das frequências. Martinelli et al. em [38] apresentaram o segundo trabalho do grupo em que se estuda a transformação RF-RA em um sistema de três níveis em átomos à temperatura ambiente, Eles realizaram a primeira espectroscopia de ruído para observar o mecanismo RF-RA ao medir o ruído de intensidade para diferentes campos com polarizações ortogo- 
nais interagindo com átomos de rubídio à temperatura ambiente em condição transparência induzida, como mostra a figura 4.(a). Em 2007, Luciano Cruz et al. reportaram em [16] o terceiro trabalho feito pelo grupo, onde foram feitas as medidas de correlação de intensidade entre os campos em condição de EIT, junto com um modelo de fase estocástico que leva em conta o mecanismo RF-RA, com átomos à temperatura ambiente. Eles observaram que a correlação de intensidade $C(\omega)$ (que corresponde à transformada de Fourier da função $\left.g^{(2)}(\tau)\right)$ toma valores positivos $(C(\omega)>0)$ para campos fracos, descrevendo assim campos com intensidades correlacionadas. Entretanto, conforme se aumenta a intensidade dos campos, então $C(\omega)<0$ e, portanto, os campos apresentam uma anti-correlação nas suas intensidades. Esta análise da correlação em função das intensidades dos campos, não foi feita por Scully no domínio temporal. Em 2008 Arturo Lezama et al. [39] reportaram um estudo numérico sobre os efeitos quânticos na transmissão do campos interagindo com campos a temperatura ambiente. Eles mostraram que é possível gerar até 30\% de compressão de ruído (squeezing em inglês) na quadratura de fase para amostras altamente densas. No entanto, essa análise numérica não permitia estabelecer uma relação direta entre o squeezing e as variáveis atômicas, para obter um aprofundamento analítico sobre quais os parâmetros físicos que estão por trás dessa propriedade quântica.

Embora fosse claro que, ao dessintonizar os campos e ao aumentar suas intensidades, a correlação podia se transformar em uma anticorrelação nas intensidades dos campos, tanto no domínio do tempo quanto no das frequências, não se aprofundou o entendimento sobre quais as propriedades que mudam nos átomos para eles induzirem esse tipo de correlação ou anti-correlação. Trabalhos como os publicados em [15, 40] estudam a correlação entre os dois campos do sistema $\Lambda$-EIT, mudando a dessintonia de dois fótons, realizando assim uma espectroscopia da função $g^{(2)}(0)$ em átomos à temperatura ambiente. $\mathrm{O}$ espectro da figura 4.(a), feito por Varzhapetyan et al. em [15], mostra que a correlação toma unicamente valores positivos para todas as dessintonias dos campos óticos. Entretanto, a espectroscopia da figura 4.(b), feita por Xiao et al. em [40], mostra que existe uma possível transição de correlação para anticorrelação à medida que aumenta a dessintonia de dois fótons $\delta \in[-10 k H z, 0 k H z]$. Desta forma podemos questionar: quais são as condições do sis- 
tema átomo-luz para que exista correlação ou anticorrelação entre os campos em condição de EIT? O que determina a largura da correlação?, pois ela é uma ordem de magnitude menor do que a largura de transparência $\Delta \omega_{E I T}$. É ela uma largura intrínseca? Era necessário então, fazer um mapeamento dos parâmetros atômicos para dar resposta a tais perguntas.
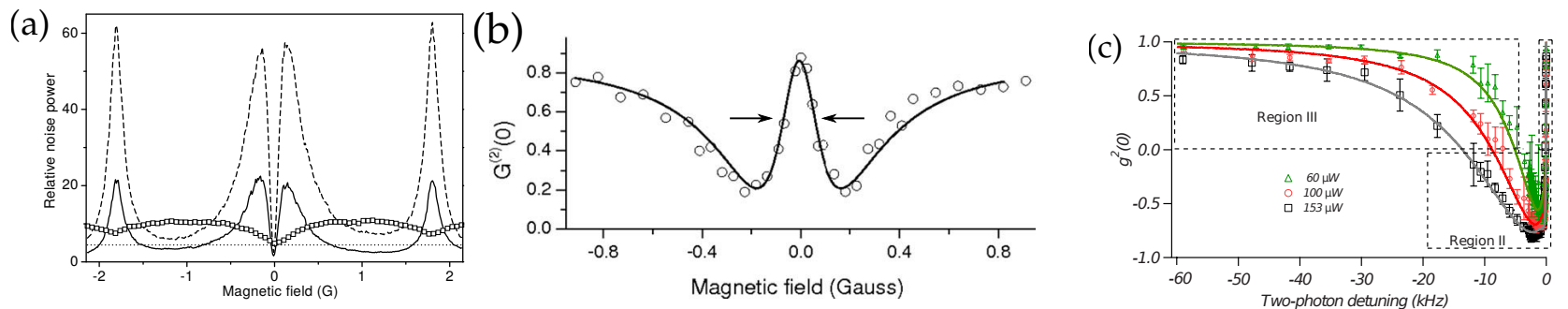

Figura 4: (a) Espectro de ruído dos campos em condição de Hanle-EIT em função do campo magnético, no dominio das frequências [38]. (b) Função de correlação normalizada $g^{(2)}(0)$ em função do campo magnético, medido em [37] e (c) em função da dessintonia de dois fótons medido em [40].

Por outro lado, o fato de realizar as experiências de correlação com átomos à temperatura ambiente, dificulta a sua modelagem teórica, pois é necessário levar em conta o efeito Doppler, integrando a resposta atômica sobre uma distribuição de velocidades. Entre 2007 e 2009, após um árduo trabalho técnico e de preparação instrumental, foi construída a armadilha magneto-ótica (MOT) [41, 42] para resfriamento de átomos de Rubídio no LMCAL (esta não era a primeira versão da MOT no LMCAL). Após a montagem do sistema de captura de imagem para medir a temperatura da nuvem e das primeiras espectroscopias de estados vestidos da nuvem, em 2010 tive a oportunidade de começar a montagem para obter EIT na nuvem de átomos frios. Em 2013 fomos os primeiros a fazer uma ampla análise espectroscópica da correlação em condição de EIT em átomos frios [43, 44], em colaboração com o grupo de física de átomos frios da Universidade Federal de Pernambuco (UFPE). Nesta interface empregamos um laser de diodo para gerar dois feixes que interagem em condição de EIT com uma nuvem de átomos frios numa MOT (ver figura 5). Após a interação com os átomos, as intensidades são medidas por fotodetetores, a partir dos quais podemos medir a correlação de intensidade.

A importância de trabalhar com átomos frios está na direta relação que podemos fazer entre a teoria e o experimento, já que evitamos a integração de velocidades devido ao efeito 
Doppler. No primeiro desses trabalhos na ref.[43], propomos um modelo heurístico para a correlação, em que relacionamos de forma direta a absorção e a dispersão com a função de correlação $g^{(2)}(0)$. Este modelo permitiu aprofundar-nos no estudo das propriedades da espectroscopia de correlação. Junto com este modelo, e com o trabalho feito na ref.[44], mostramos que a espectroscopia de correlação pode ser uma nova técnica para medir a largura intrínseca de EIT, a qual está diretamente relacionada com a taxa de decoerência entre os níveis fundamentais. No entanto, no domínio das frequências, não temos um modelo análogo ao modelo heurístico, que permita fazer tal mapeamento entre $C(\omega)$ e as propriedades de absorção e dispersão atômica.

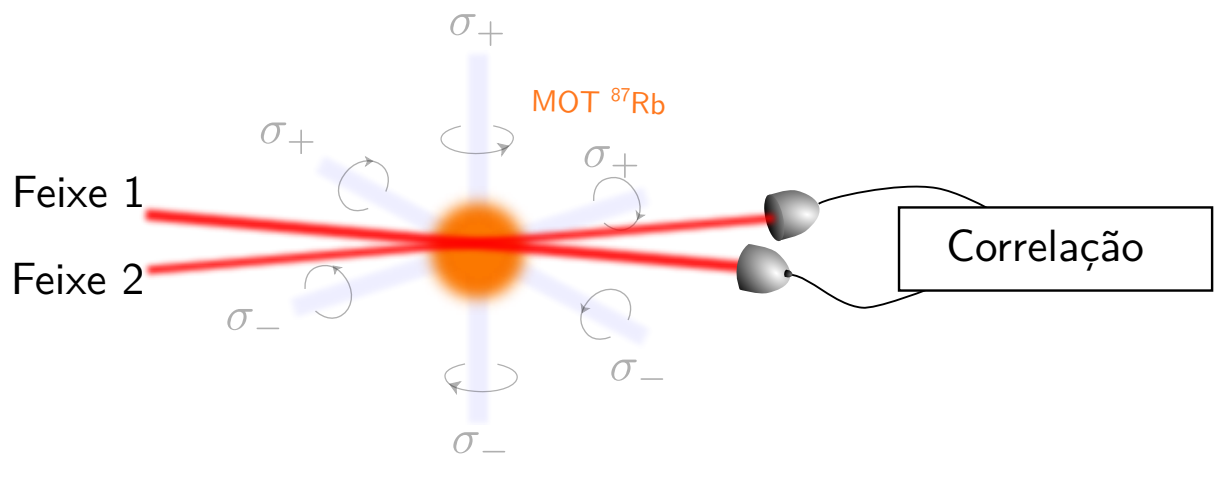

Figura 5: Representação da montagem experimental para medir correlação de intensidade entre dois campos interagindo em condição de EIT com átomos frios.

Neste trabalho apresentarei os principais e mais recentes resultados sobre o estudo de interface de EIT em átomos frios obtidos ao longo deste período do meu doutorado. Um dos objetivos dessa pesquisa foi o estudo teórico e experimental da correlação de intensidade entre dois campos interagindo em condição de EIT com uma amostra de átomos frios, dando continuidade assim às experiências já em andamento no laboratório [44].

Como parte da descrição teórica deste tipo de sistemas, apresentarei um tratamento perturbativo para determinar a correlação de intensidade dos campos induzida pelo meio atômico. Esta proposta, a primeira no domínio das frequências, se mostra compatível com o método de cálculo de Itô empregado nas referências [16, 44], que descrevem satisfatori- 
amente as propriedades da correlação. Mostraremos as principais características do modelo perturbativo, que permite associar diretamente a correlação com as propriedades de absorção e dispersão dos átomos, em contraposição ao modelo de Itô adotado em [16, 44], o qual não permite fazer esse tipo de descrição. Grandes esforços foram feitos para obter uma expressão analítica que permitisse mapear os parâmetros atômicos e a correlação de intensidade no domínio do tempo. No entanto, esse tipo de mapeamento tinha sido proposto unicamente no domínio temporal [43]. O tratamento perturbativo é uma nova ferramenta que nos permite fazer tal mapeamento, e se apresenta como um dos principais resultados da minha pesquisa durante o meu doutorado.

No que diz respeito ao experimento, mostrarei o espectro de correlação para campos dessintonizados até a ordem da largura natural atômica. Mostraremos que em certas condições de dessintonia a correlação pode apresentar ressonâncias nas frequências das bandas laterais, as quais não tinham sido resolvidas até então. Mostraremos que a falta de resolução dessas ressonâncias em trabalhos anteriores $[16,44]$ é devido ao fato da espectroscopia ser feita dentro do intervalo espectral dominado pela emissão espontânea e estimulada. Aliás, mostraremos que elas estão sujeitas a alargamento por potência. Estas propriedades serão descritas de forma satisfatória pelo tratamento perturbativo proposto na primeira parte.

Um segundo aspecto, que estudaremos experimental e teoricamente, é a assimetria espectral da correlação com respeito ao tipo de varredura aplicada na frequência dos campos interagentes. Este é um efeito com o qual nos deparamos no transcurso da investigação das correlações neste tipo de sistema, do qual não temos referência de ter sido observado anteriormente e que exigiu um longo estudo experimental para determinar quais são as causas da aparente "histerese". Demostramos experimental e teoricamente que é graças às forças de espalhamento da luz sobre os átomos que a correlação apresenta tal assimetria espectral.

O texto foi organizado em duas partes. Na primeira, faremos uma revisão teórica da descrição do campo eletromagnético e da sua interação com a matéria. No capítulo 1 faço uma breve revisão da descrição clássica e quântica do campo eletromagnético. Introduziremos o que é a função de correlação e a densidade espectral da luz como os primeiros elementos que permitem caracterizar a luz de forma estatística. Também abordaremos um 
caso especial em que os campos possuem fases cuja dinâmica é de caráter estocástico. Essa descrição estocástica será feita empregando o calculo de Itô. No capítulo 2 faremos uma revisão da polarização linear de um meio atômico. Em seguida, trataremos da interação luz-matéria para um sistema atômico de $N$ níveis. Isto nos permitirá definir de forma compacta o hamiltoniano de interação de campos de luz acoplando um átomo de três níveis, fundamental para a análise feita no capítulo 3. A segunda parte do texto descreve toda a pesquisa relacionada com a espectroscopia de correlação com átomos frios em condição de EIT. No capítulo 3 apresentaremos os aspectos teóricos da correlação em condição de EIT. Começaremos introduzindo o modelo heurístico para a correlação, que descreve de forma simples a relação com as variáveis de absorção e dispersão atômica. Posteriormente revisaremos rapidamente o modelo de Itô usado até então para determinar a correlação de intensidade no domínio das frequências. Na última parte deste capítulo, apresentaremos então os resultados do tratamento perturbativo para calcular a correlação, e discutiremos as suas principais características. No capítulo 4 mostraremos os resultados experimentais da espectroscopia de correlação em regime dessintonizado e em regime dinâmico. Por fim, concluímos este trabalho discutindo os principais resultados e apontaremos algumas das perspectivas para a nossa técnica de espectroscopia de correlação, assim como as da integração dos átomos frios com os outros experimentos que estão em andamento no laboratório. 


\section{Parte I}

\section{Tópicos sobre os campos eletromagnéticos e a sua interação com a matéria}





\section{CAPÍTULO 1}

\section{DESCRIÇÃO DO CAMPO ELETROMAGNÉTICO}

1.1 Descrição clássica do campo eletromagnético . . . . . . . . . . 4

1.2 Propriedades estatísticas da luz ................. 5

1.3 Descrição do campo com flutuações de fase estocástica . . . . . . . . . . . 19

1.4 Tópicos da descrição quântica do campo . . . . . . . . . . . . . . 29

O objetivo deste capítulo é revisar alguns conceitos sobre a descrição do campo eletromagnético, que vão colocar as bases para o estudo dos diferentes fenômenos abordados neste trabalho. Começaremos com a descrição clássica do campo eletromagnético representando como uma onda que se propaga no espaço e que satisfaz as equações de Maxwell. Posteriormente, introduziremos o que é a função de correlação e a densidade espectral da luz como os primeiros elementos que permitem caracterizar a luz de forma estatística. Também abordaremos um caso especial em que os campos possuem fases cuja dinâmica é de caráter estocástico, o que introduzirá conceitos básicos para modelos que estudam a sua interação com meios atômicos, que serão descritos no capítulo 3. No final do capítulo faremos uma breve revisão da descrição quântica do campo para analisar o estado coerente da luz. Esse estado em particular descreve o que seria a luz emitida por um laser, o qual representa uma das principais ferramentas nas áreas de física atômica e ótica quântica. 


\subsection{Descrição clássica do campo eletromagnético}

$\mathrm{Na}$ ausência de fontes de carga elétrica, a eletrodinâmica mostra que os campos eletromagnéticos são regidos pela equação de propagação

$$
\begin{aligned}
\nabla^{2} \mathbf{E}-\frac{1}{c^{2}} \frac{\partial^{2} \mathbf{E}}{\partial t^{2}} & =0, \\
\nabla^{2} \mathbf{B}-\frac{1}{c^{2}} \frac{\partial^{2} \mathbf{B}}{\partial t^{2}} & =0 .
\end{aligned}
$$

A solução para estas equações são soluções de ondas propagantes no tempo e no espaço. Em particular para o campo elétrico $\mathbf{E}$, temos uma onda elétrica se propagando em direção $\mathrm{k}$ e com frequência $\omega$, tal que

$$
\mathbf{E}(\mathbf{r}, t)=\frac{1}{2}\left[\mathcal{E} e^{i(\mathbf{k} \cdot \mathbf{r}-\omega t)} \hat{\mathbf{e}}(\theta, \epsilon)+\mathcal{E}^{*} e^{-i(\mathbf{k} \cdot \mathbf{r}-\omega t)} \hat{\mathbf{e}}^{*}(\theta, \epsilon)\right]
$$

em que $\mathcal{E}$ representa a amplitude (complexa em geral) e $\hat{\mathbf{e}}(\theta, \epsilon)$ o vetor de polarização do campo, que satisfaz $\mathbf{k} \cdot \hat{\mathbf{e}}(\theta, \epsilon)=0$, onde $\theta$ e $\epsilon$ representam o ângulo de polarização e a elipticidade, respectivamente $[45,46]$. Dado o caráter complexo do campo, podemos definir a amplitude do campo como $\mathcal{E}=|\mathcal{E}| e^{i \phi}=\left(E_{P}+i E_{Q}\right)$, de modo que ele seja descrito por

$$
\mathbf{E}(\mathbf{r}, t)=\left[E_{P} \cos (\mathbf{k} \cdot \mathbf{r}-\omega t)+E_{Q} \sin (\mathbf{k} \cdot \mathbf{r}-\omega t)\right] \hat{\mathbf{e}}(\theta, \epsilon),
$$

onde $E_{P}$ e $E_{Q}$ correspondem às quadraturas do campo [7]. A figura 1.1.a mostra na representação de Fresnel (diagrama de fasor) a amplitude do campo elétrico representando $\mathcal{E}$ como um vetor de amplitude $|\mathcal{E}|$ e fase $\phi$, cujas componentes reais e imaginárias correspondem às quadraturas do campo. A figura 1.1.b mostra a evolução temporal da onda na quadratura $E_{P}$ com fase $\varphi(t)=\mathbf{k} \cdot \mathbf{r}_{o}-\omega t$.

De forma geral, os campos eletromagnéticos podem ser representados como a superposição 
(a)

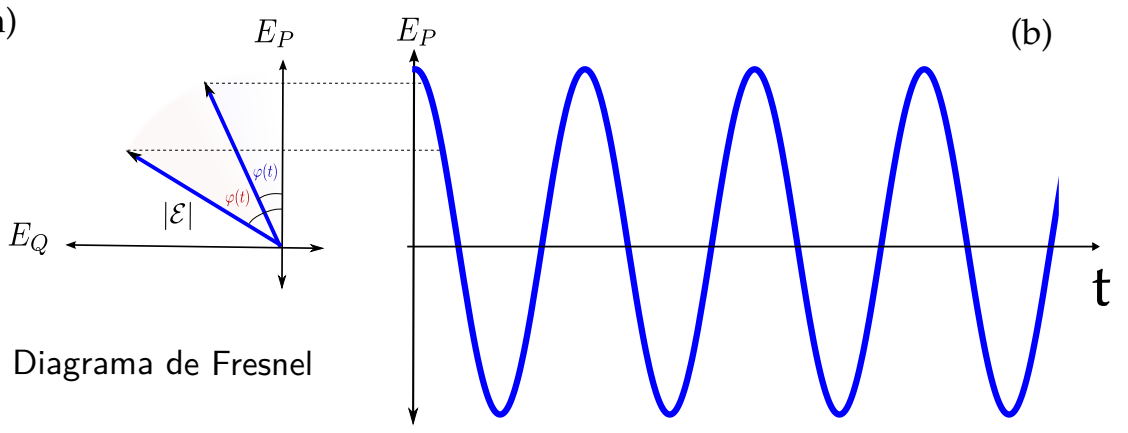

Figura 1.1: (a) Representação de fresnel da amplitude $\mathcal{E}$ em termos das suas quadraturas $E_{p}$ e $E_{Q}$. (b) Representação da evolução temporal do campo elétrico $\mathbf{E}(\mathbf{r}, t)$ mediante diagrama de fasores. Ele é descrito como um vetor com amplitude $|\mathcal{E}|$ e fase $\varphi(t)$.

de campos com modos de frequência ou polarização diferentes, tal que:

$$
\begin{aligned}
& \mathbf{E}(\mathbf{r}, t)=\sum_{n, l}\left(\mathcal{E}_{n, l} e^{i\left(\mathbf{k}_{n} \cdot \mathbf{r}-\omega_{n} t\right)} \hat{\mathbf{e}}_{n, l}-\mathcal{E}_{n, l}^{*} e^{-\left(i \mathbf{k}_{n} \cdot \mathbf{r}-\omega_{n} t\right)} \hat{\mathbf{e}}_{n, l}^{*}\right) \\
& \mathbf{B}(\mathbf{r}, t)=\frac{1}{c} \sum_{n, l}\left(\mathcal{E}_{n, l} e^{i\left(\mathbf{k}_{n} \cdot \mathbf{r}-\omega_{n} t\right)} \hat{\mathbf{k}}_{n} \times \hat{\mathbf{e}}_{n, l}-\mathcal{E}_{n, l}^{*} e^{-\left(i \mathbf{k}_{n} \cdot \mathbf{r}-\omega_{n} t\right)} \hat{\mathbf{k}}_{n} \times \hat{\mathbf{e}}_{n, l}^{*}\right)
\end{aligned}
$$

onde $n$ determina modos de frequência e $l$ determina modos de polarização ${ }^{1}$ associados $^{2}$ ao modo $n$. Notemos que a onda plana monocromática é um caso particular esta solução. Agora, quando às amplitudes $\mathcal{E}_{n, l}$ seguem uma distribuição gaussiana em frequências, e existe uma diferença de fase bem definida $\Delta=\phi_{n}-\phi_{n^{\prime}}$ sendo $\phi_{n}=\mathbf{k}_{n} \cdot \mathbf{r}-\omega_{n} t$, as eqs.(1.4a) descrevem a luz emitida por um laser que se propaga em forma de pulso. No entanto, se a diferença de fase é aleatória ou incoerente, temos outro tipo de fonte de luz, para as quais é mais conveniente o estudo das suas propriedades estatísticas.

\subsection{Propriedades estatísticas da luz}

Até este ponto, temos considerado a descrição clássica de uma onda eletromagnética que oscila com frequência, amplitude e fase bem definida. No entanto esta concepção da luz descreve idealmente o que podemos encontrar na natureza. Tomemos, por exemplo, a luz

\footnotetext{
${ }^{1}$ A polarização $\hat{\mathbf{e}}_{n, l}$ em geral pode ser complexa. Exemplos disso são as polarizações circulares $\sigma_{+}$e $\left.\sigma_{-}\right\}$.
} 
emitida por um conjunto de átomos de forma independente num intervalo de tempo finito muito mais curto que o tempo de medida. É possível então observar flutuações na intensidade da luz em torno de um valor médio [47]. Além disso, se levamos em conta que os átomos podem estar em movimento, isto induzirá deslocamentos na frequência emitida, alargando então a sua distribuição espectral. Portanto, as flutuações de intensidade e o alargamento espectral podem ser manifestações das mesmas propriedades radiativas dos átomos que constituem as fontes de luz [48].

Algumas fontes de luz, como a lâmpada de filamento ou o sol são exemplos de fontes caóticas, onde as velocidade atômicas e as colisões aleatórias alteram a distribuição espectral e induzem flutuações de intensidade. Um outro tipo de luz é o laser, cujas propriedades estatísticas são diferentes do caso de fontes de luz caótica. A luz emitida por um laser satisfaz a condição de mínima incerteza nas flutuações de amplitude e intensidade, para as quais são necessárias uma descrição quântica como será revisado na seção 1.9.

Nesta seção abordaremos os principais elementos empregados na descrição das propriedades estatísticas da luz, como a função de correlação de intensidade e a densidade espectral de um campo eletromagnético. A abordagem com que faço a revisão destes conceitos é baseada principalmente na ref.[48].

\subsubsection{Função de correlação de primeira ordem}

A luz emitida por uma lâmpada pode ser descrita como a de um conjunto de átomos emitindo luz com a mesma frequência $\omega_{0}$. No entanto, os átomos estão sujeitos a colisões (elásticas) que alteram aleatoriamente a fase $\phi(t)$ da luz emitida, o que as caracteriza como fontes de luz caóticas. Portanto, se consideramos unicamente a parte temporal, cada um deles emite uma onda $E_{i}(t)=\mathcal{E}_{i} \exp \left\{-i \omega_{0} t+i \phi_{i}(t)\right\}$ com a mesma polarização e, portanto, o campo resultante será

$$
\begin{aligned}
E(t) & =E_{1}(t)+E_{2}(t)+\cdots+E_{N}(t) \\
& =E_{0} e^{-i \omega_{0} t}\left[e^{i \phi_{1}(t)}+e^{i \phi_{2}(t)}+\cdots+e^{i \phi_{N}(t)}\right]
\end{aligned}
$$


onde consideramos que todos os átomos emitem com a mesma amplitude $\mathcal{E}_{i}=E_{0}$. Esta superposição pode ser claramente representada por meio de um diagrama de fasores como mostra a figura 1.2.(a). Nela o campo resultante pode ser expressado como $E(t)=E_{0} \alpha(t)$ $\times \exp \left\{-i \omega_{0} t+\varphi(t)\right\}$ onde $\alpha(t)$ e $\varphi(t)$ representam a amplitude e a fase do campo resultante, respectivamente. Portanto a intensidade do campo é $I(t)=E_{0}^{2} \alpha(t)^{2}$.
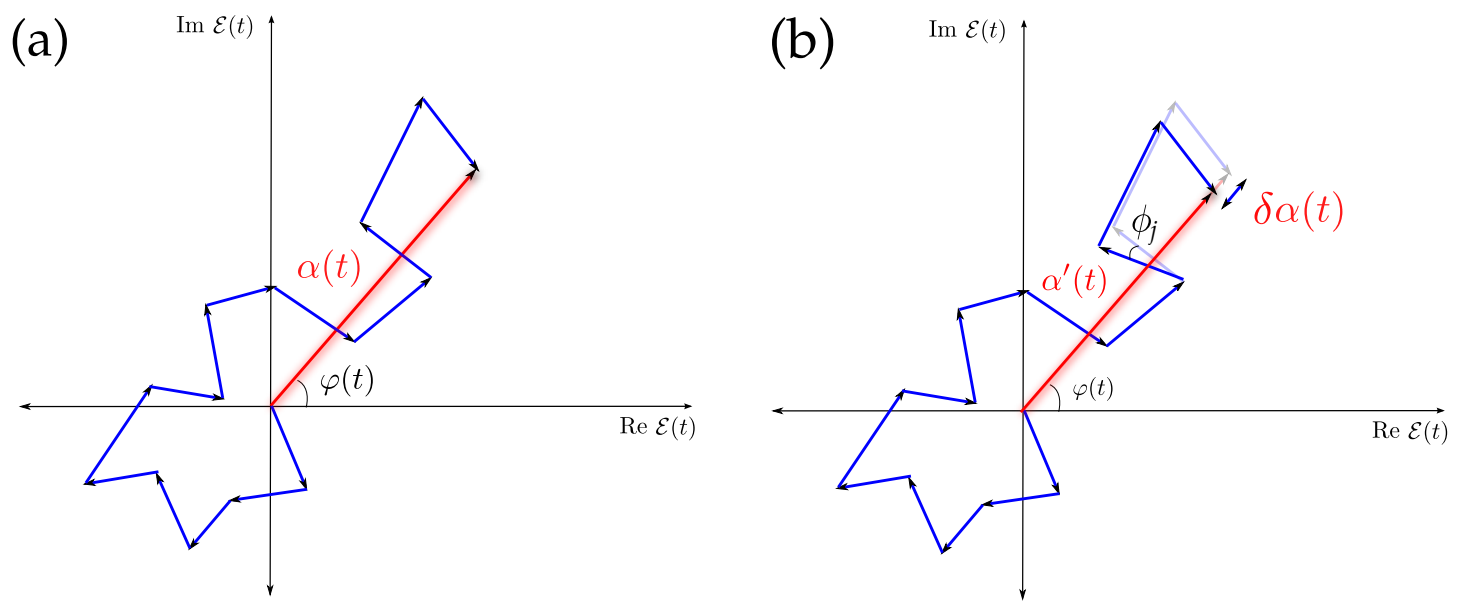

Figura 1.2: (a) Representação de fresnel da luz emitida por um campo caótico, onde o campo resulte tem uma amplitude $\alpha(t)$ e uma fase $\varphi(t)$. (b) A flutuação de fase $\phi_{i}$ da partícula i-ésima, gera uma flutuação na amplitude, se tornado $\alpha^{\prime}(t)=\alpha(t)-\delta \alpha(t)$.

A amplitude e a fase do campo vão apresentar flutuações aleatórias conforme as fases de cada fonte $\phi_{i}(t)$ flutuem, como mostra a figura 1.2.(b). Isto vai se traduzir em flutuações na intensidade $I(t)$, já que $I(t) \rightarrow I^{\prime}(t)=E_{0}^{2} \alpha^{\prime}(t)^{2}$. Neste exemplo simples está um dos principais efeitos que estudamos neste trabalho: as flutuações de intensidade induzidas por flutuações de fase. Em particular, veremos como a luz, com certas características, ao se propagar por um meio atômico, vai apresentar essa transformação de flutuações de fase para ruído de intensidade.

Uma das principais definições que caracterizam uma determinada fonte de luz é o tempo de coerência. Ele se caracteriza por ser o intervalo de tempo $\tau_{c}$ em que a flutuação de intensidade do campo é limitada. Dado este tempo de coerência, podemos então associar um comprimento de coerência que é dado por $\lambda_{c}=c \tau_{c}$. Este comprimento de coerência é maior do que o comprimento de onda da luz emitida. Como podemos medir essa coerência? A 
função de correlação de primeira ordem pode nos dar a resposta. Para intervalos de tempo menores que $\tau_{c}$ o campo $E(t)$ não flutua e, portanto, ele mantêm alguma correlação. Porém, se comparamos o campo para instantes de tempo separados por um intervalo muito maior do que $\tau_{c}$, o campo apresentara várias flutuações e, portanto, o campo não é correlacionado. Esta correlação é quantificada pela função de primeira ordem

$$
g^{(1)}(\tau)=\frac{\left\langle E^{*}(t) E(t+\tau)\right\rangle}{\left\langle E^{*}(t) E(t)\right\rangle}
$$

Para o caso de uma onda ideal estável como a da eq.(1.2), a função de correlação é $\left|g^{(1)}(\tau)\right|=$ 1, ou seja, o campo está correlacionado para qualquer intervalo de tempo $\tau$. Para o caso de luz caótica da eq.(1.5) as fases $\phi_{i}(t)$ flutuam de forma independente, de modo que $\left\langle E^{*}(t) E(t+\right.$ $\tau)\rangle=N E_{0}^{2} \exp \left\{-i \omega_{0} \tau-\tau / \tau_{c}\right\}$, e assim, a função de correlação de primeira ordem é

$$
g^{(1)}(\tau)=e^{-i \omega_{0} \tau-|\tau| / \tau_{c}}
$$

o que define um perfil Lorentziano, tal que para $|\tau|>>\tau_{c}$ a função $\left|g^{(1)}(\tau)\right| \rightarrow 0$. O perfil da correlação muda, quando consideramos que o conjunto de átomos que emitem luz está em constante movimento, de modo que geram um deslocamento em frequência. Portanto, o tipo de luz emitida por essa fonte vai apresentar um alargamento por efeito Doppler e a sua função de correlação é dada por

$$
g^{(1)}(\tau)=e^{-i \omega_{0} \tau-\delta^{2} \tau^{2}}
$$

onde o seu tempo de coerência é $\tau \approx 1 / \delta$. Neste caso temos uma função de correlação Gaussiana. A figura 1.3 mostra a função de correlação para estes três tipos de fontes de luz: estável, caótica por colisões e caótica com deslocamento Doppler.

Manifestações da função de primeira ordem estão presentes nas experiencias de interferência, como acontece no experimento de duas fendas de Young. Nela se faz passar a luz emitida por uma fonte caótica (uma lâmpada) por duas fendas. A luz que passa pelas fen- 


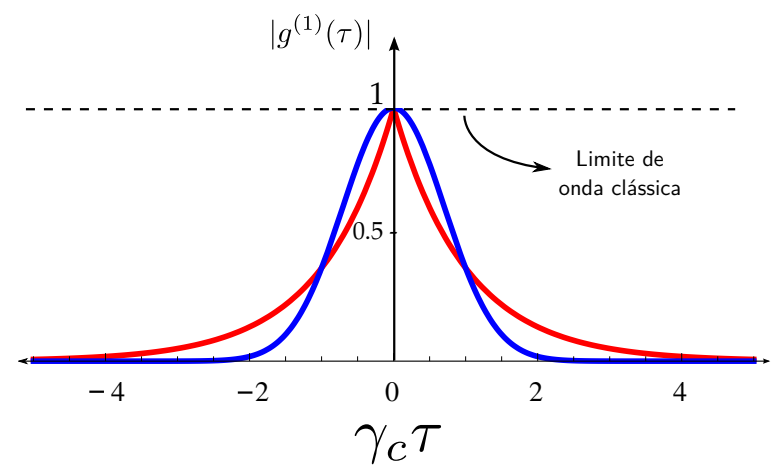

Figura 1.3: Função de correlação $\left|g^{(1)}(\tau)\right|$ em função do intervalo de tempo $\tau$. Em vermelho temos a função de correlação para uma fonte caótica e em azul para uma fonte caótica com alargamento por efeito Doppler. A linha tracejada mostra o limite dado por uma onda estável.

das se propaga em direção a uma tela na qual os campos se superpõem. Em um ponto $\mathbf{r}$ da segunda tela o campo é tal que $\mathbf{E}(\mathbf{r}, t)=\mathbf{E}\left(\mathbf{r}, t_{1}\right)+\mathbf{E}\left(\mathbf{r}, t_{2}\right)$, onde os tempos de atraso são $t_{i}=t-\mathbf{r}_{i} / c$. A intensidade media $\langle I\rangle=k\left\langle|\mathbf{E}(\mathbf{r}, t)|^{2}\right\rangle$ no ponto $\mathbf{r}$ na tela é dado por

$$
\begin{aligned}
\langle I(t)\rangle & =\kappa\left\langle|\mathbf{E}(\mathbf{r}, t)|^{2}\right\rangle=\frac{\kappa}{t_{d}} \int_{0}^{t_{d}} d t^{\prime}\left|\mathbf{E}_{d}\left(\mathbf{r}, t^{\prime}\right)\right|^{2} \\
& =\kappa\left[\left\langle\left|\mathbf{E}\left(\mathbf{r}, t_{1}\right)\right|^{2}\right\rangle+\left\langle\left|\mathbf{E}\left(\mathbf{r}, t_{2}\right)\right|^{2}\right\rangle+2 \operatorname{Re}\left\{\left\langle\mathbf{E}^{*}\left(\mathbf{r}, t_{1}\right) \cdot \mathbf{E}\left(\mathbf{r}, t_{2}\right)\right\}\right\rangle\right]
\end{aligned}
$$

Dado que a defasagem espacial se reflete em um atraso da luz no tempo, então os campos podem ser expressados como $\mathbf{E}\left(\mathbf{r}, t_{i}\right)=\mathbf{E}\left(t-s_{i} / c\right)$, onde $s_{i}=\left|\mathbf{r}_{i}\right|$. Desta forma temos então que cada campo toma a mesma forma que os campos da eq.(1.5) e, portanto, os valores médios $\left\langle E^{*}\left(t_{1}\right) E\left(t_{2}\right)\right\rangle=N E_{0}^{2} \exp \left\{-i \omega \tau-\tau / \tau_{c}\right\}$ onde $\tau=\left|\mathbf{r}_{1}-\mathbf{r}_{2}\right| / c$. Isto leva a um padrão de interferência na intensidade da luz descrito por

$$
\langle I(t)\rangle=2 \kappa N E_{0}^{2}\left[1+\exp \left\{-|\tau| / \tau_{c}\right\} \cos \left(\omega_{0} \tau\right)\right]
$$

onde o termo de interferência $\cos \left(\omega_{0} \tau\right)$ é atenuado pelo fator de coerência $\exp \left\{-|\tau| / \tau_{c}\right\}$. A visibilidade quantifica o contraste com o qual se observam as franjas de interferência e muda 
segundo o tipo de fonte de luz que se emprega neste tipo de experiência. Neste caso ela é

$$
V=\frac{\langle I(t)\rangle_{\max }-\langle I(t)\rangle_{\min }}{\langle I(t)\rangle_{\max }+\langle I(t)\rangle_{\min }}=\exp \left\{-|\tau| / \tau_{c}\right\}
$$

Isto mostra que para o caso em que empregamos uma fonte estável ideal, ou seja $\tau_{c} \rightarrow \infty$, a visibilidade é $V=1$. No entanto, o que mostra a expressão (1.12) é que para fontes caóticas a visibilidade nunca poderia ser máxima.

Um exemplo histórico da aplicação dessa técnica interferométrica para descrever a coerência de um sistema físico é o do interferômetro de Michelson et.al em 1920 [47, 49]. Eles propuseram medir o tamanho de uma estrela mediante interferometria. As estrelas não são fontes de emissão pontuais, são na realidade um conjunto de fontes pontuais que emitem luz dentro de um volume determinado. Para fazer este experimento eles exploraram a propriedade de coerência espacial da luz, ou seja, exploraram o fato da amplitude e a fase das ondas eletromagnéticas emitidas por fontes próximas possuírem uma correlação que possibilita a interferências entre elas. A luz emitida por fontes remotas tem amplitudes e fases muito diferentes, que impossibilitam a interferência entre as ondas. Já para fontes de luz próximas, as ondas emitidas tem amplitudes e fases certas que possibilitam a interferência. Portanto, eles aplicaram esse conceito ao tamanho de uma estrela. Somente quando a luz for emitida da mesma região da estrela é possível registrar um padrão de interferência no interferômetro mostrado na figura 1.4.(a).

Contudo, a montagem proposta por Michelson, carregava várias complicações técnicas devido às flutuações atmosféricas e instrumentais. Pois, a correlação de primeira ordem é sensível à fase, como podemos observar nas eqs.(1.7) e (1.8). Quando a luz se propaga pela atmosfera, a correlação $g^{(1)}(\tau)$ adquiri uma fase $\omega_{0} \tau \rightarrow \omega_{0} \tau+\phi$ onde $\phi$ é aleatória. Isto atenua as franjas de interferência e dificulta a medida mediante o interferômetro de Michelson. Uma outra técnica foi proposta por R. Hanbury Brown e R.Q. Twiss [50], em 1957, usando uma lâmpada de mercúrio como fonte de luz e posteriormente aplicada à medida de tamanho das estrelas em 1959. Eles propuseram um interferômetro baseado na medida de intensidade da luz mediante detetores fotomultiplicadores para medir o tamanho de uma 


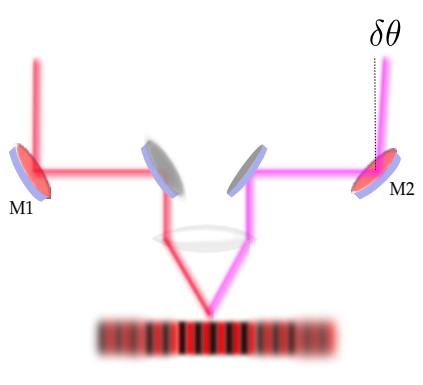

(a)

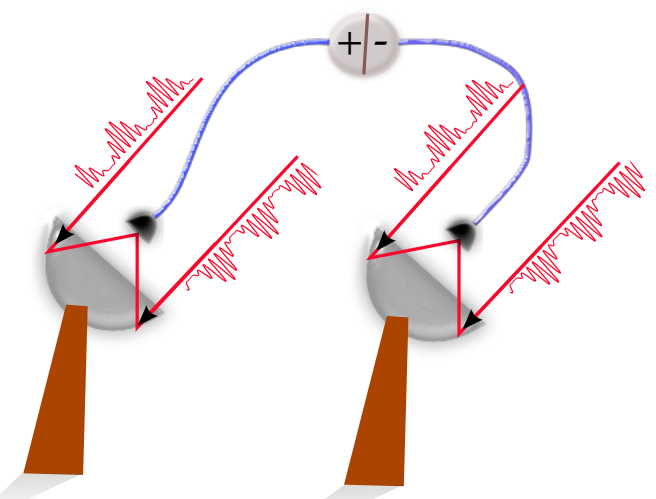

(b)

Figura 1.4: (a) Interferômetro de Michelson. (b) Interferômetro de intensidade de R. Hanbury Brown e R. Twiss.

estrela como mostra a figura 1.4.(b). Este interferômetro explora a ideia de flutuações de intensidade presentes na luz emitida pelas estrelas. Especificamente, se propuseram a medir a correlação de flutuações de intensidade entre diferentes regiões da estrela. Isto é, para duas fontes próximas com uma distância máxima de um comprimento de coerência, a luz interfere e as flutuações de intensidade são correlacionadas. Porém, se elas se encontrarem à distância além do comprimento de coerência, as ondas não interferem e a correlação de intensidade é nula.

O inovador na experiencia de Hanbury Brown e Twiss, é que além de medir a fotocorrente em cada detector, eles misturaram os sinais das fotocorrentes, ou seja, mediram o valor médio de $\langle I(t) I(t+\tau)\rangle$, que define não uma medida de correlação de primeira ordem nos campos, mas sim, uma medida de correlação de segunda ordem, já que $\langle I(t) I(t+\tau)\rangle=$ $\left.\left\langle E^{*}(t) E^{*}(t+\tau) E^{(} t\right) E^{*}(t)\right\rangle$. Assim eles mediram um grau de coerência superior com relação ao experimento de Michelson. Mais adiante, veremos que esta correlação de segunda ordem não depende da fase do campo e, portanto, é insensível a defasagens induzidas pela atmosfera. 


\subsubsection{Função de correlação $g^{(2)}(\tau)$}

A partir da correlação de intensidades, pode-se definir o grau de coerência temporal de segunda ordem como

$$
g^{(2)}(\tau)=\frac{\langle I(t) I(t+\tau)\rangle}{\langle I(t)\rangle\langle I(t+\tau)\rangle}=\frac{\left\langle E^{*}(t) E^{*}(t+\tau) E(t) E(t+\tau)\right\rangle}{\left\langle E^{*}(t) E(t)\right\rangle^{2}}
$$

ou também chamada função de correlação normalizada. Para um campo puramente clássico em que não consideramos flutuações na amplitude do campo, ou seja $E(t)=E_{0}$, a função de correlação é dada por

$$
g^{(2)}(\tau)=\frac{\left\langle E_{0}^{4}\right\rangle}{\left\langle E_{0}^{2}\right\rangle^{2}}=1
$$

Agora, para um campo que possui flutuações de intensidades clássicas, a função de correlação toma valores $\infty \geq g^{(2)}(0) \geq 1$. Por exemplo, a função de correlação normalizada para uma fonte caótica e uma fonte com distribuição Gaussiana é dada por

$$
\begin{array}{ll}
g^{(2)}(\tau)=1+e^{-2 \tau / \tau_{c}} & \text { Fonte caótica. } \\
g^{(2)}(\tau)=1+e^{-\delta^{2} \tau^{2}} & \text { Fonte com Distr. Gaussiana. }
\end{array}
$$

Pode se mostrar que para este tipo de fonte a função de correlação pode ser escrita como $g^{(2)}(\tau)=1+\left|g^{(1)}(\tau)\right|^{2}$, de acordo com as eqs.(1.7) e (1.8). Notemos então, que elas não dependem da fase dos campos, o que caracteriza o interferômetro de Hanbury Brown \& Twiss empregado para medir o tamanho de estrelas em 1959, pois ele é insensível a alterações induzidas pela atmosfera.

Para entender a relação entre a função de correlação e as flutuações da intensidade, consideremos a intensidade da forma $I(t)=\bar{I}+\delta I(t)$ tal que $\bar{I}$ é o valor médio e $\delta I(t)$ representa a flutuação de intensidade com média nula. A variância, que quantifica o deslocamento quadrático médio da flutuação $\delta I$, é definida como

$$
\operatorname{Var}\{I(t)\}=\Delta^{2} I(t)=\left\langle\delta I^{2}(t)\right\rangle=\left\langle I(t)^{2}\right\rangle-\bar{I}^{2}
$$


onde relacionamos a variância com o valor médio $\langle I(t)\rangle$ e o segundo momento da intensidade $\left\langle I(t)^{2}\right\rangle$. Portanto, a função de correlação é

$$
\begin{aligned}
g^{(2)}(\tau) & =\frac{\langle(\bar{I}+\delta I(t))(\bar{I}+\delta I(t+\tau))\rangle}{\bar{I}^{2}} \\
& =1+\frac{\langle\delta I(t) \delta I(t+\tau)\rangle}{\bar{I}^{2}}
\end{aligned}
$$

de modo que para $\tau=0$, pela variância da eq.(1.17) a função de correlação é $g^{(2)}(0)=$ $1+\frac{\Delta^{2} I(t)}{\bar{I}^{2}}$.

Desta forma, relacionamos a função de correlação de segunda ordem diretamente com a variância da intensidade dos campos, quando consideramos a defasagem temporal $\tau=0$. Esta relação mostra explicitamente que ao considerarmos campos que apresentam flutuações na intensidade devido a fontes clássicas, ou seja, $\Delta^{2} I>0$, a função de correlação será sempre $g^{(2)}(0)>1$. Para medir este tipo de correlação, Hanbury Brown \& Twiss passaram a luz emitida pela lâmpada de mercúrio por um divisor de feixes, e a intensidade de cada um dos campos foi medida por um fotomultiplicador, como mostra figura 1.5. Eles mediram a correlação da eq.(1.18) tal que $g^{(2)}(\tau)=1+\left\langle\delta I\left(t_{1}\right) \delta I\left(t_{2}\right)\right\rangle / \bar{I}^{2}$ sendo $\tau=t_{2}-t_{1}$.

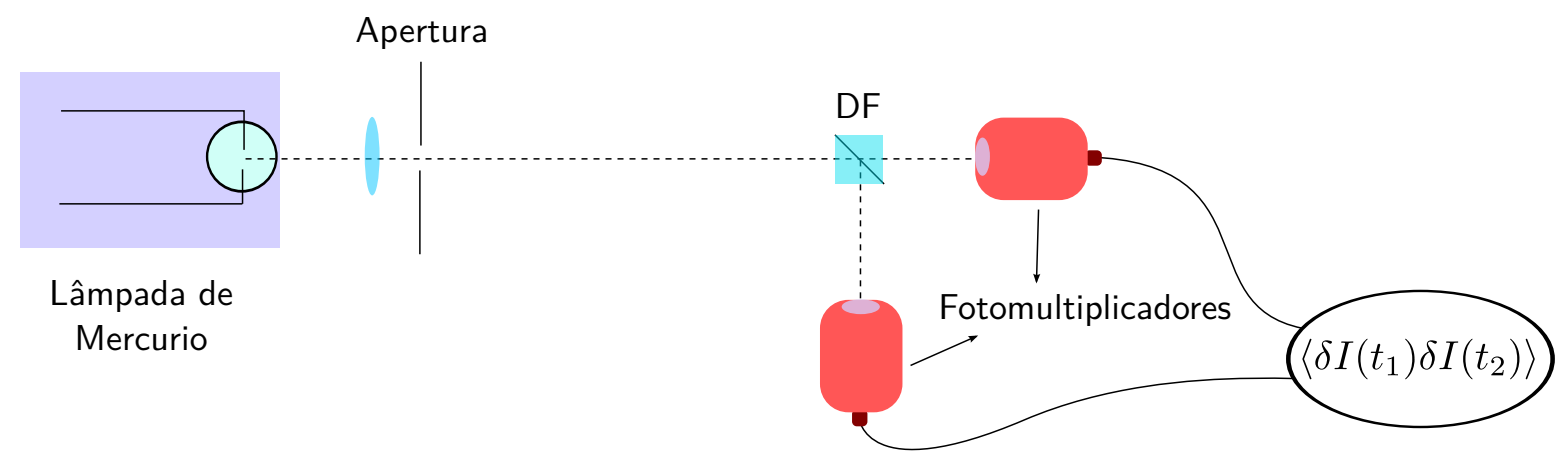

Figura 1.5: Arranjo experimental para medir correlação de segunda ordem proposto por R. Hanbury Brown e R. Twiss.

Temos definida então a função de correlação normalizada de segunda ordem para um campo eletromagnético que mede a probabilidade de detectar flutuações de um mesmo campo de luz num intervalo de tempo $\tau$. Entretanto, quando consideramos dois ou mais campos de fontes diferentes, por exemplo $\mathbf{E}_{1}(\mathbf{r}, t)$ e $\mathbf{E}_{2}(\mathbf{r}, t)$, definimos a correlação cruzada 
como a média de um produto cruzado de intensidades, tal que

$$
g_{12}^{(2)}(\tau)=\frac{\left\langle I_{1}(t) I_{2}(t+\tau)\right\rangle}{\left\langle I_{1}(t)\right\rangle\left\langle I_{2}(t+\tau)\right\rangle}
$$

Em um sistema bipartite é conveniente definir propriedades que caracterizem o sistema como um todo. Uma delas é a desigualdade de Cauchy-Schwarz definida como

$$
g_{12}^{(2)}(\tau) \leq \sqrt{g_{11}^{(2)}(0) g_{22}^{(2)}(0)}
$$

que relaciona a função de correlação cruzada de segunda ordem $g_{12}^{(2)}(\tau)$ com a função de correlação de cada campo $g_{11}^{(2)}(0)$ e $g_{22}^{(2)}(0)$ dadas pela definição (3.5). Podemos então redefinir a correlação de intensidade entre os campos a partir da desigualdade de Cauchy-Schwarz como

$$
G_{12}^{(2)}(\tau)=\frac{g_{12}^{(2)}(\tau)}{\sqrt{g_{11}^{(2)}(0) g_{22}^{(2)}(0)}}=\frac{\left\langle I_{1}(t) I_{2}(t+\tau)\right\rangle}{\sqrt{\left\langle I_{1}^{2}(t)\right\rangle\left\langle I_{2}^{2}(t)\right\rangle}}
$$

e consequentemente limitada por $\left|G^{(2)}(\tau)\right| \leq 1$. De acordo com a referência [51], esta correlação pode se reescrever em termos das flutuações dos campos tal que

$$
\tilde{g}_{12}^{(2)}(\tau)=\frac{\left\langle\delta I_{1}(t) \delta I_{2}(t+\tau)\right\rangle}{\sqrt{\Delta^{2} I_{1}(t) \Delta^{2} I_{2}(t)}}=\frac{\left\langle\delta I_{1}(t) \delta I_{2}(t+\tau)\right\rangle}{\sqrt{\Delta^{2} I_{1}(t) \Delta^{2} I_{2}(t)}}
$$

que também é limitada por $0 \leq\left|\tilde{g}^{(2)}(\tau)\right| \leq 1$.

No capítulo 3 estudamos esta função de correlação cruzada de segunda ordem quando dois campos interagem com um meio atômico, em condição de transparência eletromagneticamente induzida.

\subsubsection{Densidade espectral do campo $S(\omega)$}

Viu-se então como as flutuações de intensidade da luz são propriedades das fontes de luz e que podem ser caracterizadas pela função de correlação de segunda ordem. Agora, se considerarmos que as fontes dos campos estão sujeitas a um constante movimento, elas 
não somente geram ondas com diferentes amplitudes, mas também emitem luz com certa distribuição espectral, devido ao efeito Doppler. Consequentemente, temos flutuações de intensidade com diferentes componentes espectrais. Portanto, é necessário completar a descrição dos campos feita até agora com uma distribuição espectral, diferente de uma função delta, como seria para o caso ideal clássico.

Para ilustrar como as flutuações podem gerar alargamentos espectrais, podemos analisar o caso da fonte caótica e o da distribuição Doppler. Para começar, a forma geral da distribuição espectral do campo $\mathcal{E}(t)$ é dada pela sua transformada de Fourier

$$
\mathcal{E}(\omega)=\frac{1}{2 \pi} \int_{-\infty}^{\infty} \mathcal{E}(t) e^{i \omega t} d t
$$

Portanto, a intensidade média do campo com frequência $\omega$ é proporcional a

$$
|\mathcal{E}(\omega)|^{2}=\frac{1}{4 \pi^{2}} \int_{-\infty}^{\infty} \int_{-\infty}^{\infty} \mathcal{E}^{*}(t) \mathcal{E}(t+\tau) e^{i \omega \tau} d t d \tau
$$

Se as flutuações que apresenta o campo $E(t)$ possuem um caráter estacionário, ou seja, elas permanecem iguais para tempos $t \rightarrow \infty$, então

$$
\left\langle\mathcal{E}^{*}(t) \mathcal{E}(t+\tau)\right\rangle=\frac{1}{T} \int_{-\infty}^{\infty} \mathcal{E}^{*}(t) \mathcal{E}(t+\tau) d t
$$

em que $T$ representa um intervalo de tempo grande suficiente (mas finito) no qual as flutuações do campo acontecem. Assim, o espectro da intensidade média do campo da eq.(1.24) é reescrito como

$$
|\mathcal{E}(\omega)|^{2}=\frac{T}{4 \pi^{2}} \int_{-\infty}^{\infty}\left\langle\mathcal{E}^{*}(t) \mathcal{E}(t+\tau)\right\rangle e^{i \omega \tau} d \tau
$$

Ao normalizar pela soma de todas as componentes espectrais $\int|\mathcal{E}(\omega)|^{2} d \omega=(T / 2 \pi)\left\langle\mathcal{E}^{*}(t) \mathcal{E}(t)\right\rangle$, 
temos então a distribuição espectral do campo

$$
\begin{aligned}
\sigma(\omega) & =\frac{|\mathcal{E}(\omega)|^{2}}{\int|\mathcal{E}(\omega)|^{2} d \omega}=\frac{\int_{-\infty}^{\infty}\left\langle\mathcal{E}^{*}(t) \mathcal{E}(t+\tau)\right\rangle e^{i \omega \tau} d \tau}{\left\langle\mathcal{E}^{*}(t) \mathcal{E}(t)\right\rangle} \\
& =\int_{-\infty}^{\infty} g^{(1)}(\tau) e^{i \omega \tau} d \tau
\end{aligned}
$$

Este resultado descreve a relação entre a distribuição espectral $\sigma(\omega)$ e a função temporal de correlação de primeira ordem. Se consideramos então a função de correlação a primeira ordem de uma fonte com a distribuição Gaussiana descrita pela eq.(1.16), obtemos uma distribuição espectral Gaussiana

$$
\begin{aligned}
\sigma(\omega) & \left.=\int_{-\infty}^{\infty} \exp \left\{-i \omega_{0} \tau-\delta^{2} \tau^{2}\right\}\right\rangle e^{i \omega \tau} d \tau \\
& =\frac{1}{\pi \delta^{2}} e^{-\frac{\left(\omega-\omega_{0}\right)^{2}}{4 \delta^{2}}}
\end{aligned}
$$

e para uma fonte caótica, uma distribuição Lorentziana

$$
\begin{aligned}
\sigma(\omega) & \left.=\int_{-\infty}^{\infty} \exp \left\{-i \omega_{0} \tau-|\tau| / \tau_{c}\right\}\right\rangle e^{i \omega \tau} d \tau \\
& =\frac{\tau_{c}}{\pi} \frac{1}{\left(\omega-\omega_{0}\right)^{2}+\left(1 / \tau_{c}\right)^{2}}
\end{aligned}
$$

onde lembramos que $\delta=1 / \tau_{c}$. O perfil espectral de cada tipo de fonte é caracterizado pela frequência portadora $\omega_{0}$ e pela largura espectral dada pelo inverso do tempo de coerência $1 / \tau_{c}$. Entretanto, se a fonte de luz fosse estável e descrita por uma onda monocromática, ela não apresenta flutuações e, portanto, o tempo de coerência $\tau_{c} \rightarrow \infty$. Consequentemente, ambas as distribuições convergem à função singular $\delta\left(\omega-\omega_{0}\right)$. O resultado da eq.(1.27) é conhecido como o Teorema de Wiener-Khinthchine. Ele permite fazer o mapeamento entre a função de correlação no domínio temporal de uma variável aleatória estacionária e a sua densidade espectral no domínio da frequência [52]. Para escrevê-lo de uma forma geral, consideremos a variável aleatória $z(t)$ estacionaria cuja média é $\langle z(t)\rangle=0$ e cuja função de autocorrelação temporal é invariante por translação temporal tal que $\langle z(t) z(t+\tau)\rangle=V(\tau)[53]$. O 
resultado do teorema demonstra que a densidade espectral (ou também chamado espectro de potências) é dada por

$$
S(\omega)=\frac{1}{2 \pi} \int_{-\infty}^{\infty}\langle z(t) z(t+\tau)\rangle e^{-i \omega \tau} d \tau
$$

Isto é: dada uma variável aleatória $z(t)$ com média nula, a sua densidade espectral $S(\omega)$ corresponde à transformada de Fourier da função de auto-correlação $\langle z(t) z(t+\tau)\rangle$. Em termos da transformada de Fourier temos

$$
\left\langle z^{*}(\omega) z\left(\omega^{\prime}\right)\right\rangle=S(\omega) \delta\left(\omega-\omega^{\prime}\right)
$$

Entretanto, quando a variável $z(t)$ possui uma média $\langle z(t)\rangle \neq 0$, então consideramos a forma linear $z(t)=\bar{z}+\delta z(t)$ em que $\langle\delta z(t)\rangle=0$, de modo que a densidade espectral é dada por

$$
S(\omega)=\frac{1}{2 \pi} \int_{-\infty}^{\infty}\left[\bar{z}^{2}+\langle\delta z(t) \delta z(t+\tau)\rangle\right] e^{-i \omega \tau} d \tau=\bar{z}^{2} \delta(\omega)+\tilde{S}(\omega)
$$

onde definimos

$$
\tilde{S}(\omega)=\frac{1}{2 \pi} \int_{-\infty}^{\infty}\langle\delta z(t) \delta z(t+\tau)\rangle e^{-i \omega \tau} d \tau=\left\langle\delta \tilde{z}(\omega) \delta \tilde{z}^{*}(\omega)\right\rangle
$$

o qual é chamado espectro de ruído, e somente difere do espectro de potências na frequência zero. Neste caso a densidade espectral $S(\omega)$ apresenta uma singularidade em $\omega=0$ representada pela função delta de Dirac, devido ao valor médio $\bar{z}$. Para $\omega>0$ a distribuição espectral é proporcional à variância da flutuação $\delta z(\omega)$, ou seja, $S(\omega)$ e $\tilde{S}(\omega)$ carregam a mesma informação espectral. Assim, uma amplitude $z(t)$ com flutuações aleatórias no tempo, como é representada na figura 1.6.(a), pode ser representada no domínio das frequências como um conjunto de modos densamente populados com uma frequência central denominada portadora, e as bandas laterais cujo valor médio é nulo, como mostra a figura 1.6.(b).

Assim, o fato da variável $z(t)$ apresentar flutuações na sua amplitude, determina a contri- 
(a)

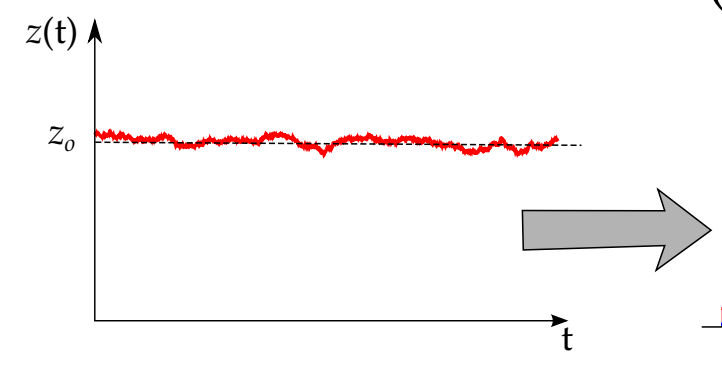

(b)

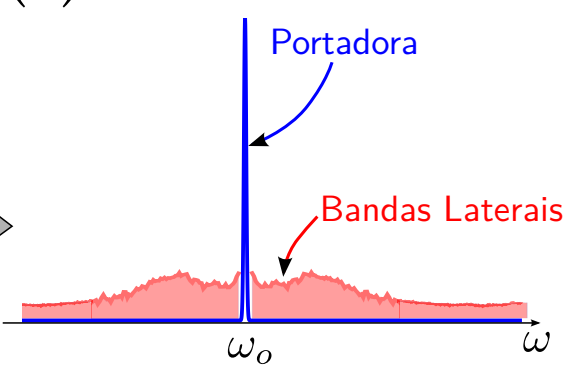

Figura 1.6: (a) Representação temporal de uma variável aleatória estacionária $z(t)$. (b) Representação da densidade espectral de intensidade de um campo com frequência $\omega_{o}$ na portadora (em azul) e ruído das flutuações nas frequências $\omega_{0} \pm \omega$ em vermelho denominadas bandas laterais.

buição das bandas laterais na sua distribuição espectral, tal que $S(\omega)$ não pode ser representada por uma função $\delta(\omega)$ que corresponde a uma única componente espectral $\omega_{0}$. Em particular, para um campo de luz com flutuações de intensidade $\delta I(t)$ em torno a um valor médio, a intensidade é escrita de forma linear como $I(t)=\bar{I}+\delta I(t)$, tal que a sua densidade espectral é dada por

$$
\tilde{S}^{I}(\omega)=\frac{1}{2 \pi} \int_{-\infty}^{\infty}\langle\delta I(t) \delta I(t+\tau)\rangle e^{-i \omega \tau} d \tau
$$

Notemos que a densidade espectral está relacionada à função de correlação medida por Hanbury Brown e Twiss, e definida na eq. (1.18).

Temos definido então a densidade espectral para uma única variável. Entretanto, ao considerar um sistema bipartite, podemos definir o espectro de ruído cruzado e simetrizado como

$$
\tilde{S}_{i j}^{I}(\omega)=\frac{1}{2 \pi} \int_{-\infty}^{\infty}\left\langle\delta I_{i}(t) \delta I_{j}(t+\tau)\right\rangle_{S} e^{-i \omega \tau} d \tau \quad \omega>0
$$

onde $\left\langle\delta I_{i}(t) \delta I_{j}(t+\tau)\right\rangle_{S}=\left(\left\langle\delta I_{i}(t) \delta I_{j}(t+\tau)\right\rangle+\left\langle\delta I_{j}(t) \delta I_{i}(t+\tau)\right\rangle\right) / 2$. Este espectro de ruído descreve a composição espectral da correlação entre as flutuações $\delta I_{i}(t)$ e $\delta I_{j}(t+\tau)$. Da mesma forma que no domínio temporal, podemos definir a desigualdade de Cauchy-Schwarz no 
domínio da frequência, a partir do espectro de ruído da eq.(1.35), tal que

$$
C(\omega)=\frac{\tilde{S}_{12}^{I}(\omega)}{\sqrt{\tilde{S}_{11}^{I}(\omega) \tilde{S}_{22}^{I}(\omega)}}
$$

onde $C(\omega)$ quantifica a desigualdade e é denominado como o coeficiente de correlação [16, 44], que está delimitado por $0 \leq|C(\omega)| \leq 1$ [51]. Este resultado será o nosso principal instrumento na segunda parte deste trabalho para medir a correlação entre dois feixes de luz ao interagir com um conjunto de átomos frios.

Ao longo desta seção, abordamos as medidas das propriedades estatísticas do campo eletromagnético quando ele apresenta flutuações de intensidade e deslocamentos na sua frequência. No que diz respeito às flutuações de intensidade, foi mostrado como correlação de intensidade para instantes de tempo diferentes podem ser critérios para descrever o caráter estatístico da luz. Por outro lado, vimos como as flutuações de intensidades modificam a distribuição espectral da luz, determinando distribuições não singulares. Contudo, é necessário se aprofundar na descrição estocástica de campos com fase que flutuam de forma aleatória, pois veremos que seu tratamento requer de alguns detalhes que serão revisados na próxima seção.

\subsection{Descrição do campo com flutuações de fase estocástica}

Quando a natureza das flutuações de amplitude e fase de um campo surgem por fenômenos aleatórios, é importante empregar a teoria dos processos estocásticos e a dinâmica estocástica para descrevê-la [54]. Este é o tipo de tratamento que nos será útil para descrever o sistema que será estudado no capítulo 3. Nesta seção faremos então uma breve revisão teórica dos tópicos estritamente necessários da teoria dos processos estocásticos que são empregados neste trabalho. Parte das abordagens aqui apresentadas são baseadas na referência [52].

Ao estudar processos estocásticos lidamos com variáveis aleatórias que descrevem a evolução de um sistema dado. Pela teoria da probabilidade, as variáveis aleatórias têm as- 
sociadas distribuições de probabilidade e seus respectivos momentos estatísticos. Portanto, nos processos estocásticos estuda-se, além da evolução temporal da variável aleatória, que pode nos dar informação de uma única realização de eventos, também a dinâmica da sua distribuição de probabilidade e dos seus momentos, que carregam a evolução temporal das várias realizações de eventos. Começaremos então revisando a equação de Langevin que descreve a evolução temporal de variáveis aleatórias, com a qual determinamos a evolução dos momentos estatísticos.

\subsubsection{Equação de Langevin}

O movimento browniano, observado inicialmente em 1827 por Robert Brown [55], foi um experimento que se colocou como base para o estudo da dinâmica estocástica. Na experiência original se estuda o movimento de grãos de pólen num fluido, de forma que ele percorre uma trajetória irregular, que não é possível descrever pela dinâmica determinística de Newton. Em 1905 Einstein propôs no seu trabalho [56] a primeira solução para descrever este tipo de dinâmica. Ela se fundamenta em considerar de forma estatística o impacto recorrente das partículas do fluido no grão de pólen, obtendo assim uma distribuição estatística a posição da partícula como solução da equação de difusão que ele construiu. A distribuição é dada

por $f(x, t)=\frac{n}{\sqrt{4 \pi D t}} e^{-x^{2} / 4 D t}$ caracterizada pela constante de difusão $D$, o que representa uma distribuição Gaussiana para a posição $x$ num tempo $t$ com largura $\sqrt{4 D t}$ e densidade inicial $n$. Esta função de densidade descreve um processo difusivo típico, cuja evolução no tempo difere da descrição Newtoniana para uma partícula em movimento retilíneo uniforme, pois nele, a partícula avança e recua, se deslocando em menor proporção do que um movimento retilíneo.

Mais tarde, em 1908, Paul Langevin propôs uma abordagem a partir da mecânica de Newton para a dinâmica do movimento Browniano. A dinâmica proposta mistura a contribuição de dois tipos de força: uma forma determinística proporcional à velocidade e que descreve a força de atrito no fluido e a segunda, uma força que representa o caráter aleatório das forças devido às partículas do fluido. Desta forma, Langevin construiu a dinâmica numa dimensão 
para uma partícula de massa $m$ da seguinte forma:

$$
m \frac{d v}{d t}=-\alpha v+F_{a}(t)
$$

onde $\alpha$ representa a constante de atrito e $F_{a}(t)$ a força aleatória. Esta equação é conhecida como a equação de Langevin, que pode ser escrita de forma geral para uma variável aleatória $x$ como

$$
\frac{d x(t)}{d t}=a(x, t)+b(x, t) \zeta(t)
$$

em que $a(x, t)$ e $b(x, t)$ são funções conhecidas segundo o problema especifico, e $\zeta(t)$ representa uma variável aleatória rapidamente flutuante, ou seja, que para $t \neq t^{\prime}$ as variáveis $\zeta(t)$ e $\zeta\left(t^{\prime}\right)$ são estatisticamente independentes. Esta forma geral envolve dois tipos de situações: quando a variável aleatória $\zeta(t)$ altera a dinâmica de forma aditiva tal que $b(x, t)=b(t), \mathrm{o}$ que corresponde ao movimento Browniano; e quando a variável aleatória altera a dinâmica de forma multiplicativa, em que $b(x, t)$ é explicitamente dependente da variável $x(t)$ e que multiplica a variável $\zeta(t)$. Em particular temos interesse neste último caso, porque descreve o tipo de sistemas que trataremos no capítulo 3. No entanto, este tipo de evolução temporal apresenta certas características que impedem o uso do cálculo integral e diferencial convencional. A seguir, mostraremos porque surgem certas dificuldades para integrar no tempo esta evolução.

Consideremos a evolução da variável $x(t)$ da eq.(1.38). Para obter a solução no tempo integramos de tal forma que

$$
x(t)=x\left(t_{0}\right)+\int_{t_{0}}^{t} a(x(s)) d s+\int_{t_{0}}^{t} b(x(s), s) \zeta(s) d s
$$

em que os dois primeiros termos dependem unicamente da variável $x(t)$, enquanto que o terceiro termo é acompanhado pela variável que flutua aleatoriamente $\zeta(t)$. Porém ao calcular a integral do produto, é necessário levar em conta certos critérios. A dificuldade para calcular essa integral pode ser vista da seguinte maneira, como é exemplificado na ref.[52]. A integral $\int b(x(s), s) \zeta(s)$ é definida como o limite das somas parciais $S_{n}=\sum_{i}^{n} A_{i}$ 
como mostra a figura 1.7, tal que

$$
A_{i}=B\left(x\left(t_{i}\right), t_{i}\right) \zeta\left(t_{i}\right) \Delta t
$$

onde $A_{i}$ corresponde a área do retângulo $i$-ésimo entre $t_{i}$ e $t_{i+1}$ que definem o intervalo $\Delta=t_{i+1}-t_{i}$, como mostra a figura 1.7(a). A soma das áreas $S_{n}$ vai determinar o incremento da variável $x$ para um tempo $t_{i+1}$, que denominamos como $x_{i+1}$. No entanto, como a variável $\zeta(t)$ pode flutuar de maneira independente da variável $x$ no intervalo $\Delta t$ (ver figura 1.7(b)), então atribuímos ao intervalo $\Delta t$ um novo elemento de área $A_{i}^{\prime}=B\left(x\left(t_{i}\right), t_{i}\right) \zeta\left(\tau_{i}\right) \Delta t$ em que $t_{i} \leq \tau_{i} \leq t_{i+1}$. Claramente $A_{i}^{\prime} \neq A_{i}$ como mostra a figura (c). Em seguida, considere o tempo $t_{i+1}$ em que o elemento de área que corresponde é $A_{i+1}=B\left(x_{i+1}, t_{i+1}\right) \zeta\left(t_{i+1}\right) \Delta t$, no entanto, ele fica indeterminado. Isto é devido ao fato de que o valor $x_{i+1}$ necessário para determinar $B\left(x_{i+1}, t_{i+1}\right)$, é determinado ora pela contribuição de $A_{i}$ (antes de $\zeta\left(t_{i}\right)$ flutuar) ora pelo elemento $A_{i}^{\prime}$ (depois de $\zeta\left(\tau_{i}\right)$ flutuar). Portanto, essa ambiguidade faz com que $B\left(x_{i+1}, t_{i+1}\right)$ seja indeterminado, e consequentemente, $x_{i+1}$ também.

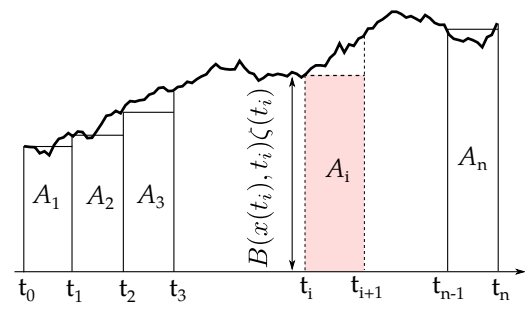

(a)

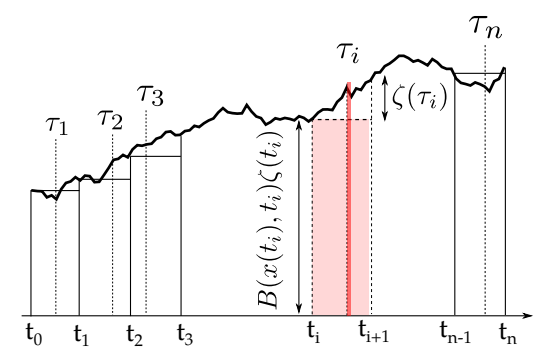

(b)

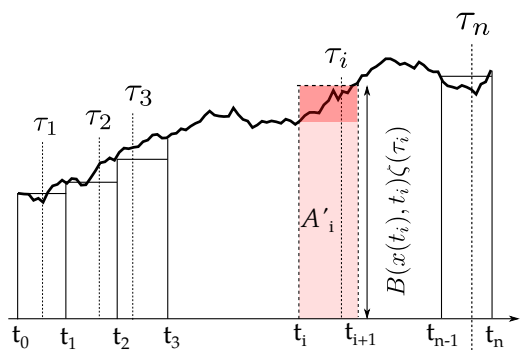

(c)

Figura 1.7: Soma de Riemann para determinar a evolução temporal da variável $x$ sujeita a flutuações $\zeta(t)(a)$ Em vermelho, representamos o elemento de área $A_{i}=B\left(x\left(t_{i}\right), t_{i}\right) \zeta\left(t_{i}\right) \Delta t$ determinada a partir do valor $x_{i}$, para calcular a variável $x_{i+1}$. (b) No entanto, a variável aleatória $\zeta$ pode flutuar no tempo $\tau_{i}$ onde $t_{i}<\tau_{i}<t_{i+1}$, e portanto a área $A_{i}$ flutua também. (c) Para estimar o próximo valor $x_{i+1}$ é necessário computar a soma de Riemann ora com a área $A_{i}$ (antes de $\zeta\left(t_{i}\right)$ flutuar) ora com o novo elemento $A_{i}^{\prime}$ (apos $\zeta\left(t_{i}\right)$ flutuar).

Se a variável aleatória fosse um fator aditivo, ou seja, $B(x(t), t)=B(t)$, não teríamos tal indeterminação. Porém, no caso multiplicativo em que $B$ depende explicitamente da variável estocástica $x(t)$, é necessário tomar uma convenção na escolha do $t_{i}$ em que será 
calculado o elemento de área $A_{i}$, e assim determinar a integral da eq.(1.42).

Essa ambiguidade foi abordada por Stratonovich e Itô de formas diferentes. Stratonovich optou por considerar o valor médio, tal que a integral de forma discreta seria descrita por

$$
S_{n}=\sum_{i}^{n} B\left(\left(x\left(t_{i}\right)+x\left(t_{i+1}\right)\right) / 2, t_{i}\right) \zeta\left(t_{i}\right) \Delta t
$$

enquanto que Itô optou pelo valor antes de ter uma flutuação da variável $\zeta(t)$

$$
S_{n}=\sum_{i}^{n} B\left(x\left(t_{i}\right), t_{i}\right) \zeta\left(t_{i}\right) \Delta t
$$

Como podemos discernir entre essas duas abordagens ao estudar um sistema físico? $\mathrm{Na}$ referência [57] se discute quais são as condições que um sistema físico precisa para definir qual abordagem adotar. No caso do estudo de um sistema físico influenciado por ruído externo, a abordagem mais apropriada pode ser a de Stratonovich. Exemplos disso podem ser: um circuito elétrico com um gerador de ruído externo acoplado com o circuito, sinal de ruído entrando em uma linha de transmissão, crescimento de espécies com influencia do clima, entre outros.

Por outro lado, quando o sistema apresenta um ruído de caráter interno, que não pode ser desligado de forma arbitrária, então as flutuações do sistema podem ser descritas satisfatoriamente pela interpretação de Itô. Exemplos disso são as reações químicas, a emissão e absorção de luz, crescimento de populações, entre outros.

A seguir mostraremos algumas propriedades do calculo de Itô, fundamentais para o estudo do sistema atômico que será apresentado no capítulo 3.

\subsubsection{Dinâmica de variáveis estocásticas: abordagem mediante cálculo de Itô}

Seguiremos aqui a descrição para integração e diferenciação de variáveis estocásticas conforme a referência [52]. A evolução temporal da variável estocástica $x(t)$ descrita na eq.(1.42) 
carrega a dificuldade de lidar com variáveis aleatórias $\zeta(t)$ que não são diferenciáveis e, portanto, as regras do cálculo integral convencional não são aplicáveis.

Para contornar esta situação, Itô reescreve essa integral em termos de processo de Wiener. Um processo de Wiener é aquele que descreve um processo difusivo que depende de uma variável $W(t)$, como mostra a figura 1.8. Uma das características principais de um processo

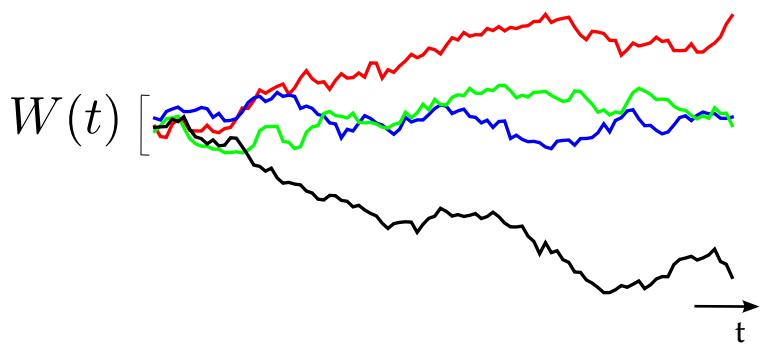

Figura 1.8: Quatro trajetórias simulando um processo de Wiener. Estas são as trajetórias típicas da posição de um movimento Browniano.

de Wiener $W(t)$ é o seu caráter Markoviano. Consideremos aqui um processo estocástico onde podemos medir valores $w_{0}, w_{1}, w_{2}, \ldots, w_{n}$ da variável $W(t)$ no tempos $t_{0}, t_{1}, t_{2}, \ldots, t_{n}$, tal que o processo fique completamente definido pela distribuição de probabilidade conjunta $p\left(w_{0}, t_{0} ; w_{1}, t_{1} ; w_{2}, t_{2} ; \ldots ; w_{n}, t_{n}\right)$. A probabilidade condicional em geral é dada por

$$
p\left(w_{n+1}, t_{n+1} \mid w_{0}, t_{0} ; w_{1}, t_{1} ; w_{2}, t_{2} ; \ldots ; w_{n}, t_{n}\right)=\frac{p\left(w_{0}, t_{0} ; w_{1}, t_{1} ; w_{2}, t_{2} ; \ldots ; w_{n}, t_{n} ; w_{n+1}, t_{n+1}\right)}{p\left(w_{0}, t_{0} ; w_{1}, t_{1} ; w_{2}, t_{2} ; \ldots ; w_{n}, t_{n}\right)}
$$

que corresponde à probabilidade da variável $W(t)$ tomar o valor $w_{n+1}$ no tempo $t_{n+1}$, após ter tomado os valores $w_{0}$ no tempo $t_{0}, w_{1}$ no tempo $t_{1}, w_{2}$ no tempo $t_{2} \cdots$ e $w_{n}$ no tempo $t_{n}$. Para um processo Markoviano, a probabilidade condicional depende somente do momento imediatamente anterior tal que

$$
p\left(w_{n+1}, t_{n+1} \mid w_{0}, t_{0} ; w_{1}, t_{1} ; w_{2}, t_{2} ; \ldots ; w_{n}, t_{n}\right)=p\left(w_{n+1}, t_{n+1} \mid w_{n}, t_{n}\right)
$$

Assim, se o processo $p\left(w_{0}, t_{0} ; w_{1}, t_{1} ; w_{2}, t_{2} ; \ldots ; w_{n}, t_{n}\right)$ for Markoviano, então ele pode ser es- 
crito como

$$
\begin{array}{r}
p\left(w_{0}, t_{0} ; w_{1}, t_{1} ; \ldots ; w_{n}, t_{n} ; w_{n+1}, t_{n+1}\right)=p\left(w_{n+1}, t_{n+1} \mid w_{n}, t_{n}\right) p\left(w_{n}, t_{n} \mid w_{n-1}, t_{n-1}\right) \\
\cdots \times p\left(w_{1}, t_{1} \mid w_{0}, t_{0}\right) p\left(w_{0}, t_{0}\right)
\end{array}
$$

Em particular para um processo de Wiener, a sua densidade de probabilidade condicional é análoga à de um processo de difusão, tal que

$$
p\left(w, t \mid w_{0}, t_{0}\right)=\frac{1}{\sqrt{2 \pi\left(t-t_{0}\right)}} e^{-\left(w-w_{0}\right)^{2} / 2\left(t-t_{0}\right)}
$$

que representa uma gaussiana com média e variância

$$
\begin{aligned}
\langle W(t)\rangle & =w_{0} \\
\left\langle\left[W(t)^{2}-w_{0}^{2}\right]\right\rangle & =t-t_{0}
\end{aligned}
$$

onde seu perfil na condição inicial $t=t_{0}$ corresponde à de uma função $p\left(w, t_{0} \mid w_{0}, t_{0}\right)=$ $\delta\left(w-w_{0}\right)$, que se alarga à medida que evolui no tempo em torno do seu valor médio segundo (1.46). Portanto, de acordo com a eq.(1.45), a probabilidade conjunta de um processo de Wiener é dada por

$$
p\left(w_{0}, t_{0} ; w_{1}, t_{1} ; \ldots ; w_{n}, t_{n} ; w_{n+1}, t_{n+1}\right)=\prod_{i=0}^{n} \frac{e^{-\left(w_{i+1}-w_{i}\right)^{2} / 2\left(t_{i+1}-t_{i}\right)}}{\sqrt{2 \pi\left(t_{i+1}-t_{i}\right)}} p\left(w_{0}, t_{0}\right)
$$

Se definirmos os intervalos $\Delta W_{i}=W\left(t_{i+1}\right)-W\left(t_{i}\right)=w_{i+1}-w_{i}$ e $\Delta t_{i}=t_{i+1}-t_{i}$ então temos uma probabilidade conjunta para incrementos de Wiener $\Delta w_{i}$ tal que

$$
p\left(\Delta w_{1}, t_{1} ; \ldots ; \Delta w_{n}, t_{n} ; \Delta w_{n+1}, t_{n+1}\right)=\prod_{i=0}^{n} \frac{e^{-\left(\Delta w_{i}\right)^{2} / 2\left(\Delta t_{i}\right)}}{\sqrt{2 \pi\left(\Delta t_{i}\right)}} p\left(w_{0}, t_{0}\right)
$$

onde pelo fato de ser um processo Markoviano os incrementos $\Delta w_{i}$ são mutuamente inde- 
pendentes. Isto faz com que a média e a variância dos incrementos sejam

$$
\begin{aligned}
\left\langle\Delta W_{i}\right\rangle & =0 \\
\left\langle\Delta W_{i}^{2}\right\rangle & =\Delta t_{i}
\end{aligned}
$$

de modo que um incremento diferencial $d W$ também satisfaz as eqs.(1.51) e (1.52). Assim, o elemento de integração $\zeta(s) d s$ da eq.(1.42) pode ser definido em termos de um incremento de Wiener, tal que $\zeta(t) d t=d W(t)=W(t+d t)-W(t)$, de modo que a sua integração fica expressa como

$$
\int_{0}^{t} b[x(s), s] \zeta(s) d s \rightarrow \int_{0}^{t} b[x(s), s] d W(s)
$$

Portanto, a dinâmica da variável $x(t)$ descrita pela equação de Langevin (1.38), pode ser reescrita em termos dos elementos de Wiener $d W(t)$ da forma

$$
d x=a[x(t), t)] d t+b[x(t), t] d W(t)
$$

É esta a equação estocástica que Itô se propôs integrar, obtendo assim, uma serie de propriedades para o cálculo integral.

\section{Propriedades da integral de Itô}

A seguir vamos listar as principais propriedades da integral de Itô para funções $G(t)$ não antecipantes, ou seja, funções que são independentes de qualquer processo de Wiener $W(t)$. São elas:

- Fórmula da média

$$
\left\langle\int_{t_{0}}^{t} G(s) d W(s)\right\rangle=0
$$

- Fórmula da correlação: seja $G(t)$ e $H(t)$ funções não antecipantes, então

$$
\left\langle\int_{t_{0}}^{t} G(s) d W(s) \int_{t_{0}}^{t} H(t) d W(t)\right\rangle=\int_{t_{0}}^{t} G(s) H(s) d s
$$


- Fórmula da média para a integral com $N$ elementos de integração de Wiener $d W(t)^{2+N}$

$$
\begin{aligned}
\int_{t_{0}}^{t} G(s) d W(s)^{2+N} & =\int_{t_{0}}^{t} G(s) d s \text { para } N=0 \\
& =0 \text { para } N>0
\end{aligned}
$$

- Potências do elemento diferencial $d W(t)$

$$
\begin{aligned}
d W(t)^{2+N} & \equiv d t \text { para } N=0 \\
& \equiv 0 \text { para } N>0
\end{aligned}
$$

\section{Regra de diferenciação de Itô}

Um dos resultados mais importantes do formalismo de Itô para processo estocásticos é a fórmula de diferenciação para função $f$ que depende da variável estocástica $W(t)$. Ela parte da expansão do elemento diferencial

$$
d f[(W(t), t)]=\frac{\partial f}{\partial t} d t+\frac{\partial f}{\partial W} d W(t)+\frac{1}{2} \frac{\partial^{2} f}{\partial W^{2}} d W(t)^{2}+\cdots
$$

e ao manter termos até segunda ordem $d W(t)^{2}$, pela propriedade (1.59) onde $d t^{2} \rightarrow 0$, obtemos

$$
d f[(W(t), t)]=\left(\frac{\partial f}{\partial t}+\frac{1}{2} \frac{\partial^{2} f}{\partial W^{2}}\right) d t+\frac{\partial f}{\partial W} d W(t)
$$

Estendendo esta fórmula para o caso de duas variáveis estocásticas, temos

$$
\begin{aligned}
d f\left[\left(W_{1}(t), W_{2}(t), t\right)\right]= & \left(\frac{\partial f}{\partial t}+\frac{1}{2} \frac{\partial^{2} f}{\partial W_{1}^{2}}+\frac{1}{2} \frac{\partial^{2} f}{\partial W_{2}^{2}}+\frac{1}{2} \frac{\partial^{2} f}{\partial W_{1} \partial W_{2}}+\frac{1}{2} \frac{\partial^{2} f}{\partial W_{2} \partial W_{1}}\right) d t \\
& +\frac{\partial f}{\partial W_{1}} d W_{1}(t)+\frac{\partial f}{\partial W_{2}} d W_{2}(t)
\end{aligned}
$$




\subsubsection{Exemplo: campo elétrico com excesso de ruído na fase}

Consideremos o caso simples de um campo elétrico $\mathcal{E}(t)$ que oscila harmonicamente, mas que está sujeito a flutuações de fase $W(t)$ que seguem um processo de Wiener. Nessa situação, o campo pode ser escrito como

$$
\mathcal{E}(t)=\mathcal{E}_{0} e^{i[\omega t+\sqrt{2 \gamma} W(t)]}
$$

onde $\omega$ é a frequência de oscilação livre e $\gamma$ é a taxa de difusão da fase. Pela regra de diferenciação de Itô (1.61), a dinâmica é descrita como

$$
d \mathcal{E}(t)=(i \omega-\gamma) \mathcal{E}(t) d t+i \sqrt{2 \gamma} \mathcal{E}(t) d W(t) .
$$

Assim, ao calcular o valor médio da amplitude, temos que $\langle d W(t)\rangle=0$ segundo a eq.(1.51) e, portanto, temos que $d\langle\mathcal{E}(t)\rangle=\exp (i \omega-\gamma) d t$. Desta forma, a evolução temporal do valor médio é $\langle\mathcal{E}(t)\rangle=\langle\mathcal{E}(0)\rangle \exp (i \omega-\gamma)$. Isto mostra que para um oscilador harmônico que é perturbado de forma aleatória fora de fase, gera uma atenuação $\gamma$ na sua amplitude. A dinâmica de qualquer função $f(\mathcal{E}(t))$ deverá empregar as propriedades do cálculo de Itô discutidas nesta seção, como por exemplo, o cálculo dos momentos de ordem superior.

Viu-se então a descrição do cálculo integral e diferencial para variáveis estocásticas propostas por Itô. A partir dele, podemos estudar problemas como a interação de campos eletromagnéticos que apresentam flutuações de fase com meios atômicos em condição de transparência, como será tratado no capítulo 3. Mas antes de abordar este tipo de sistema, a seguir faremos uma breve revisão sobre a descrição quântica dos campos para descrever o tipo de luz emitida por um laser, o qual apresenta flutuações intrínsecas de fase e amplitude. No entanto, essas flutuações estão limitadas ao mínimo de acordo com os limites dados pela mecânica quântica. Veremos então os estados que apresentam mínima incerteza e a representação de estados com excesso de ruído. 


\subsection{Tópicos da descrição quântica do campo}

O tratamento formal para a descrição quântica da luz pode ser encontrado nas referências [7, 49, 58]. Nelas são feitas descrições detalhadas da quantização do campo eletromagnético e das suas diferentes propriedades. O intuito desta seção é destacar somente alguns pontos das propriedades de alguns dos seus estados, que serão úteis para conceitos analisados no capitulo 4 .

Como qualquer sistema físico, uma das propriedades mais importantes e base para o seu estudo, é a energia que ele possui, ou seja, o hamiltoniano que o descreve. Assim, na descrição quântica, a energia do campo eletromagnético é descrita pelo hamiltoniano

$$
H=\sum_{i} \hbar\left[\hat{p}_{i}^{2}+\omega_{i} \hat{q}_{i}^{2}\right]=\sum_{i} \hbar \omega_{i}\left(\hat{a}_{i}^{\dagger} \hat{a}_{i}+\frac{1}{2}\right)
$$

onde $\hat{q}_{i}$ e $\hat{p}_{i}$ são os observáveis do campo eletromagnético associados ao modo $i$-ésimo. Vale destacar que o hamiltoniano (1.66) corresponde ao hamiltoniano de vários osciladores harmônicos que oscilam com frequências diferentes, segundo os modos de oscilação $\omega_{i}$. Os observáveis $\hat{q}_{i}$ e $\hat{p}_{i}$ entretanto, satisfazem relações de comutação

$$
\begin{aligned}
& {\left[\hat{q}_{i}, \hat{p}_{j}\right]=i \hbar \delta_{i, j}} \\
& {\left[\hat{q}_{i}, \hat{q}_{j}\right]=\left[\hat{p}_{i}, \hat{p}_{j}\right]=0}
\end{aligned}
$$

que determinam, portanto, uma relação de incerteza para observáveis do mesmo modo, ou seja, se satisfaz $\Delta^{2} \hat{q}_{i} \Delta^{2} \hat{p}_{i} \geq \hbar^{2} / 4$ onde $\Delta^{2} \hat{O}=\left\langle\hat{O}^{2}\right\rangle-\langle\hat{O}\rangle^{2}$ para qualquer operador $\hat{O}$. Os observáveis apresentam caraterísticas particulares para diferentes estados do campo, como veremos ao longo desta seção.

O hamiltoniano (1.66) está escrito também em termos das amplitudes dos modos $\hat{a}_{i}$ e $\hat{a}_{i}^{\dagger}$ 
que são definidas como

$$
\begin{aligned}
& \hat{a}_{i}=\frac{1}{\sqrt{2 \hbar \omega_{i}}}\left[\omega_{i} \hat{q}_{i}+i \hat{p}_{i}\right] \\
& \hat{a}_{i}^{\dagger}=\frac{1}{\sqrt{2 \hbar \omega_{i}}}\left[\omega_{i} \hat{q}_{i}-i \hat{p}_{i}\right]
\end{aligned}
$$

e que satisfazem as relações de comutação

$$
\left[\hat{a}_{i}, \hat{a}_{j}^{\dagger}\right]=\delta_{i j}
$$

O hamiltoniano do campo determina então a sua dinâmica mediante as equações de Hamilton, que reproduzem a dinâmica descrita pelas equações de Maxwell. Para o caso em que consideramos os campos em um volume cúbico sem a presença de fontes de carga, a solução das equações de Maxwell para os campos $\hat{\mathbf{E}}(\mathbf{r}, t)$ e $\hat{\mathbf{B}}(\mathbf{r}, t)$ é

$$
\begin{aligned}
& \hat{\mathbf{E}}(\mathbf{r}, t)=i \sum_{i}\left(\frac{2 \pi \omega_{i} \hbar}{L^{3}}\right)^{1 / 2}\left(\hat{a}_{i} e^{i\left(\mathbf{k}_{i} \cdot \mathbf{r}-\omega_{i} t\right)} \hat{\mathbf{e}}_{i}-\hat{a}_{i}^{\dagger} e^{-\left(i \mathbf{k}_{i} \cdot \mathbf{r}-\omega_{i} t\right)} \hat{\mathbf{e}}_{i}^{*}\right) \\
& \hat{\mathbf{B}}(\mathbf{r}, t)=i \sum_{i}\left(\frac{2 \pi \hbar}{\omega_{i} L^{3}}\right)^{1 / 2}\left(\hat{a}_{i} e^{i\left(\mathbf{k}_{i} \cdot \mathbf{r}-\omega_{i} t\right)} \mathbf{k}_{i} \times \hat{\mathbf{e}}_{i}-\hat{a}_{i}^{\dagger} e^{-\left(i \mathbf{k}_{i} \cdot \mathbf{r}-\omega_{i} t\right)} \mathbf{k}_{i} \times \hat{\mathbf{e}}_{i}^{*}\right)
\end{aligned}
$$

onde os operadores de amplitude $\hat{a}_{i}$ e $\hat{a}_{i}^{\dagger}$ são interpretados como operadores de criação e aniquilação de fótons, respectivamente. Assim, a ação do operador de campo elétrico $\hat{\mathbf{E}}(\mathbf{r}, t)$ em um dos seus estados representa a criação ou aniquilação de fótons em um dos seus modos clássicos caracterizados pela polarização $\hat{\mathbf{e}}_{i}$, frequência $\omega_{i}$ e modo espacial $\mathbf{k}_{i}$.

Como foi discutido na seção 1.1, podemos definir então as quadraturas do campo como

$$
\begin{aligned}
& \hat{X}_{i}=\hat{a}_{i}+\hat{a}_{i}^{\dagger} \\
& \hat{Y}_{i}=-i\left[\hat{a}_{i}-\hat{a}_{i}^{\dagger}\right]
\end{aligned}
$$

Pela não comutação dos operadores $\hat{a}_{i}$ e $\hat{a}_{i}^{\dagger}$, as quadraturas dos campos satisfazem a relação de comutação $\left[\hat{X}_{i}, \hat{Y}_{i}\right]=2 i$. Como $\hat{X}_{i}$ e $\hat{Y}_{i}$ são operadores hermitianos então são observáveis físicos canonicamente conjugados. 
Uma das principais características que a mecânica quântica introduz na descrição da luz é a incerteza dos observáveis físicos $\hat{X}_{i}$ e $\hat{Y}_{i}$. Ao contrário da descrição clássica, em que as quadraturas dos campos (1.3) são determinadas com absoluta precisão, na descrição quântica, o princípio de incerteza de Heisenberg determina flutuações intrínsecas nas quadraturas, que impedem determiná-las com precisão absoluta simultaneamente, ou seja,

$$
\Delta^{2} \hat{X} \Delta^{2} \hat{Y} \geq 1
$$

Nesta seção, revisaremos algumas características das quadraturas do campo eletromagnético para os estados que satisfazem a mínima incerteza.

\section{Estados de mínima incerteza}

Um dos principais estados do campo eletromagnético que apresenta características unicamente descritas pela mecânica quântica, é o estado de Fock $\left|n_{i}\right\rangle$. Ele é um auto-estado do operador número $\hat{n}_{i}=\hat{a}_{i}^{\dagger} \hat{a}_{i}$ e também um auto-estado do hamiltoniano $H$ da eq.(1.66). Este estado é caracterizado pelo número de fótons $n_{i}$ no modo $i$-ésimo. No caso em que $n_{i}=0$ em todos seus modos, definimos o estado do campo como o estado de vácuo. Entretanto, um dos estados nos quais temos interesse, é o estado coerente. Se considerarmos um único modo do campo eletromagnético, o estado coerente é descrito por $|\alpha\rangle$ e é definido como o auto-estado do operador de aniquilação $\hat{a}$ de um único modo, tal que

$$
\hat{a}|\alpha\rangle=\alpha|\alpha\rangle \quad \rightarrow \quad\langle\alpha| \hat{a}^{\dagger}=\alpha^{*}\langle\alpha|
$$

em que $\alpha$ é uma amplitude complexa arbitrária tal que $\alpha=|\alpha| e^{i \phi}$. O estado coerente se expande na base de estados de Fock $|n\rangle$ da seguinte forma:

$$
|\alpha\rangle=e^{-|\alpha|^{2} / 2} \sum_{n=0}^{\infty} \frac{\alpha^{n}}{\sqrt{n !}}|n\rangle
$$


onde $|n\rangle$ representa o número de excitações ou fótons no modo do campo. O estado coerente é então a superposição de vários desses estados com uma distribuição de Poisson

$$
P(n)=\frac{|\alpha|^{2}}{n !} e^{-|\alpha|^{2}}=\frac{\langle n\rangle}{n !} e^{-\langle n\rangle}
$$

que mede a probabilidade para achar $n$ fótons no estado coerente. Este tipo de distribuição tem como característica que o valor médio de fótons é $\langle n\rangle=|\alpha|^{2}$ e que a variância no número de fótons é proporcional ao valor médio, ou seja, $\left\langle\Delta^{2} \hat{n}\right\rangle=\langle n\rangle=|\alpha|^{2}$.

Uma das características mais importantes do estado coerente é que a variância das quadraturas $\hat{X}$ e $\hat{Y}$ satisfazem a relação de mínima incerteza, ou seja, $\Delta^{2} \hat{X}=\Delta^{2} \hat{Y}=1$. Este valor de mínima incerteza é conhecido como o limite quântico padrão (LQP). Vale notar, que mesmo no estado de vácuo $|\alpha=0\rangle$, ele também satisfaz a condição de mínima incerteza, o que faz com que seja um estado coerente.

Neste ponto, podemos fazer uma comparação entre a descrição do campo clássico e a descrição do campo mediante o estado coerente. No caso clássico mostramos como a amplitude e fase do campo eram definidas de forma precisa. Entretanto, no caso quântico, o valor médio do campo no estado coerente é dado por

$$
\begin{aligned}
\langle\alpha|\hat{\mathbf{E}}(\mathbf{r}, t)| \alpha\rangle & =i\left(\frac{4 \pi \omega^{2}}{L^{3}}\right)^{1 / 2}\left(\alpha e^{i(\mathbf{k} \cdot \mathbf{r}-\omega t)} \mathbf{e}-\alpha^{*} e^{-(i \mathbf{k} \cdot \mathbf{r}-\omega t)} \mathbf{e}^{*}\right) \\
& =\mathcal{E} e^{i(\mathbf{k} \cdot \mathbf{r}-\omega t)} \mathbf{e}+\mathcal{E}^{*} e^{-(i \mathbf{k} \cdot \mathbf{r}-\omega t)} \mathbf{e}^{*}
\end{aligned}
$$

onde definimos $\mathcal{E}=i \sqrt{4 \pi \omega^{2} / L^{3}} \alpha$. Este resultado coincide com a descrição clássica em (1.2), representada na figura 1.9.(a) como um vetor com amplitude $|\mathcal{E}|$ no plano complexo, e como uma onda oscilante na representação temporal. No entanto, diferente do caso clássico, ao considerarmos os momentos de ordem superior, as quadraturas do campo apresentam flutuações intrínsecas de acordo com as variâncias na eq.(1.73). Isto é representado mediante um diagrama de Fresnel no espaço de fase quântico na figura 1.9.(b), onde o estado coerente possui um valor médio $\alpha$ e em torno dele, há uma variância nas suas quadraturas representada pela área vermelha, que coincide com o LQP em linha tracejada. Junto com 
o diagrama de fresnel mostramos uma representação temporal do campo elétrico em um estado coerente. Contudo, o estado coerente da luz é considerado o estado mais próximo da descrição clássica do campo eletromagnético.

(a)

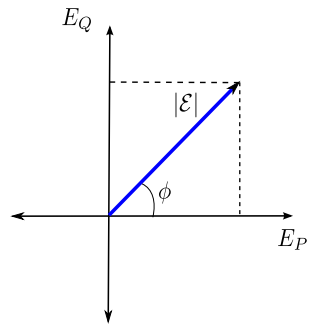

$E(t)$

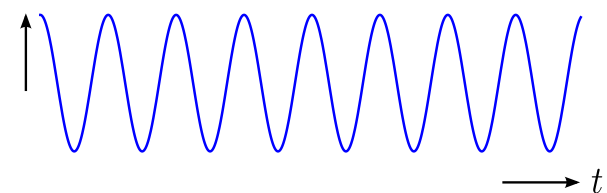

(b)

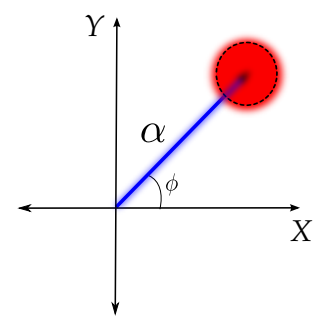

$E(t)$

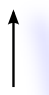

Figura 1.9: (a) Descrição clássica do campo elétrico: a representação temporal do campo é feita por meio de uma onda oscilante bem definida. No plano complexo o campo é descrito por um vetor com componente $E_{P}$ e $E_{Q}$ bem definidos. (b) Descrição de um estado coerente do campo elétrico: a representação temporal do campo é dada por um onda que oscila com amplitude e fase difusa. Na representação de Fresnel, o campo se mostra como um vetor em azul que apresenta incertezas nas suas quadraturas $X$ e $Y$, representadas pelo circulo em vermelho, que coincide com o LQP em linha tracejada.

Além do estado coerente, existe outro tipo de estado que satisfaz a condição de mínima incerteza de Heisenberg (1.73). Ele é denominado estado comprimido, e se caracteriza por que as variâncias das suas quadraturas satisfazem

$$
\begin{array}{ll}
\Delta^{2} \hat{X}>1 & \Delta^{2} \hat{Y}<1 \quad \rightarrow \quad \Delta^{2} \hat{X} \Delta^{2} \hat{Y}=1 \checkmark \\
\Delta^{2} \hat{X}<1 & \Delta^{2} \hat{Y}>1 \quad \rightarrow \quad \Delta^{2} \hat{X} \Delta^{2} \hat{Y}=1 \checkmark
\end{array}
$$

As figuras 1.10.(a) e (b) mostram o diagrama de Fresnel para o estado em que temos compressão na quadratura $X$ ou compressão na quadratura $Y$, respectivamente. Junto ao diagrama de fresnel, temos a representação temporal do campo em cada um desses estados de compressão.

É importante notar que o principio de Heisenberg garante também a possibilidade de $\Delta^{2} \hat{X} \Delta^{2} \hat{Y}>1$. Isto significa que podemos ter fontes de luz em que uma das quadraturas 
(a)

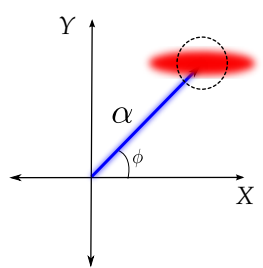

(b)

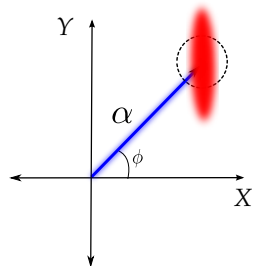

(c)

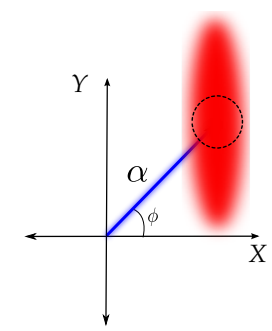

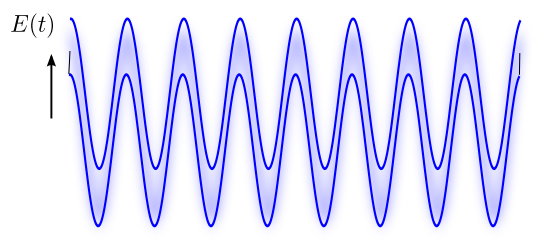

$E(t)$
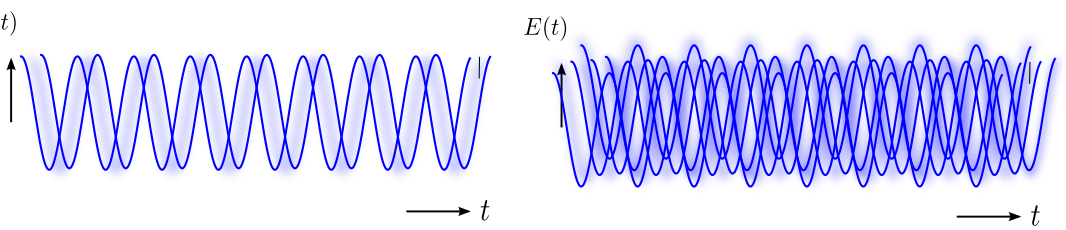

Figura 1.10: Representação do estado comprimido do campo elétrico para o caso em que (a) o campo apresenta uma compressão de incerteza na sua quadratura $Y$ e excesso na sua quadratura $X$ e (b) para o caso em que o campo apresenta um compressão de incerteza na sua quadratura $X$ e excesso na sua quadratura $Y$. (c) Corresponde à representação de um campo com excesso de ruído de fase, que está associado à quadratura $Y$, onde a quadratura $X$ se mantêm ao nível de $L Q P$.

se mantém no nível do LQP, por exemplo $\Delta^{2} \hat{X}=1$, enquanto que a outra quadratura se mantêm acima dele, ou seja, $\Delta^{2} \hat{Y} \gg 1$, como é representado na figura 1.10(c). Isto satisfaz o principio de Heisenberg e corresponde à descrição de um laser de diodo que apresenta excesso de ruído na sua quadratura de fase, enquanto que a quadratura de amplitude se mantêm próxima do LQP. Este tipo de luz é a que será empregadas nos experimentos do capítulo 3.

\subsubsection{Shot-noise}

O limite quântico padrão define o ruído de intensidade intrínseco dos estados coerentes denominado ruído de Shot-noise (em inglês). Este é o ruído intrínseco à chegada dos fótons nos fotodetetores e descrito por uma distribuição de Poisson. Para medi-lo, basta passar o feixe de luz por um divisor de feixes e dois detetores. De maneira formal, consideremos os campos $\hat{a}$ e $\hat{b}$ incidindo num divisor de feixe com transmissão $|t|^{2}$ e reflexão $|r|^{2}$ iguais (deteção balanceada), como mostra a figura 1.11. Em particular, vamos analisar o caso em que o campo $\hat{a}$ se encontra no estado qualquer $|\psi\rangle$ e o campo $\hat{b}$ se encontra no estado de vácuo $|0\rangle$ 


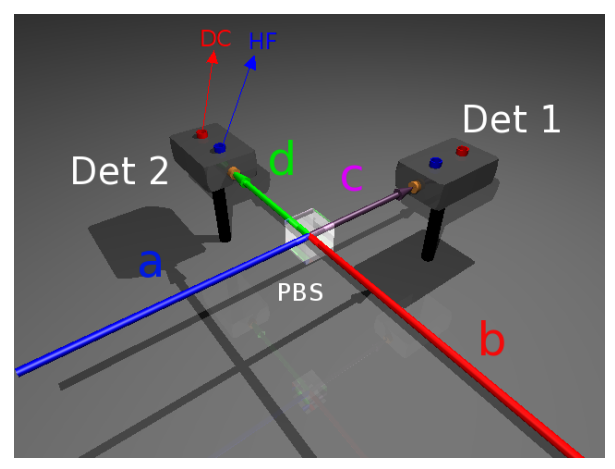

Figura 1.11: Deteção balanceada para calibração de shot-noise

Na saída do divisor, obtemos dois campos $\hat{c}$ e $\hat{d}$, dados por

$$
\begin{aligned}
& \hat{c}=\frac{1}{\sqrt{2}}(\hat{a}+\hat{b}) \\
& \hat{d}=\frac{1}{\sqrt{2}}(\hat{a}-\hat{b})
\end{aligned}
$$

onde o termo negativo na segunda equação corresponde à defasagem que ganha o feixe ao ser refletido. As intensidades das fotocorrentes induzidas pelos campos óticos nos fotodetetores, são proporcionais ao operador número de $\hat{c}$ e $\hat{d}$, tal que

$$
\begin{aligned}
& \hat{I}_{1}=g \hat{d}^{\dagger} \hat{d} \\
& \hat{I}_{2}=g \hat{c}^{\dagger} \hat{c}
\end{aligned}
$$

em $g$ é uma constante de ganho dos detetores (levando em conta amplificações dos circuitos eletrônicos). Nesta situação, em que um feixe está sendo dividido em dois campos com intensidades médias iguais, é de se esperar, do ponto de vista clássico, que a subtração dessas fotocorrentes seja zero e a soma seja igual à intensidade do campo incidente. Intuitivamente isto seria verdade tanto para o valor médio, quanto, para campos com flutuações de intensidade. No entanto, a mecânica quântica mostra que para as flutuações, isto não é necessariamente verdade. A subtração das fotocorrentes não é zero, e seu valor resultante é o Shot noise. Isto fica mais claro, a partir das equações (1.80)-(1.83), com as quais determinamos os 
operadores de soma e subtração

$$
\begin{aligned}
& \hat{I}_{+}=\hat{I}_{1}+\hat{I}_{2}=g\left(\hat{a}^{\dagger} \hat{a}+\hat{b}^{\dagger} \hat{b}\right) \\
& \hat{I}_{-}=\hat{I}_{1}-\hat{I}_{2}=g\left(\hat{a}^{\dagger} \hat{b}+\hat{b}^{\dagger} \hat{a}\right) .
\end{aligned}
$$

Para determinar o ruído de intensidade dos campos, definido como $\left\langle(\Delta \hat{I})^{2}\right\rangle=\left\langle\hat{I}^{2}\right\rangle-\langle\hat{I}\rangle^{2}$, precisamos calcular

$$
\begin{aligned}
& \hat{I}_{+}^{2}=g^{2}\left[\left(\hat{n}_{a}\right)^{2}+\left(\hat{n}_{b}\right)^{2}+2 \hat{n}_{a} \hat{n}_{b}\right], \\
& \hat{I}_{-}^{2}=g^{2}\left[\left(\hat{a}^{\dagger} \hat{b}\right)^{2}+\left(\hat{b}^{\dagger} \hat{a}\right)^{2}+\hat{a}^{\dagger} \hat{a} b \hat{b}^{\dagger}+\hat{a} \hat{a}^{\dagger} \hat{b}^{\dagger} \hat{b}\right] .
\end{aligned}
$$

sendo $\hat{n}_{a}$ e $\hat{n}_{b}$ os operadores número dos feixes $\hat{a}$ e $\hat{b}$, respectivamente. Portanto, o valor médio e o segundo momento dos operadores de soma e subtração são

$$
\begin{aligned}
& \left\langle\hat{I}_{+}\right\rangle=g\left\langle\hat{n}_{a}\right\rangle_{a} \\
& \left\langle\hat{I}_{-}\right\rangle=0 \\
& \left\langle\hat{I}_{+}^{2}\right\rangle=g^{2}\left[\left\langle\left(\hat{a}^{\dagger} \hat{a}\right)^{2}\right\rangle_{a}\right] \\
& \left\langle\hat{I}_{-}^{2}\right\rangle=g^{2}\left[\left\langle\hat{n}_{a}\right\rangle_{a}\right]
\end{aligned}
$$

em que consideramos o estado do campo $\hat{b}$ como vácuo $|0\rangle$. Pelo fato de que $\left\langle\hat{n}_{a}\right\rangle_{a}=I_{a}=$ $I_{1}+I_{2}$, sendo esta a intensidade média do campo de entrada, então finalmente obtemos a variância da soma e da subtração:

$$
\begin{array}{ll}
\left\langle\left(\Delta \hat{I}_{+}\right)^{2}\right\rangle=g^{2}\left\langle\left(\Delta \hat{n}_{a}\right)^{2}\right\rangle & \text { Ruído de intensidade do campo } \hat{a} \\
\left\langle\left(\Delta \hat{I}_{-}\right)^{2}\right\rangle=g^{2} I_{a} & \text { Ruído de shot-noise }
\end{array}
$$

Assim, ao medir a subtração das fotocorrentes determinamos então o Shot-noise que define o ruído de referência com o qual comparamos o ruído da fonte que formos empregar. Isto se faz calculando $\left\langle\left(\Delta \hat{I}_{+}\right)^{2}\right\rangle /\left\langle\left(\Delta \hat{I}_{-}\right)^{2}\right\rangle=\left\langle\left(\Delta \hat{n}_{a}\right)^{2}\right\rangle / I_{a}$ onde comparamos o ruído do campo, dado 
pela soma $\left\langle\left(\Delta \hat{I}_{+}\right)^{2}\right\rangle$, e o shot-noise, dado pela subtração $\left\langle\left(\Delta \hat{I}_{-}\right)^{2}\right\rangle$. Por exemplo, se tivermos um campo coerente, então $\left\langle\left(\Delta \hat{n}_{a}\right)^{2}\right\rangle / I_{a}=1$. Entretanto, um campo com excesso de ruído apresentará uma relação $\left\langle\left(\Delta \hat{n}_{a}\right)^{2}\right\rangle / I_{a}>1$. Finalmente, um campo com compressão de ruído terá então $\left\langle\left(\Delta \hat{n}_{a}\right)^{2}\right\rangle / I_{a}<1$.

\section{Conclusões do capítulo}

Neste primeiro capítulo temos revisadas três descrições do campo eletromagnético. A primeira é a descrição clássica, que permitiu introduzir as principais características do campo ao ser descrita como uma onda monocromática. A segunda é uma descrição ainda clássica, mas que considera o caráter aleatório da emissão das fontes de luz, levando em conta as possíveis flutuações na sua fase e amplitude. Nessa descrição introduzimos duas propriedades que permitem analisar o caráter estatístico da luz: a função de correlação e a densidade espectral. Além dessas propriedades, introduzimos também o formalismo de Itô para variáveis que seguem uma dinâmica estocástica. A função de correlação e a densidade espectral serão objeto de estudo no capítulo 3, para sistemas em que a luz interage com meios atômicos. Finalmente, a terceira descrição do campo revisada neste capítulo, foi a descrição quântica. Nela mostramos as principais características do campo para um estado coerente, um estado comprimido e um estado com excesso de ruído de fase. 


\section{CAPÍTULO 2}

\section{INTERAÇÃO ENTRE A LUZ E A MATÉRIA}

2.1 Polarização do meio atômico . . . . . . . . . . . . . . . . . . 41

2.2 Descrição microscópica: Matriz densidade . . . . . . . . . . 43

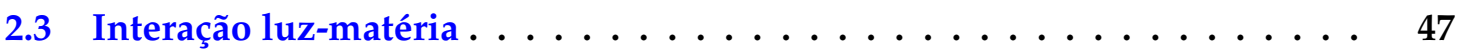

2.4 Força devido à interação luz-átomos . . . . . . . . . . . . . 52

A descrição de uma interface entre a luz e a matéria se fundamenta na possibilidade de polarizar a matéria mediante radiação. Quando um meio atômico é polarizado, os estados internos atômicos são transformados de acordo com as características da luz, isto é, frequência, intensidade e polarização. Um dos grandes desafios é encontrar técnicas para explorar os diferentes graus de polarização de um meio atômico (linear e não-linear) que transforme os estados atômicos de forma coerente, gerando toda uma série de fenômenos aplicados em protocolos de armazenamento, processamento e transporte de informação na área de computação e informação quântica.

Nos últimos 40 anos têm-se estudado vários fenômenos da ótica linear e não-linear, desenvolvendo técnicas para manipulação coerente de estados atômicos [20,59]. Qual a importância da manipulação coerente dos estados atômicos no contexto de informação quântica? Entre várias das razões para focarmos nossa atenção no estudo da interação entre a luz e a 
matéria, está a de ela estar presente nas experiências feitas para testar fundamentos de áreas como a mecânica quântica, a física atômica e a ótica. Além disso, a manipulação de estados atômicos é essencial em inúmeros fenômenos em que o acoplamento fóton-átomo pode induzir um acoplamento efetivo fóton-fóton, fundamental para a maioria dos sistemas em informação quântica. Um primeiro exemplo é a manipulação coerente dos estados atômicos em que se codifica a informação. Isto possibilita a realização de transformações unitárias desses estados, como é o caso de íons armadilhados para implementação de comportas lógicas [60]. Um segundo exemplo são as amostras de átomos frios mediante o aprisionamento e resfriamento de átomos neutros. Trata-se de um dos principais sistemas atômicos, a partir do qual se desdobram algumas experiências, como das memórias quânticas no protocolo de repetidores quânticos [30], a eletrodinâmica quântica em cavidades (QED em inglês)[61] e a geração de emaranhamento ${ }^{1}$ entre estados atômicos e da luz [31]. Também temos manipulação de estados atômicos em amostras à temperatura ambiente e dispositivos de estado sólido [19], os quais são empregados para protocolos de memórias quânticas, geração de emaranhamento [25] e medidas quânticas não destrutivas (QND) [20]. Estes e muitos outros sistemas fazem da interface luz-matéria um dos principais objetos de estudo para entender mecanismos de processamento e armazenamento de informação para aplicações em computação quântica.

Na primeira parte deste capítulo faremos uma breve revisão da polarização linear de um meio atômico. Em seguida descreveremos o hamiltoniano de interação entre campos eletromagnéticos acoplados com átomos de $\mathrm{N}$ níveis atômicos e apresentaremos suas equações de Bloch. Na última parte faremos uma breve revisão sobre as forças que a luz exerce na matéria devido à sua interação.

\footnotetext{
${ }^{1} \mathrm{O}$ emaranhamento é uma das principais propriedades dos sistemas quânticos com mais de dois subsistemas e que representa um dos pilares da informação e computação quântica [6].
} 


\subsection{Polarização do meio atômico}

A propagação da luz em um meio atômico faz não só com que o meio se polarize, mas também com que algumas das suas propriedades sejam modificadas reciprocamente. Dependendo da resposta que o meio possa apresentar ao interagir com um campo eletromagnético $\mathbf{E}(\mathbf{r}, t)$, pode-se gerar uma ampla variedade de fenômenos óticos. De acordo com a eq.(1.2), um campo eletromagnético que oscila com frequência $\omega$ e se propaga em direção k é descrito como

$$
\mathbf{E}(\mathbf{r}, t)=\tilde{\mathcal{E}}_{0} e^{i(\mathbf{k} \cdot \mathbf{r}-\omega t+\phi)} \hat{\mathbf{e}}(\theta, \epsilon)+\text { c.c }
$$

em que $\tilde{\mathcal{E}}_{0}$ representa a amplitude real (que em geral varia de acordo com a posição), $\phi$ é a fase do campo e ê $(\theta, \epsilon)$ é o vetor de polarização do campo com ângulo e elipticidade de polarização $\theta$ e $\epsilon$, respectivamente [46]. Esta polarização satisfaz a condição de ortogonalidade $\mathbf{k} \cdot \hat{\mathbf{e}}(\theta, \epsilon)=0$. Em geral, quando o campo eletromagnético se propaga no meio atômico, pode sofrer modificações de ganho ou absorção na sua amplitude $\tilde{\mathcal{E}}+\Delta \tilde{\mathcal{E}}$, de defasagem $\phi+\Delta \phi$, de polarização $\theta+\Delta \theta$ ou de elipticidade $\epsilon+\Delta \epsilon$, como mostra a figura 2.1.

Para descrever como a propagação do campo pode alterar as propriedades óticas do meio, partimos das equações de Maxwell em meios dielétricos, das quais pode-se obter a equação de propagação do campo [45, 62]

$$
\nabla^{2} \mathbf{E}(\mathbf{r}, t)-\frac{1}{c^{2}} \frac{\partial^{2} \mathbf{E}(\mathbf{r}, t)}{\partial^{2} t}=\frac{1}{c^{2}} \frac{\partial^{2} \mathbf{P}(\mathbf{r}, t)}{\partial^{2} t}
$$

A relação entre o campo e a polarização $\mathbf{P}(\mathbf{r}, t)$ pode ser representada de forma geral a partir de uma resposta linearizada mais uma parte não-linear na amplitude do campo, tal que

$$
\mathbf{P}(\mathbf{r}, t)=\chi^{(1)} \mathbf{E}(\mathbf{r}, t)+\mathbf{P}_{N L}(\mathbf{r}, t)
$$

em que $\chi^{(1)}$ corresponde à susceptibilidade linear ${ }^{3}$ do meio e $\mathbf{P}_{N L}$ representa sua natureza não-

\footnotetext{
${ }^{2}$ A sigla c.c, denota o complexo conjugado

${ }^{3}$ Em geral a susceptibilidade é um tensor de segunda ordem, no entanto, neste caso vamos considerá-lo
} 


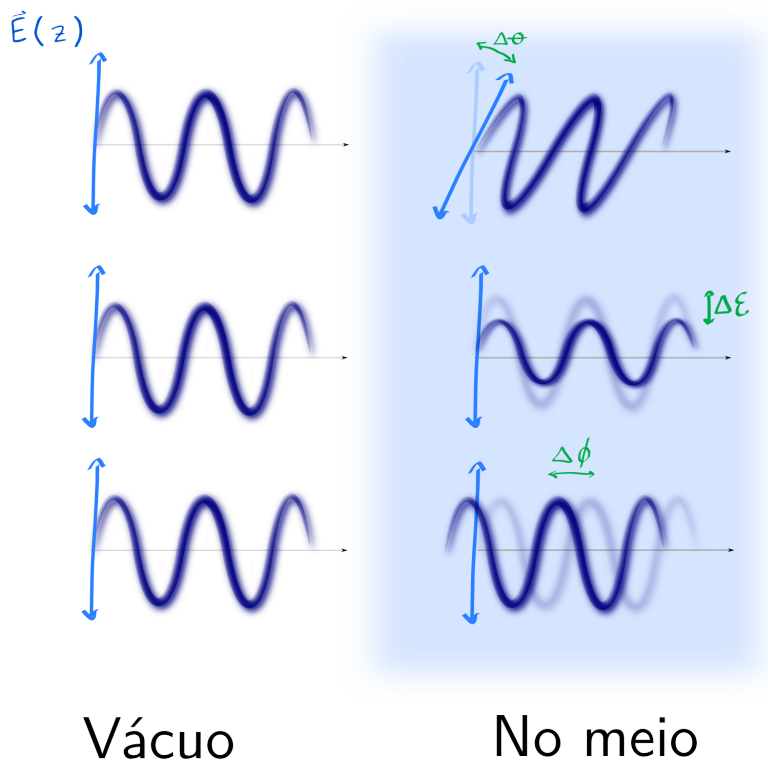

Figura 2.1: (Esquerda) Representação do campo elétrico se propagando no vácuo. (Direita) Modificações que em geral podem ocorrer nas propriedades do campo ao se propagar por um meio atômico. Por exemplo pode ter uma modificação $\Delta \theta$ no ângulo de polarização, $\Delta \tilde{\mathcal{E}}$ na amplitude ou $\Delta \phi$ na fase do campo.

linear. A componente linear da polarização, em geral, pode ter uma parte real associada à dispersão do campo no meio, ou seja, é a componente que gera defasagens $\Delta \phi$ no campo, e uma parte imaginária associada à absorção ou ao ganho do meio. Entretanto, no que diz respeito à resposta não-linear de um meio, ela ocorre quando os campos de luz interagentes são intensos suficiente para modificar a própria resposta de dispersão e absorção do meio. Isto possibilita a geração de novos campos de luz como, por exemplo, a geração de segundo harmônico ou mistura de quatro ondas [62]. No entanto, nesta seção nos restringiremos a revisar unicamente a resposta linear.

Consideremos um meio que possui unicamente susceptibilidade linear, ignorando qualquer contribuição não-linear do meio. Esta susceptibilidade linear pode ser escrita como

$$
\chi^{(1)}=\operatorname{Re} \chi^{(1)}+i \operatorname{Im} \chi^{(1)},
$$

de modo que, pela definição (2.3), a polarização do campo é escrita como $\mathbf{P}_{L}(\mathbf{r}, t)=\mathbf{P}_{R e}(\mathbf{r}, t)+$ como um escalar efetivo, determinado pela polarização e direção de propagação [62]. 
$i \mathbf{P}_{I m}(\mathbf{r}, t)$, em que $\mathbf{P}_{R e}(\mathbf{r}, t)=\operatorname{Re} \chi^{(1)} \mathbf{E}(\mathbf{r}, t)$ corresponde à polarização do meio que oscila em fase com o campo e $\mathbf{P}_{I m}(\mathbf{r}, t)=\operatorname{Im} \chi^{(1)} \mathbf{E}(\mathbf{r}, t)$ representa a polarização do meio que oscila em quadratura como o campo, ou seja, oscila com uma fase $\pi / 2$ com relação à componente real $\mathbf{P}_{R e}$. Se considerarmos que o campo se propaga em direção $\hat{\mathbf{z}}$ e levarmos em conta a componente real e imaginária da polarização na eq.(2.4), a propagação descrita em (2.2) é reduzida a

$$
\frac{\partial^{2} \mathbf{E}(z, t)}{\partial^{2} z}+k^{2}\left(1+\operatorname{Re} \chi^{(1)}\right) \mathbf{E}(z, t)+i k^{2} \operatorname{Im} \chi^{(1)} \mathbf{E}(z, t)=0,
$$

A parte real da susceptibilidade modifica o número de onda $k=\omega / c$ (definido no vácuo) em $k n$ onde $n=\sqrt{\left(1+\operatorname{Re} \chi^{(1)}\right)}$ é definido como índice de refração do meio.

A equação de propagação do campo (2.5) admite uma solução de ondas planas, tal que $\mathbf{E}(\mathbf{z}, t)$ é

$$
\mathbf{E}(z, t)=\tilde{\mathcal{E}}_{o} e^{-(k / 2) \operatorname{Im} \chi^{(1)} z} e^{i(k n z-\omega t+\phi)} \hat{\mathbf{e}}(\theta, \epsilon)+\text { c.c. }
$$

onde $\tilde{\mathcal{E}}_{o}$ é amplitude do campo independente da posição. Esta solução mostra como o campo, ao entrar no meio, oscila com certa defasagem ${ }^{4} k n$ com respeito à propagação no vácuo, e à medida que se propaga oscilando e polarizando o meio, a sua amplitude é amplificada ou atenuada pelo fator $\operatorname{Im} \chi^{(1)}$. Isto mostra que a resposta do meio fora de fase com respeito à oscilação do campo $\operatorname{Im} \chi^{(1)}$ pode se opôr, aumentando ou diminuindo a amplitude de oscilação [46]. Desta forma, mostramos uma descrição simples de como o meio pode alterar não só a fase do campo $\phi \rightarrow \phi+\Delta \phi$, mas também a sua amplitude $\tilde{\mathcal{E}} \rightarrow \tilde{\mathcal{E}}+\Delta \tilde{\mathcal{E}}$.

\subsection{Descrição microscópica: Matriz densidade}

O que determina o tipo de resposta de um meio atômico? Primeiramente, vai depender das simetrias espaciais do meio, da intensidade dos campos e de como a luz se propaga no meio. Como descrevemos essa resposta? A discussão anterior corresponde à descrição ma-

\footnotetext{
${ }^{4} \mathrm{Na}$ eq.(2.6) o índice de refração $n$ esta em vermelho somente indicar a diferença com a solução do campo no vácuo da eq.(2.1). A longo do trabalho grifaremos algumas variáveis para facilitar a visualização.
} 
croscópica da interação entre a luz e a matéria representada pela susceptibilidade do meio $\chi^{(n)}$. No entanto, para determinar como é a susceptibilidade de um meio atômico especifico, é necessário fazer uma descrição microscópica. A mecânica quântica mostra que, em geral, os átomos se caracterizam por terem níveis discretos de energia associados a estados de energia, momento angular, spin eletrônico e nuclear. Esses estados formam uma base no espaço de Hilbert. O operador de matriz densidade é o objeto que contem toda a informação dos estados de um sistema quântico.

Em particular, a matriz densidade para um átomo com $n$ níveis de energia é descrita na base $|n\rangle$ como

$$
\rho=\sum_{n, m} \rho_{n m}|n\rangle\langle m|=\left(\begin{array}{ccccc}
\rho_{11} & \rho_{12} & \rho_{13} & \ldots & \rho_{1 n} \\
\rho_{21} & \rho_{22} & \rho_{23} & \ldots & \rho_{2 n} \\
\rho_{31} & \rho_{32} & \rho_{33} & \ldots & \rho_{3 n} \\
\vdots & \vdots & \vdots & \vdots & \vdots \\
\rho_{n 1} & \rho_{n 2} & \rho_{n 3} & \ldots & \rho_{n n}
\end{array}\right)
$$

em que, pela normalização, temos $\operatorname{Tr} \rho=1$. Os elementos $\rho_{n n}$ descrevem então as populações de cada um dos níveis, enquanto que para $n \neq m$ os elementos $\rho_{n m}$ representam as coerências atômicas. Elas são o resultado das superposições entre os níveis atômicos, as quais podem ser excitadas pelo acoplamento com campos externos (luz, campo magnéticos e colisões, entre os mais comuns).

A partir da matriz de densidade $\rho$ podemos obter a polarização de um conjunto de átomos independentes como o valor médio da soma dos momentos dipolares, tal que

$$
\mathbf{P}=\bar{n} \operatorname{Tr}\{\rho \mathbf{d}\}
$$

em que $\mathbf{d}$ corresponde ao operador do momento dipolar elétrico do meio e $\bar{n}$ à densidade do meio. Assim, a matriz densidade é o objeto que descreve a resposta da polarização. Dado um sistema atômico com o seu hamiltoniano, a polarização é determinada quando a evolução temporal dos estados atingem o regime estacionário. 
Uma interface luz-matéria explora então a possibilidade de preparar o meio em estados específicos, isto é, o acoplamento com luz pode induzir a uma configuração em que, por exemplo, $\rho_{i i} \approx 1$ e $\rho_{j j} \approx 0$ com $i \neq j$. As armadilhas magneto-ópticas são um exemplo de preparação coerente de estados atômicos. No entanto, é mais interessante ter a possibilidade de criar superposições $\rho_{n m}$ em uma escala de tempo controlável. A partir desta superposição, as propriedades codificadas na luz poderiam ser mapeadas e armazenadas de forma coerente nessas superposições atômicas. O meio atômico mais simples, que poderíamos analisar como uma interface de luz-matéria, seria um sistema de dois níveis como mostra a figura 2.2.(a). Neste tipo de meio, a resposta linear é dominante e caracterizada pelo índice de refração e de absorção de luz. No entanto, a única superposição de níveis possível é a do estado fundamental $|1\rangle$ e a do estado excitado $|2\rangle$, que é constantemente excitada devido ao acoplamento do campo. Porém, a emissão espontânea do estado excitado restringe a possibilidade de uma coerência com tempo controlável, ou seja, a superposição se perde de forma aleatória cada vez que há emissão espontânea. A partir essa perspectiva, o sistema de dois níveis não é o candidato mais apropriado para ser usado em uma interface de luz-matéria que armazene informação.

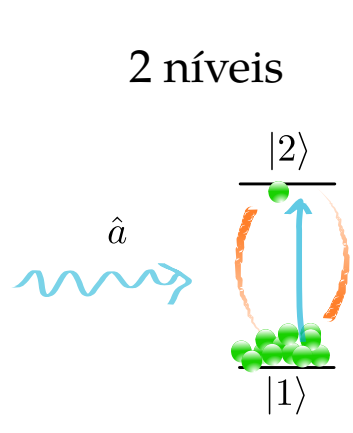

(a)
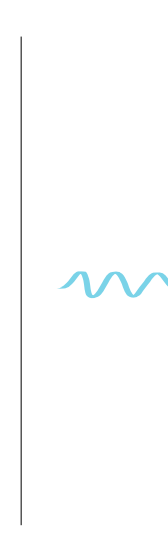

$|1\rangle$

(b)

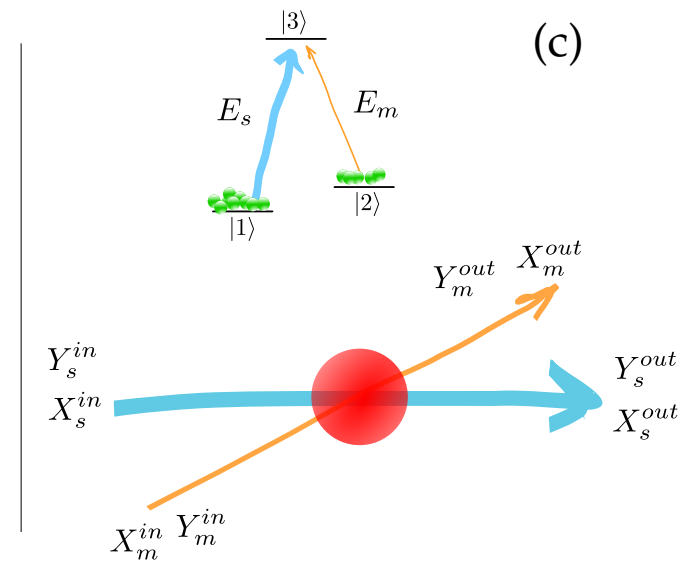

Figura 2.2: (a) Átomo de dois níveis interagindo com um campo â. (b) Átomo de três níveis interagindo com um campo de prova â e um campo de controle $\Omega_{c}$. (c) Implementação de medidas não destrutivas em um sistema de três níveis acoplado por dois campos $E_{s} e E_{m}$.

Consideremos um sistema um pouco mais complexo em estrutura de níveis: o sistema de 
três níveis atômicos como mostra a figura 2.2.(b). Neste sistema temos dois campos óticos acoplados, cada um, às transições $|1\rangle \rightarrow|3\rangle$ e $|2\rangle \rightarrow|3\rangle$. Também consideramos que a transição $|1\rangle \rightarrow|2\rangle$ não é permitida por acoplamento ótico. No entanto, a dinâmica deste sistema permite criar uma superposição entre os estados fundamentais $|1\rangle \leftrightarrow|2\rangle$, mediante técnicas como: as transições Raman dadas pela absorção do campo de prova $\hat{a}$ seguida pela emissão de fótons no campo de controle $\Omega_{c}$, ou a transparência eletromagneticamente induzida (EIT sigla em inglês), que acontece pela interferência destrutiva das excitações óticas $\hat{a}$ e $\Omega_{c}$. No capitulo 3 faremos uma discussão mais detalhada sobre EIT.

Os sistemas de três níveis são então a base para criar interfaces luz-matéria. A partir da perspectiva de estados discretos, a possibilidade de induzir por meios óticos a superposição de estados fundamentais, faz com que seja possível imprimir um estado no meio, ou seja, mapear a informação contida na luz incidente nos estados atômicos de forma controlada [20]. Inclusive, pela baixa perda de coerência entre os estados fundamentais (em particular os estado $|1\rangle$ e $|2\rangle$ da figura 2.2.(b)), é possível ter um controle no tempo de armazenamento de pulsos de luz no meio atômico. Isto é acompanhado de um efeito de diminuição da velocidade de grupo da luz, o que permite prolongar o tempo de interação luz-átomo e, assim fazer do sistema de três níveis um dos principais candidatos para implementar memórias quânticas. Entende-se como memória quântica o dispositivo no qual podemos armazenar estados quânticos da luz. Nela é fundamental a transferência de informação entre luz e átomos, fazendo superposições atômicas, para finalmente recuperar aquilo que foi armazenado de forma controlada no tempo. Tem-se mostrado trabalhos usando transições Raman [63, 64] e alguns outros usando EIT [10, 11, 65] para implementar memórias quânticas.

Assim descrevemos como uma interface mapeia estados de luz em estados atômicos, ambos com carácter discreto. No entanto, agora na perspectiva de estados contínuos, podemos entender essa interface como o meio para fazer medidas quânticas não destrutivas (QND sigla em inglês). Exemplo disso esta na referencia [66], em que um sistema de três níveis é ideal para acoplar um campo sinal $E_{s}$ e um campo de medida $E_{m}$, como mostra a figura 2.2.(c). A ideia nesta interface é medir uma das quadraturas (que é um observável) do campo sinal, dadas por $X_{s}^{i n}$ e $Y_{s}^{i n}$, mediante um campo de medida com quadraturas $X_{s}^{i n}$ 
e $Y_{s}^{\text {in }}$, em que ambos os campos interagem com um sistema de três níveis. O meio permite extrair informação de uma das quadraturas do campo $E_{s}$ mediante a medida nas quadraturas do campo $E_{m}$. Isto é, após a interação com os átomos, as quadraturas $X$ dos campos sinal e de medida permanecem iguais, enquanto que das quadraturas $Y$ permitem extrair parte das quadraturas do sinal $X$, tal que $Y_{s}^{\text {out }}=Y_{s}^{\text {in }}+G X_{m}^{\text {in }}$ e $Y_{m}^{\text {out }}=Y_{m}^{\text {in }}+G X_{s}^{\text {in }}$, onde $G$ é o ganho do sistema. Este seria um tipo de medida não destrutiva entre campos de luz por meio da interação com átomos.

Contudo, o sistema de três níveis não é o único sistema para criar interfaces entre luz e matéria. Podemos estender a estrutura atômica, por exemplo, para um sistema de quatro níveis, ou maior ainda, para um sistema de cinco níveis. O sistema de 4 níveis tem se mostrado como excelente candidato como interface para gerar campos emaranhados [24, 25]. No que diz respeito ao sistema de cinco níveis, há algumas proposta para implementar esse sistema como uma porta lógica de polarização [67]. Aqui trataremos do sistema de cinco níveis unicamente para descrever por completo a dinâmica de estados do sistema estudado na segunda parte do capitulo 3 .

\subsection{Interação luz-matéria}

A seguir descreveremos brevemente o Hamiltoniano de interação entre a radiação e a matéria, que vai nos permitir estudar a dinâmica do sistema de três e cinco níveis no capítulo 3. Seguiremos aqui a descrição para a interação radiação e matéria conforme a referência [49].

\subsubsection{Hamiltoniano mediante interação dipolar}

Consideremos de forma geral um átomo neutro com $k$ níveis inferiores e $k^{\prime}$ níveis excitados, em que temos unicamente acoplamentos óticos entre os níveis inferiores e excitados ${ }^{5}$. O

\footnotetext{
${ }^{5}$ A conotação de "níveis inferiores" referem-se ao conjunto de níveis com energias diferentes, os quais não podem ser acoplados oticamente entre eles, e que possuem uma energia inferior ao níveis denominados como excitados.
} 
hamiltoniano livre dos átomos é dado por

$$
H_{0}=\hbar \sum_{i}^{k} \omega_{(i)}^{g}\left|g_{i}\right\rangle\left\langle g_{i}\left|+\hbar \sum_{i}^{k^{\prime}} \omega_{(i)}^{e}\right| e_{i}\right\rangle\left\langle e_{i}\right|
$$

em que $\hbar \omega_{(i)}^{g}$ e $\hbar \omega_{(i)}^{e}$ representam a energia de ionização dos níveis atômicos inferiores $\left(\left\{\left|g_{i}\right\rangle\right\}\right)$ e excitados $\left(\left\{\left|e_{i}\right\rangle\right\}\right)$, respectivamente.

Por outro lado, consideremos um campo eletromagnético $\mathbf{E}$ como um campo total de $N$ campos eletromagnéticos superpostos. Assim o campo total é escrito como

$$
\mathbf{E}(\mathbf{r}, t)=\sum_{l=1}^{N} \mathbf{E}_{l}(\mathbf{r}, t)
$$

Pela expansão multipolar de uma distribuição de cargas, temos que, segundo $[49,68]$ a interação de um átomo neutro com o campo total $\mathbf{E}$, cujo centro de massa está na posição $\mathbf{r}_{0}$, é determinada pela interação dipolar

$$
H_{\text {int }}=-\mathbf{E}\left(\mathbf{r}_{0}, t\right) \cdot \mathbf{d}
$$

onde o momento dipolar elétrico é representado por $\mathbf{d}=-e \mathbf{r}$ como mostra a figura 2.3 , em que r é a coordenada do elétron com respeito ao centro de massa.

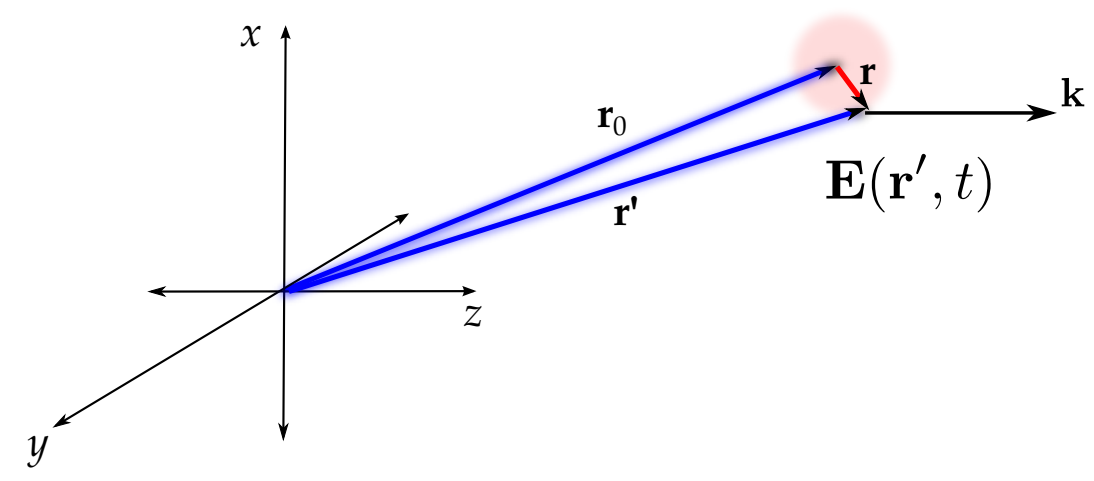

Figura 2.3: Interação átomo- campo.

Para obter este hamiltoniano de interação, se assumiu que o comprimento de onda dos 


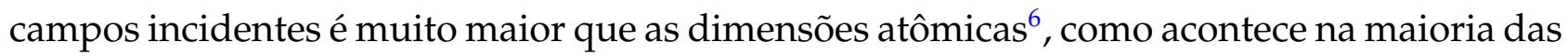
situações experimentais. Deste modo, é valido considerar unicamente as variações temporais do campo, ou seja, $\mathbf{E}(t)$. Uma característica importante deste hamiltoniano de interação é a sua simetria por inversão espacial, isto significa que os estados atômicos devem mudar de paridade quando se transforma $\mathbf{r} \rightarrow-\mathbf{r}$.

A partir dos estados atômicos podemos definir os operadores atômicos $\hat{\sigma}_{g_{i}, g_{j}}=\left|g_{i}\right\rangle\left\langle g_{j}\right|$, $\hat{\sigma}_{e_{i}, e_{j}}=\left|e_{i}\right\rangle\left\langle e_{j}\right|$ e $\hat{\sigma}_{g_{i}, e_{j}}=\left|g_{i}\right\rangle\left\langle e_{j}\right|$ em que $\hat{\sigma}_{g_{i}, g_{i}}$ e $\hat{\sigma}_{e_{i}, e_{i}}$ definem os operadores das populações atômicas. Entretanto, para $i \neq j$ temos todos os operadores das coerências atômicas que podem ser acopladas oticamente $\hat{\sigma}_{g_{i}, e_{j}}$, e as que não são acopladas oticamente $\hat{\sigma}_{g_{i}, g_{j}}$ e $\hat{\sigma}_{e_{i}, e_{j}}$. Estes operadores satisfazem as relações de comutação $\left[\hat{\sigma}_{\alpha \beta}, \hat{\sigma}_{\alpha^{\prime} \beta^{\prime}}\right]=\hat{\sigma}_{\alpha \beta^{\prime}} \delta_{\beta \alpha^{\prime}}-\hat{\sigma}_{\alpha^{\prime} \beta} \delta_{\beta^{\prime} \alpha}$ onde $\alpha=\left\{e_{i}, g_{i}\right\}$ e da mesma forma para $\beta, \alpha^{\prime}$ e $\beta^{\prime}$.

De acordo com o hamiltoniano (2.11), a interação luz-matéria pode ser escrita da forma padrão $[48,49,58,68,69]$, como

$$
H_{\text {int }}=\hbar \sum_{l=1}^{N}\left[\bar{\Omega}_{l} \hat{\sigma}_{e g}^{l} e^{i\left(\mathbf{k}_{l} \cdot \mathbf{r}_{0}-\omega_{l} t+\phi_{l}\right)}+\bar{\Omega}_{l}^{*} \hat{\sigma}_{g e}^{l} e^{-i\left(\mathbf{k}_{l} \cdot \mathbf{r}_{0}-\omega_{l} t+\phi_{l}\right)}\right]
$$

em que pelas definição do campo eletromagnético (1.4a), definimos as frequências de Rabi como $\Omega_{i}=-\mathcal{E}_{i}\left(\mathbf{e}_{i} \cdot \mathbf{d}_{g_{i} e_{j}}\right) / \hbar$. O vectores $\mathbf{d}_{g_{i} e_{j}}=\left\langle g_{i}|\hat{\mathbf{d}}| e_{j}\right\rangle$ correspondem ao valor esperado do momento dipolar atômico, que determina as regras de seleção para uma dada transição atômica. Os operadores $\hat{\sigma}_{e g}^{l}=\left[\hat{\sigma}_{e_{i}, g_{j}}\right]_{l}$ representam os operadores de transição entre os níveis $\left|g_{i}\right\rangle$ e $\left|e_{j}\right\rangle$ associados univocamente ao acoplamento do campo l-ésimo. Para cada sistema que trataremos aqui, serão especificados os campos que acoplam cada uma dessas coerências. A fase $\phi_{l}$ é levada em conta para uma descrição geral dos campos, e que pode conter flutuações intrínsecas da fonte ou defasagens $k \Delta z$ induzidas por pequenos deslocamentos $\Delta z$ dos átomos.

Esta forma de abordar o hamiltoniano, em termos dos níveis $\left|g_{i}\right\rangle$ e $\left|e_{j}\right\rangle$ arbitrários, permite descrever qualquer estrutura de níveis atômicos, dependendo do sistema a ser estu-

\footnotetext{
${ }^{6}$ Isto é $2 \pi a_{0} / \lambda \ll 1$ onde $\lambda$ é o comprimento de onda da luz e $a_{0}$ é o rádio de Bohr.
} 
dado. Por exemplo, se temos estados hiperfinos que apresentam degenerescência em energia, os estados $\left|g_{i}\right\rangle$ e $\left|e_{j}\right\rangle$ são associados aos estados Zeeman, em que as polarizações linear ou circular do vetor $\mathbf{e}_{i}$ definem diferentes tipos de transições $\mathbf{d}_{g_{i} e_{j}}$ entre os níveis Zeeman. Entretanto, se ignoramos qualquer tipo de degenerescência, os estados $\left|g_{i}\right\rangle$ e $\left|e_{j}\right\rangle$ podem representar simplesmente os estados hiperfinos, em que a polarização $\mathbf{e}_{i}$ define um momento dipolar efetivo $\mathbf{d}_{g_{i} e_{j}}$. Chamamos a atenção nesse ponto, já que lidaremos com níveis Zeeman para tratar o efeito de EIT na primeira parte do capítulo 3 e consideramos níveis hiperfinos assumindo momento dipolar efetivo para um sistema de 5 níveis na última parte do capítulo 3.

A seguir, analisaremos um caso particular do hamiltoniano (2.12): o sistema de três níveis. Algumas propriedades deste sistema serão amplamente estudadas ao longo desta tese.

\section{Sistema de três níveis}

Consideremos um sistema atômico de três níveis (3N) com auto-estados $|1\rangle$, $|2\rangle$ e $|3\rangle$ do hamiltoniano livre e que interagem com dois campos óticos (clássicos) em configuração $\Lambda$ como é mostrado na figura 2.2.(b). $\mathrm{O}$ campo $\mathbf{E}_{1}$ com frequência $\omega_{1}$ acopla a transição entre o estado $|1\rangle$ e $|3\rangle$ e o campo $\mathbf{E}_{\mathbf{2}}$ com frequência $\omega_{2}$ acopla a transição entre os estados $|2\rangle$ e $|3\rangle$. Portanto o hamiltoniano total do sistema é dado por

$$
H^{3 N}=H_{o}^{3 N}+H_{\text {int }}^{3 N},
$$

onde $H_{o}$ é o hamiltoniano dos átomos em condição livre e $H_{\text {int }}$ é o hamiltoniano de interação dos átomos com os campos eletromagnéticos. De acordo com o hamiltoniano livre (2.9) reduzido para três níveis, ele pode ser rescrito como

$$
H_{o}^{3 N}=\sum_{j=1}^{3} \hbar \omega_{(j)} \hat{\sigma}_{j} ; \quad \hat{\sigma}_{j}=|j\rangle\langle j|
$$


sendo $\omega_{(j)}$ a frequência de transição de cada nível atômico em relação ao nível de energia de ionização e $\hat{\sigma}_{j}$ corresponde à população atômica de cada nível. e da expressão geral para o hamiltoniano de interação (2.12), temos

$$
H_{\text {int }}^{3 N}=\hbar\left(\Omega_{1}^{*} \hat{\sigma}_{13} e^{i\left(\omega_{1} t+\varphi_{1}\right)}+\Omega_{2}^{*} \hat{\sigma}_{23} e^{i\left(\omega_{2} t+\varphi_{2}\right)}+\text { adj. }\right),
$$

onde $\varphi_{l}=-\left(\phi_{l}+\mathbf{k}_{l} \cdot \mathbf{r}_{0}\right)$ sendo $l=1,2$.

\subsubsection{Dinâmica do sistema átomo-campo: equações de Bloch}

Dado o hamiltoniano do sistema átomo-luz, a dinâmica do sistema é dada pelas equações de Heisenberg-Langevin para as variáveis atômicas tal que

$$
\frac{d \hat{\sigma}_{i j}(t)}{d t}=-\frac{i}{\hbar}\left[\hat{\sigma}_{i j}(t), H(t)\right]+\Gamma_{i j} \hat{\sigma}_{i j}(t)+\hat{F}_{i}(t)
$$

em que $\Gamma_{i j}$ corresponde à matriz de decaimento e os operadores $\hat{F}_{i}(t)$ representam os operadores de flutuações que possuem características estocásticas, como descrevemos na seção 1.3 , de modo que o seu valor médio é $\left\langle\hat{F}_{i}(t)\right\rangle=0$. Estas flutuações representam o acoplamento do sistema com um reservatório, ou seja, consideramos um sistema aberto que está em contanto com fontes que podem gerar excitações nas variáveis atômicas de forma aleatória. Essa fonte podem ser os modos do campo eletromagnético no estado de vácuo, os quais se tornam canais de emissão para o átomo decair de um estado excitado para o estado fundamental. É devido ao acoplamento com reservatório que se mostra a existência de emissão espontânea nos átomos. Portanto, o primeiro termo do lado direito da expressão (2.16) é responsável pela dinâmica reversível do sistema, enquanto o segundo termo contribui para a sua dinâmica irreversível (típico dos sistemas com dissipação ou perda de energia).

De acordo com o caso tratado veremos especificamente, para cada um, a equação dinâmica dos operadores do sistema. Além disso, dado que os operadores atômicos estão relacionados com os elementos da matriz densidade mediante o valor médio $\rho_{i j}(t)=\left\langle\hat{\sigma}_{i j}(t)\right\rangle$, podemos 
obter de forma direta a evolução temporal para as populações e coerências atômicas, tal que

$$
\frac{d \rho_{i j}(t)}{d t}=-\frac{i}{\hbar}\left\langle\left[\hat{\sigma}_{i j}(t), H(t)\right]\right\rangle+\Gamma_{i j} \rho_{i j}(t)
$$

em que o termo do operador de flutuação se cancela, devido a $\left\langle F_{i}(t)\right\rangle=0$. O conjunto de equações que surge a partir desta expressão definem as equações de Bloch. Esta dinâmica também pode ser obtida mediante a equação mestra, como é abordado nas referências $[7,49$, 58]. No entanto, neste trabalho adotaremos o tratamento de Heisenberg-Langevin, em que, usaremos a eq. (2.17) para determinar a dinâmica das variáveis atômicas de acordo com o caso.

Apresentamos assim uma breve introdução da evolução temporal para um sistema atômico com uma estrutura de níveis arbitrários interagindo com campos clássicos e quânticos. Veremos a seguir como essa interação produz forças nos átomos, gerando assim um acoplamento entre seus graus de liberdade internos e externos.

\subsection{Força devido à interação luz-átomos}

As primeiras conjeturas sobre a possibilidade da luz exercer força na matéria vem do século XVII, quando Keppler observou que a cauda dos comentas sempre apontam em direção oposta ao sol. Mais tarde, a electrodinâmica de Maxwell permitiu realizar descrição formal sobre a força que a luz exerce sobre a matéria. No entanto, só no começo do seculo XX foram feitas experiências que mostravam quantitativamente o efeito da pressão de radiação na matéria. Segundo W. Phillips ${ }^{7}$ [70], a primeira demonstração experimental da pressão de radiação nos átomos só se deu em 1933 por Frisch, ao desviar um feixe de átomos mediante uma lâmpada . Posteriormente Ashkin reconheceu o grande potencial do laser para manipular átomos, fazendo experiências similares às de Frisch em 1972. A partir desse momento, a comunidade de física atômica, impressionada e motivada pela possibilidade de manipular átomos à vontade, colocou muito do seu interesse em resfriar e armadilhar íons e posterior-

\footnotetext{
${ }^{7}$ quem ganhou premio nobel em 1997 junto com Steven Chu e Cohen-Tannoudji, pela sua contribuição ao resfriamento e aprisionamento de átomos neutros com lasers
} 
mente átomos neutros.

Nesta seção faremos uma revisão do modelo semiclássico que descreve a força que a luz exerce nos átomos. Seguiremos aqui a abordagem apresentada em [68]. Trata-se de uma revisão importante, não somente para entender a preparação dos átomos frios no nosso experimento, mas também para explicar alguns efeitos dos resultados apresentados no capitulo 4.

\subsubsection{Força em um sistema de 2 níveis}

Consideremos um átomo de dois níveis interagindo com luz de frequência $\omega_{L}$ e propagandose em direção k. De acordo com a eq.(2.12), o hamiltoniano de interação para este sistema é dado por

$$
H_{\mathrm{int}}^{2 N}=\hbar\left(\Omega_{L}\left(\mathbf{r}^{\prime}\right) \hat{\sigma}_{e g} e^{i\left(\mathbf{k} \cdot \mathbf{r}^{\prime}-\omega_{L} t\right)}+\text { adj. }\right)
$$

em que $\sigma_{e g}=|e\rangle\langle g|$ e aqui consideramos $\mathbf{r}^{\prime}=\mathbf{r}_{0}+\mathbf{r}$, já que vamos analisar o caso geral em que o campo apresenta variações de amplitude ao longo da direção de propagação. A frequência de Rabi $\Omega_{L}\left(\mathbf{r}^{\prime}\right)$ é o caso geral em que o campo apresenta um perfil de modo espacial ${ }^{8}$. Então, a força que a luz exerce em um átomo, segundo o teorema de Ehrenfest, é dada pelo valor esperado

$$
\overline{\mathbf{F}}=\langle\mathbf{F}\rangle=\frac{d}{d t}\langle\mathbf{p}\rangle=-\langle\nabla V\rangle
$$

onde $\mathbf{F}$ representa o operador de força, $\mathbf{p}$ o operador de momento e $V$ é a energia potencial do sistema, que neste caso é dada pelo hamiltoniano de interação $H_{\text {int }}^{2 N}$ da eq.(2.18). Desta forma, temos que a força é

$$
\overline{\mathbf{F}}=-\hbar\left\langle\nabla\left[\Omega_{L}\left(\mathbf{r}^{\prime}\right) e^{i \mathbf{k} \cdot \mathbf{r}^{\prime}}\right] \hat{\sigma}_{e g} e^{-i \omega_{L} t}+\operatorname{adj} .\right\rangle
$$

\footnotetext{
${ }^{8}$ Por exemplo, o átomo pode interagir com luz que apresenta um perfil de intensidade gaussiano.
} 
onde é importante notar que $\nabla$ é o gradiente com relação à variável $\mathbf{r}$. Ao fazermos a expansão de $\Omega_{L}\left(\mathbf{r}^{\prime}\right) e^{i \mathbf{k} \cdot \mathbf{r}^{\prime}}$ em torno de $\mathbf{r}_{0}$, temos que

$$
\begin{aligned}
\Omega_{L}\left(\mathbf{r}^{\prime}\right) e^{i \mathbf{k} \cdot \mathbf{r}^{\prime}} & =\Omega_{L}\left(\mathbf{r}_{0}\right) e^{i \mathbf{k} \cdot \mathbf{r}_{0}}+\left.\left(\mathbf{r}^{\prime}-\mathbf{r}_{0}\right) \cdot \nabla^{\prime}\left[\Omega_{L}\left(\mathbf{r}^{\prime}\right) e^{i \mathbf{k} \cdot \mathbf{r}^{\prime}}\right]\right|_{\mathbf{r}_{0}}+\cdots \\
& =\Omega_{L}\left(\mathbf{r}_{0}\right) e^{i \mathbf{k} \cdot \mathbf{r}_{0}}+\left(\mathbf{r}^{\prime}-\mathbf{r}_{0}\right) \cdot\left[\left.\nabla^{\prime} \Omega_{L}\left(\mathbf{r}^{\prime}\right)\right|_{\mathbf{r}_{0}}+i \mathbf{k} \Omega_{L}\left(\mathbf{r}_{0}\right)\right] e^{i \mathbf{k} \cdot \mathbf{r}_{0}}+\cdots
\end{aligned}
$$

onde o gradiente $\nabla^{\prime}$ está relacionado com a variável $\mathbf{r}^{\prime}$. Por definição temos que $\mathbf{r}^{\prime}-\mathbf{r}_{0}=$ r, e ao assumir a aproximação dipolar, consideramos então a expansão da eq.(2.21) até termos de primeira ordem em $\mathbf{r}$. Portanto, o gradiente da eq.(2.20) é $\nabla\left[\Omega_{L}\left(\mathbf{r}^{\prime}\right) e^{i \mathbf{k} \cdot \mathbf{r}^{\prime}}\right]=$ $\left[\left.\nabla^{\prime} \Omega_{L}\left(\mathbf{r}^{\prime}\right)\right|_{\mathbf{r}_{0}}+i \mathbf{k} \Omega_{L}\left(\mathbf{r}_{0}\right)\right] e^{i \mathbf{k} \cdot \mathbf{r}_{0}}$ e assim a força é escrita como

$$
\overline{\mathbf{F}}=-\hbar\left[\left.\nabla^{\prime} \Omega_{L}\left(\mathbf{r}^{\prime}\right)\right|_{\mathbf{r}_{0}}+i \mathbf{k} \Omega_{L}\left(\mathbf{r}_{0}\right)\right]\left\langle\hat{\sigma}_{e g}\right\rangle e^{-\left(i \omega_{L} t+\phi\right)}+\operatorname{adj} .
$$

onde podemos notar que o primeiro termo da força é proporcional ao gradiente $\left.\nabla \Omega_{L}\left(\mathbf{r}^{\prime}\right)\right|_{\mathbf{r}_{0}}$ do campo na posição do átomo, e o segundo termo é proporcional à intensidade $\Omega_{L}\left(\mathbf{r}_{0}\right)$ em direção de propagação do campo. Isto vai definir certas características para cada tipo de força, que estará determinada pela coerência entre os estados acoplados, sendo $\left\langle\hat{\sigma}_{e g}\right\rangle=\rho_{e g}$. Mais adiante veremos que a resposta atômica lentamente variável está contida no termo $\tilde{\rho}_{e g}=\rho_{e g} e^{-i\left(\omega_{L} t+\phi\right)}$, de modo que a força que a luz exerce nos átomos é

$$
\overline{\mathbf{F}}=-\underbrace{\left.2 \hbar \nabla^{\prime} \Omega_{L}\left(\mathbf{r}^{\prime}\right)\right|_{\mathbf{r}_{0}} \operatorname{Re} \tilde{\rho}_{g e}}_{\text {F. Dipolo }}+\underbrace{2 \hbar \mathbf{k} \Omega_{L}\left(\mathbf{r}_{0}\right) \operatorname{Im} \tilde{\rho}_{g e}}_{\text {F. Espalhamento }}
$$

A primeira componente corresponde à força de dipolo, na qual o gradiente do campo na região do átomo pode gerar forças para aprisioná-lo ou repeli-lo radialmente em torno do eixo de propagação. O aprisionamento ou repulsão do átomo será determinado unicamente pela dispersão do meio representada por $\operatorname{Re} \tilde{\rho}_{g e}$ e que toma valores positivos ou negativos segundo a dessintonia da luz com relação à ressonância atômica. Entretanto, o segundo termo corresponde à força de espalhamento, que é exercida na mesma direção da propagação do campo e é determinada unicamente pela absorção do meio dada por $\operatorname{Im} \tilde{\rho}_{g e}$, como mostra a 
figura 2.4 (a). Em outras palavras, essa é a força que gera a pressão de radiação nos átomos.

(a)

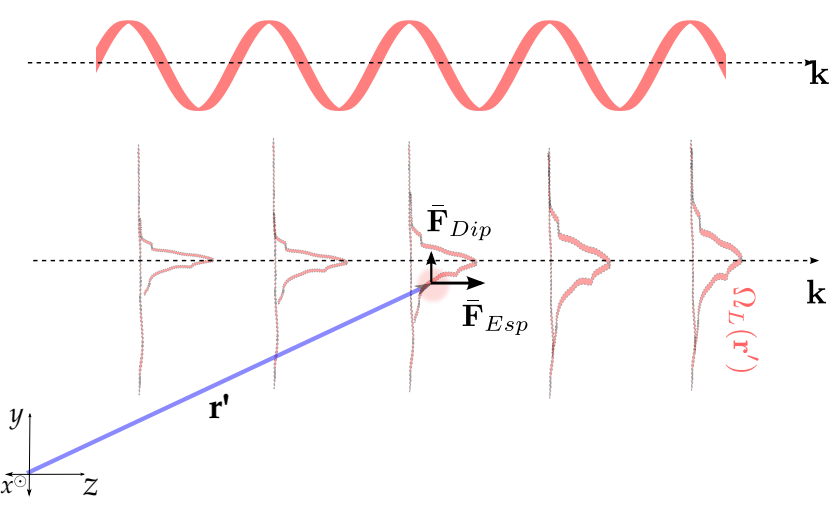

(b)

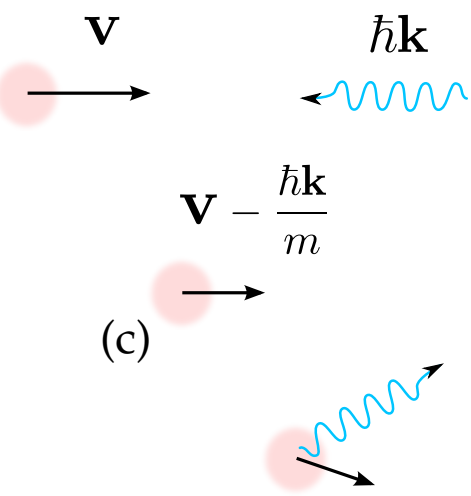

(d)

Figura 2.4: (a)Força exercida por um campo com perfil $\Omega_{L}(\mathbf{r})$, que se descompõe em duas forças: de dipolo e de espalhamento. (b) Um átomo com velocidade $\mathbf{b}$ que vai interagir com um fóton em direção oposta e momento $\hbar|\mathbf{k}|$. (c) Depois de absorver o fóton o átomo se desloca no espaço com uma velocidade menor $a \mathbf{v}-\hbar \mathbf{k} / m$. (d) Depois de emitir espontaneamente um fóton em direção aleatória, a força de recuo é nula, e portanto, a velocidade do átomo é reduzida com relação ao caso (a).

No caso em que temos um feixe colimado e quase homogêneo, então $\left.\nabla^{\prime} \Omega\left(\mathbf{r}^{\prime}\right)\right|_{\mathbf{r}_{0}} \approx 0$ e portanto a força resultante exercida nos átomos é principalmente a de espalhamento, tal que $\overline{\mathbf{F}} \approx 2 \hbar \mathbf{k} \Omega_{L}\left(\mathbf{r}_{0}\right) \operatorname{Im} \tilde{\rho}_{g e}$.

A força de espalhamento que a luz exerce nos átomos é explorada pelas mais diversas técnicas para reduzir a velocidade de átomos neutros. De forma muito simples podemos entender o seu princípio. Consideremos um átomo com velocidade $\mathbf{v}$ que interage com um fóton com momento $-\hbar \mathbf{k}$, como mostra a figura 2.4 (b). Ao absorver o fóton, o átomo tem sua velocidade reduzida pela troca de momento, como é representado na figura (c). Após a absorção do fóton, o átomo pode emitir luz de forma espontânea em direções aleatórias, como é representado na figura (d). O efeito da força de recuo associada a essa emissão espontânea é nulo, pois a luz é emitida aleatoriamente em todas as direções, cancelando seu efeito no valor médio das forças. Portanto, a velocidade inicial $\mathbf{v}$ do átomo é reduzida para um valor $\mathbf{v}-\hbar \mathbf{k} / m$.

No capitulo 4 descreveremos brevemente o que é uma armadilha magneto-óptica, com a qual preparamos os átomos do nosso experimento, e que se baseia na força de espalhamento. 
A descrição das forças aqui apresentadas serão fundamentais para entender também alguns dos seus efeitos no caso em que considerarmos um átomo de três e cinco níveis. A força da luz nos átomos vai depender do hamiltoniano de interação, como foi mostrado na eq.(2.19).

\section{Conclusões do capítulo}

Ao longo deste capítulo analisamos a interface entre a luz e a matéria a partir de uma perspectiva macroscópica e microscópica. Na descrição macroscópica, revisamos as propriedades lineares de polarização de um meio atômico em resposta à interação com a luz. Na descrição microscópica, revisamos a interação átomo-luz descrita pelo hamiltoniano de interação de forma geral para átomos com $N$ níveis de energia. Em particular, revisamos o hamiltoniano para meios com estrutura atômica de três, que será a base para os próximos capítulos. Por último, fizemos uma revisão da descrição semiclássica dos tipos de forças que a luz pode exercer nos átomos. Veremos como o nosso experimento é sensível a esse tipo de força. 


\section{Parte II}

\section{Espectroscopia de correlação em}

\section{transparência eletromagneticamente induzida}





\section{CAPÍTULO 3 \\ ASPECTOS TEÓRICOS DAS FLUTUAÇÕES DA LUZ EM \\ CONDIÇÃO DE TRANSPARÊNCIA \\ ELETROMAGNETICAMENTE INDUZIDA}

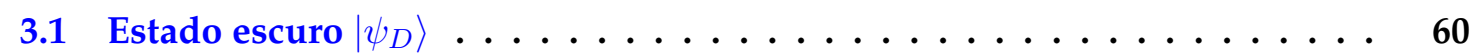

3.2 Susceptibilidade dielétrica linear: condição de EIT . . . . . . . . . . . 62

3.3 Transformação de ruído de fase para ruído de amplitude (RF-RA) . . . . 67

3.4 Modelo heurístico para correlação $g^{(2)}(0)$ para EIT $\ldots \ldots \ldots \ldots$

3.5 Dinâmica do sistema átomo-campo com fase estocástica mediante cálculo

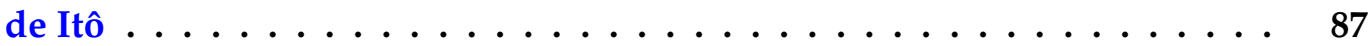

3.6 Resultados para a dinâmica átomo-campo mediante o método perturbativo 103

Neste capítulo abordaremos como as flutuações de amplitude e fase da luz são alteradas pela interação com átomos em uma interface de transparência eletromagneticamente induzida (EIT). Começaremos então analisando as condições necessárias para que a transparência induzida aconteça em um átomo de três níveis e descreveremos as suas principais características. Em seguida, analisaremos como as propriedades estatísticas da luz são 
modificadas pelos átomos em condição de EIT. Faremos uma breve revisão dos principais trabalhos que se aprofundaram no estudo deste tipo de sistema.

Depois, revisaremos a abordagem no domínio temporal e das frequências proposta por nós no LMCAL (sigla para Laboratório de manipulação coerente de átomos e luz). Em particular, revisaremos o modelo heurístico para a correlação no domínio no tempo e o modelo de fase estocástica para determinar a correlação no domínio das frequências.

Na segunda metade deste capítulo, apresentamos um dos primeiros resultados deste trabalho. Apresentaremos o tratamento perturbativo para a dinâmica de Langevin das variáveis atômicas. Veremos como a correlação se descompõe nos diferentes termos perturbativos. Mostraremos as principais vantagens deste modelo com respeito aos modelos propostos até hoje. Esta proposta nos permitirá entender algumas das características observadas nos resultados experimentais descritos no capítulo 4 .

\subsection{Estado escuro $\left|\psi_{D}\right\rangle$}

Consideremos o hamiltoniano do sistema de três níveis a partir do hamiltoniano de interação (2.12). Nele, consideramos dois campos $\mathbf{E}_{1}$ e $\mathbf{E}_{2}$ em configuração $\Lambda$, acoplando as duas transições atômicas, com dessintonias $\Delta_{1}$ e $\Delta_{2}$ com relação ao estado excitado, como mostra a figura 3.1.(a).

De acordo com a seção 2.3.1 o hamiltoniano livre do sistema de três níveis é dado por $H_{o}^{3 N}=\sum_{j=1}^{3} \hbar \omega_{(j)} \hat{\sigma}_{j}$. Aplicando a transformação unitária $U=e^{-i H_{o} t \hbar}$ ao hamiltoniano de interação da expressão (2.15), temos

$$
H_{\mathrm{int}}^{\prime}=e^{\frac{i H_{o} t}{\hbar}} H_{\mathrm{int}} e^{-\frac{i H_{o} t}{\hbar}}=\hbar\left[\Omega_{1}^{*} \hat{\sigma}_{13} e^{i\left(\Delta_{1} t+\varphi_{1}\right)}+\Omega_{2}^{*} \hat{\sigma}_{23} e^{i\left(\Delta_{2} t+\varphi_{2}\right)}+H . c .\right]
$$

em que $\Delta_{i}=\omega_{i}-\left(\omega_{(3)}-\omega_{(i)}\right)$ sendo $i=1$, 2. Em particular, podemos analisar o caso em que os campos estão em ressonância com as transições atômicas, ou seja $\Delta_{1}=\Delta_{2}=0$, como mostra a figura 3.1.(b). 


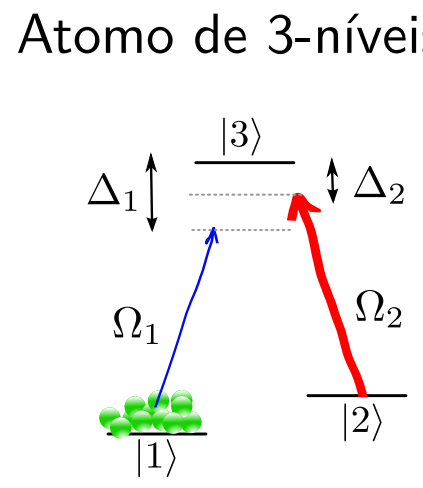

(a)

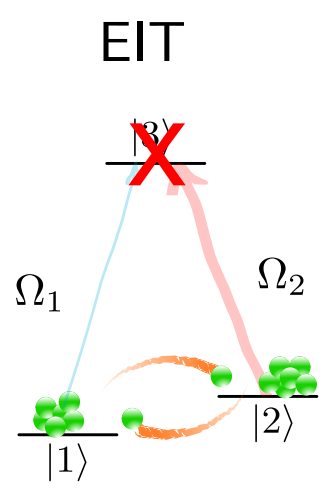

(b)

Figura 3.1: (a) Sistema de três níveis interagindo com um campo de prova e um campo de controle, em uma configuração $\Lambda$. (b) Estado escuro $\left|\psi_{D}\right\rangle$ da eq.(3.3).

O objetivo é achar os autoestados deste hamiltoniano e determinar quais deles estão relacionados com o fenômeno de transparência. A representação matricial do hamiltoniano de interação na base de estados $\{|1\rangle,|2\rangle,|3\rangle\}$ é dada pelos elementos $\left\langle i\left|H_{\text {int }}^{\prime}\right| j\right\rangle$, de modo que

$$
H_{\mathrm{int}}^{\prime}=\hbar\left(\begin{array}{ccc}
0 & 0 & \Omega_{1} e^{-i \varphi_{1}} \\
0 & 0 & \Omega_{2} e^{-i \varphi_{2}} \\
\Omega_{1} e^{i \varphi_{1}} & \Omega_{2} e^{i \varphi_{2}} & 0
\end{array}\right)
$$

Ao diagonalizar esta matriz obtemos os autoestados do hamiltoniano. São eles:

- O Estado Escuro com autovalor $\lambda_{D}=0$

$$
\left|\psi_{D}\right\rangle=\cos \theta e^{-i \varphi_{1}}|1\rangle-\sin \theta e^{-i \varphi_{2}}|2\rangle
$$

em que $\tan \theta=\Omega_{1} / \Omega_{2}$. Este estado é a superposição dos estados fundamentais $|1\rangle \mathrm{e}$ $|2\rangle$. Ela depende das frequências de Rabi e das fases relativas entre o campo de prova e de controle. Independente do valor desses parâmetros, a probabilidade de achar o sistema no estado excitado $|3\rangle$ é nula, ou seja, $\left\langle 3 \mid \psi_{D}\right\rangle=0$ e, portanto, o meio se torna transparente à luz. Isto se dá devido à interferência destrutiva entre as excitações associadas aos campos de prova e controle, que desacoplam o estado excitado $|3\rangle$. Aliás, 
no estado escuro não só os campos não acoplam o nível excitado, como também, devido ao fato do autovalor associado ser nulo $(\lambda=0)$, o átomo é aprisionado tal que a evolução temporal é

$$
\left|\psi_{I}(t)\right\rangle=e^{\frac{i H_{\text {int }}^{\prime}}{\hbar}}\left|\psi_{D}\right\rangle=\left|\psi_{D}\right\rangle
$$

Isto é conhecido como aprisionamento coerente de população (CPT- Coherent Population Trapping). Inclusive, se considerarmos um estado inicial arbitrário e levarmos em conta a taxa de decaimento do estado excitado, enquanto a condição Raman for satisfeita, isto é, $\delta=\Delta_{1}-\Delta_{2}=0$, os campos evoluirão para o estado escuro e, portanto, teremos transparência.

- Estados $|\psi \pm\rangle$ : são os estados com autovalores $\lambda_{ \pm}= \pm \hbar \Omega$ sendo $\Omega=\sqrt{\left|\Omega_{1}\right|^{2}+\left|\Omega_{2}\right|^{2}}$, dados por

$$
\left|\psi_{ \pm}\right\rangle=\frac{1}{\sqrt{2}}\left(|3\rangle \pm\left|\psi_{A}\right\rangle\right)
$$

onde $\left|\psi_{A}\right\rangle=\sin \theta e^{i \varphi_{1}}|1\rangle+\cos \theta e^{i \varphi_{2}}|2\rangle$ definido como o estado de acoplamento. Esses estados, à diferença do estado escuro, mantém o acoplamento do estado excitado, tal que $\left\langle 3 \mid \psi_{ \pm}\right\rangle=1 / \sqrt{2}$.

\subsection{Susceptibilidade dielétrica linear: condição de EIT}

Consideremos agora a condição geral em que a dessintonia $\Delta_{1}$ e $\Delta_{2}$ dos campos $\mathbf{E}_{1}$ e $\mathbf{E}_{2}$, respectivamente, podem tomar valores arbitrários. Ao estudar a resposta ótica linear para este tipo de sistemas, as principais propriedades a serem caracterizadas são a absorção e a dispersão dos campos. Elas estão diretamente relacionadas com a susceptibilidade linear, como foi revisado na seção 2.1. De acordo com a eq.(2.3), a polarização devida aos campos de prova e de controle é dada por

$$
\mathbf{P}_{i}(\mathbf{r}, t)=\epsilon_{0} \chi_{i}^{(1)} \mathbf{E}_{i}(\mathbf{r}, t)=\bar{n} \mathbf{d}_{i 3} \rho_{i 3}
$$


em que $\bar{n}$ é a densidade atômica e $\chi_{i}^{(1)}$ representa a susceptibilidade linear associada a cada campo $i=1,2$. Dada a relação entre as polarizações $\mathbf{P}_{1}(\mathbf{r}, t)$ e $\mathbf{P}_{2}(\mathbf{r}, t)$ com os elementos da matriz densidade $\rho_{13}$ e $\rho_{23}$, é necessário determinar as suas soluções estacionárias e, assim, obter uma expressão para a susceptibilidade linear associada ao campo de prova.

A partir do hamiltoniano de interação (2.15) e de acordo com a eq.(2.17), as equações de Bloch para este sistema de três níveis são

$$
\begin{aligned}
& \dot{\tilde{\rho}}_{11}=i \Omega_{1}\left(\tilde{\rho}_{13}-\tilde{\rho}_{31}\right)+\Gamma \tilde{\rho}_{33} \\
& \dot{\tilde{\rho}}_{22}=i \Omega_{2}\left(\tilde{\rho}_{23}-\tilde{\rho}_{32}\right)+\Gamma \tilde{\rho}_{33} \\
& \dot{\tilde{\rho}}_{33}=-i \Omega_{1}\left(\tilde{\rho}_{13}-\tilde{\rho}_{31}\right)-i \Omega_{2}\left(\tilde{\rho}_{23}-\tilde{\rho}_{32}\right)-2 \Gamma \tilde{\rho}_{33} \\
& \dot{\tilde{\rho}}_{13}=-\left(\Gamma-i \Delta_{1}\right) \tilde{\rho}_{13}-i \Omega_{1}\left(\tilde{\rho}_{33}-\tilde{\rho}_{11}\right)-i \Omega_{2} \tilde{\rho}_{12} \\
& \dot{\tilde{\rho}}_{32}=-\left(\Gamma+i \Delta_{2}\right) \tilde{\rho}_{32}-i \Omega_{2}^{*}\left(\tilde{\rho}_{33}-\tilde{\rho}_{22}\right)+i \Omega_{1}^{*} \tilde{\rho}_{12} \\
& \dot{\tilde{\rho}}_{12}=-\left(\gamma_{d}-i \delta\right) \tilde{\rho}_{12}-i \Omega_{1} \tilde{\rho}_{32}-i \Omega_{2}^{*} \tilde{\rho}_{13}
\end{aligned}
$$

em que temos usado a notação $\tilde{\rho}_{i 3}=\rho_{i 3} e^{i\left(\omega_{i} t+\phi_{i}\right)}$ e $\tilde{\rho}_{12}=\rho_{12} e^{i\left(\left(\omega_{1}-\omega_{2}\right) t+\phi_{1}-\phi_{2}\right)}$. Além disso, $\delta=\Delta_{1}-\Delta_{2}$ representa a dessintonia de dois fótons, $\Gamma$ a taxa de emissão espontânea e $\gamma_{d}$ modela os mecanismos de decoerência ${ }^{1}$ entre os estados fundamentais, ou seja, o tempo $1 / \gamma_{d}$ que o sistema leva para perder a superposição do estados fundamentais $|1\rangle$ e $|2\rangle$. Em particular, vamos considerar a situação em que temos um campo muito intenso de bombeio e um campo de prova com intensidade muito menor, tal que $\Omega_{2} \gg \Omega_{1}$. A partir dessa relação de intensidades podemos então abordar a dinâmica do sistema de forma perturbativa [65]. Se o átomo interagisse unicamente com o campo de bombeio, o estado atômico evoluiria para um estado em que $\tilde{\rho}_{11}=1$ e $\tilde{\rho}_{22}=\tilde{\rho}_{33}=0$. A partir deste estado podemos aproximar a

\footnotetext{
${ }^{1}$ Alguns desses mecanismos de decoerência são devido às colisões entre átomos, às colisões entre os átomos e as paredes da câmara (no caso de átomos numa célula de vapor) e à interação dos átomos com campos magnéticos. No caso de átomos frios, consideramos unicamente a decoerência devido à colisões entre os átomos e interação com possíveis campos residuais.
} 
evolução das variáveis atômicas das eqs.(3.10) e (3.11) como

$$
\begin{aligned}
& \dot{\tilde{\rho}}_{13} \approx-\left(\Gamma-i \Delta_{1}\right) \tilde{\rho}_{13}+i \Omega_{1}-i \Omega_{2} \tilde{\rho}_{12} \\
& \dot{\tilde{\rho}}_{32} \approx-\left(\Gamma+i \Delta_{2}\right) \tilde{\rho}_{32}+i \Omega_{1}^{*} \tilde{\rho}_{12},
\end{aligned}
$$

Em condição estacionária, consideramos $\frac{d \rho_{i j}}{d t}=0$ e, portanto, obtemos um sistema linear para as coerências atômicas, de modo que

$$
\begin{aligned}
& \tilde{\rho}_{13}=-i \frac{\Omega_{1}}{\left(\Gamma-i \Delta_{1}\right)}-i \frac{\Omega_{2}}{\left(\Gamma-i \Delta_{1}\right)} \tilde{\rho}_{12}, \\
& \tilde{\rho}_{32}=i \frac{\Omega_{1}^{*}}{\left(\Gamma+i \Delta_{2}\right)} \tilde{\rho}_{12}, \\
& \tilde{\rho}_{12}=-\left(\gamma_{d}-i \delta\right) \tilde{\rho}_{12}-i \Omega_{1} \tilde{\rho}_{32}-i \Omega_{2}^{*} \tilde{\rho}_{13},
\end{aligned}
$$

Assim, ao resolvermos o sistema de equações, obtemos a solução para a coerência $\rho_{13}$ associada à resposta da polarização com respeito ao campo de prova, tal que

$$
\tilde{\rho}_{13}=i\left[\frac{\left(i \delta+\gamma_{d}\right)\left(i \Delta_{2}+\Gamma\right)+\Omega_{1}^{2}}{\left(i \Delta_{1}+\Gamma\right)\left(i \delta+\gamma_{d}\right)\left(i \Delta_{2}+\Gamma\right)+\Omega_{2}^{2}\left(i \Delta_{2}+\Gamma\right)}\right] \Omega_{1} .
$$

de modo que à susceptibilidade a primeira ordem no campo de prova, segundo a eq.(3.6), é dada por $\chi^{(1)}=\bar{n} d_{13} \tilde{\rho}_{13} /\left(\epsilon_{0} E_{1}\right)$. Portanto, de acordo com a eq.(3.18) e lembrando que $\Omega_{1}=d_{13} E_{1} / \hbar$, a dispersão e a absorção dos campos são dadas por

$$
\begin{aligned}
& \operatorname{Re}\left\{\chi^{(1)}\right\}=\frac{\bar{n}\left|d_{13}\right|^{2}}{\epsilon_{o} \hbar}\left\{\frac{\Delta_{p}\left(\gamma_{d}^{2}+\delta^{2}\right)-\Omega_{c}^{2} \delta}{\left|\left(i \Delta_{p}+\Gamma\right)\left(i \delta+\gamma_{d}\right)+\Omega_{c}^{2}\right|^{2}}\right\} \\
& \operatorname{Im}\left\{\chi^{(1)}\right\}=\frac{\bar{n}\left|d_{13}\right|^{2}}{\epsilon_{o} \hbar}\left\{\frac{\gamma_{d} \Omega_{c}^{2}+\Gamma\left(\gamma_{d}^{2}+\Delta_{c}^{2}\right)}{\left|\left(i \Delta_{p}+\Gamma\right)\left(i \delta+\gamma_{d}\right)+\Omega_{c}^{2}\right|^{2}}\right\}
\end{aligned}
$$

Se tivéssemos um átomo de dois níveis, a absorção de um campo de prova apresentaria um perfil Lorentziano, tomando seu máximo valor quando o campo é ressonante com a transição atômica, ou seja, $\delta=0$ como mostra a figura 3.2.(a) em linha azul. A situação é diferente quando consideramos o caso de três níveis. De acordo com a eq.(3.20), a absorção 
nesse caso é nula na ressonância de dois fótons, representado em vermelho na figura (a). Isto se dá devido ao fato do acoplamento do campo de controle alterar a resposta do meio, tal que ao sondar com o campo de prova, pela interferência destrutiva das excitações óticas, a absorção é $\operatorname{Im} \chi^{(1)}=0$ quando a ressonância de dois fótons é $\delta=0$. Desta forma, o meio se torna transparente à luz.

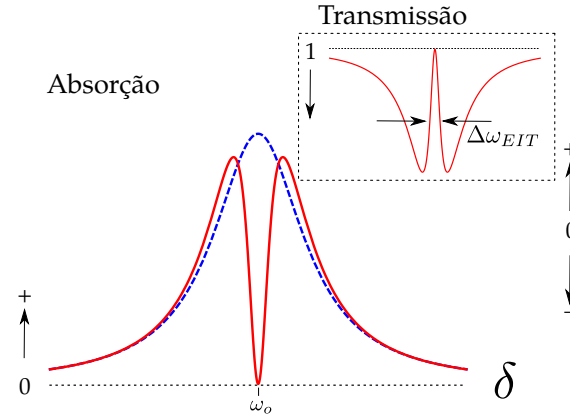

(a)

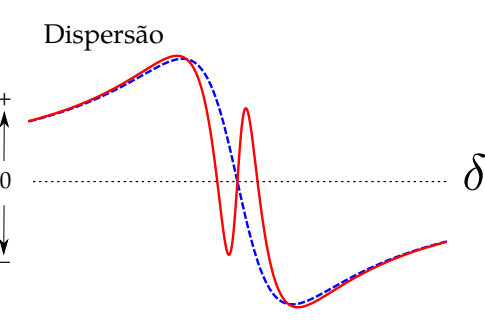

(b)

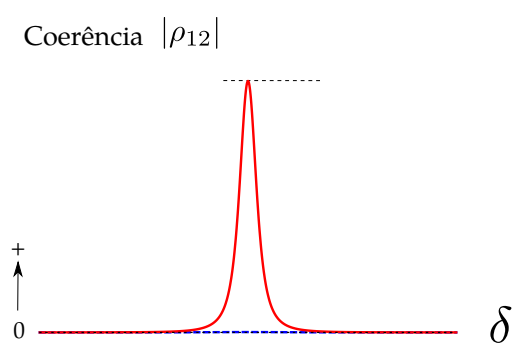

(c)

Figura 3.2: Traçamos para um sistema de dois e três níveis em azul e vermelho respectivamente, (a) a absorção do campo de prova determinada a partir de $\operatorname{Im} \chi^{(1)},(b)$ a dispersão do campo de prova determinada por Re $\chi^{(1)}$ e (c) o valor absoluto da coerência entre os níveis fundamentais. O quadro interno da figura (a) mostra a transmissão para uma densidade ótica $\sqrt{\alpha L}=1$.

Em 1976 Alzetta et al [71] reportaram CPT pela primeira vez e só em 1991 Boller et al [18] observaram pela primeira vez EIT em átomos de Estrôncio. A transparência do feixe de prova é caracterizada pela janela espectral $\Delta \omega_{E I T}$, que indica a largura espectral para a qual o meio é transparente à luz, como mostra a figura 3.2.(a). A transmissão do feixe de prova, após se propagar ao longo de uma distância $L$ do meio, é dada por $T=\exp \left(-k L \operatorname{Im} \chi^{(1)}\right)$. Dela se deduz a janela de transparência $\Delta \omega_{E I T}=2\left|\Omega_{c}\right|^{2} /(\Gamma \sqrt{\alpha L})[65]$, que revela o alargamento por potência devido à intensidade do campo de controle $\left|\Omega_{c}\right|^{2}$. Elementos como a taxa de decaimento do estado excitado $\Gamma$ e a densidade ótica ${ }^{2}$ do meio $\alpha L$, também definem a janela espectral de transparência.

No que diz respeito à dispersão, também há diferenças entre o sistema de dois e três níveis. A figura 3.2.(b) compara o caso de três níveis (em vermelho) ao caso de dois níveis

\footnotetext{
${ }^{2}$ Esta densidade ótica é definida a partir da seção transversal atômica $\alpha$ e do comprimento L, ao longo do qual a luz se propaga no meio.
} 
(em azul). Podemos notar que, para o caso de três níveis, há uma mudança abrupta em torno da ressonância $\delta=0$. Dado que o índice de refração é $n=\sqrt{1+\operatorname{Re} \chi^{(1)}}$, a velocidade de grupo é definida como

$$
v_{g}=\left.\frac{d \omega}{d k}\right|_{\delta=0}=\frac{c}{n+\omega_{p} \frac{d n}{d \omega_{p}}}
$$

De modo que, para um pulso de luz que se propaga em um meio de atômico de três níveis em condição de EIT, a variação do índice de refração é $d n /\left.d \omega_{p}\right|_{\delta=0} \gg 0$, como mostra a figura 3.2.(b). Isto se traduz em uma redução na velocidade de grupo do pulso de luz, tal que $v_{g} \ll c$ [72]. L. Hau et al. em [73] reportaram pela primeira vez uma redução da velocidade da luz para $17 \mathrm{~m} / \mathrm{s}$ em um condensado de Bose-Einstein de átomos de ${ }^{87} \mathrm{Rb}$ a 450nK. A possibilidade de diminuir a velocidade da luz em uma interface como essa, faz do fenômeno de EIT uma das principais técnicas para armazenamento de informação em meios atômicos.

Por último, é importante notar que a coerência atômica

$$
\left|\tilde{\rho}_{12}\right|=\left|\frac{\Omega_{c} \Omega_{p}}{\left(i \Delta_{p}+\Gamma\right)\left(i \delta+\gamma_{d}\right)+\Omega_{c}^{2}}\right|,
$$

atinge seu máximo valor na ressonância de transparência, como mostra a figura 3.2.(c). Isto significa que há uma superposição dos estados fundamentais, na qual potencialmente podemos armazenar informação, já que a baixa perda de coerência permite ter controle nos tempos de armazenamento, como foi discutido na seção 2.2. Isto mostra a principal razão pela qual o fenômeno de EIT é um excelente candidato a interface átomo-luz para armazenamento de luz. Protocolos como os descritos em [3, 28, 30] descrevem técnicas para armazenar pulsos de luz durante um intervalo de tempo controlado pela intensidade do campo de controle, excitando a variável de spin, ou seja, as coerências dos estados fundamentais, para em seguida, recuperar a luz desexcitando o meio atômico. 


\subsection{Transformação de ruído de fase para ruído de amplitude (RF-RA)}

Para realizar estas experiências de EIT, geralmente é empregado o laser de diodo. Eles são econômicos, práticos e logisticamente versáteis (diferente ao laser de corante e ao Titânio safira), que emitem luz com uma largura espectral fina $(\sim 100 \mathrm{kHz}-1 \mathrm{MHz})$ e no comprimento de onda da maioria dos átomos alcalinos empregados neste tipo de experiencias. O laser de diodo é conhecido por possuir uma amplitude muito estável, porém, apesar de possuir uma largura de linha pequena $(\sim 100 \mathrm{kHz}-1 \mathrm{MHz})$, ele apresenta um excesso de ruído na sua fase $^{3}[74,75]$. Portanto, cabe perguntar como esse excesso nas flutuações de fase alteram a resposta atômica.

Para entender como essa resposta atômica muda, podemos começar considerando um campo sinusoidal com uma fase estável interagido com átomos de dois níveis(ver figura 3.3.(a)). Portanto, como vimos na seção 2.1, teremos uma parte da polarização que oscila em fase com o campo e uma outra que oscila fora de fase atenuando o campo (ou seja, absorvendo-o). No entanto se, por exemplo, alteramos a fase do campo em $\pi$ em um instante de tempo $t_{1}$, como mostra figura 3.3.(b), é de se esperar que a resposta do meio mude.

Este tipo de experiência foi estudada por Camparo et al. em [76], em sistema de dois níveis acoplados por micro-ondas. O resultado é apresentado na figura 3.3.(e) e mostra como a transmissão de um feixe de prova apresenta um aumento na absorção quando a fase do campo muda em $\pi$, seguida de oscilações que se atenuam no tempo. Ao mudar novamente a fase, a intensidade apresenta novamente um comportamento transitório. Neste caso, o que acontece é que a parte da polarização que estava em fase com o campo de repente se encontra fora de fase. Portanto, a polarização se opõem à oscilação do campo atenuando-o e induzindo assim aumento na absorção. A polarização do átomo terá então uma resposta transitória, tendo vários ciclos de $\mathrm{Rabi}^{4}$ até entrar em fase com o campo novamente, atin-

\footnotetext{
${ }^{3}$ No caso do laser de titânio safira, ele não apresenta excesso de ruído na sua fase. O estado da luz emitida por esse tipo de laser pode ser descrito de forma muito próxima à do estado coerente, descrito na seção 1.9.

${ }^{4}$ Uma oscilação de Rabi é a evolução cíclica entre o nível fundamental e o nível excitado de um átomo de dois níveis. A frequência dessa oscilação para um sistema de dois níveis é dada por $\Omega=\sqrt{\Omega_{R}^{2}+\Delta^{2}}$ onde
} 
(a)
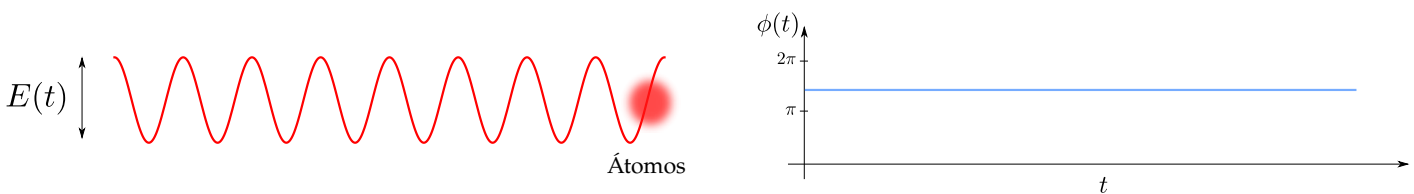

(b)

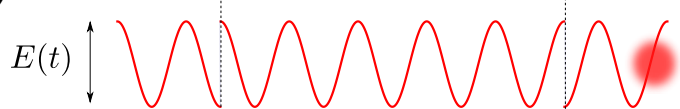

(c)

$t_{1}$
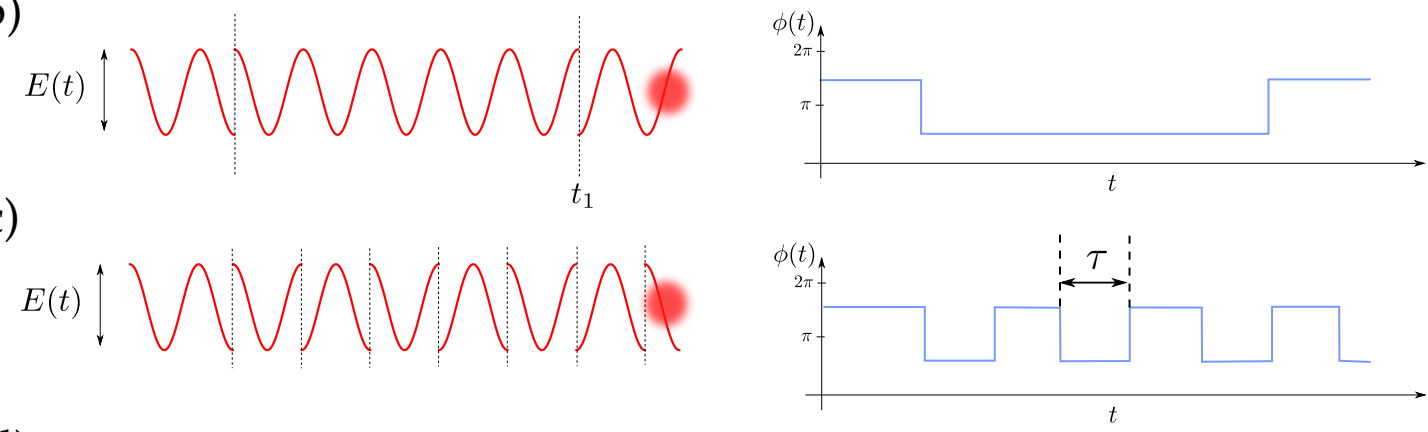

(d)
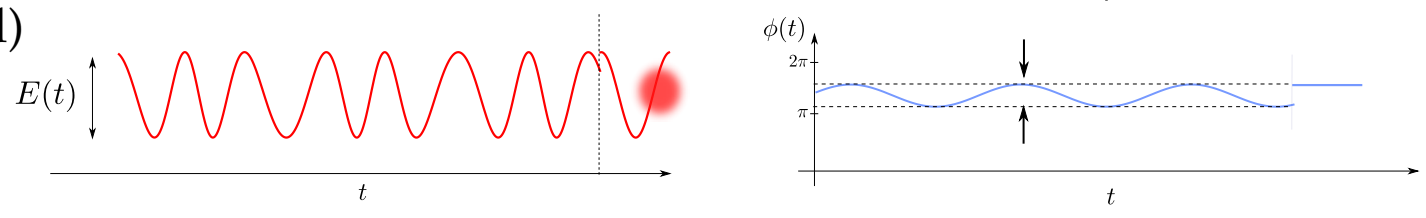

(e)
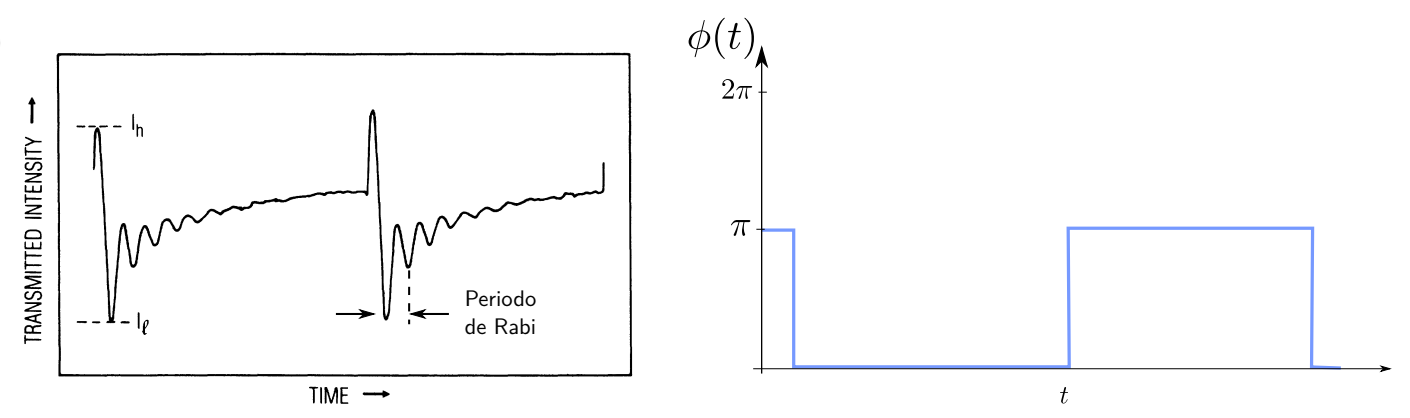

Figura 3.3: (a) Onda monocromática cuja fase é estável ao interagir com átomos. (b) Onda monocromática cuja fase é alterada em $\pi$ para dois instantes de tempo diferentes. (c) Onda monocromática cuja fase é alterada em $\pi$ sequencialmente por pulsos quadrados com período $\tau$. (d) Onda cuja fase é alterada em $\Delta \phi$ mediante uma função senoide. (e) Resposta transitória da polarização atômica com relação à variação de fase $\pi$ do campo, reportada em [76].

gindo uma condição estacionária até ele ser novamente perturbado. Esta foi uma das primeiras experiências que mostra como uma mudança na fase do campo pode ser observada na intensidade de um campo de prova.

Em seguida consideramos o caso em que mudamos a fase do campo em $\pi$ frequentemente com um sinal quadrado, como mostra a figura 3.3.(c). Neste caso, a resposta tran$\Omega_{R}$ é a frequência de Rabi associada à intensidade do campo e $\Delta$ é a dessintonia do campo com respeito à ressonância atômica. 
sitória dos átomos vai depender da frequência $1 / \tau$ do sinal quadrado com que modulamos a fase. Se o período $\tau$ for maior do que período de Rabi, teremos uma sequência de respostas transitórias como a da figura 3.3.(e). No entanto, se o tempo $\tau$ for da ordem do período de Rabi, a absorção vai aumentar significativamente. Isto ocorre devido ao fato de que ao mudar a fase do campo, o átomo está fora de fase e, portanto, aumenta absorção. Antes de terminar um ciclo de Rabi, a fase muda novamente, o átomo fica fora de fase e, portanto, o átomo absorve por um tempo mais prolongado, sujeito à saturação dos átomos. Desta forma, ao modular a fase com uma frequência $1 / \tau$, tal que $\tau$ seja da ordem do período de Rabi, obtemos então a maior amplitude de absorção na intensidade do campo de prova. Isso é o que se define como uma ressonância de Rabi [76,77]. Finalmente, para frequências muito maiores do que a frequência de uma oscilação de Rabi, a amplitude da absorção será menor do que em todos os casos anteriores, porque efetivamente, é como se o átomo não estivesse fora de fase e, portanto, não há atenuação do campo de prova.

Podemos analisar uma situação um pouco mais arbitrária em que a fase do campo é modulada por uma senoide como mostra a figura 3.3.(c), porém com uma amplitude de modulação menor a $\pi$. Nesse caso, o meio absorverá em maior ou menor medida dependendo com a frequência em que é modulada a fase. Vários trabalhos experimentais e teóricos foram feitos analisando esse tipo de efeito, em particular as refs. [76, 77].

Temos uma quarta situação em que a fase pode flutuar de forma aleatória, induzindo flutuações aleatórias na transmissão de um campo de prova. É aqui onde começamos analisar o tipo de situação que estudamos nesta tese, pois são as flutuações na fase do laser de diodo as que serão transformadas em flutuações de amplitude pelo meio atômico. Em 1991, Yabuzaki et al. [35] reportaram o primeiro experimento desse tipo. Em particular, eles mediram as flutuações do sinal transmitido por uma amostra de Césio empregando um laser de diodo. Tipicamente, para medir o espectro de absorção de um meio atômico, bastava com fazer um modulação ativa na frequência do laser ao longo de um certo intervalo de frequências. A transmissão (que está relacionada com a absorção) é registrada mediante um fotodetector, de modo que a atenuação do valor médio da intensidade $\langle I\rangle$ indica as linhas de absorção do meio. Como os fotodetetores são sensíveis unicamente à intensidade da luz, o 
espectro registrado por $\langle I\rangle$ não contém nenhuma informação de fase do sistema. Então eles adicionaram mais um elemento nessa montagem tradicional para realizar a espectroscopia: o sinal obtido no fotodetetor é demodulado por um analisador de espectros e, assim, mede o espectro de ruído da luz que chega no fotodetetor, como mostra a figura 3.4.(a). A figura 3.4.(b) mostra o espectro de ruído feita por Yabuzaki etal. para duas transições hiperfinas da linha D2 de átomos ${ }^{133}$ Cs: a transição de $F=4 \rightarrow F=5$ e a transição de cross-over $F=3 \rightarrow F=5$.
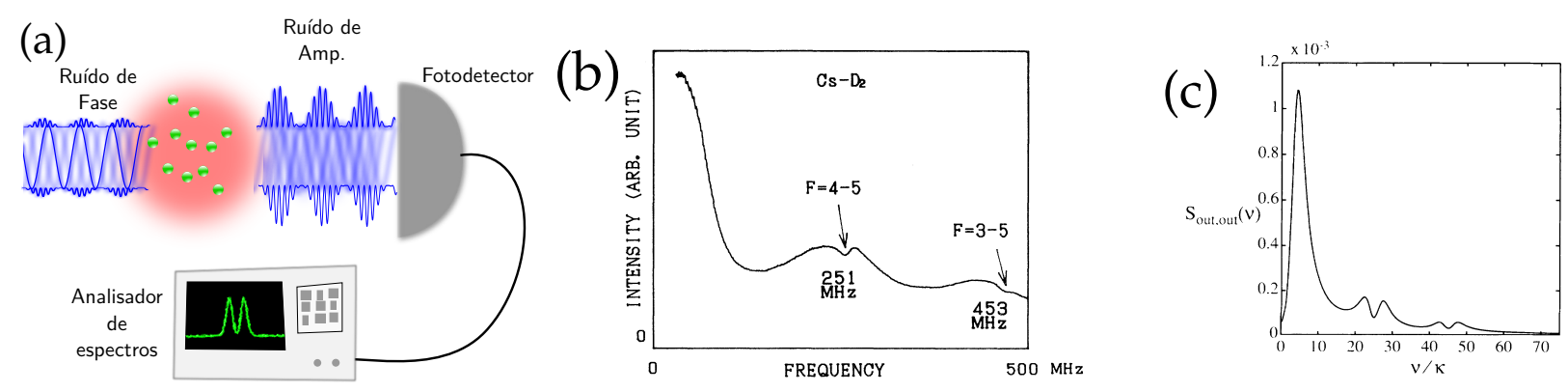

Figura 3.4: (a) Espectroscopia de intensidade obtida por Yabuzaki et.al. (b) Espectroscopia de intensidade calculada por R. Walser e P.Zoller. (c) Representação do mecanismo RF-RA, em que a luz, após interagir com os átomos, aumenta as flutuações na amplitude do campo. A intensidade da luz é medida por um fotodetetor, traduzindo-se em um sinal de fotocorrente que é processa o por um analisador de espectros.

Ao observar o sinal registrado no analisador de espectros, eles notaram que ruído de intensidade da luz $\left\langle(I-\langle I\rangle)^{2}\right\rangle$, em vez de apresentar uma atenuação, como acontece com o valor médio do sinal de intensidade $\langle I\rangle$, apresentava um aumento ao passar pelas ressonâncias atômicas. Eles atribuíram a esse excesso de ruído na transmissão a resposta atômica em interação com um laser que apresenta excesso de ruído de fase (flutuações na fase). Em outras palavras, o excesso de ruído de fase no laser é transformado pelos átomos em ruído de amplitude quando se analisam as frequências próximas das ressonâncias atômicas. Esta é então a descrição qualitativa do mecanismo de transformação de ruído de fase para ruído de amplitude (RF-RA), como ilustra a figura 3.4.(a).

Em 1994, R. Walser e P. Zoller [75] analisaram teoricamente essa transformação de ruído e como ela podia ser empregada como uma ferramenta para espectroscopia com alta precisão. 
Eles analisaram a interação de um campo de luz com fase estocástica interagindo com um átomo de dois níveis. Para isso, compararam os casos em que a fase evolui de forma difusiva e com distribuição Gaussiana real e complexa. A figura 3.4.(c) mostra os resultados teóricos para o modelo de difusão de fase, considerando os níveis hiperfinos de ${ }^{133} \mathrm{Cs}$, reproduzindo os resultados obtidos por Yabuzaki na figura 3.4.(b). Essa abordagem adota o cálculo de Itô discutido na seção 1.3.

Na mesma época, o fenômeno de EIT despertou um grande interesse da comunidade de ótica quântica, física atômica e informação quântica. Em particular, foram realizados trabalhos buscando estabelecer quais propriedades da luz em condição de EIT apresentavam um caráter intrinsecamente quântico, pois tinha-se mostrado que a interferência de excitações presente no fenômeno de EIT não era exclusivamente um efeito quântico, mas tinha um análogo clássico [32, 33]. Desenvolveram-se então alguns trabalhos teóricos para estudar verdadeiramente os efeitos quânticos para sistemas átomos-luz em condição de EIT. Duas abordagens de pesquisa foram traçadas para este tipo de sistema. Uma delas em que se analisam as propriedades quânticas em condição de EIT para campos de baixa intensidade, onde a interação de um fóton com o meio atômico revela correlações quânticas, não observadas até então. Nesse contexto, desenvolveram-se técnicas para protocolos de armazenamento de luz em meios atômicos [59] e de distribuição de emaranhamento mediante repetidores quânticos [30,31]. A segunda abordagem é quando consideramos a luz como um campo intenso, por exemplo um estado coerente. Em [17] se mostrou pela primeira vez a possibilidade de se obter estados comprimidos na quadratura de fase para um campo de prova em condição de EIT. Este tipo de efeito é de natureza claramente quântica como foi mostrado na seção 1.9. Posteriormente foram propostos alguns protocolos no regime de feixe intenso para o armazenamento de estados quânticos da luz em meios atômicos a temperatura ambiente $[20,63]$. Também foram feitos os primeiros trabalhos teóricos em que se mostra o excesso de ruído na amplitude para um sistema duplo $\Lambda$ usando EIT ou CPT $[78,79]$. No entanto, esses trabalhos teóricos levaram em conta unicamente o campo de prova de EIT ou a correlação de campos clássicos em condição CPT. Somente em 2003 foram feitas as primeiras experiências para medir a correlação entre os campos em EIT usando 
átomos à temperatura ambiente, observando algum nível de correlação quântica entre as intensidades dos campos [34].

Dado que a maioria das experiências no regime de feixes intensos ${ }^{5}$ eram feitas com laser de diodo, se percebeu que era necessário estender o mecanismo de RF-RA, desenvolvido nos trabalhos feitos por Yabuzaki e Zoller de sistemas de dois níveis, para sistemas de três níveis com campos em condição de EIT e Hanle-EIT ${ }^{6}$. No domínio da frequência, Martinelli et al. em [38] observaram o mecanismo RF-RA ao medir o ruído de intensidade para diferentes campos com polarizações ortogonais interagindo com átomos de rubídio à temperatura ambiente em condição de Hanle-EIT. Em 2007, L. Cruz et al. em [16] fizeram as primeiras medidas no domínio da frequência da correlação de intensidade entre os campos em condição de EIT, levando em conta o mecanismo RF-RA, com átomos à temperatura ambiente. Em particular, se observou que $C(\omega)$ definida na eq.(1.36) toma valores positivos $(C(\omega)>0)$ para campos fracos descrevendo assim campos com intensidades correlacionadas. Entretanto, conforme se aumenta a intensidade dos campos, então $C(\omega)<0$ e, portanto, os campos apresentam uma anti-correlação nas suas intensidades.

Por outro lado, inspirado nos trabalhos de Hanbury Brown \& Twiss, M.O. Scully liderou uma série de trabalhos $[15,36,37]$ adotando uma abordagem temporal. Nesses trabalhos se mediu a função de correlação normalizada $g^{(2)}(\tau)$, descrita na seção 1.2.2, para dois campos após interagir com átomos de rubídio à temperatura ambiente em condição de EIT $^{7}$. As experiências foram feitas com laser de diodo, em que novamente era necessário levar em conta o mecanismo RF-RA. Em particular, foram usados campos circularmente polarizados em configuração de Hanle-EIT, como mostra a figura 3.5.(a), em que temos um sistema $\Lambda$ em condição de transparência quando não há presença de campos magnéticos. Eles observaram que para tempos $\tau \sim 0$, o meio atômico induzia uma correlação $g^{(2)}(0)>0$ (ver a figura 3.5.b). Desta forma, as flutuações de intensidade $\delta I$ em cada um dos campos mudam

\footnotetext{
${ }^{5}$ Também conhecido como abordagem em variáveis contínuas

${ }^{6}$ Ao aplicar campos magnéticos em átomos com degenerescência Zeeman, existem condições para obter transparência induzida da mesma forma que em EIT.

${ }^{7} \mathrm{Na}$ eq.(1.22) se definiu a correlação entre dois campos como $\tilde{g}_{12}^{(2)}(\tau)$. No entanto, daqui para frente usaremos a notação adotada pela comunidade $[15,36,37,43]$ em que $g^{(2)}(\tau) \equiv \tilde{g}_{12}^{(2)}(\tau)$.
} 
simultaneamente e na mesma proporção como mostra a figura 3.5.(f). No entanto, ao aplicar um campo magnético quebra-se a condição Raman, isto é $\delta \neq 0$ e, portanto, os campos estão fora da janela de transparência. Nesta situação, os átomos induzem uma anticorrelação entre os campos, ou seja, $g^{(2)}(0)<0$ como mostra a figura 3.5.(c). As flutuações ocorrem na mesma proporção mas em sentido oposto, como ilustra a figura 3.5.(g). Isto é comparado aos dois casos que deixam claro o papel dos átomos: o primeiro na figura 3.5.(d), em que se bloqueia um dos campos, de modo que somente interage com um deles, e o segundo, na figura 3.5.(e), em que os feixes se propagam com uma separação de $0.3 \mathrm{~cm}$ ao longo da célula de vapor de Césio. Em nenhum desse dois casos se observa uma correlação definida como a que existe no caso (f) e (g).

(a)

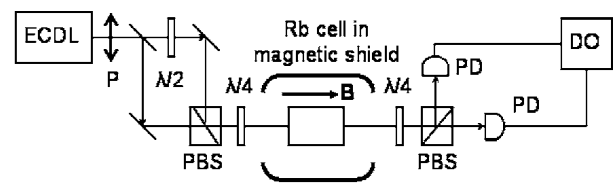

(b)

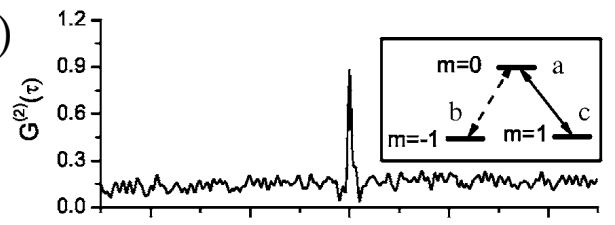

(c)

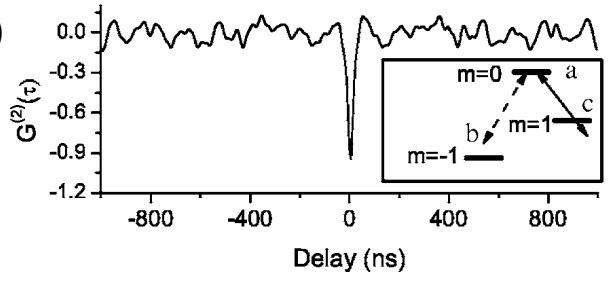

(d)

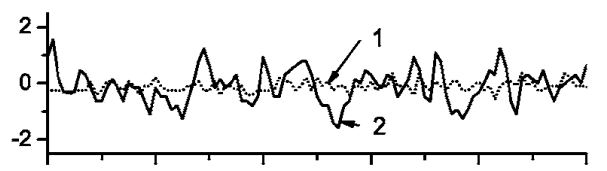

(e)

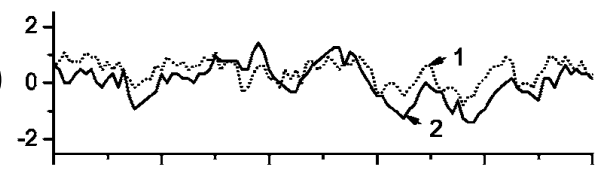

(f)
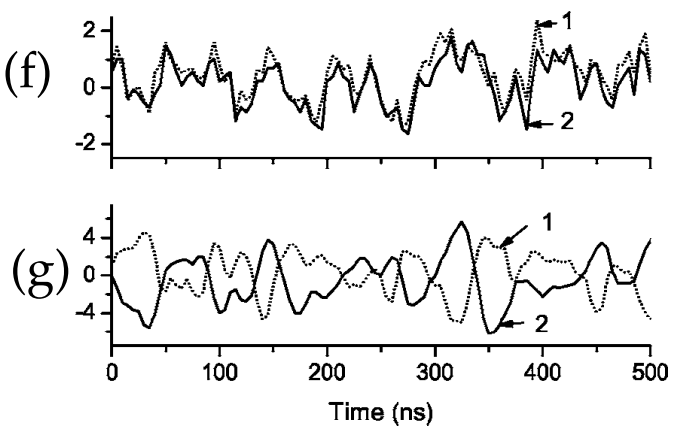

Figura 3.5: (a) Montagem experimental empregada em [37], onde os átomos de Rubídio à temperatura ambiente são colocados dentro de um solenoide e protegidos da presença de campos magnéticos externos por uma blindagem magnética. Medida da função de correlação $g^{(2)}(\tau)$ no caso em que (b) $B=0 e(c) B \neq 0$. Flutuações de intensidade para cada um dos campos denotados por 1 e 2 , quando (d) um deles é bloqueado, (e) quando os feixes tem uma separação lateral de $0.3 \mathrm{~ms}$ e, portanto, eles não se superpõem, (f) quando os campos satisfazem a condição de EIT induzindo uma correlação e (g) quando é aplicado um campo magnético que quebra a degenerescência dos níveis atômicos, induzindo uma anticorrelação.

Embora fosse claro que, ao se aplicar campos magnéticos para deslocar os níveis atômicos, 
permitiria-se induzir correlação ou anticorrelação entre os campos em condição de HanleEIT, não se aprofundou o entendimento sobre quais as propriedades que mudam nos átomos para eles induzirem esse tipo de correlação ou anti-correlação. Trabalhos como os publicados em $[15,40]$ estudam a correlação entre os dois campos do sistema $\Lambda$-EIT, mudando a dessintonia de dois fótons, realizando assim uma espectroscopia da função $g^{(2)}(0)$. Os espectros são apresentados na figura 3.6. Porém, o espectro da figura 3.6.(a), feito por Varzhapetyan et al. em [49], mostra que a correlação toma unicamente valores positivos para todas as dessintonias dos campos óticos ${ }^{8}$. Entretanto, a espectroscopia da figura 3.6.(b), feita por Xiao et al. em [40], mostra que existe uma possível transição de correlação para anticorrelação à medida que aumenta a dessintonia de dois fótons $\delta \in[-10 k \mathrm{~Hz}, 0 \mathrm{kHz}]$. Desta forma podemos questionar: quais são as condições do sistema átomo-luz para que exista correlação ou anticorrelação entre os campos em condição de EIT? Era necessário então, fazer um mapeamento dos parâmetros atômicos para explicar como os átomos induzem correlação e anticorrelação entre os campos.
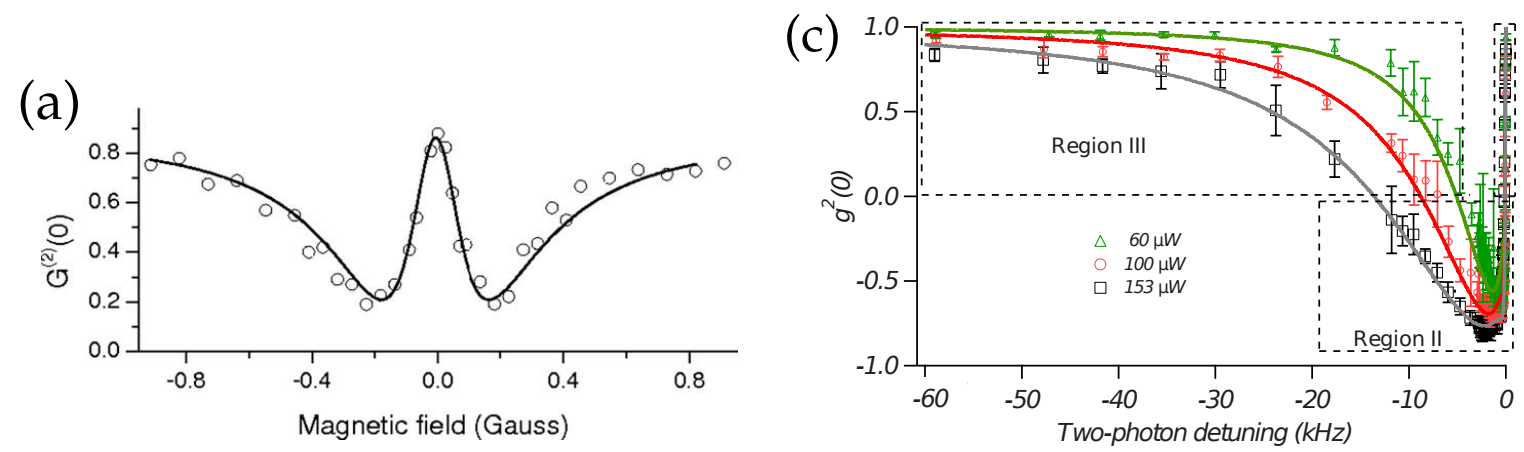

Figura 3.6: Função de correlação normalizada $g^{(2)}(0)$ em função de: (a) o campo magnético, medido em [37]. (b) Em função da dessintonia de dois fótons medido em [40].

Contudo, os modelos usados tanto no domínio das frequências em [16, 38] quanto no domínio temporal em [15, 36, 37, 40], fornecem soluções numéricas, que no entanto carecem de uma forma analítica que mostre explicitamente o papel das variáveis atômicas na correlação dos campos. Em ambos os casos, o fato de lidar com átomos à tempera-

\footnotetext{
${ }^{8}$ Aplicar campos magnéticos neste tipo de sistemas se traduz em aplicar uma dessintonia de dois fótons nos campos óticos. A figura 3.6.(a) apresenta a função $g^{(2)}(0)$ em função do campo magnético, que equivale a mudar a dessintonia de dois fótons como é feito na figura 3.6.(b).
} 
tura ambiente obriga a inclusão do efeito Doppler na interação átomo-luz, o que dificulta a obtenção de uma solução analítica para a correlação. Uma segunda questão é a modelagem das flutuações estocásticas da fase. No caso do domino da frequência, seguiu-se a abordagem de Zoller, em que se empregou o cálculo de Itô, descrito na seção 1.3.2, para calcular a correlação. No entanto, a abordagem dada até então impossibilita achar um solução analítica em termos dos parâmetros da interação luz-átomos como a dessintonia, frequência de Rabi, taxa de decaimento e largura de banda dos campos óticos (ruído de fase). No caso temporal, apesar de terem sido feitos avanços em relacionar diretamente a correlação com elementos da matriz dos átomos usando o mecanismo RF-RA, não se mostrou uma expressão analítica que descrevesse a correlação diretamente em termos da dessintonia, etc. Isto permitiria achar uma relação entre a correlação e as propriedades como a dispersão e absorção dos átomos, que representam os principais elementos de caracterização para registrar a resposta atômica.

Em 2013 publicamos o trabalho [44] ${ }^{9}$, em que fizemos um estudo aprofundado da correlação dos campos interagindo com átomos em condição de EIT. Em particular, usamos átomos frios de duas espécies diferentes: Rubídio e Césio. Pelo fato de serem frios, nos permitiu contornar a dificuldade que o efeito Doppler introduz na modelagem da correlação do sistema físico que vamos estudar. Nesse trabalho, fizemos uma abordagem no domínio do tempo e da frequência. No domínio do tempo, observou-se o mesmo tipo de correlação e anticorrelação como na figura 3.5. No domínio da frequência, estudamos teórica e experimentalmente a espectroscopia de absorção, de ruído de intensidade e de correlação dos campos. Em ambos os casos, reportamos a transição de um regime de correlação para anticorrelação conforme a intensidade dos campos aumentava. As figuras 3.7.(a) e (b) mostram a espectroscopia de correlação para átomos de ${ }^{133} \mathrm{Cs} \mathrm{e}{ }^{85} \mathrm{Rb}$, respetivamente. Os espectros mostram o comportamento da correlação para várias intensidades dos campos em condição $\Lambda$-EIT. Mostramos então que, para intensidades baixas suficiente, (traçados em preto e azul) os campos estão sempre correlacionados para qualquer valor de dessintonia de um dos campos, reproduzindo a condição obtida por Scully na figura 3.6.(a).

\footnotetext{
${ }^{9}$ Em colaboração com o grupo de átomos Frios do Departamento de Física da UFPE
} 


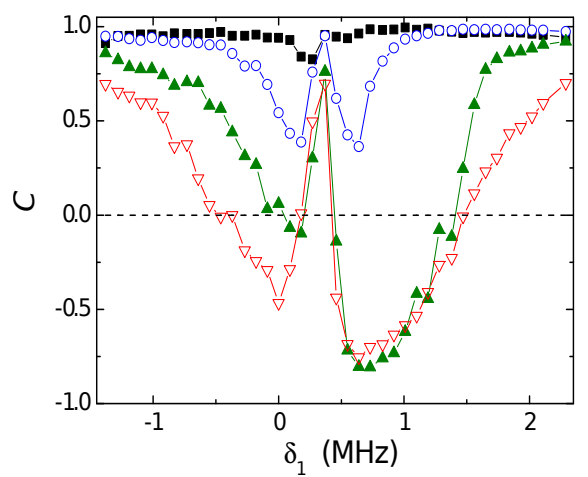

(a)

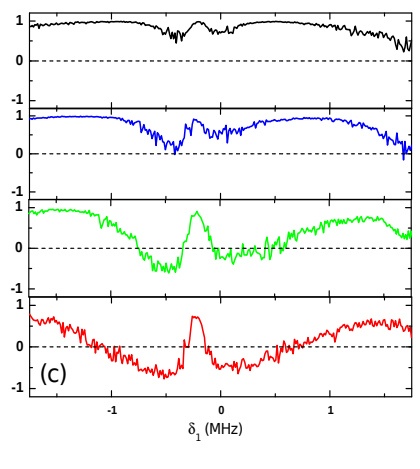

(b)

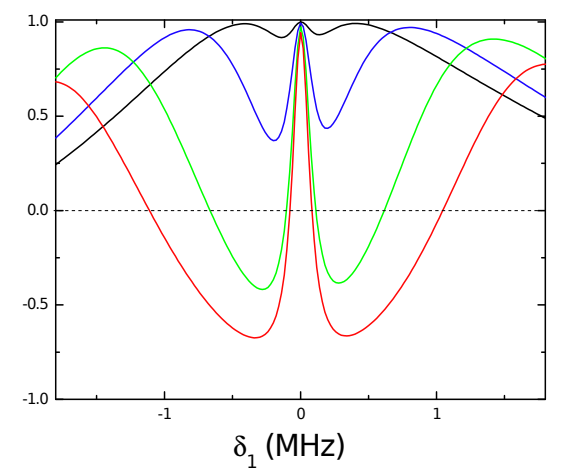

(c)

Figura 3.7: Resultado para o coeficiente de correlação $C(\omega)$ em função da dessintonia $\delta_{1}$, em [44]. (a) Experimental para átomos de Césio. (b) Experimental para átomos de Rubídio. (c) Teórico. Em linha preta traçamos o espectro que corresponde a uma intensidade $30 I_{0}$. Para as linhas azul, verde e vermelha as intensidades dos campos são $100 I_{0}, 200 I_{0}$ e $500 I_{0}$

Entretanto, ao aumentar a intensidade dos campos, passamos de um regime de correlação para um de anticorrelação, traçado em verde e vermelho. Este tipo de espectro, em que a correlação toma valores negativos, coincide com o que foi reportado por Xiao na figura 3.6.(b). A figura3.7.(c) mostra o resultado teórico da correlação usando o cálculo de Itô como foi feito por Zoller e estendido para um sistema de três níveis como foi feito em [16].

Uma das características mais importantes dos espectros, é a largura espectral próxima da ressonância $\delta_{1} \sim 0$. Acontece que tipicamente, os espectros de absorção sempre apresentam alargamento por potência, ou seja, o alargamento espectral da absorção devido à saturação do meio quando a intensidade do campo aumenta. A figura 3.8 mostra (em linha preta) o valor esperado para a largura do espectro da absorção em função da intensidade dos campos $^{10}$. Isto mostra que o aumento da largura da ressonância de EIT (tanto experimental quanto teórico) é linearmente proporcional à intensidade dos campos. No entanto, em vermelho mostramos que na ressonância de EIT, a correlação não é sensível ao alargamento por potência, porque a largura intrínseca se mantêm relativamente constante à medida que aumenta a intensidade dos campos ${ }^{11}$. Este foi um dos resultados mais importantes do traba-

\footnotetext{
${ }^{10}$ A largura da janela de transparencia de EIT $\Delta \omega_{E I T}$ é medida como a largura a média altura entre o ponto de dessintonia de EIT e os dois pontos de máxima absorção

${ }^{11}$ A largura intrínseca da correlação é medida como a largura a media altura entre o ponto de dessintonia $\delta=0$ e os dois pontos de menor correlação mais próximos da ressonância, como mostra a figura 3.7
} 
lho, pois mostra que o espectro de correlação permite medir a largura intrínseca da janela de transparência de EIT, independente do valor de intensidade dos campos.
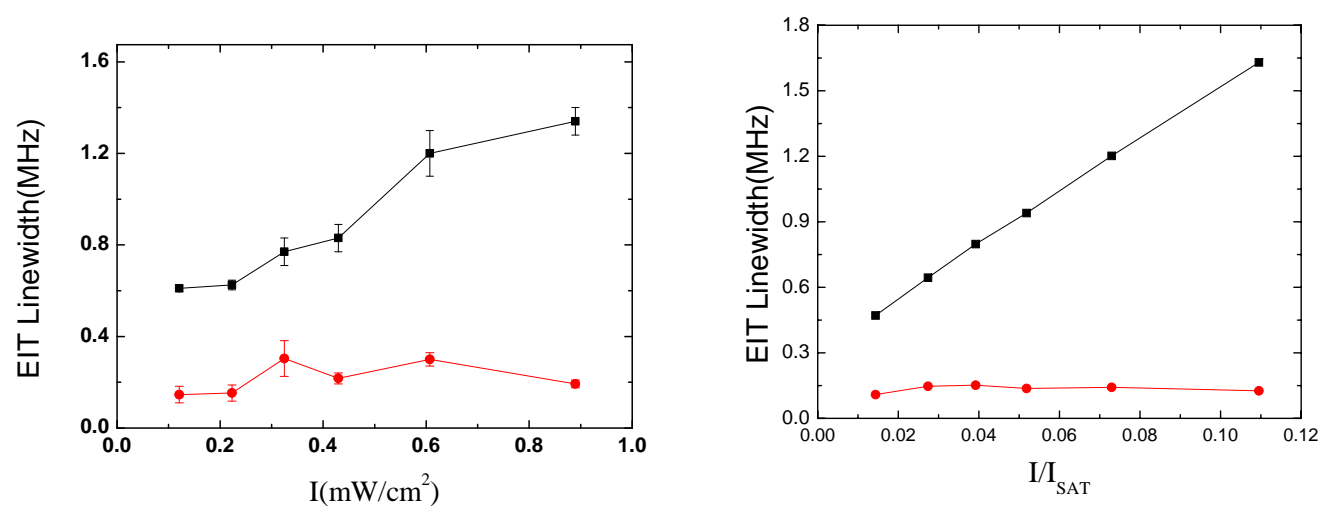

Figura 3.8: Resultado experimental (esquerda) e teórico (direita) comparando o alargamento por potência da largura de EIT medida pela transmissão e da largura intrinseca medida pelo espectro de correlação.

Isto é ideal para aplicações em metrologia, pois para medir o valor real de uma grandeza física é importante eliminar as possíveis fontes que contaminem a medida. Embora no aspecto teórico tenhamos conseguido reproduzir estas mesmas características com o modelo implementado em [16], ainda temos o desafio de achar um modelo que nos conduza a uma expressão analítica no domínio das frequências, que nos permita estabelecer um mapeamento entre a correlação e os parâmetros como a absorção e a dispersão dos campos.

Nesse sentido, em 2013 [43], propusemos um modelo heurístico para descrever a função de correlação $g^{(2)}(0)$ em termos das propriedades atômicas. O intuito desse trabalho foi relacionar a correlação $g^{(2)}(0)$ com a parte dispersiva e a absortiva dos átomos em condição de EIT. A seguir faremos uma breve revisão deste modelo como base para analisar os resultados obtidos na seção 3.6, em que obtemos uma expressão analítica para a correlação no domínio da frequência. 


\subsection{Modelo heurístico para correlação $g^{(2)}(0)$ para EIT}

Como foi discutido na seção 1.2.2, a função de correlação normalizada de segunda ordem para dois campos $\mathbf{E}_{1}$ e $\mathbf{E}_{2}$ com intensidades $I_{1}(t)$ e $I_{2}(t)$, é descrita pela desigualdade de Cauchy-Schwarz. Aqui mostraremos o modelo heurístico proposto em [43] para determinar a função de correlação $g^{(2)}(0)$ em função das variáveis atômicas, para um sistema $\Lambda$-EIT como mostra a figura 3.9. Para isto, assumiremos que os campos $\mathbf{E}_{1}$ e $\mathbf{E}_{2}$ apresentam flutuações de amplitude e de fase clássicas. Além disso, consideremos para cada um deles a aproximação linear, de modo que as intensidades podem ser expressas como $I_{i}(t)=\left\langle I_{i}\right\rangle+\delta I_{i}(t)$, como foi descrito na seção 1.2.

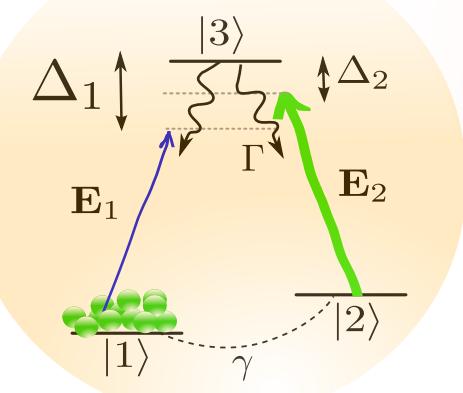

(a)

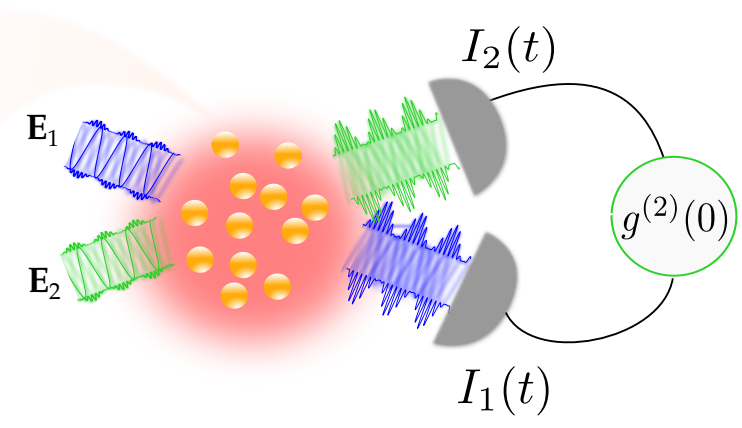

(b)

Figura 3.9: (a) Átomo de três níveis interagindo com os campos $\mathbf{E}_{1}$ e $\mathbf{E}_{2}$ em configuração $\Lambda$, com dessintonias $\delta_{1}$ e $\delta_{2}$ respectivamente, com respeito ao estado excitado. $\Gamma$ representa a taxa de emissão espontânea e $\gamma$ a taxa de decoerência entre os estados fundamentais. (b) Geometria de interação dos campos com o meio atômico e esquema de deteção de intensidade para determinar a função de correlação $g^{(2)}(0)$.

Adotando a notação em [49] temos então que a função de correlação normalizada é dada por

$$
g^{(2)}(\tau)=\frac{\left\langle\delta I_{1}(t) \delta I_{2}(t+\tau)\right\rangle}{\sqrt{\left\langle\delta I_{1}(t)^{2}\right\rangle\left\langle\delta I_{2}(t+\tau)^{2}\right\rangle}}
$$

Em particular, como foi discutido anteriormente, para este tipo de sistema a função de 
correlação apresenta as suas principais características para $\tau=0$, tal que

$$
g^{(2)}(0)=\frac{\left\langle\delta I_{1}(t) \delta I_{2}(t)\right\rangle}{\sqrt{\left\langle\delta I_{1}(t)^{2}\right\rangle\left\langle\delta I_{2}(t)^{2}\right\rangle}}
$$

Para determinar as flutuações de intensidade $\delta I_{i}$ após os campos interagirem com os átomos, partimos da eq.(2.2) para obter a propagação dos campos $\mathbf{E}_{1}$ e $\mathbf{E}_{2}$. Considerando então que os campos se propagam na direção $\hat{z}$ e, que de acordo com a eq.(2.8), a polarização atômica é $\mathbf{P}=\bar{n} \operatorname{Tr}\{\rho \mathbf{d}\}$, então para cada um dos campos temos a seguinte equação:

$$
\frac{\partial^{2} \mathbf{E}_{i}(z, t)}{\partial^{2} z}+k_{i}^{2} \mathbf{E}_{i}(z, t)=-k_{i}^{2} \bar{n} \mathbf{d}_{i 3} \rho_{i 3}(z, t)
$$

onde $k_{i}$ é o vetor de onda para cada um dos campos, $\bar{n}$ é a densidade atômica, $\mathbf{d}_{i 3}$ é o valor esperado do momento dipolar e $\rho_{i 3}(t)$ corresponde à coerência atômica associada ao acoplamento do campo $\mathbf{E}_{i}$. Consideremos também que a amplitude dos campos depende da posição, ou seja, $\tilde{\mathcal{E}}_{i}=\tilde{\mathcal{E}}_{i}(z)$. Portanto, ao tomar a solução do campo elétrico da eq.(2.1) em que $\mathbf{E}(z, t)=\tilde{\mathcal{E}}_{i}(z) e^{i(k z-\omega t+\phi)} \hat{\mathbf{e}}_{i}(\theta, \epsilon)+$ c.c, , temos então

$$
\frac{\partial^{2} \tilde{\mathcal{E}}_{i}(z)}{\partial^{2} z}+2 i k_{i} \frac{\partial \tilde{\mathcal{E}}_{i}(z)}{\partial z}=-k_{i}^{2} \bar{n} d_{i 3} \tilde{\rho}_{i 3}(z, t)
$$

onde $d_{i 3}=\hat{\mathbf{e}}_{i}(\theta, \epsilon) \cdot \mathbf{d}_{i 3}$ e $\tilde{\rho}_{i 3}(z, t)$ correspondem às coerências lentamente variáveis já definidas nas eqs.(3.10)-(3.11). Assumindo a aproximação paraxial em ambos os campos tal que $\left|\partial_{z}^{2} \tilde{\mathcal{E}}\right| \ll k\left|\partial_{z} \tilde{\mathcal{E}}_{i}\right|$, a equação de propagação da amplitude lentamente variável $\mathcal{E}_{i}$ é dada por

$$
\frac{\partial \tilde{\mathcal{E}}_{i}(z)}{\partial z}=i \frac{n k_{i} d_{i 3}}{2} \tilde{\rho}_{i 3}(z, t)
$$

Dado que a amostra atômica empregada no experimento desta tese se caracteriza por ter uma baixa densidade, podemos então adotar a aproximação de amostra fina. Com ela consideramos que $\tilde{\rho}_{i 3}$ não depende da posição, o que implica que o campo, após interagir com o meio, não sofre uma atenuação abrupta na sua amplitude tal que $\tilde{\mathcal{E}}_{i}(z) \approx \tilde{\mathcal{E}}_{i}(0)$. Portanto, 
temos que a solução da eq.(3.27) é

$$
\tilde{\mathcal{E}}_{i}(L) \approx \tilde{\mathcal{E}}_{i}(0)+i \bar{\kappa}_{i} \tilde{\rho}_{i 3}(t)
$$

onde $\bar{\kappa}_{i}=n k_{i} d_{i 3} L / 2$. Portanto, a intensidade dos campos transmitidos é dada por

$$
I_{i}(t)=\left|\tilde{\mathcal{E}}_{i}(L)\right|^{2}=\left|\tilde{\mathcal{E}}_{i}(0)\right|^{2}+i \bar{\kappa}_{i} \tilde{\rho}_{i 3}(t) \tilde{\mathcal{E}}_{i}(0)-i \bar{\kappa}_{i} \tilde{\rho}_{3 i}(t) \tilde{\mathcal{E}}_{i}(0)+\left|\bar{\kappa}_{i} \tilde{\rho}_{i 3}\right|^{2}
$$

Dado que estamos assumindo a aproximação de amostra fina, podemos desprezar o último termo da eq.(3.29) proporcional a $\left|\bar{\kappa}_{i}\right|^{2}$, de modo que

$$
I_{i}(t)=\left|\tilde{\mathcal{E}}_{i}\right|^{2}-2 \bar{\kappa}_{i} \operatorname{Im}\left\{\tilde{\rho}_{i 3}(t) \tilde{\mathcal{E}}_{i}\right\}
$$

onde temos denotado $\tilde{\mathcal{E}}_{i}=\tilde{\mathcal{E}}_{i}(0)$. Portanto, tomando $\left\langle I_{i}\right\rangle=\left|\tilde{\mathcal{E}}_{i}\right|^{2}$, a flutuação de intensidade para cada um dos campos é

$$
\delta I_{i}(t)=I_{i}(t)-\left\langle I_{i}\right\rangle=-2 \bar{\kappa} \operatorname{Im}\left\{\tilde{\rho}_{i 3}(t) \tilde{\mathcal{E}}_{i}\right\}
$$

Acabamos de descrever a propagação dos campos ao longo de uma distância $L$ dentro do meio. As intensidades na sua saída são dadas pela eq.(3.30) e as suas flutuações pela eq.(3.31). Elas podem se propagar no espaço livre até serem detetadas para medir a sua correlação e determinar como ela muda pelos parâmetros atômicos.

\section{Mecanismo RF-RA}

Se os campos não apresentarem uma difusão na sua fase, podemos tomar a amplitude $\tilde{\mathcal{E}}$ como variável real, e de acordo com a eq.(3.31), as flutuações de intensidade dependem unicamente da absorção atômica, tal que

$$
\delta I_{i}(t)=I_{i}(t)-\left\langle I_{i}\right\rangle=-2 \bar{\kappa} \tilde{\mathcal{E}}_{i} \operatorname{Im} \tilde{\rho}_{i 3}(t)
$$


Agora, se os campos apresentarem difusão de fase $\varphi$, então isso se traduz em $\tilde{\mathcal{E}} \rightarrow \tilde{\mathcal{E}} e^{i \varphi}$, e portanto temos

$$
\delta I_{i}(t)=c_{o}\left[\cos \varphi \operatorname{Im} \tilde{\rho}_{i 3}(t)+\sin \varphi \operatorname{Re} \tilde{\rho}_{i 3}(t)\right]
$$

em que $c_{o}=-2 \kappa_{i} \tilde{\mathcal{E}}$, onde $\operatorname{Im} \rho_{i 3}(t)$ e $\operatorname{Re} \rho_{i 3}(t)$ correspondem à parte absortiva e dispersiva dos dois campos $i=1,2$. Portanto, mostramos que pela interação dos campos com o meio atômico, a absorção e a dispersão devida aos átomos transformam a difusão de fase (ruído de fase) em ruído de amplitude proporcional a $\delta I$. Isto é muito importante, porque os fotodetetores por serem sensíveis unicamente à amplitude do campo e insensíveis à fase, a transformação RF-RA que ocorre na interação da luz com os átomos faz com que a difusão de fase se torne "visível" aos detetores. É fundamental caracterizar este tipo de mecanismo no contexto de ótica quântica e informação quântica, já que por meio dele é possível contaminar informação codificada nas quadraturas de fase e amplitude do campo.

Para determinar a correlação entre os campos dada na eq.(3.24), é necessário calcular a média do produto das flutuações de intensidade com relação à difusão de fase, tal que

$$
\left\langle\delta I_{i}(t) \delta I_{j}(t)\right\rangle_{\varphi}=\frac{c_{o}^{2}}{2}\left[\operatorname{Im} \tilde{\rho}_{i 3}^{e s}(t) \operatorname{Im} \tilde{\rho}_{j 3}^{e s}(t)+\operatorname{Re} \tilde{\rho}_{i 3}^{e s}(t) \operatorname{Re} \tilde{\rho}_{j 3}^{e s}(t)\right] \quad i, j=1,2
$$

onde levamos em conta que $\left\langle\cos ^{2} \varphi\right\rangle=\left\langle\sin ^{2} \varphi\right\rangle=1 / 2 \mathrm{e}\langle\cos \varphi \sin \varphi\rangle=0$. Para calcular o valor médio também foi considerada a solução estacionária das coerências atômicas $\rho_{i 3}^{e s}(t)$, já que temos interesse no regime em que as variáveis atômicas se mantém constantes em intervalos de tempo curtos $\tau \rightarrow 0$, nos quais se mede a correlação de flutuações. Por simplicidade, podemos redefinir as soluções estacionárias como $p_{i} \equiv \rho_{i 3}^{e s}(t)$. Assim, ao substituir os termos de ruído de intensidade dos campos $\left\langle\delta I_{i}(t)^{2}\right\rangle$ e o produto $\left\langle\delta I_{1}(t) \delta I_{2}(t)\right\rangle$ na função de correlação normalizada (3.24), temos

$$
g^{(2)}(0)=\frac{\operatorname{Im} p_{1} \operatorname{Im} p_{2}+\operatorname{Re} p_{1} \operatorname{Re} p_{2}}{\sqrt{\left(\operatorname{Im}^{2} p_{1}+\operatorname{Re}^{2} p_{1}\right)\left(\operatorname{Im}^{2} p_{2}+\operatorname{Re}^{2} p_{2}\right)}}
$$


em que adotamos a notação $\operatorname{Im}^{2} p_{i} \equiv\left(\operatorname{Im} p_{i}\right)^{2} e$ e $\operatorname{Re}^{2} p_{i} \equiv\left(\operatorname{Re} p_{i}\right)^{2}$. A expressão (3.35) mostra então como a função de correlação depende diretamente do produto das dispersões $\operatorname{Re} p_{1} \times \operatorname{Re} p_{2}$ e das absorções $\operatorname{Im} p_{1} \times \operatorname{Im} p_{2}$ dos campos devido à interação com os átomos. Para obter a solução destas variáveis atômicas, resolvemos as equações de Bloch (3.7)-(3.12). Para isso, consideramos o sistema isolado, de tal forma que os estados atômicos satisfazem a condição de normalização

$$
\tilde{\rho}_{11}+\tilde{\rho}_{22}+\tilde{\rho}_{33}=1
$$

Ao expressar a população do estado excitado como $\tilde{\rho_{33}}=1-\tilde{\rho}_{11}-\tilde{\rho}_{22}$, as eqs.(3.7)-(3.8) podem ser reescritas assim

$$
\begin{aligned}
& \dot{\tilde{\rho}}_{11}=i \Omega_{1}\left(\tilde{\rho}_{13}-\tilde{\rho}_{31}\right)+\Gamma\left(1-\tilde{\rho}_{11}-\tilde{\rho}_{22}\right), \\
& \dot{\tilde{\rho}}_{22}=i \Omega_{2}\left(\tilde{\rho}_{23}-\tilde{\rho}_{32}\right)+\Gamma\left(1-\tilde{\rho}_{11}-\tilde{\rho}_{22}\right) .
\end{aligned}
$$

Portanto, ao definir o vetor das variáveis atômicas $X=\left\{\tilde{\rho}_{11}, \tilde{\rho}_{22}, \tilde{\rho}_{13}, \tilde{\rho}_{31}, \tilde{\rho}_{32}, \tilde{\rho}_{23}, \tilde{\rho}_{12}, \tilde{\rho}_{21}\right\}$, as equações de Bloch podem ser escritas de forma vectorial como

$$
\frac{d X}{d t}=\mathbf{A}_{t} \cdot X+y_{0}
$$

onde definimos o vetor $y_{0}=\left\{\Gamma, \Gamma,-i \Omega_{1}, i \Omega_{1}^{*},-i \Omega_{2}, i \Omega_{2}^{*}, 0,0\right\}$ e a matriz $\mathbf{A}_{t}$ como

$$
\mathbf{A}_{t}=\left(\begin{array}{cccccccc}
-\Gamma & -\Gamma & i \Omega_{1}^{*} & -i \Omega_{1} & 0 & 0 & 0 & 0 \\
-\Gamma & -\Gamma & 0 & 01 & i \Omega_{2}^{*} & -i \Omega_{2} & 0 & 0 \\
2 i \Omega_{1} & i \Omega_{1} & -\left(i \Delta_{1}+\Gamma\right) & 0 & 0 & 0 & i \Omega_{2} & 0 \\
-2 i \Omega_{1}^{*} & -i \Omega_{1}^{*} & 0 & \left(i \Delta_{1}-\Gamma\right) & 0 & 0 & 0 & -i \Omega_{2} \\
-2 i \Omega_{2}^{*} & -i \Omega_{2}^{*} & 0 & 0 & \left(i \Delta_{2}-\Gamma\right) & 0 & 0 & i \Omega_{1}^{*} \\
2 i \Omega_{2} & i \Omega_{2} & 0 & 0 & 0 & -\left(i \Delta_{2}+\Gamma\right) & -i \Omega_{1} & 0 \\
0 & 0 & i \Omega_{2}^{*} & 0 & -i \Omega_{1} & 0 & -\left(i \delta+\gamma_{d}\right) & 0 \\
0 & 0 & 0 & -i \Omega_{2} & i \Omega_{1}^{*} & 0 & 0 & \left(i \delta-\gamma_{d}\right) \\
& & & & & & &
\end{array}\right)
$$

Ao considerar o regime estacionário do sistema, teremos que $\frac{d X}{d t}=0$, tal que mediante a 
inversa da matriz $\mathbf{A}_{t}$, podemos determinar

$$
X_{\mathrm{es}}=-\left(\mathbf{A}_{t}^{-1}\right) y_{0}
$$

que corresponde à solução estacionária das populações e coerências atômicas. A partir desta solução podemos obter a dispersão e absorção $\operatorname{Re} \tilde{\rho}_{i 3}^{e s}(t)=\operatorname{Re} p_{i}$ e $\operatorname{Im} \tilde{\rho}_{13}^{e s}(t)=\operatorname{Im} p_{i}$, respectivamente, necessárias para determinar a função de correlação (3.35).

A figura 3.10.(a) mostra a função de correlação em função da dessintonia $\Delta_{1}$ a partir do resultado da eq.(3.35) usando a solução estacionária (3.41). Foram traçados quatro espectros da função de correlação para frequências de Rabi $\Omega_{1}=0.1 \Gamma, 0.2 \Gamma, 0.3 \Gamma, 0.4 \Gamma$, com $\Omega_{2}=\Omega_{1}$. O resultado teórico traça o mesmo comportamento obtido experimentalmente em [43]. Notamos que a função de correlação mostra a transição de um regime de correlação $g^{(2)}(0)>0$, traçada em linha verde para $\Omega_{1}=0.1 \Gamma$, até atingir um regime em que é possível ter anticorrelação $g^{(2)}(0)<0$, traçada em azul. Os dois regimes se ajustam aos casos apresentados na figura 3.6 para os resultados de Scully e Xiao. O espectro de correlação é caracterizado pela largura intrínseca $\Delta \omega_{\text {Int }}$, que como foi comentado na seção anterior, é livre de alargamento por potência. Nesse trabalho mostramos que ela despende diretamente da taxa de decoerência, ou seja, $\Delta \omega_{\text {Int }} \approx \gamma_{d}$.

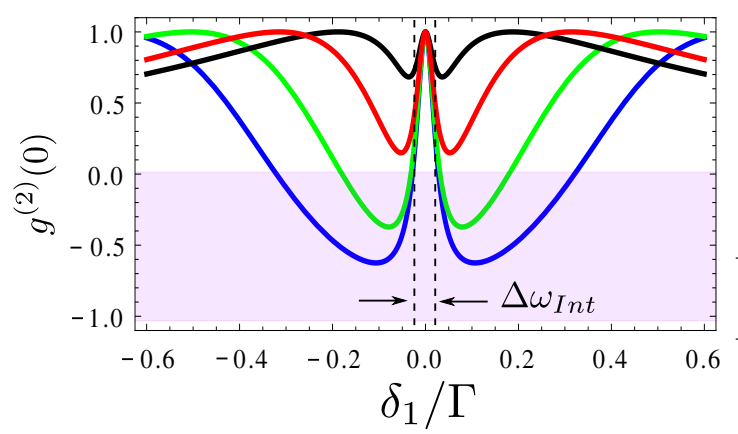

(a)

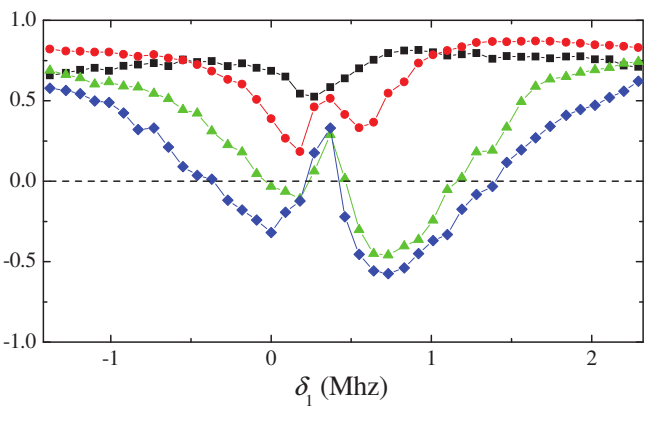

(b)

Figura 3.10: Resultado para a função de correlação $g^{(2)}(0)$ em função de dessintonia de um fóton, em [43]. (a) Teórico e (b) Experimental. Pela definição das dessintonias da figura 3.9.(a) e as definidas em [43], devemos considerar $\delta_{1} \equiv \Delta_{1}$.

Uma das características mais importantes deste modelo é o mapeamento entre a função 
de correlação e a absorção e dispersão do campo em interação com o meio. Para ilustrar tal mapeamento, a figura 3.11 mostra a função de correlação para os dois regimes mencionados anteriormente, junto com absorção e dispersão em cada caso. Na figura 3.11.(a), consideramos o caso em que $\Omega_{1}=0.3 \Gamma$, em que mapeamos a função de correlação com a absorção e a dispersão devida ao átomos para diferentes pontos de dessintonia. Para a dessintonia 1, a dispersão dos dois campos é nula, e a absorção toma valores próximos para os dois campos de modo que o produto $\operatorname{Im} \rho_{13} \operatorname{Im} \rho_{23}>0$ domina sobre a dispersão na eq. (3.35), induzindo assim correlação máxima $g^{(2)}(0)=1$. No ponto de dessintonia 2, podemos observar que a dispersão toma seu valor máximo para cada campo onde o produto $\operatorname{Re} p_{1} \operatorname{Re} p_{2}<0$, porém, ele não supera a contribuição positiva do produto das absorções $\operatorname{Im} p_{1} \operatorname{Im} p_{2}>0$, mantendo assim a correlação positiva. No ponto de dessintonia 3, a correlação aumenta de novo para um valor próximo de $g^{(2)}(0)=1$, já que a absorção é máxima e a dispersão novamente é nula. Finalmente no ponto de dessintonia 4, a absorção diminui tomando valores próximos aos da dispersão de um dos campo, diminuindo assim a correlação. Para as dessintonias $\delta<0$ a analise é a mesma.

Por outro lado, ao aumentar a intensidade dos campos, o mapeamento entre a função de correlação e os parâmetros atômicos apresentam características diferentes, como mostra a figura 3.11.(b). Da mesma forma que no caso anterior, tomamos diferentes pontos de dessintonia para analisar a relação $g^{(2)}(0) \longleftrightarrow \operatorname{Im} p_{i}, \operatorname{Re} p_{i}$. No ponto de transparência rotulado com 1, a situação se descreve de forma idêntica ao caso da figura 3.11.(a). No entanto, no ponto de dessintonia 2, ao contrário do caso na figura 3.11.(a), a dispersão toma valores tais que $\left|\operatorname{Re} p_{1} \operatorname{Re} p_{2}\right|>\left|\operatorname{Im} p_{1} \operatorname{Im} p_{2}\right|$ e assim, essa competição entre a dispersão e a absorção, faz com que a função de correlação tome valores negativos, ou seja, o meio induz uma anticorrelação entre os campos. Continuando com a dessintonia 3, a correlação é nula, porque o produto das dispersões se iguala ao produto das absorções. Nos pontos 4 e 5 , as dispersões dos dois campos tomam valores com o mesmo sinal, de modo que o produto $\operatorname{Re} p_{1} \operatorname{Re} p_{2}$ vai contribuir para que a correlação se mantenha em um regime $g^{(2)}(0)>0$. Para as dessintonias $\delta<0$ a análise é a mesma.

Uma outra forma de representar o mapeamento $g^{(2)}(0) \longleftrightarrow\left\{\operatorname{Im} p_{i}, \operatorname{Re} p_{i}\right\}$, é medi- 


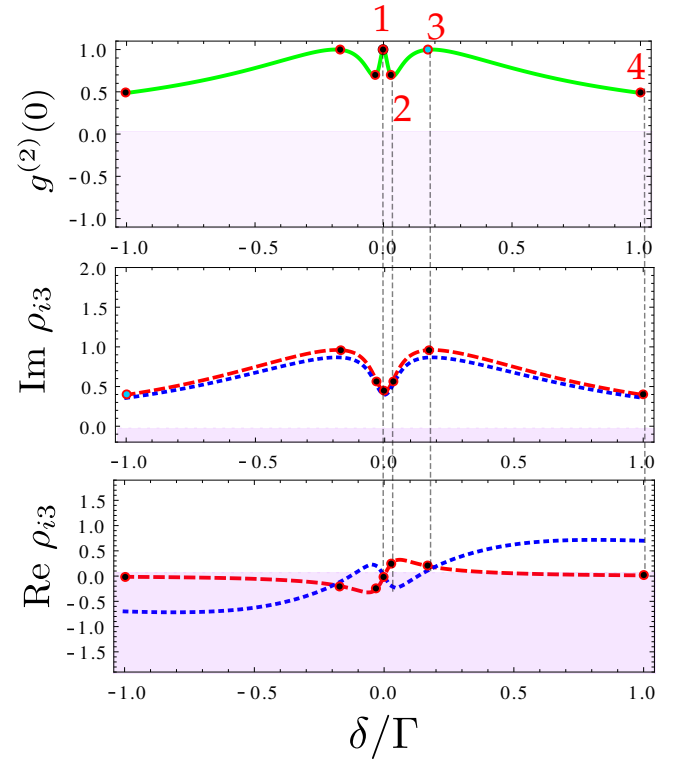

(a)

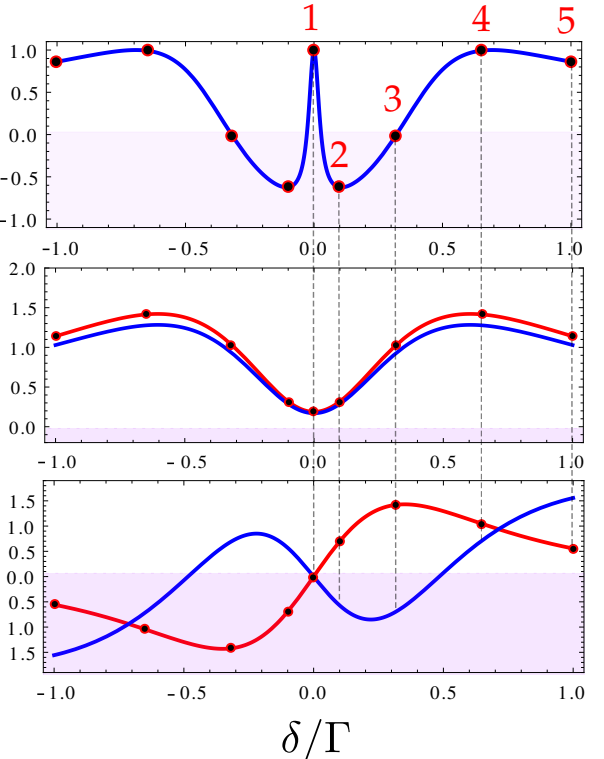

(b)

Figura 3.11: Mapeamento entre a função de correlação, a absorção e a dispersão dos campos para: (a) $\Omega_{1}=0.1 \Gamma$ onde os pontos de dessintonia analisados são: $1 \rightarrow \delta=0,2 \rightarrow \delta=0.03 \Gamma, 3 \rightarrow \delta=0.17 \Gamma$, $4 \rightarrow \delta=\Gamma$. (b) para $\Omega_{1}=0.4 \Gamma$ onde os pontos de dessintonia analisados são: $1 \rightarrow \delta=0$, $2 \rightarrow \delta=0.1 \Gamma, 3 \rightarrow \delta=0.32 \Gamma, 4 \rightarrow \delta=0.65 \Gamma$ e $4 \rightarrow \delta=\Gamma$. Parâmetros do cálculo: $\gamma / 2 \pi=150$ $k H z, \Delta_{2}=0$ e $\Delta_{1}=\delta$.

ante uma representação no plano complexo como mostra a figura 3.12. Aqui, a correlação e a anticorrelação estão relacionadas com a distância das trajetórias representadas pelas coerências $p_{1}$ e $p_{2}$. Para ilustrar esta interpretação, consideramos os casos de baixa e alta intensidade apresentados acima na figura 3.11. As figuras 3.12.(a-d) correspondem à função de correlação com frequência de Rabi $\Omega_{1}=0.4 \Gamma$, analisando cinco pontos de dessintonia da correlação com a sua respectiva representação no plano complexo. A figura (a) mostra o ponto de dessintonia 5 em que as coerências $p_{1}$ e $p_{2}$ estão representadas no plano complexo como um ponto azul e preto, respectivamente. A correlação, embora não seja a máxima, é positiva, o que se reflete em uma certa distância entre as coerências dentro do mesmo quadrante do plano complexo. Na figura (b) analisamos o ponto de dessintonia 4 para o qual a correlação toma um valor muito próximo da unidade, o que implica em uma distância mais próxima entre coerências, com respeito ao caso da figura (a). A figura (c), no entanto, mostra o ponto de dessintonia 3, para a qual a correlação é nula e a distância entre as coerências 
associadas a cada campo é máxima. A figura (d) mostra dois pontos de dessintonia: o ponto 2 em que temos anticorrelação entre os campos, o que se reflete em uma distância entre os campos, muito próxima à do caso da figura (a). No entanto, os pontos estão em quadrantes diferentes e, portanto, a correlação é negativa. Finalmente, no ponto de dessintonia 1 em que temos transparência, vemos que as coerências se superpõem, a distância entre os ponto é nula e a correlação é máxima $g^{(2)}(0)=1$. Continuando com as dessintonias $\delta<0$,

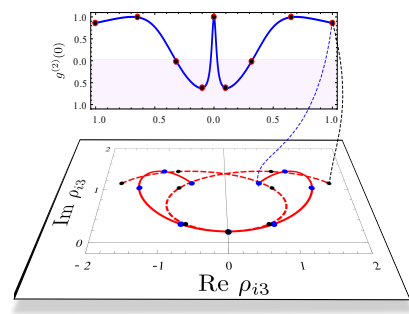

(a)

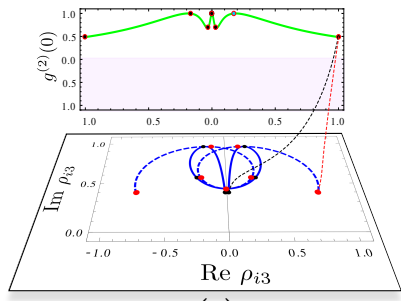

(e)

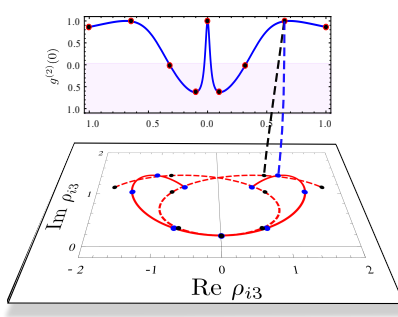

(b)

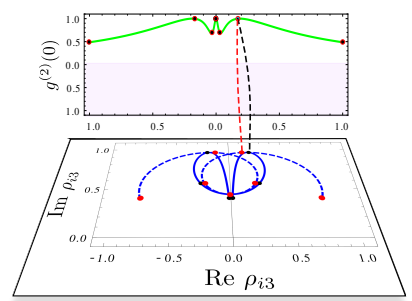

(f)

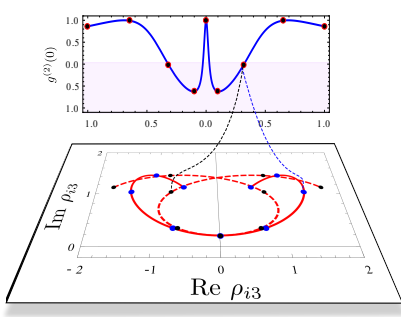

(c)

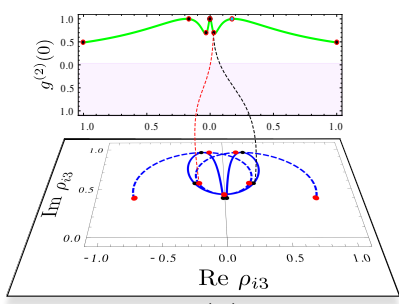

(g)

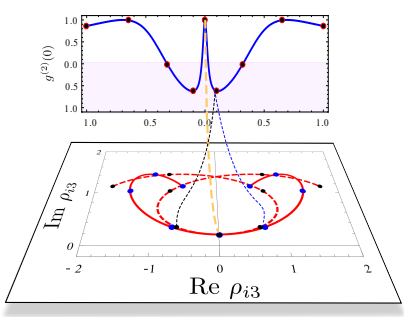

(c)

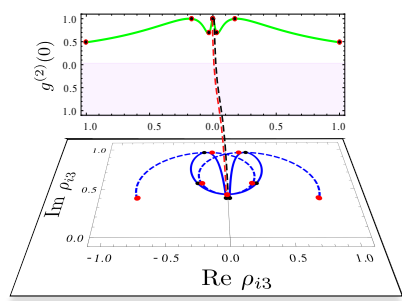

(h)

Figura 3.12: Mapeamento entre a correlação e a representação no plano complexo da absorção e a dispersão. Para $\Omega_{1}=0.4 \Gamma$ analisamos (a) $\delta=\Gamma,(b) \delta=0.65 \Gamma,(c) \delta=0.35 \Gamma e(d) \delta=0.1 \Gamma e$ $\delta=0$. Para $\Omega_{1}=0.1 \Gamma$ analisamos $(e) \delta=\Gamma,(f) \delta=0.17 \Gamma,(g) \delta=0.03 \Gamma e(h) \delta=0$. Esse pontos de dessintonia correspondem aos analisados na figura 3.11 .

no plano complexo continua-se traçando uma trajetória simétrica que apresenta as mesmas características acabadas de mencionar.

Agora analisaremos o caso em que temos um regime de baixa intensidade. As figuras 3.12.(e-h) mostram a função de correlação com frequência de Rabi $\Omega_{1}=0.1 \Gamma$ para os quatro pontos de dessintonia da figura 3.11.(b). A figura (e) mostra o caso de dessintonia 4, em que a correlação não é máxima e, portanto, existe uma distância entre as coerências atômicas representadas por pontos vermelho e preto. A figura (f) mostra o ponto de dessintonia 3, em que a distância entre as coerências é muito próxima, ou seja, as trajetórias se cruzam. A figura $(\mathrm{g})$ mostra o ponto de dessintonia 2 , em que a correlação diminui devido à distância 
que há entre as coerências. Vemos que o grau de correlação no ponto 4 é menor que no ponto 2, o que se reflete em uma distâncias maior entre as coerências para o ponto 4 que para do ponto 2. Por último, a figura (h) mostra que as trajetórias das coerências se superpõem devido à transparência induzida.

Assim vimos como o modelo heurístico permite, de forma simples, fazer um mapeamento entre a função de correlação $g^{(2)}(0)$ para dois campos em configuração $\Lambda$-EIT e as propriedades de absorção e dispersão associadas ao meio. Esta abordagem temporal da função de correlação, permite compará-la diretamente com os trabalhos experimentais em $[15,40,43,49]$. No entanto, a abordagem adotada no nosso experimento é no domínio da frequências, para a qual revisaremos o modelo de difusão de fase com um tratamento para variáveis estocásticas. Assim, como no domínio temporal se buscou estabelecer uma relação direta entre a correlação e as variáveis atômicas, no domínio das frequências também temos interesse em estabelecer uma relação desse tipo.

\subsection{Dinâmica do sistema átomo-campo com fase estocástica mediante cálculo de Itô}

Na seção 1.3.3 analisamos o exemplo em que temos um oscilador harmônico com fase estocástica, cuja dinâmica pode ser abordada mediante o calculo de Itô. Nesta seção queremos estudar a dinâmica dos átomos (os quais podem ser considerados como osciladores harmônicos) quando interagem com os campos de luz que apresentam flutuações de fase. Em particular, consideraremos a situação em que os campos interagem em condição de EIT, e faremos uma revisão da dinâmica do sistema átomo-campo empregando o cálculo de Itô descrito em [16], que tem sido uma abordagem adotada na pesquisa do LMCAL.

Consideramos novamente o sistema $\Lambda$-EIT da figura 3.9.(a), dessa vez descrevendo os 
campos como

$$
\mathbf{E}_{i}^{(+)}(t)=\mathcal{E}_{i} e^{i\left(\omega_{i} t+\phi_{i}(t)\right)} \mathbf{e}_{i} \quad i=1,2
$$

em que as fases $\phi_{1}(t)$ e $\phi_{2}(t)$ evoluem como processos de Wiener. Portanto, a média e a variância dessas variáveis são determinadas de acordo com as eqs. (1.47) e (1.48), tal que os seus elementos diferenciais satisfazem

$$
\begin{aligned}
\left\langle d \phi_{i}(t)\right\rangle & =0, \\
\left\langle d \phi_{i}(t), d \phi_{i}(t)\right\rangle & =2 \gamma_{i} d t, \\
\left\langle d \phi_{1}(t), d \phi_{2}(t)\right\rangle & =2 \gamma_{12} d t, \\
\left\langle d \phi_{i}(t)^{n}, d \phi_{j}(t)^{m}\right\rangle & =0 \text { para } n>1, m>1,
\end{aligned}
$$

onde a variância $\left\langle d \phi_{i}(t), d \phi_{j}(t)\right\rangle$ é idêntica ao segundo momento $\left\langle d \phi_{i}(t) d \phi_{j}(t)\right\rangle$, pois os valores médios desses elementos diferencias estocásticos são nulos.

Reescrevendo o hamiltoniano para o sistema $\Lambda$ de três níveis definido na eq.(2.15), considerando os campos da eq.(3.42), temos

$$
H_{\mathrm{int}}^{3 N}=\hbar\left(\Omega_{1}^{*} \hat{\sigma}_{13} e^{i\left(\omega_{1} t+\phi_{1}(t)\right)}+\Omega_{2}^{*} \hat{\sigma}_{23} e^{i\left(\omega_{2} t+\phi_{2}(t)\right)}+\mathrm{adj} .\right) .
$$

A partir da eq.(2.17) obtemos o conjunto de equações de Bloch para os elementos da matriz densidade, tal que

$$
\begin{aligned}
& \dot{\rho}_{11}=i \Omega_{1}^{*} \rho_{13} e^{-i\left(\omega_{1} t+\phi_{1}\right)}-i \Omega_{1} \rho_{31} e^{i\left(\omega_{1} t+\phi_{1}\right)}+\Gamma\left(1-\rho_{11}-\rho_{22}\right), \\
& \dot{\rho}_{22}=i \Omega_{2}^{*} \rho_{23} e^{-i\left(\omega_{2} t+\phi_{2}\right)}-i \Omega_{2} \rho_{32} e^{i\left(\omega_{2} t+\phi_{2}\right)}+\Gamma\left(1-\rho_{11}-\rho_{22}\right), \\
& \dot{\rho}_{13}=-\left(\Gamma+i \omega_{(31)}\right) \rho_{13}-i \Omega_{1}\left(1-2 \rho_{11}-\rho_{22}\right) e^{i\left(\omega_{1} t+\phi_{1}\right)}+i \Omega_{2} \rho_{12} e^{i\left(\omega_{2} t+\phi_{2}\right)}, \\
& \dot{\rho}_{32}=-\left(\Gamma-i \omega_{(32)}\right) \rho_{32}-i \Omega_{2}^{*}\left(1-2 \rho_{22}-\rho_{11}\right) e^{-i\left(\omega_{2} t+\phi_{2}\right)}+i \Omega_{1}^{*} \rho_{21} e^{-i\left(\omega_{1} t+\phi_{1}\right)}, \\
& \dot{\rho}_{12}=-\left(\gamma_{d}+\omega_{(31)}-\omega_{(32)}\right) \rho_{12}-i \Omega_{1} \rho_{32} e^{i\left(\omega_{1} t+\phi_{1}\right)}+i \Omega_{2}^{*} \rho_{13} e^{-i\left(\omega_{2} t+\phi_{2}\right)} .
\end{aligned}
$$


Este conjunto de equações podem ser reescritas de forma compacta mediante representação matricial no espaço de Liouville, como foi feito na seção anterior. Portanto, definimos os vetores $\mathbf{y}=\left\{\rho_{11}, \rho_{22}, \rho_{13}, \rho_{31}, \rho_{32}, \rho_{23}, \rho_{12}, \rho_{21}\right\}$ e $\mathbf{x}=\left\{\rho_{11}, \rho_{22}, \tilde{\rho}_{13}, \tilde{\rho}_{31}, \tilde{\rho}_{23}, \tilde{\rho}_{32}, \tilde{\rho}_{12}, \tilde{\rho}_{21}\right\}$ que satisfazem a relação de transformação

$$
\begin{aligned}
& \mathbf{y}(t)=e^{-i \mathbf{N}_{1}\left(\omega_{1} t+\phi_{1}(t)\right)} e^{-i \mathbf{N}_{2}\left(\omega_{2} t+\phi_{2}(t)\right)} \mathbf{x}(t) \\
& \mathbf{x}(t)=e^{i \mathbf{N}_{1}\left(\omega_{1} t+\phi_{1}(t)\right)} e^{i \mathbf{N}_{2}\left(\omega_{2} t+\phi_{2}(t)\right)} \mathbf{y}(t)
\end{aligned}
$$

em que as matrizes $\mathbf{N}_{1}$ e $\mathbf{N}_{2}$ são definidas por

$$
\mathbf{N}_{1}=\left[\begin{array}{cccccccc}
0 & 0 & 0 & 0 & 0 & 0 & 0 & 0 \\
0 & 0 & 0 & 0 & 0 & 0 & 0 & 0 \\
0 & 0 & -1 & 0 & 0 & 0 & 0 & 0 \\
0 & 0 & 0 & 1 & 0 & 0 & 0 & 0 \\
0 & 0 & 0 & 0 & 0 & 0 & 0 & 0 \\
0 & 0 & 0 & 0 & 0 & 0 & 0 & 0 \\
0 & 0 & 0 & 0 & 0 & 0 & -1 & 0 \\
0 & 0 & 0 & 0 & 0 & 0 & 0 & 1
\end{array}\right], \mathbf{N}_{2}=\left[\begin{array}{cccccccc}
0 & 0 & 0 & 0 & 0 & 0 & 0 & 0 \\
0 & 0 & 0 & 0 & 0 & 0 & 0 & 0 \\
0 & 0 & 0 & 0 & 0 & 0 & 0 & 0 \\
0 & 0 & 0 & 0 & 0 & 0 & 0 & 0 \\
0 & 0 & 0 & 0 & -1 & 0 & 0 & 0 \\
0 & 0 & 0 & 0 & 0 & 1 & 0 & 0 \\
0 & 0 & 0 & 0 & 0 & 0 & 1 & 0 \\
0 & 0 & 0 & 0 & 0 & 0 & 0 & -1
\end{array}\right]
$$

onde $\left[\mathbf{N}_{1}, \mathbf{N}_{2}\right]=0$, de modo que as eqs. (3.45)-(3.49) podem ser reescritas, em função dos vetores $\mathrm{y}$ e $\mathrm{x}$, da seguinte forma:

$$
\frac{d \mathbf{y}(t)}{d t}=-e^{-i \mathbf{N}_{1}\left(\omega_{1} t+\phi_{1}(t)\right)} e^{-i \mathbf{N}_{2}\left(\omega_{2} t+\phi_{2}(t)\right)} \mathcal{A} \mathbf{x}(t)+\mathbf{y}_{0},
$$

onde definimos a matriz $\mathcal{A}$.

$$
\mathcal{A}=\left(\begin{array}{cccccccc}
\Gamma & \Gamma & i \Omega_{1}^{*} & -i \Omega_{1} & 0 & 0 & 0 & 0 \\
\Gamma & \Gamma & 0 & 01 & i \Omega_{2}^{*} & -i \Omega_{2} & 0 & 0 \\
2 i \Omega_{1} & i \Omega_{1} & \left(\Gamma-i \omega_{(31)}\right) & 0 & 0 & 0 & i \Omega_{2} & 0 \\
-2 i \Omega_{1}^{*} & -i \Omega_{1}^{*} & 0 & \left(\Gamma+i \omega_{(31)}\right) & 0 & 0 & 0 & -i \Omega_{2}^{*} \\
i \Omega_{2} & 2 i \Omega_{2} & 0 & 0 & \left(\Gamma-i \omega_{(32)}\right) & 0 & 0 & i \Omega_{1}^{*} \\
-i \Omega_{2}^{*} & -2 i \Omega_{2}^{*} & 0 & 0 & 0 & \left(\Gamma+i \omega_{(32)}\right) & -i \Omega_{1} & 0 \\
0 & 0 & i \Omega_{2} & 0 & 0 & -i \Omega_{1}^{*} & \left(\gamma_{d}-i \omega_{(21)}\right) & 0 \\
0 & 0 & 0 & -i \Omega_{2}^{*} & i \Omega_{1} & 0 & 0 & \left(\gamma_{d}+i \omega_{(21)}\right)
\end{array}\right)
$$


e o vetor $\mathbf{y}_{0}$

$$
\mathbf{y}_{0}=\left(\Gamma, \Gamma,-i \Omega_{1} e^{i\left(\omega_{1} t+\phi_{1}\right)}, i \Omega_{1}^{*} e^{-i\left(\omega_{1} t+\phi_{1}\right)},-i \Omega_{2}^{*} e^{-i\left(\omega_{2} t+\phi_{2}\right)}, i \Omega_{2} e^{i\left(\omega_{2} t+\phi_{2}\right)}, 0,0\right),
$$

em que $\omega_{(i j)}$ corresponde à diferença de frequência entre os níveis atômicos $|i\rangle$ e $|j\rangle$, $\Omega_{1}$ e $\Omega_{2}$ às frequências de Rabi de cada um dos campos e $\gamma_{d}$ à decoerência entre os níveis fundamentais. Desta forma, temos definida a dinâmica dos elementos rapidamente variáveis $\mathbf{y}(t)$ em termos dos elementos lentamente variáveis $\mathbf{x}(t)$ da matriz densidade. Cabe notar que o formalismo matricial que descreve a eq.(3.53) poderia ser estendido para descrever qualquer sistema com $l$ níveis atômicos, $\operatorname{com} l>3$, e acoplados a dois campos que apresentam difusão de fase. Isto é possível simplesmente aumentado a dimensão dos vetores e as matrices que descrevem o sistema. No caso mais geral, poderíamos analisar um átomo de $l$ níveis atômicos acoplados com mais de dois campos com fase estocástica. Para isto, basta aumentar os termos na fase $\exp \left\{i \mathbf{N}_{1}\left(\omega_{1} t+\phi_{1}(t)\right)+\cdots+i \mathbf{N}_{n}\left(\omega_{n} t+\phi_{n}(t)\right)\right\}$ da eq.(3.53). No entanto, nesta seção nos limitaremos a um sistema de três níveis.

As equações de Bloch descritas pela eq.(3.53) são explicitamente dependentes do tempo. Uma dinâmica representada por um conjunto de equações implicitamente no tempo, é melhor descrita pelo vetor $\mathbf{x}(t)$. Essa dinâmica será então uma equação de Langevin, que descreve a evolução temporal do nosso sistema estocástico. De acordo com a eq.(1.63), ao aplicar a regra de diferenciação de Itô $d \mathbf{x}=\left[\partial_{t} \mathbf{x}+\frac{1}{2}\left(\partial_{\phi_{1}}^{2} \mathbf{x}+\partial_{\phi_{2}}^{2} \mathbf{x}+\partial_{\phi_{1} \phi_{2}}^{2} \mathbf{x}+\partial_{\phi_{2} \phi_{1}}^{2} \mathbf{x}\right)\right] d t+\partial_{\phi_{1}} \mathbf{x} d \phi_{1}+$ $\partial_{\phi_{2}} \mathbf{x} d \phi_{2}$ na eq. (3.51), obtemos então a seguinte equação de Langevin

$$
\begin{aligned}
d \mathbf{x}(t) & =\mathbf{a}[\mathbf{x}, t] d t+\mathbf{B}_{\mathbf{1}}[\mathbf{x}, t] d \phi_{1}(t)+\mathbf{B}_{\mathbf{2}}[\mathbf{x}, t] d \phi_{2}(t) \\
& -\frac{1}{2}\left(\mathbf{N}_{1}^{2} d \phi_{1}^{2}+\mathbf{N}_{2}^{2} d \phi_{2}^{2}+2 \mathbf{N}_{1} \mathbf{N}_{2} d \phi_{1} d \phi_{2}\right) \mathbf{x}(t)
\end{aligned}
$$


onde

$$
\begin{aligned}
\mathbf{a}[\mathbf{x}, t] & =-\mathbf{A}_{t} \mathbf{x}(t)+\mathbf{x}_{0}, \\
\mathbf{B}_{\mathbf{1}}[\mathbf{x}, t] & =i \mathbf{N}_{1} \mathbf{x}(t), \\
\mathbf{B}_{\mathbf{2}}[\mathbf{x}, t] & =i \mathbf{N}_{2} \mathbf{x}(t),
\end{aligned}
$$

com $\mathbf{A}_{t}=\mathcal{A}-i \mathbf{N}_{1} \omega_{1}-i \mathbf{N}_{2} \omega_{2}$ e $\mathbf{x}_{0}$ que corresponde à transformação do vetor $\mathbf{y}_{0}$ de acordo com a eq.(3.51). Note que a dinâmica descrita pela matriz $\mathbf{A}_{t}$ e $\mathbf{x}_{0}$ na eq.(3.56) corresponde à do sistema interagindo com campos monocromáticos sem flutuação de fase. Entretanto, os termos proporcionais às matrices $\mathbf{N}_{i}$ revelam a contribuição das flutuações de fase $d \phi_{i}$. Como foi revisado na seção 1.3, a principal característica deste tipo de evolução estocástica é que, diferente do movimento Browniano, em que as flutuações estocásticas entram no sistema de forma aditiva, neste caso, as flutuações de fase são amplificadas pelas variáveis atômicas devido ao produto $d \phi_{i} \times \mathbf{x}(t)$ que retro-alimentam a evolução do sistema.

Dada a equação de Langevin que descreve o nosso sistema, em seguida calculamos o valor médio das variáveis atômicas. De acordo com as eqs.(3.43a)-(3.43c), o valor médio e a variância das flutuações de fase são $\left\langle d \phi_{i}\right\rangle=0$ e $\left\langle d \phi_{i}^{2}\right\rangle=2 \gamma_{i}$, de modo que

$$
d\langle\mathbf{x}(t)\rangle=-\mathbf{M}\langle\mathbf{x}(t)\rangle+\mathbf{x}_{0} d t
$$

onde definimos

$$
\mathbf{M}=\left[\gamma_{1} \mathbf{N}_{1}^{2}+\gamma_{2} \mathbf{N}_{2}^{2}+2 \gamma_{12} \mathbf{N}_{1} \mathbf{N}_{2}+\mathbf{A}_{t}\right]
$$

Podemos notar que esta é uma equação linear equivalente à da eq.(3.39), obtida na seção anterior. Portanto, a solução estacionária do valor médio é dada por

$$
\langle\mathbf{x}(t)\rangle_{e s}=\mathbf{M}^{-1} \mathbf{x}_{0}=\left(\tilde{\rho}_{11}^{e s}, \tilde{\rho}_{22}^{e s}, \tilde{\rho}_{13}^{e s}, \tilde{\rho}_{31}^{e s}, \tilde{\rho}_{23}^{e s}, \tilde{\rho}_{32}^{e s}, \tilde{\rho}_{12}^{e s}, \tilde{\rho}_{21}^{e s}\right)
$$

A partir desta solução podemos obter os elementos $p_{1}=\tilde{\rho}_{13}^{e s}$ e $p_{2}=\tilde{\rho}_{32}^{e s}$, com os quais podemos determinar a função de correlação $g^{(2)}(0)$ aplicando o modelo heurístico da seção 
anterior. No entanto, para determinar de maneira formal a função de correlação, levando em conta a influência do processo de difusão de fase na dinâmica atômica, é necessário calcular a matriz de covariância dos elementos atômicos a partir da solução da eq.(3.56).

\subsubsection{Matriz de Covariância das variáveis atômicas}

A matriz de covariância das variáveis atômicas é definida como

$$
\begin{aligned}
\tilde{\mathbf{g}}(\tau)=\left\langle\mathbf{x}(t+\tau), \mathbf{x}(t)^{\dagger}\right\rangle & \equiv\left\langle[\mathbf{x}(t+\tau)-\langle\mathbf{x}(t+\tau)\rangle][\mathbf{x}(t)-\langle\mathbf{x}(t)\rangle]^{\dagger}\right\rangle \\
& =\left\langle\mathbf{x}(t+\tau) \mathbf{x}(t)^{\dagger}\right\rangle-\langle\mathbf{x}(t+\tau)\rangle\left\langle\mathbf{x}(t)^{\dagger}\right\rangle
\end{aligned}
$$

e representada por

$$
\tilde{\mathbf{g}}(\tau)=\left(\begin{array}{cccccccc}
\left\langle\tilde{\rho}_{11}(t+\tau), \tilde{\rho}_{11}(t)\right\rangle & \ldots & \left\langle\tilde{\rho}_{11}(t+\tau), \tilde{\rho}_{31}(t)\right\rangle & \left\langle\tilde{\rho}_{11}(t+\tau), \tilde{\rho}_{13}(t)\right\rangle & \left\langle\tilde{\rho}_{11}(t+\tau), \tilde{\rho}_{32}(t)\right\rangle & \left\langle\tilde{\rho}_{11}(t+\tau), \tilde{\rho}_{23}(t)\right\rangle & \ldots & \ldots \\
\left\langle\tilde{\rho}_{11}(t+\tau), \tilde{\rho}_{22}(t)\right\rangle & \ldots & \left\langle\tilde{\rho}_{22}(t+\tau), \tilde{\rho}_{31}(t)\right\rangle & \left\langle\tilde{\rho}_{22}(t+\tau), \tilde{\rho}_{13}(t)\right\rangle & \left\langle\tilde{\rho}_{22}(t+\tau), \tilde{\rho}_{32}(t)\right\rangle & \left\langle\tilde{\rho}_{22}(t+\tau), \tilde{\rho}_{23}(t)\right\rangle & \ldots & \ldots \\
\left\langle\tilde{\rho}_{11}(t+\tau), \tilde{\rho}_{31}(t)\right\rangle & \ldots & \left\langle\tilde{\rho}_{13}(t+\tau), \tilde{\rho}_{31}(t)\right\rangle & \left\langle\tilde{\rho}_{13}(t+\tau), \tilde{\rho}_{13}(t)\right\rangle & \left\langle\tilde{\rho}_{13}(t+\tau), \tilde{\rho}_{32}(t)\right\rangle & \left\langle\tilde{\rho}_{13}(t+\tau), \tilde{\rho}_{23}(t)\right\rangle & \ldots & \ldots \\
\vdots & \vdots & \left\langle\tilde{\rho}_{31}(t+\tau), \tilde{\rho}_{31}(t)\right\rangle & \left\langle\tilde{\rho}_{31}(t+\tau), \tilde{\rho}_{13}(t)\right\rangle & \left\langle\tilde{\rho}_{31}(t+\tau), \tilde{\rho}_{32}(t)\right\rangle & \left\langle\tilde{\rho}_{31}(t+\tau), \tilde{\rho}_{23}(t)\right\rangle & \vdots & \vdots \\
\vdots & \vdots & \left\langle\tilde{\rho}_{23}(t+\tau), \tilde{\rho}_{31}(t)\right\rangle & \left\langle\tilde{\rho}_{23}(t+\tau), \tilde{\rho}_{13}(t)\right\rangle & \left\langle\tilde{\rho}_{23}(t+\tau), \tilde{\rho}_{32}(t)\right\rangle & \left\langle\tilde{\rho}_{23}(t+\tau), \tilde{\rho}_{23}(t)\right\rangle & \vdots & \vdots \\
\vdots & \vdots & \left\langle\tilde{\rho}_{32}(t+\tau), \tilde{\rho}_{31}(t)\right\rangle & \left\langle\tilde{\rho}_{32}(t+\tau), \tilde{\rho}_{13}(t)\right\rangle & \left\langle\tilde{\rho}_{32}(t+\tau), \tilde{\rho}_{32}(t)\right\rangle & \left\langle\tilde{\rho}_{32}(t+\tau), \tilde{\rho}_{23}(t)\right\rangle & \vdots & \vdots \\
\vdots & \vdots & \left\langle\tilde{\rho}_{12}(t+\tau), \tilde{\rho}_{31}(t)\right\rangle & \left\langle\tilde{\rho}_{12}(t+\tau), \tilde{\rho}_{13}(t)\right\rangle & \left\langle\tilde{\rho}_{12}(t+\tau), \tilde{\rho}_{32}(t)\right\rangle & \left\langle\tilde{\rho}_{12}(t+\tau), \tilde{\rho}_{23}(t)\right\rangle & \vdots & \vdots \\
\vdots & \vdots & \left\langle\tilde{\rho}_{21}(t+\tau), \tilde{\rho}_{31}(t)\right\rangle & \left\langle\tilde{\rho}_{21}(t+\tau), \tilde{\rho}_{13}(t)\right\rangle & \left\langle\tilde{\rho}_{21}(t+\tau), \tilde{\rho}_{32}(t)\right\rangle & \left\langle\tilde{\rho}_{21}(t+\tau), \tilde{\rho}_{23}(t)\right\rangle & \vdots & \vdots
\end{array}\right) .
$$

Nela estão contidos os elementos necessários para, mais adiante, determinar a função de correlação $g^{(2)}(\tau)$ definida na eq.(1.22). A matriz de covariância descreve então as flutuações dos elementos atômicos $x_{i}(t+\tau)$, separados por um intervalo de tempo $\tau$, com referência ao elementos $x_{j}(t)$ definidos no tempo $t$. Portanto, para analisar a dinâmica da matriz de covariância, precisamos analisar as duas escalas de tempo: a dinâmica que descreve as mudanças com relação à defasagem temporal $\tau$ e dinâmica relacionada com o tempo de referência $t$.

Consideremos primeiro a dinâmica relacionada ao intervalo de tempo $\tau \neq 0$. Neste caso, 
podemos empregar o teorema da regressão [80], tal que para $\tau>0$ temos

$$
\begin{aligned}
\frac{d \tilde{\mathbf{g}}(\tau)}{d \tau} d \tau= & \left\langle d \mathbf{x}(t+\tau), \mathbf{x}(t)^{\dagger}\right\rangle \\
= & \left\langle\left[-\mathbf{A}_{t} \mathbf{x}(t+\tau)+\mathbf{x}_{0}\right] d \tau+i\left[\mathbf{N}_{1} d \phi_{1}(t+\tau)+\mathbf{N}_{2} d \phi_{2}(t+\tau)\right] \mathbf{x}(t+\tau), \mathbf{x}(t)^{\dagger}\right\rangle \\
& -\frac{1}{2}\left\langle\left[\mathbf{N}_{1}^{2} d \phi_{1}(\tau)^{2}+\mathbf{N}_{2}^{2} d \phi_{2}(\tau)^{2}+2 \mathbf{N}_{1} \mathbf{N}_{2} d \phi_{1}(\tau) d \phi_{2}(\tau)\right] \mathbf{x}(t+\tau), \mathbf{x}(t)^{\dagger}\right\rangle
\end{aligned}
$$

Levando em conta que as variáveis $\mathbf{x}(\tau)$ são variáveis não antecipantes, e que as fases estocásticas satisfazem as eq.(3.43a)-(3.43c), então a eq.(3.64) se reduz a

$$
\begin{aligned}
d \tilde{\mathbf{g}}(\tau) & =\left[-\mathbf{M}\left\langle\mathbf{x}(t+\tau), \mathbf{x}(t)^{\dagger}\right\rangle+\left\langle\mathbf{x}_{0}, \mathbf{x}(t)^{\dagger}\right\rangle\right] d \tau \\
& =-\mathbf{M} \tilde{\mathbf{g}}(\tau) d \tau
\end{aligned}
$$

onde levamos em conta que a covariância do vetor constante $\mathbf{x}_{o}$ é nula. Da mesma forma se obtêm a dinâmica para intervalos de tempo $\tau<0$. Portanto, a dinâmica da matriz de covariância com relação à diferença temporal $\tau$ é dada por

$$
\begin{aligned}
\frac{d \tilde{\mathbf{g}}(\tau)}{d \tau}=-\mathbf{M} \tilde{\mathbf{g}}(\tau) & \\
\frac{d \tilde{\mathbf{g}}(\tau)}{d \tau}=\tilde{\mathbf{g}}(\tau) \mathbf{M}^{\dagger} & \tau<0
\end{aligned}
$$

Consequentemente, a sua solução com respeito à $\tau$ é

$$
\tilde{\mathbf{g}}(\tau)= \begin{cases}e^{-\mathbf{M} \tau} \tilde{\mathbf{g}}(0) & \tau>0 \\ \tilde{\mathbf{g}}(0) e^{\mathbf{M}^{\dagger} \tau} & \tau<0\end{cases}
$$

Esta solução precisa, portanto, da solução da matriz de covariância $\tilde{\mathbf{g}}(0)$ para um tempo ini$\operatorname{cial} \tau=0$. Com ela podemos determinar por completo a solução da matriz $\tilde{\mathbf{g}}(\tau)$. Isto requere uma análise da evolução temporal com relação a tempo $t$, dado que $\tilde{\mathbf{g}}(0)=\left\langle\mathbf{x}(t), \mathbf{x}(t)^{\dagger}\right\rangle$. Para isto, vamos construir a equação de Langevin para os elementos $\mathbf{c}(t, t)=\mathbf{x}(t) \mathbf{x}(t)^{\dagger}$, que fazem parte da eq.(3.62), aplicando a regra de diferenciação de Itô da eq.(1.63), da mesma forma 
que foi feito na eq.(3.56), tal que

$$
d \mathbf{c}(t, t)=\left(\frac{\partial \mathbf{c}}{\partial t}+\gamma_{1} \frac{\partial^{2} \mathbf{c}}{\partial \phi_{1}^{2}}+\gamma_{2} \frac{\partial^{2} \mathbf{c}}{\partial \phi_{2}^{2}}+\gamma_{12} \frac{\partial^{2} \mathbf{c}}{\partial \phi_{1} \partial \phi_{2}}\right) d t+\frac{\partial \mathbf{c}}{\partial \phi_{1}} d \phi_{1}(t)+\frac{\partial \mathbf{c}}{\partial \phi_{2}} d \phi_{2}(t)
$$

O primeiro termo da parte direita corresponde à variação explícita no tempo. Ele é determinado a partir da matriz $\mathbf{a}[\mathbf{x}, t]$ da eq.(3.56), tal que

$$
\begin{aligned}
\frac{\partial \mathbf{c}(t, t)}{\partial t} & =\left(\frac{\partial \mathbf{x}(t)}{\partial t}\right) \mathbf{x}^{\dagger}(t)+\mathbf{x}(t)\left(\frac{\partial \mathbf{x}^{\dagger}(t)}{\partial t}\right) \\
& =\mathbf{a}[\mathbf{x}, t] \mathbf{x}^{\dagger}(t)+\mathbf{x}(t) \mathbf{a}[\mathbf{x}, t]^{\dagger} \\
& =-\left[\mathbf{A}_{t} \mathbf{c}(t, t)+\mathbf{c}(t, t) \mathbf{A}_{t}^{\dagger}\right]+\mathbf{x}(t)^{\dagger} \mathbf{x}_{0}+\mathbf{x}(t) \mathbf{x}_{0}^{\dagger}
\end{aligned}
$$

Entretanto, para calcular as derivadas com respeito às fases $\phi_{i}$, expressamos a matriz $\mathbf{c}(t, t)$ explicitamente em termos das fases mediante a transformação (3.51), tal que

$$
\mathbf{c}(t, t)=\mathbf{x}(t) \mathbf{x}(t)^{\dagger}=e^{i \mathbf{N}_{1}\left(\omega_{1} t+\phi_{1}(t)\right)} e^{i \mathbf{N}_{2}\left(\omega_{2} t+\phi_{2}(t)\right)} \mathbf{y}(t) \mathbf{y}(t)^{\dagger} e^{-i \mathbf{N}_{1}\left(\omega_{1} t+\phi_{1}(t)\right)} e^{-i \mathbf{N}_{2}\left(\omega_{2} t+\phi_{2}(t)\right)} .
$$

Assim, as derivadas parciais com respeito às fases são dadas por

$$
\begin{aligned}
\frac{\partial \mathbf{c}(t, t)}{\partial \phi_{i}} & =i \mathbf{N}_{i} \mathbf{c}(t, t)-i \mathbf{c}(t, t) \mathbf{N}_{i}, \\
\frac{\partial^{2} \mathbf{c}(t, t)}{\partial \phi_{i}^{2}} & =-\mathbf{N}_{i}^{2} \mathbf{c}(t, t)-\mathbf{c}(t, t) \mathbf{N}_{i}^{2}+2 \mathbf{N}_{i} \mathbf{c}(t, t) \mathbf{N}_{i}, \\
\frac{\partial^{2} \mathbf{c}(t, t)}{\partial \phi_{i} \partial \phi_{j}} & =-\mathbf{N}_{i} \mathbf{N}_{j} \mathbf{c}(t, t)-\mathbf{c}(t, t) \mathbf{N}_{i} \mathbf{N}_{j}+2 \mathbf{N}_{i} \mathbf{c}(t, t) \mathbf{N}_{j} .
\end{aligned}
$$

Substituindo a eq.(3.70) e as eqs.(3.72)-(3.74) na equação diferencial (3.69), podemos então obter a dinâmica para o valor médio $\langle\mathbf{c}(t, t)\rangle$, tal que

$$
\begin{aligned}
\frac{d\langle\mathbf{c}(t, t)\rangle}{d t}= & -\left[\mathbf{M}\langle\mathbf{c}(t, t)\rangle+\langle\mathbf{c}(t, t)\rangle \mathbf{M}^{\dagger}\right]+\left[\mathbf{x}_{0}\left\langle\mathbf{x}(t)^{\dagger}\right\rangle+\langle\mathbf{x}(t)\rangle \mathbf{x}_{0}^{\dagger}\right] \\
& +2 \gamma_{1} \mathbf{N}_{1}\langle\mathbf{c}(t, t)\rangle \mathbf{N}_{1}+2 \gamma_{2} \mathbf{N}_{2}\langle\mathbf{c}(t, t)\rangle \mathbf{N}_{2}+2 \gamma_{12} \mathbf{N}_{1}\langle\mathbf{c}(t, t)\rangle \mathbf{N}_{2} \\
& +2 \gamma_{12} \mathbf{N}_{1}\langle\mathbf{c}(t, t)\rangle \mathbf{N}_{2},
\end{aligned}
$$


em que consideramos $\left\langle\partial_{\phi} \mathbf{c}(t, t) d \phi_{i}\right\rangle=0$, dado que $\mathbf{c}(t, t)$ é uma função não antecipante.

Voltando para a matriz de covariância, substituímos então a expressão $\langle\mathbf{c}(t, t)\rangle=\tilde{\mathbf{g}}(0)+$ $\langle\mathbf{x}(t)\rangle\left\langle\mathbf{x}(t)^{\dagger}\right\rangle$ da eq.(3.62) na eq.(3.75), e assim obtemos a dinâmica da matriz de covariância

$$
\begin{aligned}
\frac{d \tilde{\mathbf{g}}(0)}{d t}= & -\left[\mathbf{M} \tilde{\mathbf{g}}(0)+\tilde{\mathbf{g}}(0) \mathbf{M}^{\dagger}\right] \\
& +2 \gamma_{1} \mathbf{N}_{1} \tilde{\mathbf{g}}(0) \mathbf{N}_{1}+2 \gamma_{2} \mathbf{N}_{2} \tilde{\mathbf{g}}(0) \mathbf{N}_{2}+2 \gamma_{12} \mathbf{N}_{1} \tilde{\mathbf{g}}(0) \mathbf{N}_{2} \\
& +2 \gamma_{12} \mathbf{N}_{2} \tilde{\mathbf{g}}(0) \mathbf{N}_{1} \\
& +2 \gamma_{1} \mathbf{N}_{1}\langle\mathbf{x}(t)\rangle\left\langle\mathbf{x}(t)^{\dagger}\right\rangle \mathbf{N}_{1}+2 \gamma_{2} \mathbf{N}_{2}\langle\mathbf{x}(t)\rangle\left\langle\mathbf{x}(t)^{\dagger}\right\rangle \mathbf{N}_{2} \\
& +2 \gamma_{12} \mathbf{N}_{1}\langle\mathbf{x}(t)\rangle\left\langle\mathbf{x}(t)^{\dagger}\right\rangle \mathbf{N}_{2}+2 \gamma_{12} \mathbf{N}_{2}\langle\mathbf{x}(t)\rangle\left\langle\mathbf{x}(t)^{\dagger}\right\rangle \mathbf{N}_{1}
\end{aligned}
$$

Se considerarmos a situação experimental em que os campos são gerados pelo mesmo laser, então podemos assumir que $\gamma_{1}=\gamma_{2}=\gamma_{12}=\bar{\gamma}$ e, portanto, a dinâmica é simplificada da seguinte forma:

$$
\begin{aligned}
\frac{d \tilde{\mathbf{g}}(0)}{d t} & =-\left[\mathbf{M} \tilde{\mathbf{g}}(0)+\tilde{\mathbf{g}}(0) \mathbf{M}^{\dagger}\right]+2 \bar{\gamma} \Phi \tilde{\mathbf{g}}(0) \Phi \\
& +2 \bar{\gamma} \Phi\langle\mathbf{x}(t)\rangle\left\langle\mathbf{x}(t)^{\dagger}\right\rangle \Phi,
\end{aligned}
$$

onde $\Phi=\left(\mathbf{N}_{1}+\mathbf{N}_{2}\right)$. Assim, a solução estacionária $\tilde{\mathbf{g}}(0)_{e s}$ se obtém a partir da condição $d \tilde{\mathbf{g}}(0)=0$, tal que

$$
2 \bar{\gamma} \Phi\langle\mathbf{x}(t)\rangle_{e s}\left\langle\mathbf{x}(t)^{\dagger}\right\rangle_{e s} \Phi=\left[\mathbf{M} \tilde{\mathbf{g}}(0)_{e s}+\tilde{\mathbf{g}}(0)_{e s} \mathbf{M}^{\dagger}\right]-2 \bar{\gamma} \Phi \tilde{\mathrm{g}}(0)_{e s} \Phi .
$$

Substituindo a solução do valor médio $\langle\mathbf{x}(t)\rangle_{e s}$ da eq. (3.60) no sistema linear (3.78), podemos então determinar a solução estacionária da matriz de covariância ${ }^{12} \tilde{\mathbf{g}}(0)_{e s}$. Com ela determinamos também, de forma completa, a dinâmica em condição estacionária da matriz de covariância $\tilde{\mathbf{g}}(\tau)_{e s}$ de acordo com a solução (3.68).

\footnotetext{
${ }^{12}$ Para solucionar o sistema linear aplicamos um isomorfismo à eq.(3.78). Para isso mapeamos as componentes das matrizes $\tilde{\mathbf{g}}(0)_{e s} \mathrm{e}\langle\mathbf{x}(t)\rangle_{e s}\left\langle\mathbf{x}(t)^{\dagger}\right\rangle_{e s}$ em vetores no espaço de Liouville. Entretanto, as matrizes $\mathbf{M}, \mathbf{N}_{1} \mathrm{e}$ $\mathbf{N}_{2}$, que operam à esquerda ou à direta, são mapeadas em matrizes que operam no espaço de Lioville. Assim, podemos resolver o sistema de equações lineares no espaço de Liouville.
} 


\subsubsection{Covariância das intensidades dos campos}

A matriz de covariância $\tilde{\mathrm{g}}(\tau)_{\text {es }}$ contém todas as variáveis atômicas que vão permitir determinar a função de correlação $g^{(2)}(\tau)$ entre as intensidade dos campos. Para isso, consideramos a eq. (3.30), que descreve a intensidade dos campos $I_{i}(t)$ após a propagação no meio atômico. Portanto, a covariância das intensidades dos campos é escrita como

$$
\begin{aligned}
\left\langle I_{i}(t+\tau), I_{j}(t)\right\rangle & \equiv\left\langle I_{i}(t+\tau) I_{j}(t)\right\rangle-\left\langle I_{i}(t+\tau)\right\rangle\left\langle I_{j}(t)\right\rangle \\
& =\left\langle\left[\left|\tilde{\mathcal{E}}_{i}\right|^{2}+i \kappa_{i} \tilde{\rho}_{i 3}(t+\tau) \tilde{\mathcal{E}}_{i}-i \kappa_{i} \tilde{\rho}_{3 i}(t+\tau) \tilde{\mathcal{E}}_{i}\right],\left[\left|\tilde{\mathcal{E}}_{i}\right|^{2}+i \kappa_{j} \tilde{\rho}_{j 3}(t) \tilde{\mathcal{E}}_{j}-i \kappa_{i} \tilde{\rho}_{3 j}(t) \tilde{\mathcal{E}}_{j}\right]\right\rangle \\
& =\left\langle\left[i \kappa_{i} \tilde{\rho}_{i 3}(t+\tau) \tilde{\mathcal{E}}_{i}-i \kappa_{i} \tilde{\rho}_{3 i}(t+\tau) \tilde{\mathcal{E}}_{i}\right],\left[i \kappa_{j} \tilde{\rho}_{j 3}(t) \tilde{\mathcal{E}}_{j}-i \kappa_{i} \tilde{\rho}_{3 j}(t) \tilde{\mathcal{E}}_{j}\right] .\right\rangle
\end{aligned}
$$

Assumindo que as constantes de acoplamento satisfazem $\kappa_{i} \approx \kappa_{j}=\kappa$, o que é compatível às condições experimentais, então

$$
\begin{aligned}
\left\langle I_{i}(t+\tau), I_{j}(t)\right\rangle= & \mu_{i j}\left[\left\langle\tilde{\rho}_{i 3}(t+\tau), \tilde{\rho}_{j 3}(t)\right\rangle-\left\langle\tilde{\rho}_{i 3}(t+\tau), \tilde{\rho}_{3 j}(t)\right\rangle\right. \\
& \left.-\left\langle\tilde{\rho}_{3 i}(t+\tau), \tilde{\rho}_{j 3}(t)\right\rangle+\left\langle\tilde{\rho}_{3 i}(t+\tau), \tilde{\rho}_{3 j}(t)\right\rangle\right],
\end{aligned}
$$

onde $\mu_{i j}=\kappa^{2} \tilde{\mathcal{E}}_{i} \tilde{\mathcal{E}}_{j}$ e em que os termos $\left\langle\tilde{\rho}_{i 3}(t+\tau), \tilde{\rho}_{j 3}(t)\right\rangle$ e a suas permutações são elementos da matriz de covariância da eq.(3.63). Portanto, estas covariâncias podem ser reescritas em termos dos elementos da matriz $\tilde{\mathbf{g}}(\tau)$ como

$$
\begin{aligned}
& \left\langle I_{1}(t+\tau), I_{1}(t)\right\rangle=\mu_{11}\left[[\tilde{\mathbf{g}}(\tau)]_{34}+[\tilde{\mathbf{g}}(\tau)]_{43}-[\tilde{\mathbf{g}}(\tau)]_{33}-[\tilde{\mathbf{g}}(\tau)]_{44}\right] \\
& \left\langle I_{2}(t+\tau), I_{2}(t)\right\rangle=\mu_{22}\left[[\tilde{\mathbf{g}}(\tau)]_{56}+[\tilde{\mathbf{g}}(\tau)]_{65}-[\tilde{\mathbf{g}}(\tau)]_{55}-[\tilde{\mathbf{g}}(\tau)]_{66}\right] \\
& \left\langle I_{1}(t+\tau), I_{2}(t)\right\rangle=\mu_{11}\left[[\tilde{\mathbf{g}}(\tau)]_{36}+[\tilde{\mathbf{g}}(\tau)]_{43}-[\tilde{\mathbf{g}}(\tau)]_{35}-[\tilde{\mathbf{g}}(\tau)]_{45}\right]
\end{aligned}
$$

A partir da solução estacionária da matriz de covariância da eq.(3.78), podemos então determinar o conjunto de covariâncias das eqs.(3.81)-(3.83). Uma vez calculadas, podemos obter 
a função de correlação normalizada

$$
g^{(2)}(\tau)=\frac{\left\langle I_{1}(t+\tau), I_{2}(t)\right\rangle}{\sqrt{\left\langle I_{1}(t+\tau), I_{1}(t)\right\rangle\left\langle I_{2}(t+\tau), I_{2}(t)\right\rangle}} .
$$

Cabe notar que ao linearizar a intensidade $I_{i}=\left\langle I_{i}\right\rangle+\delta I_{i}$, onde $\left\langle\delta I_{i}\right\rangle=0$, a covariância satisfaz a relação $\left\langle I_{i}(t+\tau), I_{j}(t)\right\rangle=\left\langle\left\langle I_{i}\right\rangle+\delta I_{i}(t+\tau),\left\langle I_{j}\right\rangle+\delta I_{j}(t)\right\rangle=\left\langle\delta I_{i}(t+\tau) \delta I_{j}(t)\right\rangle$. Portanto, a correlação da eq.(3.84) é equivalente à que tínhamos definido na eq.(3.23).

Esta é então a descrição no domínio do tempo para determinar a função de correlação entre as intensidades dos campos com excesso de ruído de fase, acoplando um sistema de três níveis em condição de EIT. A seguir, mostraremos como é possível fazer a descrição no domínio das frequências, determinando a matriz de densidade espectral mediante a transformada de Fourier da matriz de covariância $\tilde{\mathbf{g}}(\tau)$.

\subsubsection{Matriz espectral S( $\omega)$ : Solução de Itô}

Como tem sido descrito ao longo deste capítulo, é do nosso interesse analisar a correlação de intensidades no domínio das frequências, pois o nosso sistema de medição no laboratório está adaptado para isto. Então é necessário passar de uma descrição temporal, como a da seção anterior, para uma descrição no domínio das frequências. Para isto, empregamos o teorema de Wiener-Khinthchine, aplicando a transformada de Fourier na matriz de covariância da eq.(3.68), de modo que a matriz de densidade espectral é

$$
\begin{aligned}
\mathbf{S}(\omega) & =\frac{1}{2 \pi}\left[\int_{-\infty}^{0} d \tau \tilde{\mathbf{g}}(\tau)_{e s} e^{i \omega \tau}+\int_{0}^{\infty} d \tau \tilde{\mathbf{g}}(\tau)_{e s} e^{i \omega \tau}\right] \\
& =\frac{1}{2 \pi}\left[\int_{-\infty}^{0} d \tau \tilde{\mathbf{g}}(0)_{e s} e^{\left(i \omega+\mathbf{M}^{\dagger}\right) \tau}+\int_{0}^{\infty} d \tau e^{(i \omega-\mathbf{M}) \tau} \tilde{\mathbf{g}}(0)_{e s}\right] \\
& =\frac{1}{2 \pi}\left[(\mathbf{M}-i \omega)^{-1} \tilde{\mathbf{g}}(0)_{e s}+\tilde{\mathbf{g}}(0)_{e s}\left(i \omega+\mathbf{M}^{\dagger}\right)^{-1}\right]
\end{aligned}
$$


Este resultado para a matriz espectral corresponde a de um processo de Ornstein-Uhlenbeck, que se caracteriza por ser um processo estocástico convergente ${ }^{13}$. Se calcularmos $[\mathbf{M}-$ $i \omega] \mathbf{S}(\omega)\left[\mathbf{M}^{\dagger}+i \omega\right]$, pela eq.(3.78), temos finalmente que a matriz de densidade espectral é dada por

$$
\mathbf{S}(\omega)=\frac{1}{\pi}\left[(\mathbf{M}-i \omega)^{-1} \Phi \Pi \Phi\left(\mathbf{M}^{\dagger}+i \omega\right)^{-1}\right]
$$

onde

$$
\Pi=\bar{\gamma}\left[\langle\mathbf{x}(t)\rangle_{e s}\left\langle\mathbf{x}(t)^{\dagger}\right\rangle_{e s}+\tilde{\mathbf{g}}(0)_{e s}\right]
$$

Assim, a partir da solução estacionária para $\langle\mathbf{x}(t)\rangle_{e s}$ e $\tilde{\mathbf{g}}(0)_{e s}$ das eqs.(3.60) e (3.78), respectivamente, obtemos então a densidade espectral $\mathbf{S}(\omega)$ para as variáveis atômicas. Cabe notar que o resultado da eq.(3.87) para a matriz $\Pi$, não permite identificar qual dos termos apresenta uma maior contribuição para a matriz $\mathbf{S}(\omega)$ da eq.(3.86). Na próxima seção tentaremos identificar qual dos termos tem maior contribuição mediante o método perturbativo.

No que diz respeito às densidades espectrais das intensidades dos campos, basta somente substituir a covariância das intensidades dos campos da eq.(3.80) na definição para o espectro de ruído na eq.(1.35), tal que

$$
\begin{aligned}
S_{i j}^{I}(\omega)=\frac{\mu_{i j}}{2 \pi} \int_{-\infty}^{\infty} d \tau e^{-i \omega \tau} & {\left[\left\langle\tilde{\rho}_{i 3}(t+\tau), \tilde{\rho}_{j 3}(t)\right\rangle_{S}-\left\langle\tilde{\rho}_{i 3}(t+\tau), \tilde{\rho}_{3 j}(t)\right\rangle_{S}\right.} \\
& \left.-\left\langle\tilde{\rho}_{3 i}(t+\tau), \tilde{\rho}_{j 3}(t)\right\rangle_{S}+\left\langle\tilde{\rho}_{3 i}(t+\tau), \tilde{\rho}_{3 j}(t)\right\rangle_{S}\right],
\end{aligned}
$$

onde definimos a simetrização como $\langle A, B\rangle_{S}=(\langle A, B\rangle+\langle B, A\rangle) / 2$ para quaisquer variáveis $A$ e $B$. Portanto, a partir das eqs.(3.81)-(3.83), podemos escrever a densidade espectral dos

\footnotetext{
${ }^{13} \mathrm{O}$ movimento Browniano ou difusivo é exemplo de uma evolução estocástica em que seus momentos não convergem.
} 
campos em termos dos elementos da matriz $\mathbf{S}(\omega)$ da eq.(3.86), e assim obtemos

$$
\begin{aligned}
S_{11}^{I}(\omega) & =\mu_{11}\left\{[\mathbf{S}(\omega)]_{33}+[\mathbf{S}(\omega)]_{44}-[\mathbf{S}(\omega)]_{34}-[\mathbf{S}(\omega)]_{43}\right\} \\
S_{22}^{I}(\omega) & =\mu_{22}\left\{[\mathbf{S}(\omega)]_{55}+[\mathbf{S}(\omega)]_{66}-[\mathbf{S}(\omega)]_{56}-[\mathbf{S}(\omega)]_{65}\right\} \\
S_{12}^{I}(\omega) & =\frac{\mu_{12}}{2}\left\{[\mathbf{S}(\omega)]_{36}+[\mathbf{S}(\omega)]_{45}-[\mathbf{S}(\omega)]_{35}-[\mathbf{S}(\omega)]_{46}\right. \\
& \left.+[\mathbf{S}(\omega)]_{63}-[\mathbf{S}(\omega)]_{64}+[\mathbf{S}(\omega)]_{54}-[\mathbf{S}(\omega)]_{53}\right\}
\end{aligned}
$$

onde $S_{11}^{I}(\omega)$ e $S_{22}^{I}(\omega)$ representa a densidade espectral de cada um dos campos, e $S_{12}^{I}(\omega)$ a densidade espectral do produto das intensidades $\left\langle I_{1}(t), I_{2}(t+\tau)\right\rangle_{S}$. Estes espectros representam a densidade espectral dos campos após a interação com os átomos.

Basta então determinar a matriz espectral da eq.(3.86) para determinar os espectros $S_{i j}^{I}(\omega)$ e, assim, finalmente determinar o coeficiente de correlação da eq.(1.36), dado por

$$
C(\omega)=\frac{S_{12}^{I}(\omega)}{\sqrt{S_{11}^{I}(\omega) S_{22}^{I}(\omega)}}
$$

Este coeficiente mede a correlação entre as densidades espectrais de cada um dos campos induzida pelo meio atômico. Em outras palavras, ele mede a correlação entre os ruídos de intensidade dos campos para uma banda lateral com frequência $\omega$, descritas na seção 1.2.3.

\subsubsection{Características da espectroscopia de correlação}

A solução da matriz espectral da eq.(3.86), que permite calcular a correlação da eq.(3.90), tem descrito a espectroscopia de correlação de forma muito próxima aos resultados experimentais reportados em [44]. Nesta seção analisaremos brevemente algumas das suas principais características. Entre elas, o comportamento da correlação com respeito à dessintonia dos campos à ressonância atômica. Isto é chamado: espectroscopia de correlação.

Ao longo da análise que vamos fazer a seguir, consideraremos que ambos os campos possuem as mesmas intensidades, tal que $\Omega_{1}=\Omega_{2}=\Omega$. A figura 3.13 mostra os resultados da espectroscopia de correlação da eq.(3.90) obtidos a partir da solução (3.86) para frequências 
de análise $\omega / 2 \pi=2,3$ e $4 \mathrm{MHz}$, e com dessintonias $\Delta_{2}=0$ e $\Delta_{1}=\delta$. Os espectros da correlação mostram a transição do regime de correlação para anticorrelação à medida que a intensidade aumenta, como foi reportado em [44] e apresentado na figura 3.7.(c). Junto com a correlação mostra-se a absorção dos campos, o que permite identificar a janela de transparência $\Delta \omega_{E I T}$, típica do nosso sistema ${ }^{14}$. A absorção é determinada a partir da solução (3.60), mediante as componentes $\operatorname{Im}\left[\langle\mathbf{x}(t)\rangle_{e s}\right]_{2+i=3,5}=\operatorname{Im} \rho_{i 3}(t)_{e s}$.

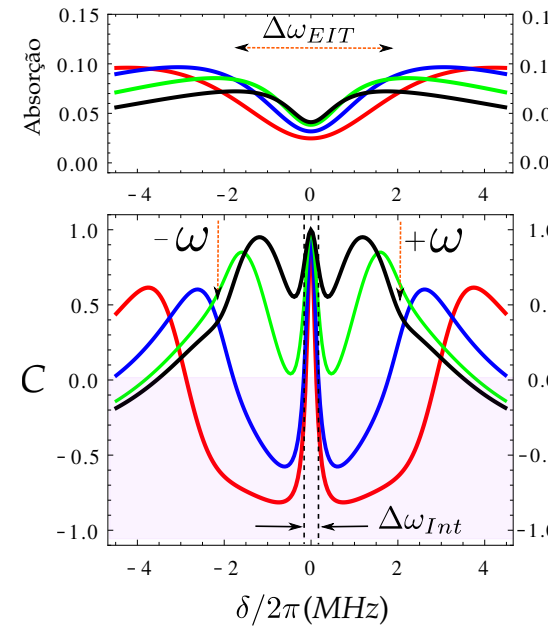

(a)
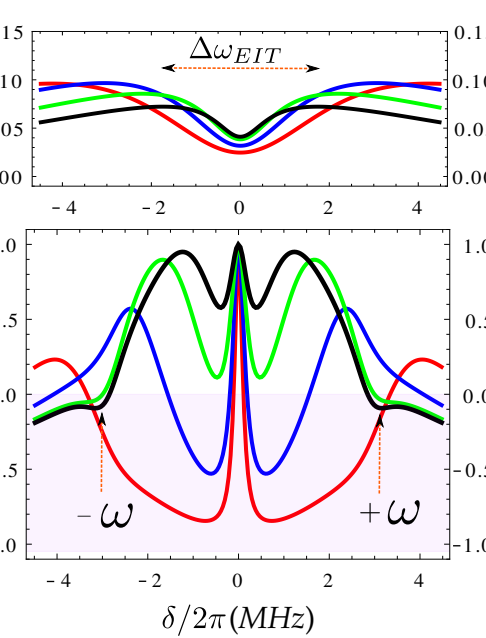

(b)

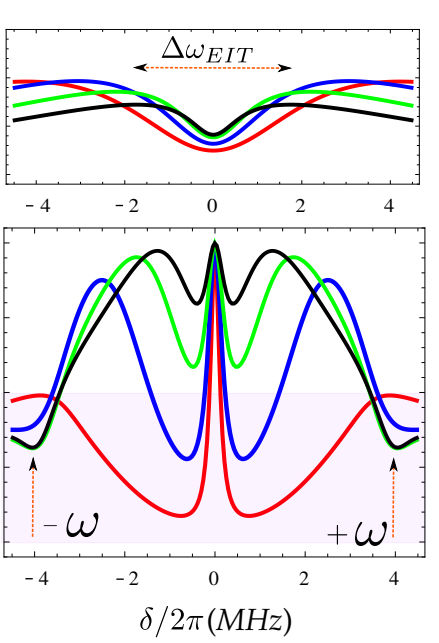

(c)

Figura 3.13: Espectroscopia de absorção e correlação para frequências de análise (a) $\omega / 2 \pi=2 \mathrm{MHz}$, (b) $\omega / 2 \pi=3 \mathrm{MHz}$ e (c) $\omega / 2 \pi=4 \mathrm{MHz}$. Os espectros foram tomados para quatro frequências de Rabi: $\Omega=0.14 \Gamma$ em preto, $\Omega=0.19 \Gamma$ em verde, $\Omega=0.28 \Gamma$ em azul e $\Omega=0.40 \Gamma$ em vermelho. Parâmetros adicionais: $\Gamma / 2 \pi=6.07 \mathrm{MHz}, \bar{\gamma}=1.7 \mathrm{MHz}, \Delta_{2}=0 \mathrm{MHz}$ e $\gamma_{d} / 2 \pi=0.15 \mathrm{kHz}$

Para baixas intensidade foram traçadas as curvas de cor preta e verde, com frequências de Rabi $\Omega=0.14 \Gamma$ e $\Omega=0.19 \Gamma$, respectivamente. Nelas podemos notar que os campos se encontram num regime de correlação $(C>0)$ em torno da ressonância $\delta=0$. Além disso, nelas podemos observar, com certa resolução, um ponto de inflexão na correlação, quando a dessintonia entre as portadoras coincide com a frequência de análise associada às bandas laterais, tal que $\delta=\Delta_{1}-\Delta_{2}=\omega$. Isto se revela claramente quando os pontos de inflexão para $\delta=\omega$ se deslocam ao aumentarmos a frequência de análise $\omega / 2 \pi=2 \mathrm{MHz} \rightarrow \omega / 2 \pi=4$ MHz. Entretanto, as curvas de cor azul e vermelho traçam os espectros de correlação para as

\footnotetext{
${ }^{14} \mathrm{~A}$ janela de transparência $\Delta \omega_{E I T}$ é sensível ao alargamento por potência, ou seja, conforme a intensidade dos campos aumenta, $\Delta \omega_{E I T}$ aumenta.
} 
frequências de Rabi $\Omega=0.28 \Gamma$ e $\Omega=0.40 \Gamma$, respetivamente. Diz-se que o sistema está no regime de anticorrelação porque há valores de dessintonias para as quais $C<0$. A sequência dos quatro espectros revela uma das características mais importantes da espectroscopia de correlação. Diferente da janela de transparência $\Delta \omega_{E I T}$, a largura da ressonância da correlação $\Delta \omega_{\text {Int }}$ (traçadas pelas duas linhas verticais na figura 3.13(a)) se mantêm aproximadamente constantes à medida que aumenta a frequência de Rabi, como acontece com a função $g^{(2)}(0)$ na figura 3.10. Isto mostra que, tanto no domínio do tempo, quanto no domínio das frequências, a largura intrínseca $\Delta \omega_{\text {Int }}$ é livre de alargamento por potência. Como é de se esperar, da mesma maneira que para a função $g^{(2)}(0)$ no domínio da frequência, a largura intrínseca da correlação $C(\omega)$ também está relacionada diretamente com a taxa de decoerência dos níveis fundamentais.

É por esta razão, que dentro do mapa de parâmetros importantes que caracterizam o nosso sistema de três níveis, além da frequência de Rabi e da frequência de análise, temos então a taxa de decoerência $\gamma_{d}$ e a largura de linha dos campos incidentes $\bar{\gamma}$.

Começaremos pela decoerência dos níveis. A figura 3.14.(a) mostra o espectro de correlação para três taxas de decoerência $\gamma_{d}=0.15 \mathrm{MHz}, 0.35 \mathrm{MHz}$ e $0.55 \mathrm{MHz}$, considerando um caso de regime anticorrelacionado com frequência de Rabi é $\Omega=0.24 \Gamma$ e a frequência de análise é $\omega / 2 \pi=2 \mathrm{MHz}$. Notamos que, para as dessintonias assinaladas pelos pontos, o nível de anticorrelação diminui à medida que aumentamos a taxa de decoerência. Por outro lado, a largura intrínseca $\Delta \omega_{\text {Int }}$ apresenta um alargamento proporcional à taxa de decoerência. Isto foi discutido em [43, 44], onde se mostra que a largura intrínseca medida pela espectroscopia de correlação pode ser uma medida robusta da taxa de decoerência entre os níveis fundamentais do sistema $\Lambda$-EIT, pois ela não é sensível ao alargamento por potência.

O último parâmetro a ser analisado é a largura de linha do campo incidente. A figura 3.14.(b) mostra o espectro de correlação para três larguras de linha $\bar{\gamma}=3,14 \mathrm{MHz}, 1,70 \mathrm{MHz}$ e 0,02 MHz. A curva azul mostra o espectro de correlação para $\bar{\gamma}=1,70 \mathrm{MHz}$, que é um valor próximo da largura de banda dos lasers de diodo usados no nosso experimento. A curva vermelha tracejada mostra o espectro de correlação para $\bar{\gamma}=0,02 \mathrm{MHz}$, de modo que quanto menor for o ruído de fase dos campos interagentes, o coeficiente $C$ atinge uma 


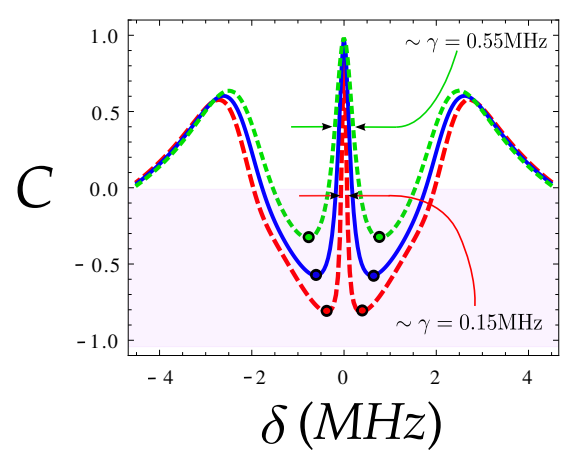

(a)

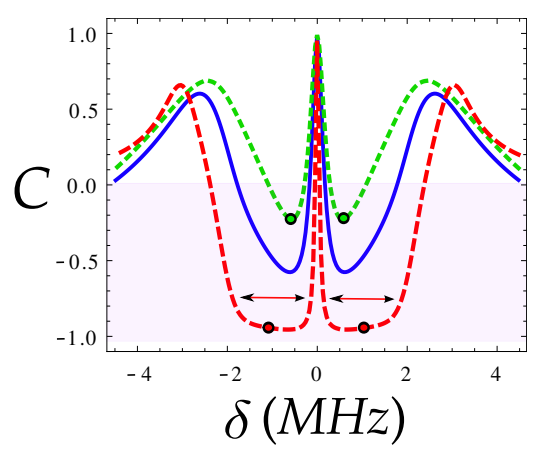

(b)

Figura 3.14: (a) Espectro de correlação para diferentes decoerências: $\gamma_{d}=0,15 \mathrm{kHz}$ traçada em linha vermelha tracejada, $\gamma_{d}=0,35 \mathrm{kHz}$ em linha azul contínua e $\gamma_{d}=0,55 \mathrm{kHz}$ em linha verde tracejada, com larguras de banda do campos $\bar{\gamma}=1.7 \mathrm{MHz}$. (b) Espectro de correlação para larguras espectrais do laser: $\bar{\gamma} / 2 \pi=3.14 \mathrm{MHz}$ traçada em linha verde tracejada, $\bar{\gamma}=1.70 \mathrm{MHz}$ em linha azul contínua $e \bar{\gamma}=0.017 \mathrm{MHz}$ em linha vermelha tracejada, para $\gamma_{d} / 2 \pi=0.15 \mathrm{kHz}$. Parâmetros adicionais: $\Gamma / 2 \pi=6.07 \mathrm{MHz}, \Delta_{2}=0 \mathrm{MHz}, \omega / 2 \pi=2 \mathrm{MHz}, \Omega=0.24 \Gamma$

saturação em valores de anticorrelação dentro da janela de transparência. Ao aumentar a largura de banda para $\bar{\gamma}=3,4 \mathrm{MHz}$ (o espectro é traçado em linha verde tracejada), os átomos induzem menos anticorrelação entre os campos que o caso de $\bar{\gamma}=1,70 \mathrm{MHz}$ (como mostram os pontos de dessintonia verde e vermelho). Contudo, o coeficiente $C$ atinge sempre seu máximo valor na ressonância $\delta=0$, independente da largura de banda dos campos.

Assim, fizemos uma breve análise dos principais parâmetros que caracterizam os espectros de correlação no domínio da frequência. Contudo, a solução obtida a partir do cálculo de Itô se reduz a uma solução numérica. Ela não nos permite extrair uma descrição física de como as variáveis atômicas (absorção e dispersão) induzem uma dada correlação, como é feito com o modelo heurístico da seção 3.4 . 


\subsection{Resultados para a dinâmica átomo-campo mediante o método perturbativo}

Como foi observado na eq.(3.87), a matriz $\Pi$ é o resultado da contribuição dos termos $\langle\mathbf{x}(t)\rangle_{e s}$ e $\tilde{\mathbf{g}}(0)_{e s}$. No entanto, essa solução não permite discriminar de forma analítica qual dos termos contribui em maior medida. Nesta seção, portanto, proponho uma solução para a matriz $\Pi$ mediante uma expansão perturbativa, a qual permite fazer tal distinção. Com ela é possível também estabelecer, finalmente, uma relação direta entre as variáveis de dispersão e absorção com o ruído de intensidade dos campos no domínio das frequências.

\subsubsection{Método perturbativo}

A evolução temporal das variáveis atômicas da eq.(3.56) mostra a relação entre o bombeamento ótico (incluído na matriz $\mathbf{a}[\mathbf{x}, t]$ e proporcional à taxa de decaimento $\Gamma$ ) e a largura de linha do laser (associada à $d \phi$ ), que determina como o sistema é afetado pelo excesso de ruído de fase dos campos incidentes. Podemos então definir a largura de linha do laser como o parâmetro de perturbação $\epsilon=\sqrt{\bar{\gamma}}$. Portanto, redefinimos as flutuações de fase como $d \phi_{i}(t)=\epsilon d W_{i}(t)$, onde $d W_{i}(t)$ é um processo de Wiener que satisfaz $\left\langle d W_{i}(t), d W_{j}(t)\right\rangle=1$. Assim, seguindo o tratamento perturbativo descrito em [52], expandimos o vetor $\mathbf{x}(t)$ como

$$
\mathbf{x}(t)=\mathbf{x}^{(0)}(t)+\epsilon \mathbf{x}^{(1)}(t)+\epsilon^{2} \mathbf{x}^{(2)}(t)+\cdots
$$

Ao substituir esta expansão na eq.(3.60), a solução estacionária do valor médio é reescrito em termos dos elementos de ordem $\mathbf{x}^{(n)}(t)$ :

$$
\begin{aligned}
& \left\langle\mathbf{x}^{(0)}(t)\right\rangle_{s s}=\mathbf{M}^{-1} \mathbf{x}_{o}, \\
& \left\langle\mathbf{x}^{(n)}(t)\right\rangle_{s s}=0 \quad n \geq 1
\end{aligned}
$$


tendo como única contribuição os elementos de ordem $n=0$. Da mesma forma que o vetor $\mathbf{x}$, a matriz de covariâncias $\tilde{\mathbf{g}}(0)$ pode ser expandida em potencias de $\epsilon$, tal que

$$
\tilde{\mathbf{g}}(0)=\epsilon^{2} \sigma^{(2)}+\epsilon^{3} \sigma^{(3)}+\epsilon^{4} \sigma^{(4)}+\epsilon^{5} \sigma^{(5)}+\cdots
$$

onde definimos as covariâncias $\sigma^{(n)}$ como

$$
\begin{aligned}
& \sigma^{(2)}=\left\langle\mathbf{x}^{(1)}(t), \mathbf{x}^{(1)}(t)^{\dagger}\right\rangle, \\
& \sigma^{(3)}=\left\langle\mathbf{x}^{(1)}(t), \mathbf{x}^{(2)}(t)^{\dagger}\right\rangle+\left\langle\mathbf{x}^{(2)}(t), \mathbf{x}^{(1)}(t)^{\dagger}\right\rangle, \\
& \sigma^{(4)}=\left\langle\mathbf{x}^{(2)}(t), \mathbf{x}^{(2)}(t)^{\dagger}\right\rangle+\left\langle\mathbf{x}^{(3)}(t), \mathbf{x}^{(1)}(t)^{\dagger}\right\rangle+\text { c.c, } \\
& \sigma^{(5)}=\left\langle\mathbf{x}^{(2)}(t), \mathbf{x}^{(3)}(t)^{\dagger}\right\rangle+\left\langle\mathbf{x}^{(4)}(t), \mathbf{x}^{(1)}(t)^{\dagger}\right\rangle+\text { c.c, } \\
& \vdots \quad \vdots \quad \vdots \quad .
\end{aligned}
$$

Substituindo estes elementos da expansão na eq.(3.78), obtemos então a fórmula recursiva para obter a solução estacionária das matrizes $\sigma^{(n)}$

$$
\begin{aligned}
\mathbf{M} \sigma_{e s}^{(2 n)}+\sigma_{e s}^{(2 n)} \mathbf{M}^{\dagger} & =2 \Phi \sigma_{e s}^{2(n-1)} \Phi & & n \geq 1 \\
\sigma_{e s}^{(2 n-1)} & =0, & & n \geq 2
\end{aligned}
$$

Todas as matrizes ímpares são nulas, enquanto que as matrizes pares são obtidas recursivamente da matriz de ordem zero, dada pelo produto de vetores

$$
\sigma_{e s}^{(0)}=\left\langle\mathbf{x}_{e s}^{(0)}\right\rangle\left\langle\mathbf{x}_{e s}^{(0)}{ }^{\dagger}\right\rangle
$$

o qual é determinado pela eq. (3.92a).

A partir da expansão da matriz $\tilde{\mathbf{g}}(0)$ e a solução do valor médio $\left\langle\mathbf{x}_{s s}^{(0)}\right\rangle$, a eq. (3.87) agora pode ser escrita como

$$
\Pi=\sum_{n=0}^{\infty} \bar{\gamma}^{n+1} \sigma_{s s}^{(2 n)}
$$


sendo uma expansão explicitamente em potencias da largura de linha do laser $\bar{\gamma}$.

No que diz respeito à matriz de densidade espectral $\mathbf{S}(\omega)$, ao substituir a eq.(3.97) na eq. (3.86), obtemos também a sua expansão em potencias de $\bar{\gamma}$ e, portanto, seus elementos também se expandem como

$$
[\mathbf{S}(\omega)]_{k l}=\sum_{n=0}^{\infty} \bar{\gamma}^{n+1}\left[\mathbf{S}^{(2 n)}(\omega)\right]_{k l}
$$

onde

$$
\mathbf{S}^{(2 n)}(\omega)=\frac{1}{\pi}\left[(\mathbf{M}-i \omega)^{-1} \Phi \sigma_{e s}^{(2 n)} \Phi^{\dagger}\left(\mathbf{M}^{\dagger}+i \omega\right)^{-1}\right] .
$$

Esta solução é um dos resultados mais importantes desta tese. A matriz $\mathbf{S}^{(0)}(\omega)$ representa o principal termo da densidade espectral, qual é determinado pela absorção e dispersão do meio na matriz da eq.(3.96). Termos de ordem superior são considerados para completar a densidade espectral que descreve o sistema.

A expansão perturbativa do espectro de ruído e da correlação cruzada nas eqs.(3.89) são obtidas substituindo os elementos de matriz da eq.(3.98). Portanto, a correlação de intensidade $C(\omega)$ da eq.(3.90) possui diferentes contribuições, dependendo dos termos que forem levados em conta na expansão dos espectros. Temos a mesma situação ao calcular a função de correlação $g^{2}(0)$ (eq. 3.84), devido à expansão perturbativa de $\tilde{\mathbf{g}}(0)$ na eq.(3.93). As figuras 3.15(a) e (b) mostram o resultado obtido pelo método perturbativo para o espectro de correlação $C(\omega)$ e $g^{2}(0)$, respectivamente. Aqui consideramos os mesmos parâmetros empregados nas refs.[43,44] para um regime de anti-correlação. A linha tracejada representa os termos de primeira ordem em $\bar{\gamma}$ associado à matriz $\sigma^{(0)}$. As correções de ordem superior $\bar{\gamma}^{2}$ e $\bar{\gamma}^{3}$ são traçadas em linha pontilhada e linha tracejada-pontilhada, respectivamente. A convergência à solução de Itô é evidente (linha sólida que descreve os parâmetros na ref. [44] e são analisados na seção anterior). Se considerarmos a expansão até termos de ordem $\bar{\gamma}^{2}$, a aproximação seria válida e satisfatória para descrever a correlação de intensidade do sistema.

O limite dos termos relevantes na expansão perturbativa ao calcular a correlação, de- 

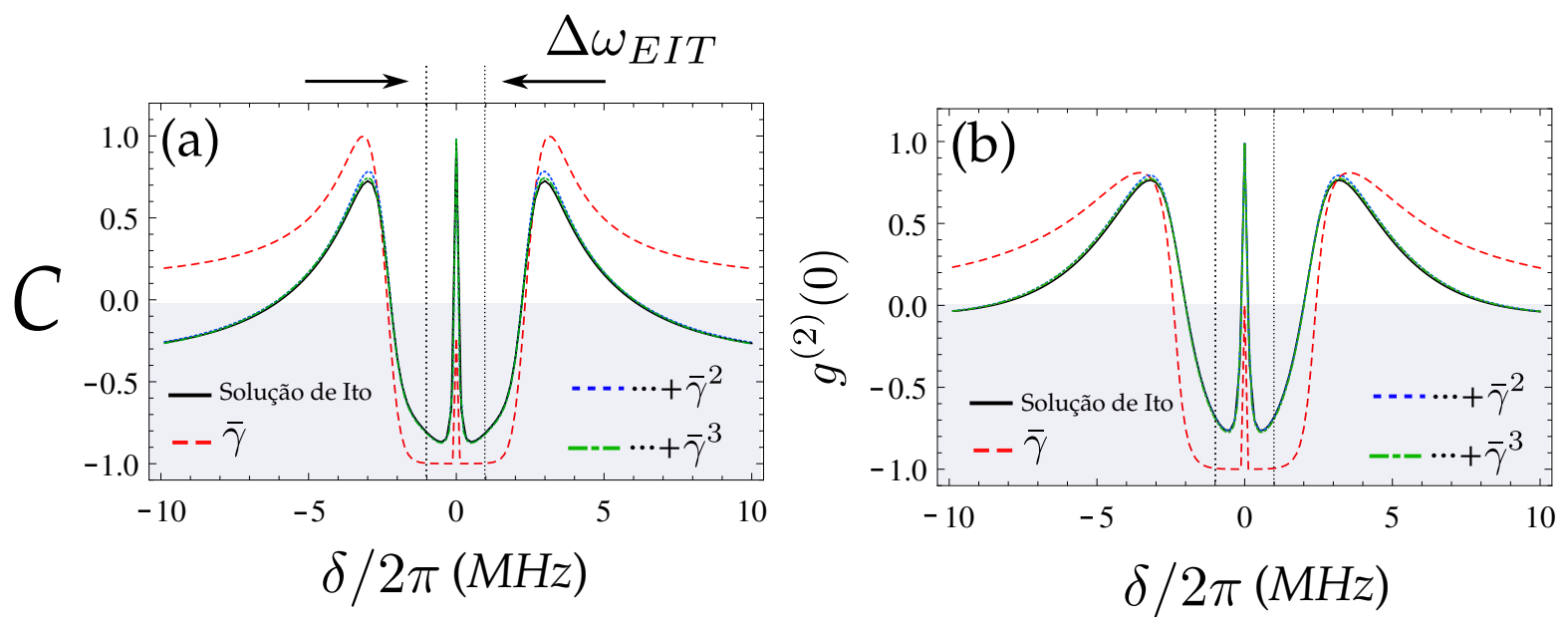

Figura 3.15: Correlação de intensidade em função da dessintonia de dois fótons $\delta=\Delta_{1}-\Delta_{2}$ (ver figura.3.9(a)) entre os dois feixes que interagem com o sistema $\Lambda$ de três níveis. (a) Coeficiente de correlação $C(\omega)$ e (b) função de correlação $g^{2}(0)$. A janela de transparência é $\Delta \omega_{E I T} / 2 \pi \sim 1 M H z$. Parâmetros do cálculo: largura do laser $\bar{\gamma} / 2 \pi=1 \mathrm{MHz}$, frequência de Rabi $\Omega_{1}=\Omega_{2}=0.3 \Gamma$, decoerência dos níveis fundamentais $\gamma_{d} / 2 \pi=150 \mathrm{kHz}$, largura natural $\Gamma / 2 \pi=6 \mathrm{MHz}$, frequências de análise $\omega / 2 \pi=2 \mathrm{MHz}$ e dessintonia de um fóton $\Delta_{2}=0$.

pende evidentemente da largura de linha do laser, como mostram as figuras 3.16(a) e (b). Nelas comparamos os espectros de correlação para larguras de linha da ordem de $\bar{\gamma} / 2 \pi=10 \mathrm{MHz}$ (excesso de ruído acima da média ${ }^{15}$ ) e $\bar{\gamma} / 2 \pi=0.01 \mathrm{MHz}$ (excesso de ruído abaixo da média). Para o caso acima da média, a figura 3.16(a) mostra que é necessários termos de ordem superior a $\bar{\gamma}^{3}$ para determinar por completo o espectro de correlação. Por outro lado, a figura 3.16(b) mostra que, para largura abaixo da média, o espectro de correlação é completamente descrito pelo termo de primeira ordem $\bar{\gamma}$, o qual reproduz a solução obtida por Itô na eq.(3.90). Isto demonstra que para fontes de luz coerentes (que não apresentam excesso de ruído de fase), a aproximação linear padrão dos observáveis físicos, neste caso as variáveis atômicas $\mathbf{x}(t)=\langle\mathbf{x}(t)\rangle+\delta \mathbf{x}(t)$, é valida e suficiente.

Esta expansão perturbativa nos motivou à busca de uma expressão explícita para $C(\omega)$, análogo ao que foi feito com a função de correlação $g^{2}(0)$ mediante o modelo heurístico (seção 3.4). O meu objetivo é encontrar uma expressão que relacione a absorção e a dispersão dos campos com a correlação no domínio da frequência.

\footnotetext{
${ }^{15}$ Consideramos a largura média como a largura típica dos nossos lasers de diodo, $\bar{\gamma} / 2 \pi=1 \mathrm{MHz}$.
} 

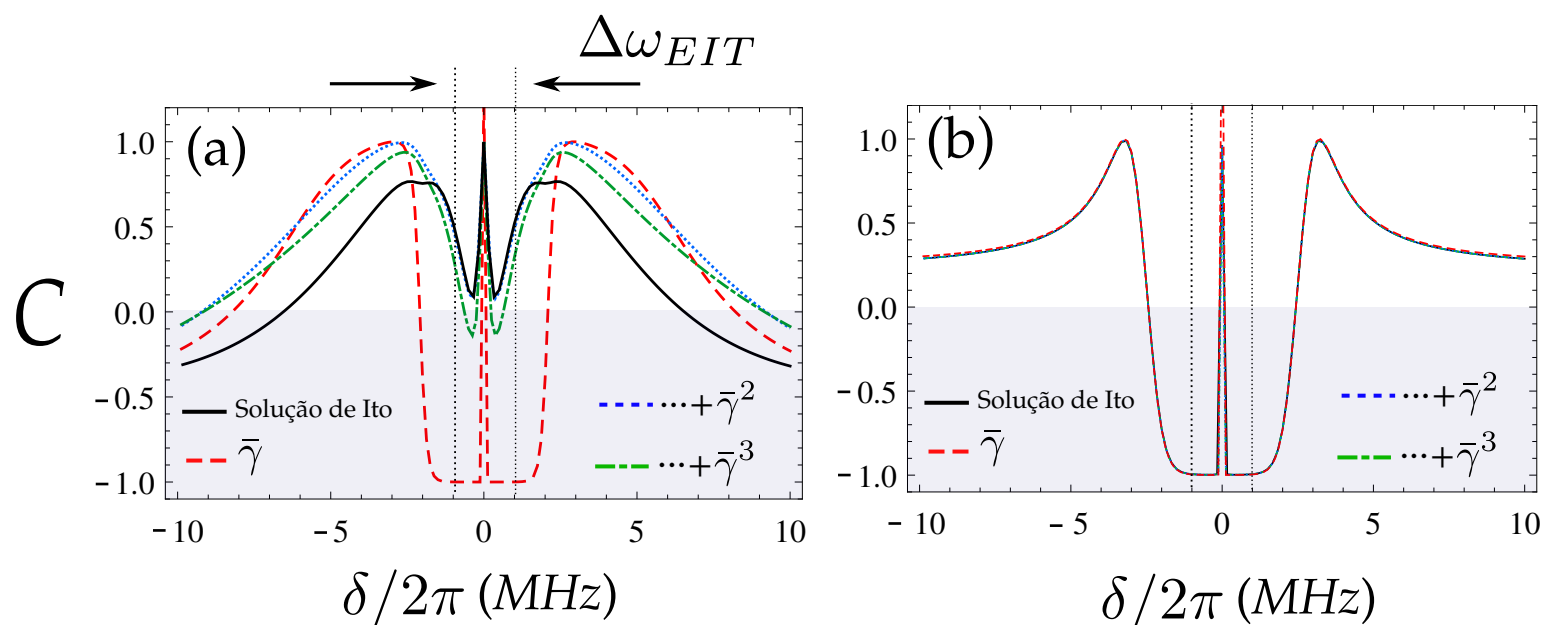

Figura 3.16: Espectros de correlação para larguras de linha do laser: (a) $\bar{\gamma} / 2 \pi=10 \mathrm{MHz}$ e (b) $\bar{\gamma} / 2 \pi=0.01 \mathrm{MHz}$. Os parâmetros do cálculo são os mesmos da figura 3.15. As duas linhas verticais pontilhadas representam a janela de transparência $\Delta \omega_{E I T}$.

\subsubsection{Mapeamento da absorção e dispersão com o espectro de correlação}

A expansão perturbativa da matriz П na eq.(3.97), mostra como o termo de primeira ordem, o qual depende diretamente da absorção e dispersão dos campos, estabelece o principal termo para a matriz de densidade espectral. A partir deste primeiro termo, podemos determinar correções de ordem superior. É devido a essa relação entre parte real e parte imaginária com o primeiro termo da matriz $\Pi$, que achei necessário aplicar uma transformação que inclua todos os termos da expansão, deixando-a toda ela explícita em termos de parte real e imaginária dos seu elementos. Isto traz uma consequência imediata: a decomposição da matriz $\mathbf{S}(\omega)$ em termos da absorção e dispersão, análogo ao que foi feito no modelo heurístico da eq.(3.35).

Começaremos descrevendo a transformação da matriz П. O seu primeiro termo, a matriz $\sigma^{(0)}$ definida na eq.(3.96), é calculada a partir do termo de ordem zero $\left\langle\mathbf{x}_{s t}^{(0)}\right\rangle=\left(\rho_{11}^{(0)}, \rho_{22}^{(0)}, p_{1}, p_{1}^{*}\right.$, $\left.p_{2}, p_{2}^{*}, \rho_{12}^{(0)}, \rho_{21}^{(0)}\right)$, cuja solução estacionaria é dada na eq.(3.92a). Outra forma de descrever o sistema é mediante o vetor $\tilde{\mathbf{x}}=\left(\rho_{11}^{(0)}, \rho_{22}^{(0)}, \operatorname{Re} p_{1}, \operatorname{Im} p_{1}, \operatorname{Re} p_{2}, \operatorname{Im} p_{2}, \rho_{12}^{(0)}, \rho_{21}^{(0)}\right)$, que é explicitamente dependente da absorção e da dispersão dos campos. Portanto, podemos aplicar a 
transformação unitária $\mathbf{U}$ que relaciona os dois vetores $\left\langle\mathbf{x}_{\mathbf{s t}}^{(\mathbf{0})}\right\rangle$ e $\tilde{\mathbf{x}}$, tal que

$$
\tilde{\mathbf{x}}=\mathbf{U}\left\langle\mathbf{x}_{s t}^{0}\right\rangle=\left[\begin{array}{cccccccc}
1 & 0 & 0 & 0 & 0 & 0 & 0 & 0 \\
0 & 1 & 0 & 0 & 0 & 0 & 0 & 0 \\
0 & 0 & \frac{1}{2} & \frac{1}{2} & 0 & 0 & 0 & 0 \\
0 & 0 & -\frac{i}{2} & \frac{i}{2} & 0 & 0 & 0 & 0 \\
0 & 0 & 0 & 0 & \frac{1}{2} & \frac{1}{2} & 0 & 0 \\
0 & 0 & 0 & 0 & -\frac{i}{2} & \frac{i}{2} & 0 & 0 \\
0 & 0 & 0 & 0 & 0 & 0 & 1 & 0 \\
0 & 0 & 0 & 0 & 0 & 0 & 0 & 1
\end{array}\right]\left[\begin{array}{c}
\rho_{11}^{(0)} \\
\rho_{22}^{(0)} \\
p_{1} \\
p_{1}^{*} \\
p_{2} \\
p_{2}^{*} \\
\rho_{12}^{(0)} \\
\rho_{21}^{(0)}
\end{array}\right]=\left[\begin{array}{c}
\rho_{11}^{(0)} \\
\rho_{22}^{(0)} \\
\operatorname{Re} p_{1} \\
\operatorname{Im} p_{1} \\
\operatorname{Re} p_{2} \\
\operatorname{Im} p_{2} \\
\rho_{12}^{(0)} \\
\rho_{21}^{(0)}
\end{array}\right]
$$

Em seguida, aplicamos a transformação (3.100) na matriz de densidade espectral (3.99), tal que

$$
\begin{aligned}
\mathbf{S}(\omega) & =\frac{1}{2 \pi}(\mathbf{M}-i \omega)^{-1} \mathbf{U}^{-1} \mathbf{U} \Phi \Pi \Phi \mathbf{U}^{-1} \mathbf{U}\left(\mathbf{M}^{\dagger}+i \omega\right)^{-1} \\
& =\frac{1}{2 \pi}\left[\Lambda_{L}(-\omega) \tilde{\Pi} \Lambda_{R}(\omega)\right]
\end{aligned}
$$

onde definimos

$$
\begin{aligned}
\tilde{\Pi} & =\mathbf{U} \Phi \Pi \Phi \mathbf{U}^{-1} \\
\Lambda_{L}(-\omega) & =(\mathbf{M}-i \omega)^{-1} \mathbf{U}^{-1} \\
\Lambda_{R}(\omega) & =\mathbf{U}\left(\mathbf{M}^{\dagger}+i \omega\right)^{-1} .
\end{aligned}
$$

De acordo com a definição da eq.(3.87), a nova matriz П̃ pode ser escrita como

$$
\tilde{\Pi}=\sum_{n=0}^{\infty} \bar{\gamma}^{n+1} \tilde{\sigma}^{(2 n)}
$$

onde $\tilde{\sigma}^{(2 n)}=\mathbf{U} \Phi \sigma^{(2 n)} \Phi \mathbf{U}^{-1}$.

A representação matricial de $\tilde{\sigma}^{(2 n)}$ depende do produto $\Phi \sigma_{e s}^{(2 n)} \Phi$, que é escrito matricial- 
mente como

$$
\Phi \sigma_{e s}^{(2 n)} \Phi=\left[\begin{array}{cccccccc}
0 & 0 & 0 & 0 & 0 & 0 & 0 & 0 \\
0 & 0 & 0 & 0 & 0 & 0 & 0 & 0 \\
0 & 0 & \sigma_{33}^{(2 n)} & -\sigma_{34}^{(2 n)} & \sigma_{35}^{(2 n)} & -\sigma_{36}^{(2 n)} & 0 & 0 \\
0 & 0 & -\sigma_{43}^{(2 n)} & \sigma_{44}^{(2 n)} & -\sigma_{45}^{(2 n)} & \sigma_{46}^{(2 n)} & 0 & 0 \\
0 & 0 & \sigma_{53}^{(2 n)} & -\sigma_{54}^{(2 n)} & \sigma_{55}^{(2 n)} & -\sigma_{56}^{(2 n)} & 0 & 0 \\
0 & 0 & -\sigma_{63}^{(2 n)} & \sigma_{64}^{(2 n)} & -\sigma_{65}^{(2 n)} & \sigma_{66}^{(2 n)} & 0 & 0 \\
0 & 0 & 0 & 0 & 0 & 0 & 0 & 0 \\
0 & 0 & 0 & 0 & 0 & 0 & 0 & 0
\end{array}\right]
$$

o que reduz o número de elementos independentes de $8 \times 8=64$ (da matriz $\sigma_{e s}^{(2 n)}$ ) para $4 \times 4=16\left(\right.$ da matriz $\left.\Phi \sigma_{e s}^{(2 n)} \Phi\right)$. Portanto, a nova matriz de ordem zero $\tilde{\sigma}_{i j}^{(0)}$, por exemplo, é representada por

$$
\tilde{\sigma}^{(0)}=2\left[\begin{array}{cccccccc}
0 & 0 & 0 & 0 & 0 & 0 & 0 & 0 \\
0 & 0 & 0 & 0 & 0 & 0 & 0 & 0 \\
0 & 0 & \operatorname{Im}^{2} p_{1} & -\operatorname{Im} p_{1} \operatorname{Re} p_{1} & \operatorname{Im} p_{1} \operatorname{Im} p_{2} & -\operatorname{Im} p_{1} \operatorname{Re} p_{2} & 0 & 0 \\
0 & 0 & -\operatorname{Im} p_{1} \operatorname{Re} p_{1} & \operatorname{Re}^{2} p_{1} & -\operatorname{Im} p_{2} \operatorname{Re} p_{1} & \operatorname{Re} p_{1} \operatorname{Re} p_{2} & 0 & 0 \\
0 & 0 & \operatorname{Im} p_{1} \operatorname{Im} p_{2} & -\operatorname{Im} p_{2} \operatorname{Re} p_{1} & \operatorname{Im}^{2} p_{2} & -\operatorname{Im} p_{2} \operatorname{Re} p_{2} & 0 & 0 \\
0 & 0 & -\operatorname{Im} p_{1} \operatorname{Re} p_{2} & \operatorname{Re} p_{1} \operatorname{Re} p_{2} & -\operatorname{Im} p_{2} \operatorname{Re} p_{2} & \operatorname{Re}^{2} p_{2} & 0 & 0 \\
0 & 0 & 0 & 0 & 0 & 0 & 0 & 0 \\
0 & 0 & 0 & 0 & 0 & 0 & 0 & 0
\end{array}\right] .
$$

As matrizes de ordem superior seguem a transformação da eq.(3.105), mantendo a mesma forma da matriz de ordem zero (3.107). Portanto, os elementos de matriz $\tilde{\Pi}_{i j}$ são descritos 
pelo conjunto de elementos associados às coerências entre os níveis 1 e 3

$$
\begin{aligned}
& \tilde{\Pi}_{33}=\bar{\gamma}\left(2 \operatorname{Im}^{2} p_{1}+\sum_{n=1}^{\infty} \bar{\gamma}^{n} \tilde{\sigma}_{33}^{(2 n)}\right), \\
& \tilde{\Pi}_{44}=\bar{\gamma}\left(2 \operatorname{Re}^{2} p_{1}+\sum_{n=1}^{\infty} \bar{\gamma}^{n} \tilde{\sigma}_{44}^{(2 n)}\right), \\
& \tilde{\Pi}_{34}=\bar{\gamma}\left(-2 \operatorname{Im} p_{1} \operatorname{Re} p_{1}+\sum_{n=1}^{\infty} \bar{\gamma}^{n} \tilde{\sigma}_{34}^{(2 n)}\right),
\end{aligned}
$$

um outro conjunto associado às coerências entre os níveis 2 e 3

$$
\begin{aligned}
& \tilde{\Pi}_{55}=\bar{\gamma}\left(2 \operatorname{Im}^{2} p_{2}+\sum_{n=1}^{\infty} \bar{\gamma}^{n} \tilde{\sigma}_{55}^{(2 n)}\right), \\
& \tilde{\Pi}_{66}=\bar{\gamma}\left(2 \operatorname{Re}^{2} p_{2}+\sum_{n=1}^{\infty} \bar{\gamma}^{n} \tilde{\sigma}_{66}^{(2 n)}\right), \\
& \tilde{\Pi}_{56}=\bar{\gamma}\left(-2 \operatorname{Im} p_{2} \operatorname{Re} p_{2}+\sum_{n=1}^{\infty} \bar{\gamma}^{n} \tilde{\sigma}_{56}^{(2 n)}\right),
\end{aligned}
$$

e um conjunto final que envolve termos cruzados de ambas as coerências

$$
\begin{aligned}
& \tilde{\Pi}_{35}=\bar{\gamma}\left(2 \operatorname{Im} p_{1} \operatorname{Im} p_{2}+\sum_{n=1}^{\infty} \bar{\gamma}^{n} \tilde{\sigma}_{35}^{(2 n)}\right), \\
& \tilde{\Pi}_{46}=\bar{\gamma}\left(2 \operatorname{Re} p_{1} \operatorname{Re} p_{2}+\sum_{n=1}^{\infty} \bar{\gamma}^{n} \tilde{\sigma}_{46}^{(2 n)}\right), \\
& \tilde{\Pi}_{36}=\bar{\gamma}\left(-2 \operatorname{Im} p_{1} \operatorname{Re} p_{2}+\sum_{n=1}^{\infty} \bar{\gamma}^{n} \tilde{\sigma}_{36}^{(2 n)}\right), \\
& \tilde{\Pi}_{45}=\bar{\gamma}\left(-2 \operatorname{Im} p_{2} \operatorname{Re} p_{1}+\sum_{n=1}^{\infty} \bar{\gamma}^{n} \tilde{\sigma}_{45}^{(2 n)}\right) .
\end{aligned}
$$

Assim, a partir da transformação (3.101), podemos então expandir os elemento da matriz de 
densidade espectral $[\mathbf{S}(\omega)]_{i j}$ na base dos elementos $\tilde{\Pi}_{i j}$, como

$$
[\mathbf{S}(\omega)]_{k l}=\sum_{i, j=3}^{6} V_{k i j l} \tilde{\Pi}_{i j}
$$

onde $V_{k i j l}=\left[\Lambda_{L}(\omega)\right]_{k i}\left[\Lambda_{R}(-\omega)\right]_{j l}$. A soma exclui os elementos com índice $i=1,2,7$ e 8 , pois

$$
\begin{array}{rlrl}
\tilde{\Pi}_{1 i} & =\tilde{\Pi}_{i 1}=0, & \tilde{\Pi}_{2 i}=\tilde{\Pi}_{i 2}=0, \\
\tilde{\Pi}_{7 i}=\tilde{\Pi}_{i 7}=0, & \tilde{\Pi}_{8 i}=\tilde{\Pi}_{i 8}=0,
\end{array}
$$

devido aos elementos nulos da matriz (3.106).

No que diz respeito aos espectros de ruído dos campos, são determinados a partir dos elementos de matriz da eq.(3.111). Enquanto a matriz (3.107) contém todos os elementos necessários para calcular a função $g^{(2)}(0)$ do modelo heurístico da eq.(3.35), no domínio da frequência, os espectros de ruído das eqs.(3.89a)-(3.89c) são dados pelo produto da expansão das covariâncias $\tilde{\Pi}_{i j}$, modulados por fatores que dependem da frequência de analise $\omega$ :

$$
\begin{aligned}
& S^{I}(\omega)_{11}=\sum_{i, j=3}^{6} \alpha_{i j}(\omega) \tilde{\Pi}_{i j}, \\
& S^{I}(\omega)_{22}=\sum_{i, j=3}^{6} \beta_{i j}(\omega) \tilde{\Pi}_{i j} \\
& S^{I}(\omega)_{12}=\sum_{i, j=3}^{6} \nu_{i j}(\omega) \tilde{\Pi}_{i j}
\end{aligned}
$$

onde

$$
\begin{aligned}
\alpha_{i j}(\omega) & =\left[V_{3 i j 3}(\omega)+V_{4 i j 4}(\omega)-V_{3 i j 4}(\omega)-V_{4 i j 3}(\omega)\right], \\
\beta_{i j}(\omega) & =\left[V_{5 i j 5}(\omega)+V_{6 i j 6}(\omega)-V_{5 i j 6}(\omega)-V_{6 i j 5}(\omega)\right],
\end{aligned}
$$




$$
\begin{aligned}
\nu_{i j}(\omega)= & 1 / 2\left[V_{3 i j 6}(\omega)+V_{4 i j 5}(\omega)-V_{3 i j 5}(\omega)-V_{4 i j 6}(\omega)\right. \\
& \left.+V_{6 i j 3}(\omega)-V_{6 i j 4}(\omega)+V_{5 i j 4}(\omega)-V_{5 i j 3}(\omega)\right] .
\end{aligned}
$$

Se mantivermos a expansão dos elementos $\tilde{\Pi}_{i j}$ até primeira ordem, de acordo com as eqs.(3.114)(3.116), o espectro de correlação é escrito como

$$
C(\omega)=\frac{\tilde{\nu}_{35}(\omega) \operatorname{Im} p_{1} \operatorname{Im} p_{2}+\tilde{\nu}_{46}(\omega) \operatorname{Re} p_{1} \operatorname{Re} p_{2}+\tilde{\nu}_{36}(\omega) \operatorname{Im} p_{1} \operatorname{Re} p_{2}+\tilde{\nu}_{45}(\omega) \operatorname{Im} p_{2} \operatorname{Re} p_{1}+C_{1 \mid 2}(\omega)}{\sqrt{\left[\alpha_{33}(\omega) \operatorname{Im}^{2} p_{1}+\alpha_{44}(\omega) \operatorname{Re}^{2} p_{1}+C_{11}(\omega)\right]\left[\beta_{55}(\omega) \operatorname{Im}^{2} p_{2}+\beta_{66}(\omega) \operatorname{Re}^{2} p_{2}+C_{22}(\omega)\right]}}
$$

onde $C_{11}(\omega), C_{22}(\omega)$ e $C_{1 \mid 2}(\omega)$ são definidos no apêndice $\mathrm{A}$ nas eqs. (A.2)-(A.6). Este resultado é análogo ao da função de correlação do modelo heurístico na eq.(3.35). No entanto, em vez de ter $\operatorname{Im} p_{1} \operatorname{Im} p_{2}$ e $\operatorname{Re} p_{2} \operatorname{Re} p_{1}$ com contribuições iguais na correlação, os fatores $\nu_{35}(\omega)$ e $\nu_{46}(\omega)$ modulam a sua contribuição na eq.(3.120).

Por outro lado, além da contribuição dos termos $\operatorname{Im} p_{1} \operatorname{Re} p_{2}$ e $\operatorname{Im} p_{2} \operatorname{Re} p_{1}$, todos os possíveis termos cruzados da eq. (3.107) também contribuem para os espectros $S_{12}^{I}$, $S_{11}^{I}$ e $S_{22}^{I}$. Mais adiante veremos que os termos $C_{11}, C_{22}$ e $C_{12}$ são relevantes, porém, sua contribuição é menor à dos termos explicitamente descritos.

Chegamos então ao objetivo desta seção: decompor a correlação de tal forma que, pela sua nova expressão analítica, podemos analisar cada um dos seus elementos separadamente, aqueles dependentes e os independentes da frequência $\omega$. Ao combiná-los mediante a solução (3.120), determinamos um certo nível de correlação no domínio da frequência.

Desse elementos começaremos analisando os elementos independentes da frequência $\omega$, ou seja, os elementos $\tilde{\Pi}_{i j}$. Na figura 3.17 podemos observar o efeito das correções perturbativas nos elementos $\tilde{\Pi} / \bar{\gamma}$, considerando valores típicos $\bar{\gamma} / 2 \pi=1 \mathrm{MHz}$ e $\Delta_{2}=0$. As figuras (a), (b) e (c) mostram o efeito das correções perturbativas em torno de $\operatorname{Im}^{2} p_{i}, \operatorname{Re}^{2} p_{i}$ e $\operatorname{Im} p_{i} \operatorname{Re} p_{i}$, respectivamente, para os dois campos $i=1$, 2. Entretanto, as figuras (c) e (d) mostram as correções perturbativas para $\operatorname{Im} p_{1} \operatorname{Im} p_{2}\left(\operatorname{Re} p_{1} \operatorname{Re} p_{2}\right)$ e para os produtos $\operatorname{Im} p_{1} \operatorname{Re} p_{2}\left(\operatorname{Re} p_{1} \operatorname{Im} p_{2}\right)$. Podemos observar que o termo de ordem menor segue de perto o perfil que leva em conta a 
expansão completa. $\tilde{\Pi}_{i j} / \bar{\gamma}$.

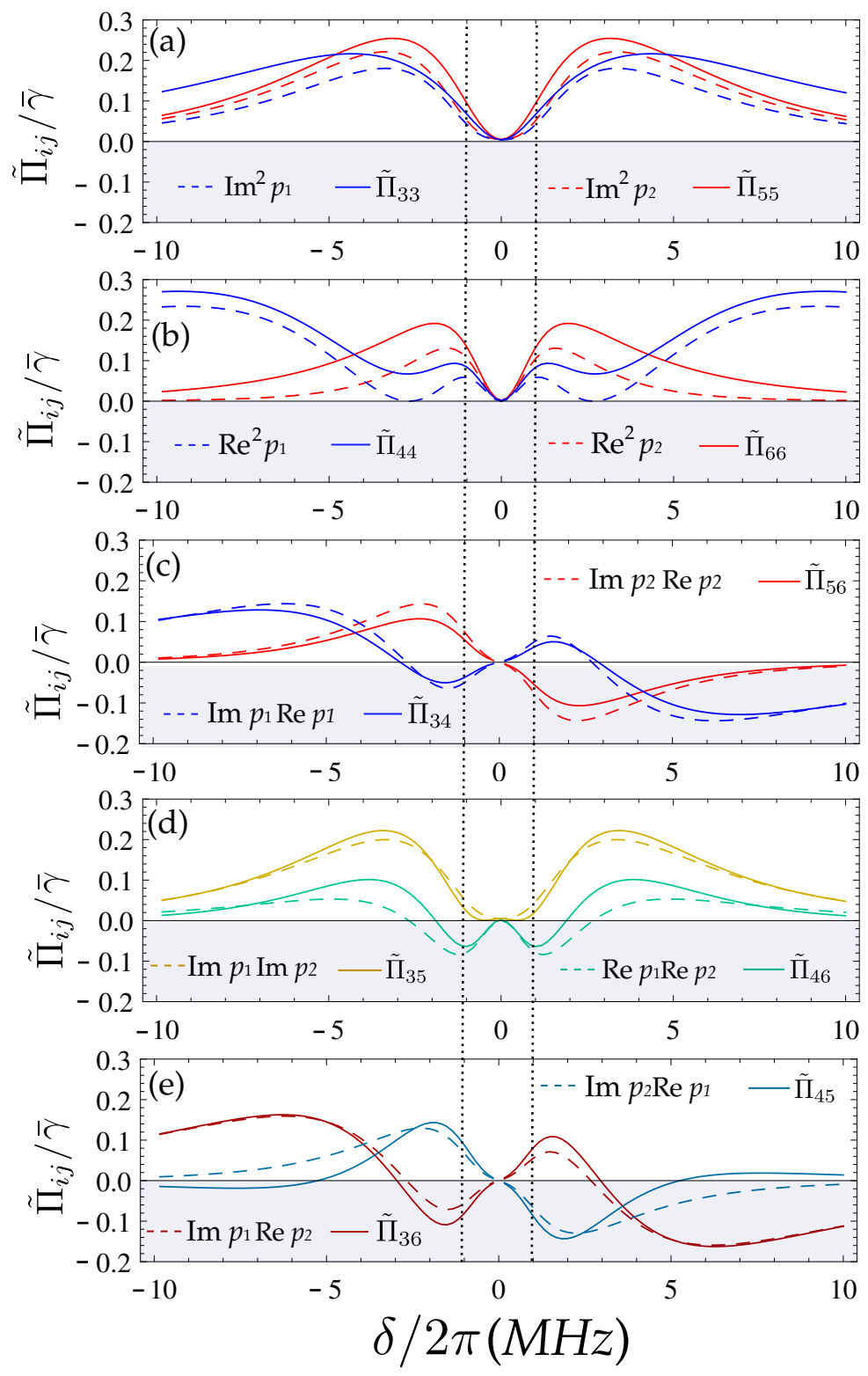

Figura 3.17: Comparação dos elementos de matriz à primeira ordem $\left(\sigma_{i j}^{(0)}\right)$ com os termos de ordem superior na expansão perturbativa $\tilde{\Pi}_{i j} / \bar{\gamma}=\sigma_{i j}^{(0)}+\cdots+\bar{\gamma}^{6} \sigma_{i j}^{(7)}$. (a) Para a resposta de absorção e (b) a de dispersão para cada campo. (c) Para produto da absorção $\times$ dispersão para cada campo. (d) e (e) para os produtos cruzados $\operatorname{Im} p_{1} \operatorname{Im} p_{2}\left(\operatorname{Rep}_{1} \operatorname{Rep}_{2}\right)$ e Imp $\operatorname{Iep}_{1} \operatorname{Re}_{2}\left(\operatorname{Rep}_{1} \operatorname{Im} p_{2}\right)$. Os parâmetros para obter os espectros são os mesmos considerados na figura 3.15. As duas linhas verticais representam a janela de transparência $\Delta \omega_{E I T}$.

Agora, ao comparar a resposta dispersiva com a resposta de absorção, as figuras 3.17(a) 
e (b), mostram que $\operatorname{Im}^{2} p_{i} \sim \operatorname{Re}^{2} p_{i}$ para $|\delta|<\Delta \omega_{E I T}$. No entanto, para $|\delta|>\Delta \omega_{E I T}$, a resposta atômica apresenta situações em que a absorção ultrapassa a contribuição da dispersão, ou vice versa. O produto $\operatorname{Im} p_{i} \operatorname{Re} p_{i}$ também tem um papel importante na resposta atômica de cada campo, tendo um perfil muito próximo ao da figura (c). Notemos que os coeficientes $\alpha_{i j}(\omega), \beta_{i j}(\omega)$ e $\nu_{i j}(\omega)$ serão os que determinam qual dos termos se tornam a principal contribuição em cada intervalo de frequências do espectro que analisarmos.

Por outro lado, o produto cruzado $\operatorname{Re} p_{1} \operatorname{Re} p_{2}$ compete com o termo $\operatorname{Im} p_{1} \operatorname{Im} p_{2}$ para dessintonias $|\delta|<\Delta \omega_{E I T}$, como mostra a figura 3.17(d). Somente os coeficientes $\nu_{i j}(\omega)$ vão determinar qual termo contribui em maior medida para induzir correlação ou anti-correlação. No caso em que $|\delta| / 2 \pi>6 \mathrm{MHz}$, o termo de absorção $\left(\tilde{\Pi}_{35}\right)$ e de dispersão $\left(\tilde{\Pi}_{46}\right)$ são positivos e contribuem para $C(\omega)>0$.

Finalmente, a figura 3.17(e) mostra os produtos cruzados $\operatorname{Im} p_{i} \operatorname{Re} p_{j}$, que apresentam um perfil do tipo dispersivo. Estes termos não aparecem na solução de $g^{2}(0)$ do modelo heurístico. Entre as várias características relevantes, cabe notar que $\tilde{\Pi}_{45}$ é o único elemento de matriz que inverte seu sinal para dessintonias $|\delta| / 2 \pi>6 \mathrm{MHz}$, quando consideramos os termos de ordem superior. Este elemento de matriz é então o responsável pela inversão do sinal no espectro de correlação da figura 3.15(a) para o mesmo intervalo de dessintonias, quando levamos em conta termos de ordem superior no seu cálculo.

Agora vamos analisar os elementos dependentes da frequência de análise $\omega$. Os coeficientes $\alpha_{i j}(\omega), \beta_{i j}(\omega)$ e $\nu_{i j}(\omega)$ vão determinar a contribuição efetiva dos elementos de matriz $\tilde{\Pi}_{i j}$ para os espectros de ruído e de correlação de intensidade dos campos. A figura 3.18(a) mostra os espectros de ruído $S_{11}(\omega)$ e $S_{22}(\omega)$, calculado com termos até ordem $\bar{\gamma}^{7}$ para determinar П̃. Entretanto, as figuras 3.18(b) e (c) mostram a contribuição dos produtos $\alpha_{i j} \Pi_{i j}$ associados a $S_{11}$. Da mesma forma, as figuras 3.18(d) e (e) mostram os produtos $\beta_{i j} \Pi_{i j}$ associados a $S_{22}$.

Ao contrário do modelo heurístico para $g^{2}(0)$, em que $\operatorname{Im}^{2} p_{i}$ e $\operatorname{Re}^{2} p_{i}$ contribuem na mesma proporção aos ruídos dos campos, as figuras 3.18(b) e (e) mostram que, dentro do intervalo $|\delta|<\Delta \omega_{E I T}$, a reposta dispersiva $\left(\tilde{\Pi}_{44}\right.$ e $\left.\tilde{\Pi}_{66}\right)$ ultrapassam a resposta pela absorção $\left(\tilde{\Pi}_{33}\right.$ e $\left.\tilde{\Pi}_{55}\right)$, dado que $\alpha_{44} \tilde{\Pi}_{44}>\alpha_{33} \tilde{\Pi}_{33}$ e $\beta_{66} \tilde{\Pi}_{66}>\beta_{55} \tilde{\Pi}_{55}$, respectivamente. Em particu- 

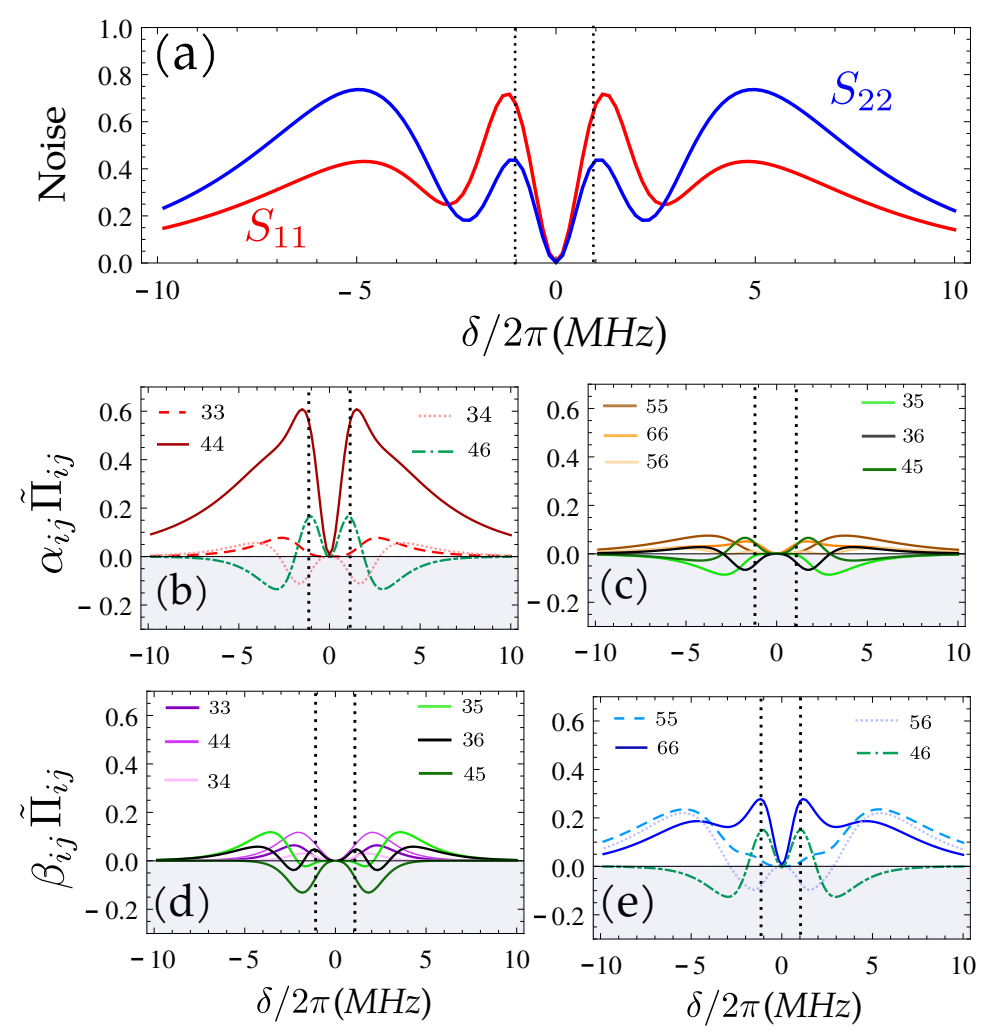

Figura 3.18: Decomposição dos espectros de ruído $S_{11}$ e $S_{22}$ em termos dos coeficientes $\alpha_{i j} \beta_{i j}$, respectivamente. Os espectros de ruído em (a) são calculados até ordem $\bar{\gamma}^{7}$ com uma frequência de análise $\omega / 2 \pi=2 \mathrm{MHz}$. (b) e (e) mostram os principais coeficientes $\alpha_{i j}$ e $\beta_{i j}$, respectivamente. (c) Mostra os coeficientes de menor contribuição $\alpha_{55}, \alpha_{66}, \alpha_{56}, \alpha_{35}, \alpha_{36}$ e $\alpha_{45}(d)$ Mostra os coeficientes de menor contribuição $\beta_{33}, \beta_{44}, \beta_{34}, \beta_{35}, \beta_{36}$ e $\beta_{45}$. Os parâmetros para obter os espectros são os mesmos considerados na figura 3.15. As duas linhas verticais representam a janela de transparência $\Delta \omega_{E I T}$.

lar, para $|\delta| \sim \Delta \omega_{E I T}$, as componentes $\alpha_{44} \tilde{\Pi}_{44}$ e $\beta_{66} \tilde{\Pi}_{66}$ se tornam a contribuição principal para os espectros $S_{11}(\omega)$ e $S_{22}(\omega)$ da figura 3.18.(a), respectivamente. No entanto, podemos observar que os outros termos são importantes para $|\delta| \geq \Delta \omega_{E I T}$, como por exemplo, as contribuição de $\alpha_{46}\left(\beta_{46}\right)$, associada a $\operatorname{Re} p_{1} \operatorname{Re} p_{2}$ (figura 3.17c). Além disso, para esse intervalo de frequências, o termo associado à absorção $\beta_{55} \tilde{\Pi}_{55}$ e o termo associado à dispersão $\beta_{66} \tilde{\Pi}_{66}$ apresentam quase a mesma contribuição para o espectro $S_{22}(\omega)$, diferente de $S_{11}(\omega)$, em que a dispersão associada a $\alpha_{44} \tilde{\Pi}_{44}$ ainda ultrapassa a dispersão associada a $\alpha_{33} \tilde{\Pi}_{33}$. Outros termos importantes são os modulados por $\alpha_{34}$ e $\beta_{56}$ (associados a $\operatorname{Im} p_{i} \operatorname{Re} p_{i}$ tendo $i=1,2$ ), que também apresentam um efeito notório. As figuras 3.18(c) e (d) mostram os coeficientes restantes $\alpha_{i j} \tilde{\Pi}_{i j}$ e $\beta_{i j} \tilde{\Pi}_{i j}$, que fazem parte dos termos adicionais definidos nas eqs.(3.114) e 
(3.115). Embora esses termos individualmente apareçam como termos relevantes, a soma deles é efetivamente mais fraca com respeito ao coeficientes das figuras (b) e (e).

No que diz respeito à correlação $C(\omega)$, também podemos fazer uma descomposição semelhante a da $S_{11}(\omega)$ e $S_{22}(\omega)$. A fim de obter uma melhor compreensão do perfil espectral da correlação, vamos analisar a correlação de intensidade dada por $S_{12}$ (eq. 3.116), associada ao coeficientes $\nu_{i j}$. Estes coeficientes determinam a contribuição dos elementos de matriz $\tilde{\Pi}_{i j}$ empregados para calcular a correlação de intensidade da figura 3.19.(a). A contribuição efetiva é representada pelos produtos $\nu_{i j} \Pi_{i j}$, os quais são apresentados na figura 3.19.(b) e (c). Ao contrario do modelo heurístico em que a resposta da absorção e da dispersão possuem o mesmo peso para contribuir na correlação $g^{(2)}(0)$, a figura 3.19(b) mostra que, no domínio da frequência, a contribuição da parte dispersiva $\tilde{\nu}_{46}(\omega)\left[\operatorname{Re} p_{1} \operatorname{Re} p_{2}+\cdots\right]$ é significativamente maior que a da resposta de absorção $\tilde{\nu}_{35}(\omega)\left[\operatorname{Im} p_{1} \operatorname{Im} p_{2}+\cdots\right]$. Isso também mostra que a contribuição para a correlação $C(\omega)$ na eq.(3.120) depende fortemente de $\tilde{\nu}_{45}(\omega)$ associado ao produto $\operatorname{Im} p_{2} \operatorname{Re} p_{1}$, traçado na figura 3.17(d).

Além dessas diferenças entre $g^{2}(0)$ e $C(\omega)$, podemos notar que a correlação $C(\omega)$ também apresenta a contribuição dos termos $\nu_{44}(\omega)\left[\operatorname{Re}^{2} p_{1}+\cdots\right]$ e $\nu_{66}(\omega)\left[\operatorname{Re}^{2} p_{2}+\cdots\right]$, como mostra a figura 3.19(c). Os coeficientes $\nu_{33}(\omega), \nu_{55}(\omega), \nu_{34}(\omega)$ e $\nu_{56}(\omega)$ mostram uma contribuição menor com respeito aos outros coeficientes, os quais são associados ao termos $C_{12}$ na eq. (3.120). A combinação destes elementos levam a uma anti-correlação entre os campos para $|\delta| / 2 \pi<3$ MHz. No entanto, para $\delta=0$, os únicos elementos não nulos que efetivamente contribuem para a correlação entre os campos são $\tilde{\Pi}_{46}, \tilde{\Pi}_{44}$ e $\tilde{\Pi}_{66}$ (ver o gráfico interno das figuras (b) e (c)). Agora, para $|\delta| / 2 \pi \sim 3.5 \mathrm{MHz}$ o termo dispersivo $\nu_{46} \tilde{\Pi}_{46}$ toma seu máximo valor se tornando a principal contribuição para induzir uma correlação positiva entre os campos.

Por outro lado, para dessintonias $|\delta|>\Gamma=(2 \pi) 6 \mathrm{MHz}$, a competição entre os termos não nulos conduzem a uma anti-correlação entre os campos. No entanto, cabe notar que a inversão do sinal do termo $\nu_{45}(\omega)\left[\operatorname{Im} p_{2} \operatorname{Re} p_{1}+\cdots\right]$ próximo de $|\delta| \sim 5 \mathrm{MHz}$ na figura. 3.19(b), determina a inversão de sinal da correlação $C$ na figura. 3.19.(a). Este termo em particular pode ser entendido como luz sendo absorvida pelo campo $\mathbf{E}_{2}\left(\operatorname{Im} p_{2}\right)$, o qual está em ressonância $\left(\Delta_{2}=0\right)$, e que reemite luz no modo de frequência do campo $\mathbf{E}_{1}$, dependendo 


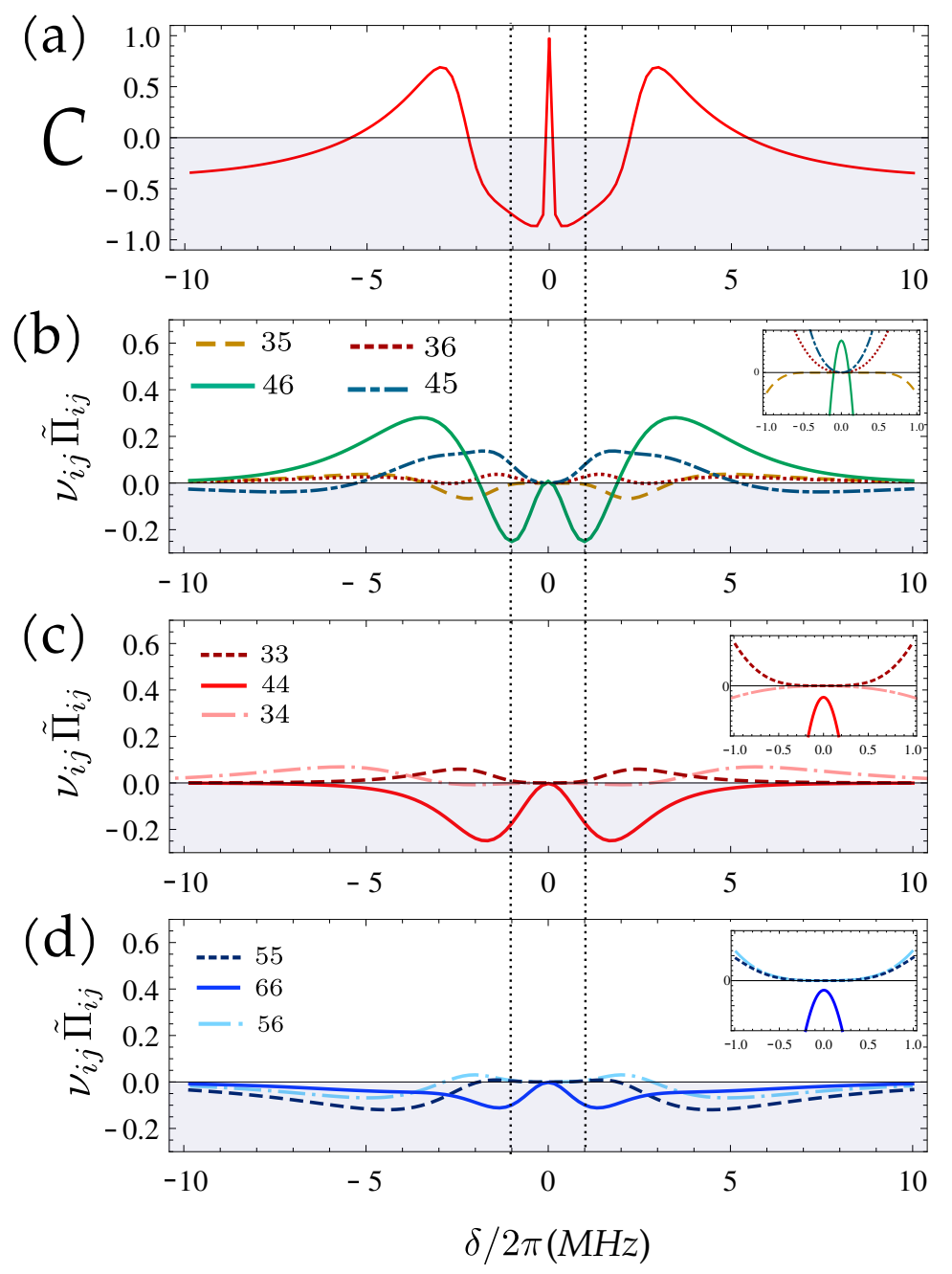

Figura 3.19: (a) Coeficiente de correlação. (b) e (c) apresentam os coeficientes $\nu_{i j}(\omega)$ associados aos elementos de matriz $\tilde{\Pi}_{i j}$ necessários para obter a densidade espectral cruzada $S_{12}(\omega)$ for $\Delta_{2}=0 e$ $\omega / 2 \pi=2 \mathrm{MHz}$. As linhas verticais representam a janela de transparência $\Delta \omega_{E I T}$.

fortemente da sua dispersão associada com $\operatorname{Re} p_{1}$. Portanto, temos transições Stokes não ressonantes que contribuem à anticorrelação. ${ }^{16}$

\footnotetext{
${ }^{16}$ As transições Stokes acontecem em sistemas atômicos de três níveis não degenerados. No caso do sistema $\Lambda$ de três níveis da figura 3.9, pela absorção de luz na transição $|1\rangle \rightarrow|3\rangle$ é emitido um fóton, denominado Stokes, na transição $|3\rangle \rightarrow|2\rangle$. No caso oposto chamaríamos de transição anti-stokes
} 


\subsubsection{Alargamento da espectroscopia de correlação devido às ressonâncias das bandas laterais}

Nesta seção investigamos a correlação de intensidade para diferentes frequências de análise. Na figura 3.20 mostramos a correlação $C(\omega)$ para $\omega / 2 \pi=2 \mathrm{MHz}, 3 \mathrm{MHz}, 4 \mathrm{MHz}$ e 5 $\mathrm{MHz}$, comparando-a com a função $g^{2}(0)$, considerando o ruído padrão de um laser de diodo $\bar{\gamma} / 2 \pi=1 \mathrm{MHz}$. Na figura 3.20(a), traçamos o coeficiente de correlação $C$ calculando os elementos $\tilde{\Pi}$ até primeira ordem em $\bar{\gamma}$, enquanto que na figura 3.20(b) eles são calculados até ordem $\bar{\gamma}^{7}$. Os perfis espectrais em geral apresentam similaridades, uma delas é o perfil da correlação no intervalo de dessintonias dentro de $3 \times \Delta \omega_{E I T}$ para todas as frequências de análise. Além disso, podemos constatar que todos mostram a mesma largura intrínseca em torno de $\delta=0$, pois ela é dada pelo inverso da coerência atômica entre os níveis fundamentais. A maior diferença entre os espectros da figura (a) e (b) é a saturação da correlação no caso linearizado (os elementos $\tilde{\Pi}_{i j}$ até primeira ordem) para $|\delta|<3 \Delta \omega_{E I T}$, enquanto que nos espectros da figura (b) não são saturados.
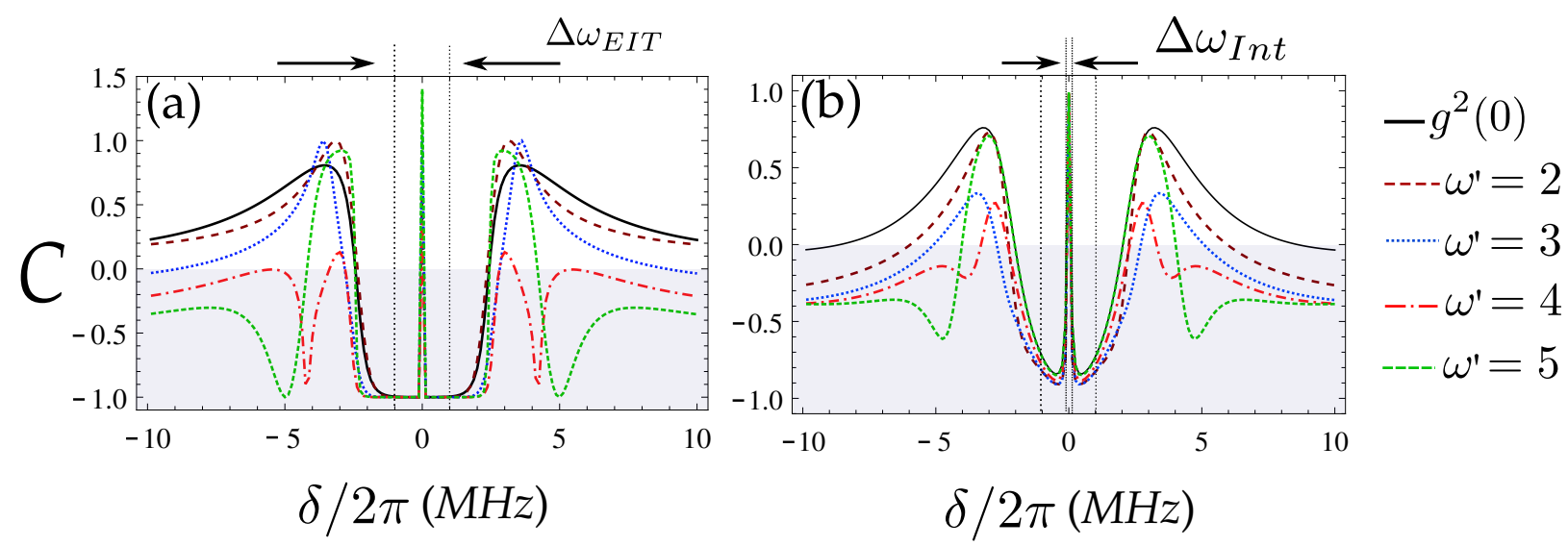

Figura 3.20: Espectro de correlação de intensidade com largura de linha $\bar{\gamma} / 2 \pi=1 \mathrm{MHz}$ expandido até (a) Primeira ordem $\bar{\gamma} e(b)$ até ordem $\bar{\gamma}^{7}$ na eq.(3.120). Para tais espectros, consideramos $\omega^{\prime}=$ $\omega / 2 \pi=2 \mathrm{MHz}, 3 \mathrm{MHz}, 4 \mathrm{MHz}$ and $5 \mathrm{MHz}$. Os parâmetros para obter os espectros são os mesmos considerados na figura 3.15. As duas linhas verticais representam a janela de transparência $\Delta \omega_{E I T}$.

Uma característica importante que notamos ao aumentar a frequência de análise, é a presença de ressonâncias quando $\delta= \pm \omega$ para $\omega / 2 \pi=4 \mathrm{MHz}$ e $5 \mathrm{MHz}$. Entretanto, para 
baixas frequências de análise, há um alargamento no perfil de anticorrelação para $\Delta \omega_{\text {Int }}<$ $|\delta|<3 \Delta \omega_{E I T}$, quando o comparamos com $g^{2}(0)$. Isto não acontece para $\omega / 2 \pi=5 \mathrm{MHz}$, pois a correlação $C$ coincide com a função $g^{2}(0)$ (em linha sólida) para $|\delta|<3 \times \Delta \omega_{E I T} \sim$

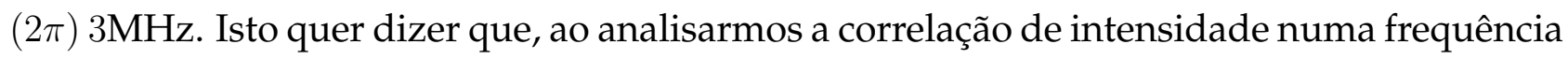
particular, as ressonâncias na frequência da banda lateral não influenciam o processo de RFRA dentro de uma banda de frequências $3 \times \Delta \omega_{E I T}$ em torno de $\delta=0$. Por outro lado, cabe notar que para qualquer frequência de análise, a largura intrínseca de $\Delta \omega_{\text {Int }}$ não é afetada. Isto garante que ao medi-la no domínio das frequências ou do tempo, ela é a mesma, sem importar a frequência de análise escolhida [43, 44]. Uma última característica relevante é a da figura (b), em que ao considerar os termos de ordem superior, as ressonâncias na frequência das bandas laterais sofrem um alargamento (especialmente para $\omega / 2 \pi=4 \mathrm{MHz}$ ) e um menor grau de anticorrelação.

Para obter uma melhor compreensão das ressonâncias nas bandas laterais decompomos a correlação em termos da resposta atômica, como foi feito na seção 3.6.2. Os coeficientes $\nu_{i j}(\omega)$ são traçados na figura 3.21 para $\omega / 2 \pi=5 \mathrm{MHz}$, em que as ressonâncias nas bandas laterais são resolvidas. Como no caso da figura 3.19, a principal contribuição para a correlação é dada pelos termos dispersivos $\tilde{\Pi}_{46}$ e $\tilde{\Pi}_{45}$ para $\delta \neq \omega$. No entanto, nas ressonâncias $\delta= \pm \omega$, eles contribuem em menor medida que os termos $\tilde{\Pi}_{44}$ e $\tilde{\Pi}_{66}$, os quais são negativos e conduzem a uma anti-correlação entre os campos.

Assim fizemos a decomposição em termos dos elementos de matriz $\tilde{\Pi}_{i j}$ e dos coeficientes $\nu_{i j}(\omega)$ apresentados nas figuras 3.17 e 3.21 , respectivamente. Eles mostram a contribuição independente do processo RF-RA quando a dessintonia entre as portadoras é $|\delta|<3 \times \Delta \omega_{E I T}$ $\operatorname{com} \omega / 2 \pi=4 \mathrm{MHz}$ e $5 \mathrm{MHz}$. Entretanto, para $\omega / 2 \pi \leq 3 \mathrm{MHz}$, as ressonâncias na frequência das bandas laterais não são resolvidas, alargando o perfil espectral da correlação no domínio da frequências para $\Delta \omega_{\text {Int }}<|\delta|<\Delta \omega_{E I T}$.

\section{Conclusões do capítulo}

Fizemos uma revisão detalhada dos dois principais modelos propostos para determinar a correlação de intensidade de dois campos interagindo com um átomo de três níveis em 

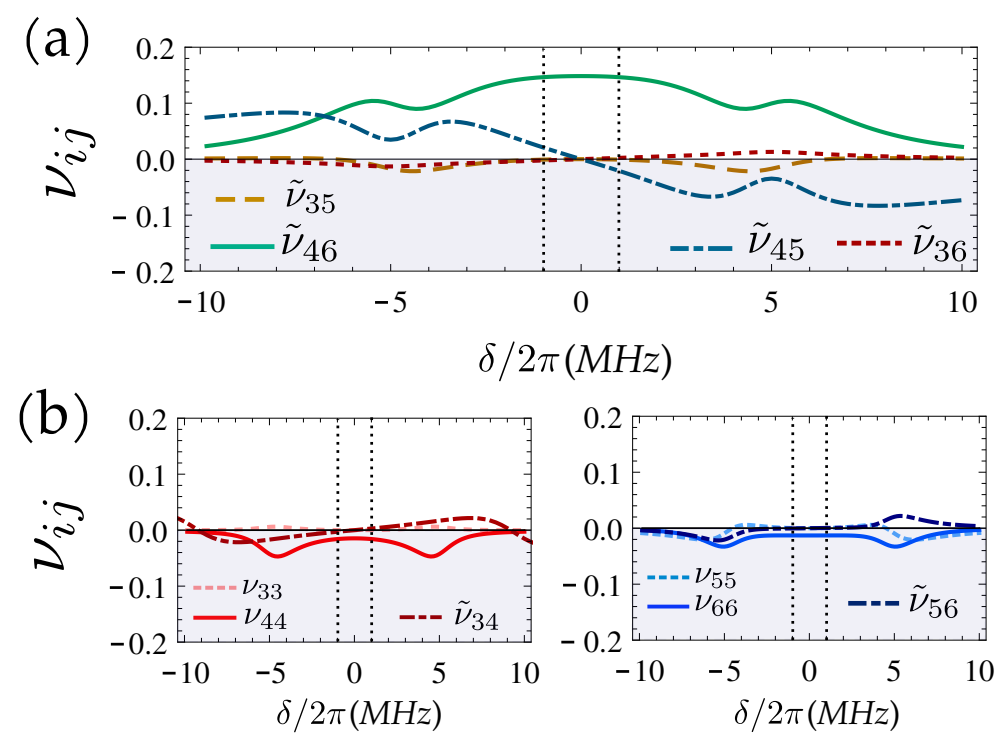

Figura 3.21: Coeficientes $\nu_{i j}(\omega)$ que modulam os elementos de matriz $\tilde{\Pi}_{i j}$ para obter uma densidade espectral cruzada $S_{12}(\omega)$, considerando os parâmetros $\Delta_{2}=0$ e $\omega / 2 \pi=5 \mathrm{MHz}$.

condição de EIT. Na primeira parte do capítulo mostrou-se que no domínio do tempo é possível abordar a função de correlação normalizada $g^{(2)}(0)$ mediante um modelo heurístico. Ele permite fazer uma análise simples da correlação em termos da dispersão e absorção dos átomos. Posteriormente, revisou-se a solução da dinâmica estocástica atômica empregando o cálculo de Itô e adotado até então na pequisa do laboratório. Foram analisadas as principais características de ambos os modelos.

Na terceira parte do capítulo, foi apresentado então o modelo perturbativo proposto para determinar a correlação entre os campos. Esse modelo se apresenta como um dos principais resultados da minha pesquisa durante o meu doutorado. A partir dele podemos estabelecer uma relação entre a função de correlação $C(\omega)$ e os elementos de dispersão e absorção atômica. Inclusive mostrou-se que a correlação no domínio da frequência é resultado de uma transformação matricial das covariâncias atômicas, onde a contribuição das bandas laterias e da portadora ficam claramente discriminadas. Além disso, o modelo perturbativo deixa em evidência o papel da largura de banda dos campos na dinâmica atômica. Mostrouse que para campos de luz que apresentam um excesso de ruído de fase baixo, a correlação 
à ordem $\epsilon^{2}$ é suficiente para descrever os sistema. No entanto, ao considerar luz com excesso de ruído comparável à largura natural do meio atômico, os termos de ordem superior devem ser levados em conta para ter uma descrição completa da correlação. Desta forma, a solução perturbativa permite dar uma interpretação em termos de parâmetros físicos sobre a resposta atômica para analisar o espectro de correlação dos feixes no domínio das frequências. 


\section{CAPÍTULO 4 \\ LRESULTADOS EXPERIMENTAIS DA ESPECTROSCOPIA DE CORRELAÇÃO EM CONDIÇÃO DE EIT}

4.1 Montagem experimental . . . . . . . . . . . . . . . 124

4.2 Aplicando dessintonia de um fóton na espectroscopia de correlação . . . . 136

4.3 Forças de espalhamento na espectroscopia de correlação . . . . . . . . . . 151

Fizemos uma descrição teórica da correlação de dois campos de luz interagindo com átomos com dessintonias próximas à ressonância de EIT. Neste capítulo, mostraremos os resultados experimentais para a espectroscopia de correlação entre lasers de diodo, interagindo com átomos frios de Rubídio em condição de EIT. Na primeira parte, mostraremos a montagem experimental, onde descrevemos brevemente as características da nossa amostra de átomos frios e do nosso sistema de deteção. Na segunda parte, mostraremos os espectros de correlação em condição de EIT para duas experiências em particular: o regime dessintonizado, onde o espectro de correlação resolve as ressonâncias nas frequências das bandas laterais e o regime dinâmico, onde analisaremos o efeito de "histerese"na espectroscopia de correlação. 


\subsection{Montagem experimental}

A figura 4.1 mostra a montagem experimental usada para medir a correlação dos campos em condição de EIT. No experimento usamos lasers de diodo com cavidade externa em configuração Littrow [81, 82], cujo comprimento de onda é de 780,246nm, compatível com a linha D2 do Rubídio, e representado pela linha vermelha. A largura de linha do espectro de emissão do laser é de 1,2 \pm 0,2 MHz. Após passar pelo isolador ótico, uma pequena porção da luz é usada para fazer uma espectroscopia de absorção saturada (Abs. Sat.)[69, 74] em células de vapor de Rubídio. Esta espectroscopia atua como referência para estabilizar a frequência do laser que interage com a nuvem de átomos frios numa transição específica. $O$ restante da luz passa por dois prismas anamórficos (PR) para corrigir o perfil de intensidade transversal elíptico presente desde a sua saída. Depois dos prismas, o feixe passa por um telescópio. A frente de onda que sai do laser possui uma pequena divergência, de modo que o telescópio diminui a cintura do feixe à metade, e corrige a frente de onda, diminuindo a divergência durante o percurso pelos espelhos, até a câmara onde estão os átomos frios. Após passar pelo telescópio, separamos o feixe através de um Polarizing Beam-Splitter (PBS), que vai dividir o feixe em componentes de polarização vertical $(\mathrm{V})$ e horizontal $(\mathrm{H})$. Estas duas componentes serão os campos $\mathbf{E}_{1}$ e $\mathbf{E}_{2}$, que acoplam três níveis para obter EIT.

Antes de interagir diretamente com os átomos frios, ambos os feixes são acoplados com Moduladores Acusto-Óticos (AOM sigla em inglês), que mediante ondas de Rádio Frequência (RF), modulam a frequência e a amplitude do laser [83]. $\mathrm{O}$ fato de manipular a frequência de cada um dos feixes de forma independente, permite introduzir dessintonias $\Delta_{1}$ e $\Delta_{2}$ em cada um dos campos com respeito à transição atômica. Na saída dos AOMs, os campos passam por lâminas de onda $\lambda / 4$, transformando a polarização linear $\mathrm{V}$ e $\mathrm{H}$ dos campos para polarizações circulares $\sigma+\mathrm{e} \sigma-$, incidindo na nuvem atômica com um ângulo $\theta \sim 2^{\circ}$. Assim, nessa geometria quase co-linear, os campos acoplam a transição ${ }^{5} S_{1 / 2} F=1 \rightarrow{ }^{5} P_{3 / 2} F=1$ do $\mathrm{Rb}^{87}$ na configuração $\Lambda$-EIT, como mostra a figura 4.2.(a).

Após a luz interagir com os átomos, cada componente de polarização é medida por fotodetectores. O sinal dos fotodetetores é demodulado numa certa frequência $\omega / 2 \pi$, e a sua 


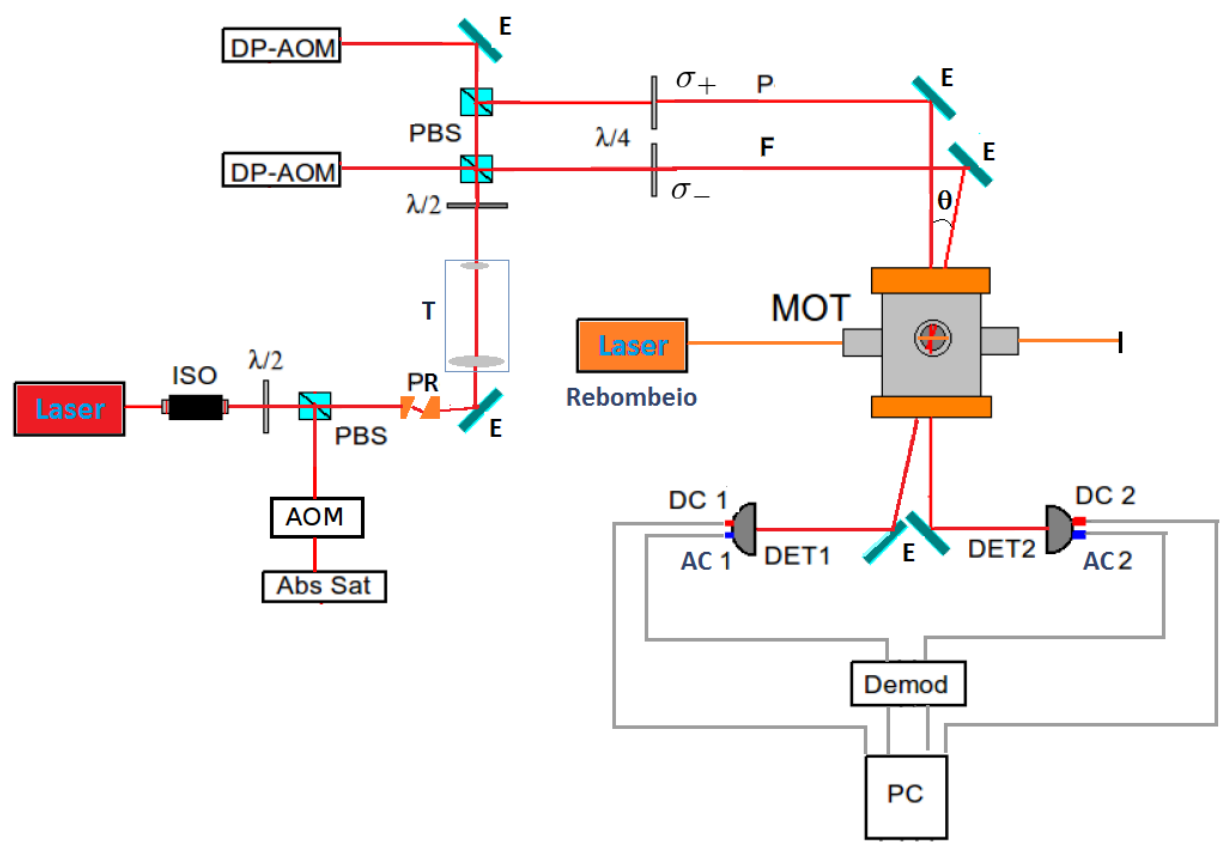

Figura 4.1: Montagem Experimental. ISO: Isolador ótico. $\lambda / 2$ : Lâmina de meia onda. $\lambda / 4$ : Lâmina de quarto de onda. PBS: Polarizer Beam Splitter. AOM: Modulador acusto-ótico. Abs. Sat.:Espectroscopia de absorção Saturada. PR:Prismas anamórficos. E: Espelho. T: Telescópio. DET: Detetor. MOT: Armadilha magneto-ótica. Demod: Demodulador. PC:Computador.

saída é conectada diretamente a uma placa de aquisição para armazenar a informação no computador (adiante farei a descrição deste sistema de deteção).

A partir do sinal demodulado das flutuações de intensidade da luz $\delta I(\omega)$, podemos determinar o coeficiente de correlação $C(\omega)$. A espectroscopia de correlação de intensidade é feita colocando um valor fixo numa das dessintonias, por exemplo $\Delta_{1}$, e aplicando uma varredura linear no tempo na dessintonia $\Delta_{2}$. Para fazer esta espectroscopia, precisamos de um outro laser (linha laranja) como bombeio ótico, que é o encarregado de transferir a população dos níveis ${ }^{5} S_{1 / 2} F=2$, em que é preparada a nuvem atômica, para o estado ${ }^{5} S_{1 / 2} F=1$. Isto é feito acoplando o laser de bombeio na transição ${ }^{5} S_{1 / 2} F=2 \rightarrow{ }^{5} P_{3 / 2} F=2$, tal que pela emissão espontânea, os átomos são transferidos ao ${ }^{5} S_{1 / 2} F=1$, como mostra a figura 4.2.(b). Uma vez transferida a população atômica para o nível $F=1$, fazemos a espectroscopia de correlação em EIT. 


\subsection{1 Átomos frios}

O experimento usa uma amostra de átomos frios a uma temperatura de $\sim 450 \mu \mathrm{K}$, obtida a partir de um processo de resfriamento por laser numa armadilha magneto-ótica (MOT, sigla em inglês)[41, 42], dentro de uma câmara de vácuo, a uma pressão de $10^{-8}$ Torr. Este resfriamento é feito acoplando três pares de laser na transição ${ }^{5} S_{1 / 2}(F=2) \rightarrow{ }^{5} P_{3 / 2}\left(F^{\prime}=3\right)$ do rubídio $87\left({ }^{87} \mathrm{Rb}\right)$, como mostra a figura 4.2.(b). De acordo com a eq.(2.23), cada par exerce forças de espalhamento sobre os átomos, de tal forma que a força resultante é proporcional a $-\alpha \mathbf{v}$, onde $\alpha$ é uma constante determinada pela dessintonia e intensidade dos campos. Isto constitui então uma força de amortecimento sobre a velocidade dos átomos. Para que isto ocorra, é necessário manter a população atômica no nível ${ }^{5} S_{1 / 2}(F=2)$. Portanto, acoplamos um laser externo na transição ${ }^{5} S_{1 / 2}(F=1) \rightarrow^{5} P_{3 / 2}\left(F^{\prime}=2\right)$, para rebombear aqueles átomos que eventualmente decaem para ${ }^{5} S_{1 / 2}(F=1)$.
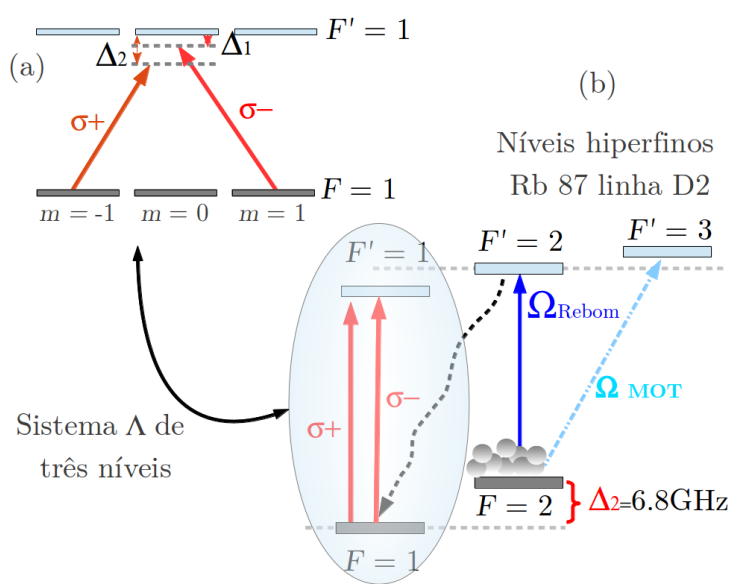

(b)

Níveis hiperfinos Rb 87 linha D2 $F^{\prime}=2 \quad F^{\prime}=3$

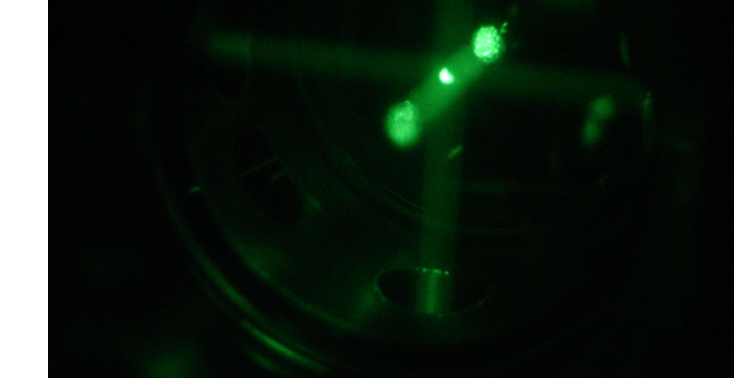

Figura 4.2: (a) Configuração $\Lambda$ dos feixes $\sigma+e \sigma-$, acoplando a transição $F=1 \rightarrow F^{\prime}=1$. (b) Estrutura de níveis hiperfinos na linha $\mathrm{D} 2$ do ${ }^{87} \mathrm{Rb}$. $\Omega_{M O T}$ é a frequência de Rabi dos feixes usados para resfriamento. $\Omega_{\text {rebom }}$ corresponde ao rebombeio usado para transferir a população do estado $F=2 \rightarrow F=1$, por meio do estado excitado $F^{\prime}=2$. (c) Imagem com visor infravermelho da nuvem de átomos frios de Rubídio na MOT.

São empregadas também duas bobinas em configuração anti-Helmoltz para gerar o campo magnético necessário para o aprisionamento na MOT. Elas estão constituídas de 550 espiras cada, com uma corrente de $2.00 \mathrm{~A}$, tal que o gradiente de campo é de $\sim 20$ Gauss $/ \mathrm{cm}$. O 
número médio de átomos na nuvem é de $10^{7} \mathrm{e}$ a densidade ótica na transição de resfriamento é $\alpha L \sim 2$. A figura 4.2.(c) mostra os três pares de feixes contrapropagantes na câmara de vácuo, e no centro uma nuvem de átomos frios de aproximadamente $3 \mathrm{~mm}$.

A figura 4.3 mostra a mesa ótica onde temos o sistema de resfriamento de átomos e o sistema de deteç̧ão de luz para os experimentos de espectroscopia. A sincronização do experimento para o controle dos átomos e a aquisição de dados é feita mediante rotinas de controle programadas na interface de Labview.

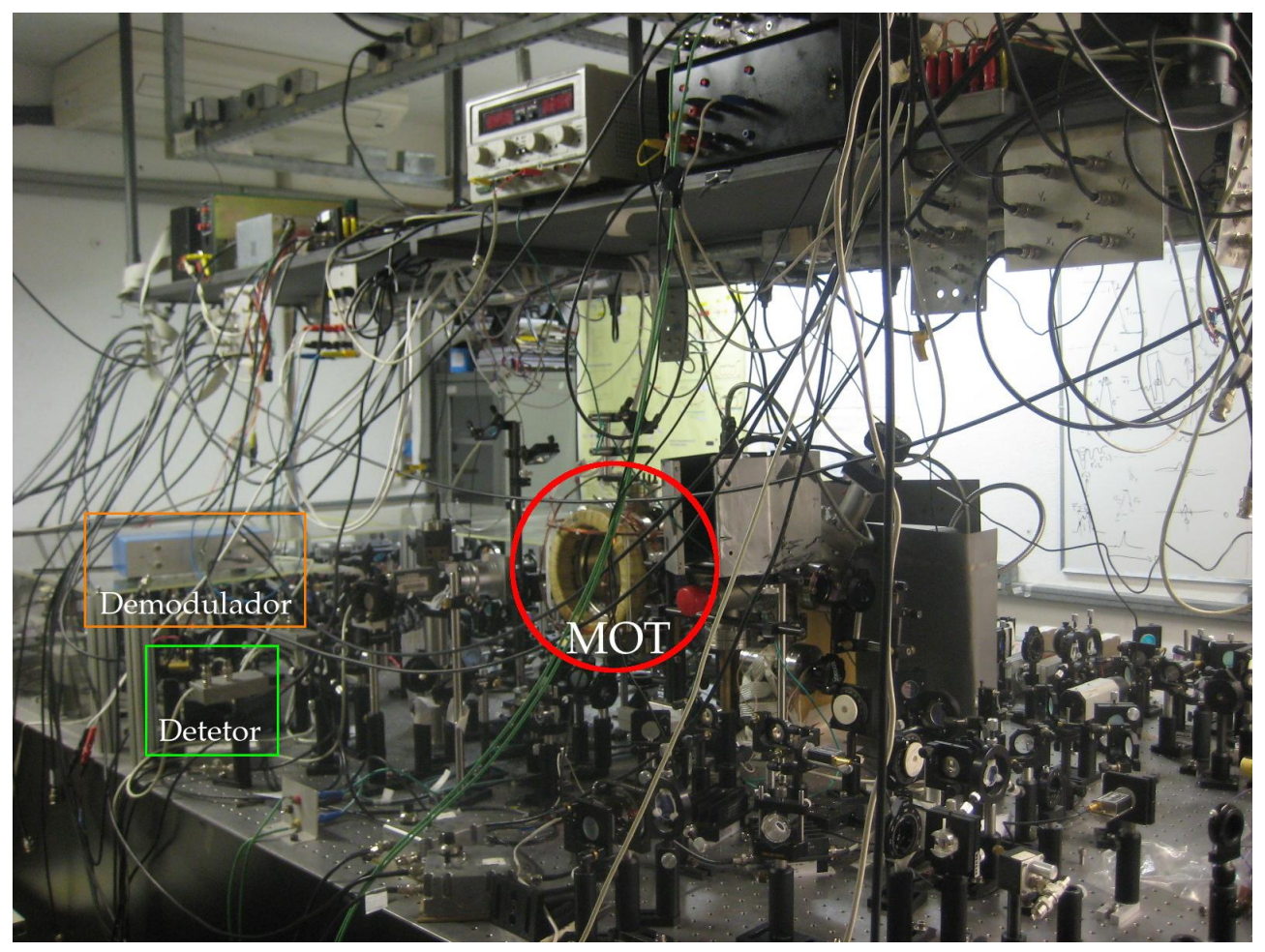

Figura 4.3: Mesa ótica para experimentos de espectroscopia de correlação em EIT com átomos frios

\subsubsection{Deteção da luz}

Em nosso experimento usamos fotodiodos para detectar os feixes de luz intensos que interagem com os átomos frios. Eles são sensíveis unicamente à intensidade porque funcionam a partir do efeito fotoelétrico. A intensidade dos campos é convertida então em uma cor- 
rente elétrica, chamada fotocorrente, que é proporcional à função de correlação de Glauber $[84,85]$

$$
\langle i(t)\rangle=K\left\langle E(\mathbf{r}, t)^{(-)} E(\mathbf{r}, t)^{(+)}\right\rangle
$$

onde $K$ leva em conta qualquer contribuição de amplificação introduzida pela eletrônica de detecção.

Para medir a correlação de intensidades no domínio da frequência, empregamos várias etapas eletrônicas no sinal da fotocorrente. A primeira etapa é feita no interior do detector, onde a fotocorrente é dividida em sinal DC e sinal $\mathrm{AC}$, onde este último é conetado a um amplificador de trans-impedância, como mostra a figura 4.4. O sinal DC dá acesso ao sinal médio da intensidade detectada $\bar{I}$, e ao sinal AC permite medir o ruído da fotocorrente. Após ser amplificado, o sinal AC passa para uma etapa de demodulação. O demodulador mistura o sinal de entrada com o sinal de um Oscilador Local (LO) com frequência $\omega_{a}$, produzido por um gerador de funções Hewlett Packard (HP). O sinal demodulado passa por um amplificador com um filtro passa-baixa de $300 \mathrm{kHz}$, produzindo assim, na sua saída, uma imagem do sinal eletrônico na frequência de análise $\omega_{a}$ em baixa frequência, com uma largura de banda de $600 \mathrm{kHz}$. Desta forma, obtemos as flutuações proporcionais a $\delta I\left(\omega_{a}\right)$ para cada um dos feixes de luz, com o qual podemos determinar o coeficiente de correlação da eq.(3.90).

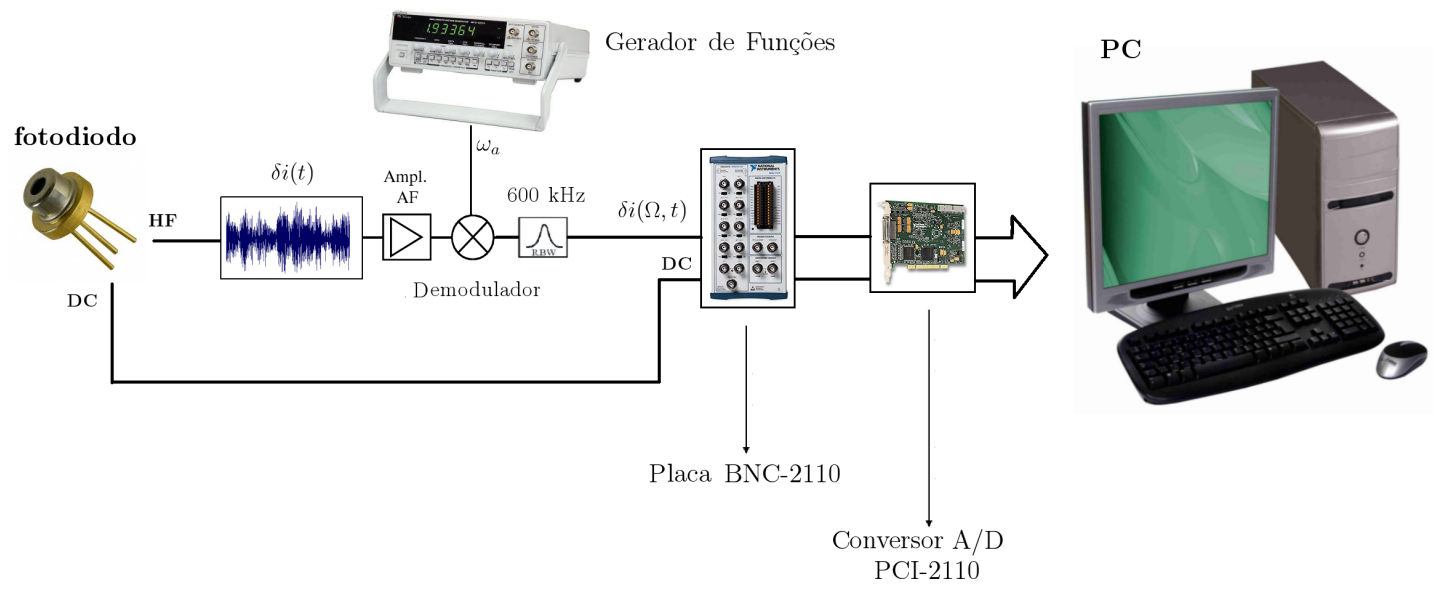

Figura 4.4: Sistema de deteção e de aquisição de dados 
Este esquema de demodulação é padrão no laboratório [53, 86, 87], pois permite adquirir tanto o sinal DC, como o sinal de alta frequência ao mesmo tempo, mediante uma placa de aquisição de dados da National Instruments (modelo BNC-2110), conectada a uma placa conversora A/D (análogo-digital) da mesma empresa (modelo PCI-2110), que adquire os dados diretamente no computador (PC) mediante o programa LabView.

\subsubsection{Análise do ruído do Laser de diodo mediante rotação de elipse}

Como foi discutido ao longo do capítulo 1 e o capítulo 3, uma das características principais do fenômeno que estamos estudado neste trabalho, é o excesso de ruído na fase do laser de diodo (ver figura 4.5.(a)). Portanto, nesta seção mostraremos que essa condição é satisfeita antes de interagir com os átomos e vamos compará-la com o caso de um laser sem excesso de ruído, como é o Titânio safira, cujo estado corresponde ao de um estado coerente (ver 4.5.(b)). Para isto é necessário aplicar técnicas de homodinagem para medir o ruído de fase do laser, pois como foi mencionando anteriormente, os detetores são sensíveis somente à intensidade da luz. Para isto aplicaremos a técnica de rotação de elipse introduzida por A. Villar em [88], que é aplicada de forma padrão no laboratório. Ela permite medir o ruído das quadraturas de amplitude e fase da luz mediante o uso de uma cavidade ótica. Faremos aqui uma breve descrição da técnica, que pode ser encontrada nas referências [53, 86, 88], as quais fazem uma ampla descrição desta técnica.

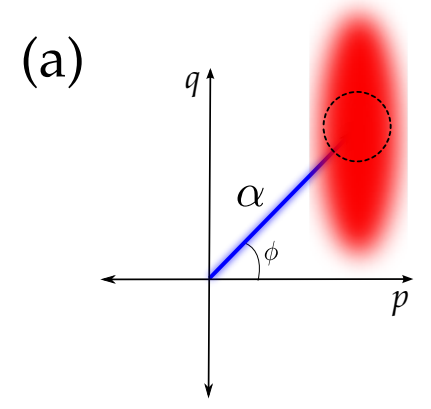

(b)

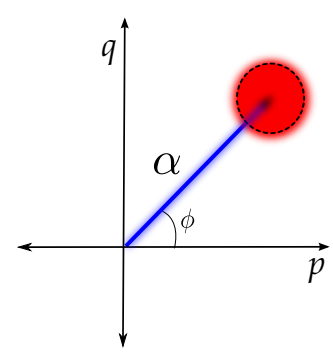

Figura 4.5: Diagramas de Fresnel representando o espaço de fase quântico. (a) Estado com ruído de amplitude igual ao shot-noise, mas com excesso de ruído na sua quadratura de fase. (b) Estado coerente.

Como foi discutido na seção 1.2, a amplitude de um campo de luz $\alpha(t)$ que apresenta 
flutuações de intensidade pode ser escrito como $\alpha(t)=\bar{\alpha}+\delta \alpha(t)$, em que $\bar{\alpha}$ e $\delta \alpha(t)$ representam o valor médio e as flutuações de intensidade no tempo,respectivamente. Um feixe intenso, como os que são usados nas experiências deste trabalho, satisfaz a condição $|\delta \alpha(t)| \ll \bar{\alpha}$. Portanto, a intensidade do campo pode ser escrita como

$$
I(t)=\alpha^{*}(t) \alpha(t) \approx \bar{I}+\bar{\alpha}^{*} \delta \alpha(t)+\bar{\alpha}+\delta \alpha^{*}(t)
$$

de modo que a flutuação de intensidade é dada por

$$
\delta I(t)=\bar{\alpha}^{*} \delta \alpha(t)+\bar{\alpha} \delta \alpha^{*}(t)
$$

Dado que a amplitude $\bar{\alpha}$ é um número complexo, temos então que $\bar{\alpha}=|\bar{\alpha}| e^{i \phi}$. Ao normalizar a flutuação de intensidade 4.3 pelo valor absoluto da amplitude do campo, obtemos a flutuação na quadratura de amplitude

$$
\delta p(t)=e^{-i \varphi} \delta \alpha(t)+e^{i \varphi} \delta \alpha^{*}(t)
$$

de modo que $\delta I(t)=|\bar{\alpha}| \delta p(t)$. De acordo com a eq.(1.72), a flutuação da quadratura de fase será definida então como

$$
\delta q(t)=-i\left[e^{-i \varphi} \delta \alpha(t)-e^{i \varphi} \delta \alpha^{*}(t)\right]
$$

Ao aplicar a transformada de Fourier nas flutuações de amplitude e fase, temos então

$$
\begin{array}{r}
\delta p(\nu)=e^{-i \varphi} \delta \alpha(\nu)+e^{i \varphi} \delta \alpha^{*}(-\nu) \\
\delta q(\nu)=-i\left[e^{-i \varphi} \delta \alpha(\nu)-e^{i \varphi} \delta \alpha^{*}(-\nu)\right]
\end{array}
$$

o que define as flutuações de amplitude e fase no domínio das frequências, adotado ao logo deste trabalho, como batimentos entre a frequência ótica da portadora e as bandas laterais $\pm \nu$. A técnica de rotação de elipse consiste em acoplar a luz com uma cavidade ótica, como mostra a figura 4.6.(a), de modo que, ao varrer a frequência de ressonância da cavidade com 
respeito à frequência do campo, pela interferência entre os campos intra-cavidade, na sua saída, o feixe refletido permite medir a conversão do ruído de fase $\delta q$ em ruído de amplitude $\delta p$ para as frequências da portadora e das bandas laterias. A figura 4.6.(b) mostra o perfil típico da reflexão do campo em função da dessintonia da cavidade com respeito à frequência do laser.

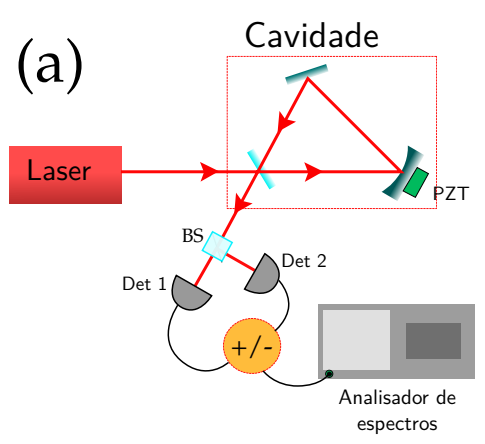

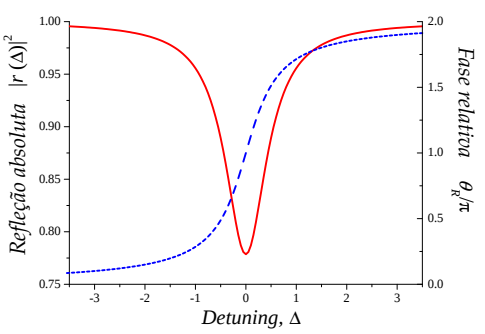

(b)

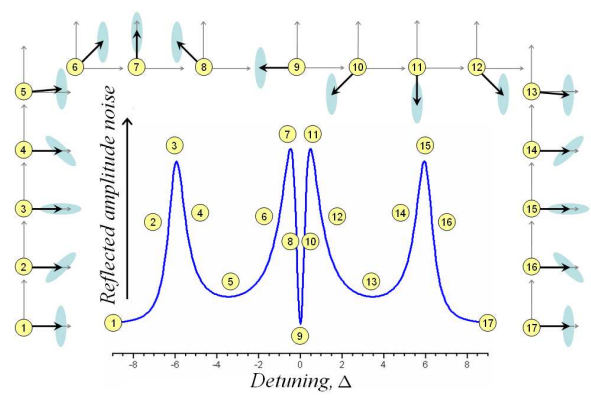

(c)

Figura 4.6: (a)Montagem experimental para realizar a técnica de rotação de elipse. (b) Coeficiente de reflexão e a defasagem do campo refletido na cavidade em função da dessintonia entre a portadora e a ressonância da cavidade, obtido da ref. [88]. (c) Rotação da elipse de ruído em função da dessintonia entre a portadora e a ressonância da cavidade, obtido da ref. [88].

No caso em que a luz apresenta excesso ou compressão de ruído ou compressão em uma das suas quadraturas, o ruído do campo representado no diagrama de Fresnel por uma elipse como a da figura 4.5.(a). Para ilustrar fenomenologicamente a conversão de ruído aplicando esta técnica, mostramos a figura 4.6 tomada da referência [88]. Nela mostramos como a elipse de ruído rota conforme a dessintonia da cavidade é modulada em um intervalo de frequências específico, considerando a frequência $\nu^{\prime}=6$, sendo $\nu^{\prime}$ a frequência das bandas laterias $\nu$ normalizada pela largura de banda da cavidade. Em particular temos que, no ponto de dessintonia 1 onde a cavidade está altamente dessintonizada com relação ao campo, o ruído de amplitude toma seu mínimo valor. Porém, à medida que a dessintonia da cavidade se aproxima da frequência da banda lateral $\nu=-6$, os pontos 2, 3 e 4 mostram o aumento no ruído de amplitude devido à rotação da elipse em um ângulo de $\pi$. Assim acontece a rotação de elipse quando uma das bandas laterais passa pela ressonância da cavidade. Posteriormente, para $|\Delta|<3$, a portadora é ressonante com a cavidade, enquanto que as bandas laterais estão fora da ressonância. Portanto, a portadora sofre uma rotação de $2 \pi$ 
passando pelos pontos 5 a 13 de dessintonia. Finalmente, para dessintonias $\Delta>3$, os pontos 13 a 17 mostram a rotação de elipse quando a banda lateral $\nu^{\prime}=6$ entra em ressonância com a cavidade. Desta forma temos a transformação de ruído de fase para ruído de amplitude, o que permite caracterizar completamente o estado do laser. A resolução na rotação da elipse entre as bandas laterais e a portadora depende da relação $\nu>\sqrt{2} \Delta \nu_{c}$, onde $\Delta \nu_{c}$ representa a largura de banda da cavidade.

Aplicamos esta técnica para o laser de diodo, que será empregado nas experiências com os átomos. Para isto usamos uma cavidade ótica com Finesse de 132(6) e largura de banda $\Delta \nu_{c}=15(1) \mathrm{MHz}$. Na sua saída, a luz passa por uma deteção balanceada como mostra a figura 4.6.(a), a qual é descrita na seção 1.4.1. A figura 4.7.(a) mostra o espectro de frequências do laser que obtive no analisador de espectros ${ }^{1}$, em que o ruído eletrônico é traçado em linha verde, e o ruído da soma e da subtração das fotocorrentes são traçados em cor azul e vermelha, respectivamente. O laser de diodo já apresenta um excesso de ruído de amplitude, pois o ruído da somas dos sinais chega a ter quase $10 \mathrm{~dB}$ de diferença com respeito ao shotnoise, para algumas frequências de análise. Para fazer a rotação de elipse, medimos a feixe refletido enquanto fazemos uma varredura na dessintonia da cavidade em um intervalo de tempo de $0.45 \mathrm{~s}$. A figura 4.7.(b) mostra os perfis típicos de uma rotação de elipse para as três frequências de análise $\nu=20,25$ e $30 \mathrm{MHz}$ que satisfazem a condição $\nu \geq \Delta \nu_{c}$. Os espectros da parte superior mostram, em laranja e marrom, o sinal DC em cada um dos detetores, traçando um perfil típico da reflexão quando a luz passa pela ressonância da cavidade. $\mathrm{Na}$ parte inferior são apresentados os ruídos da soma, da subtração e o ruído eletrônico. Além da diferença de quase $20 \mathrm{~dB}$ entre o ruído do laser e o shot-noise em torno da ressonância da cavidade, podemos observar a rotação de elipse nas ressonâncias das bandas laterias espectralmente independentes da portadora para as frequências de $24 \mathrm{MHz}$ e $30 \mathrm{MHz}$, como é indicado pelas setas. Isto mostra claramente que o laser de diodo apresenta um excesso de ruído na sua quadratura de fase de $\sim 20 \mathrm{~dB}$, detectado mediante a rotação da elipse de ruído.

\footnotetext{
${ }^{1}$ Para esta medida não foi empregado o sistema de demodulação descrito na seção anterior. Para efeitos de comparação com o Titânio Safira, empregamos o Analisador de espectros que atinge frequências maiores. Ambos os sistemas permitem caracterizar de forma equivalente qualquer sinal na sua entrada.
} 


\section{(a)}

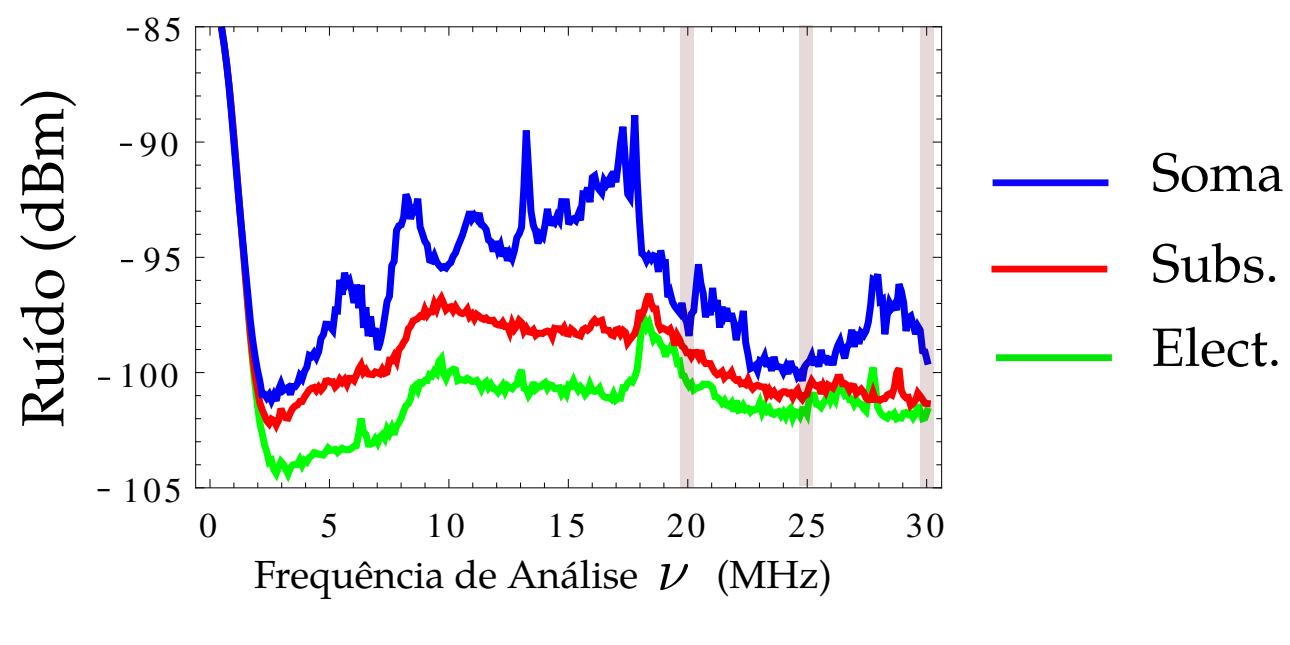

(b)
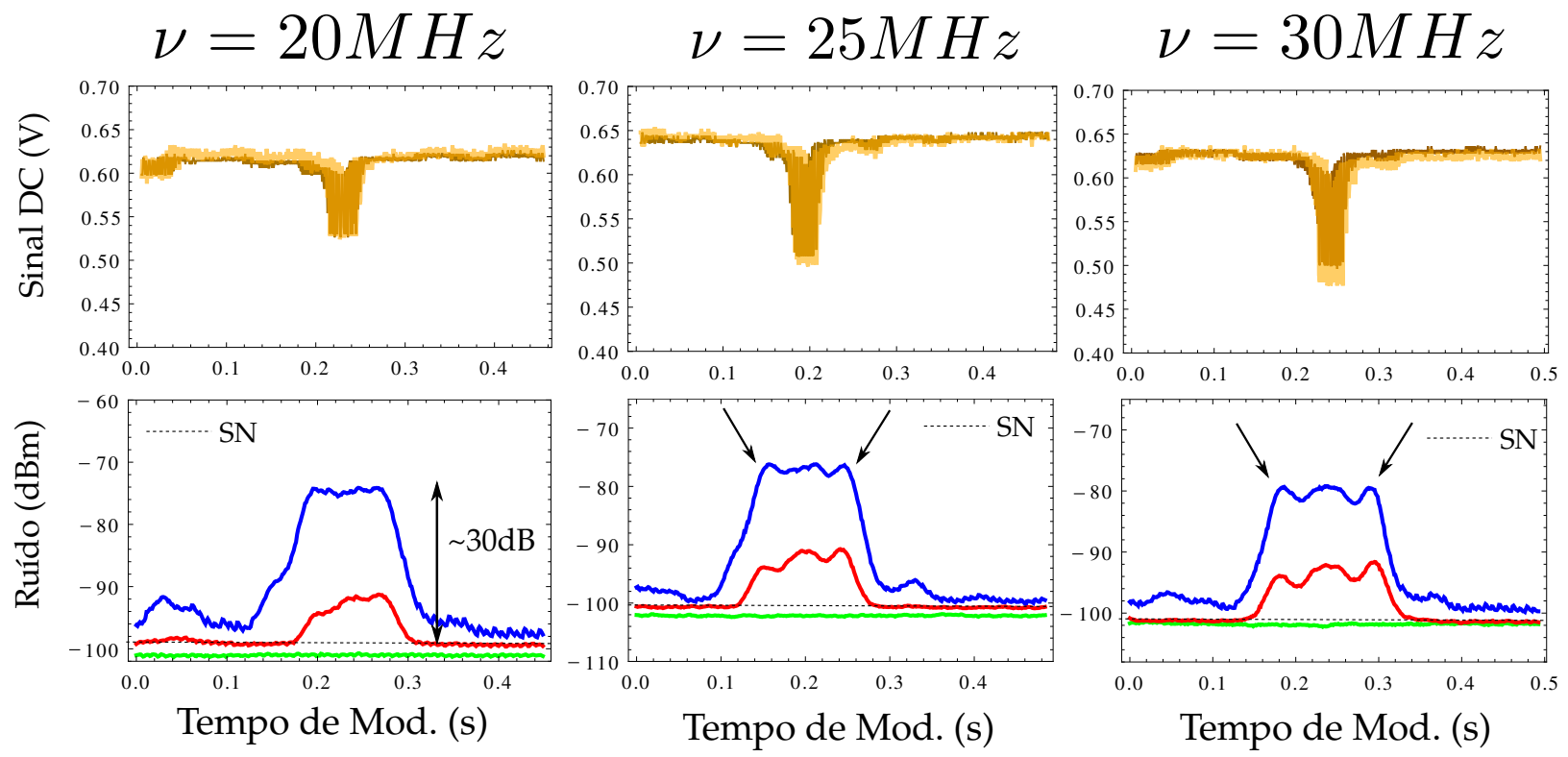

Figura 4.7: (a) Espectro de ruído do laser de diodo para uma potência $P=1.227(2) \mathrm{mW}$. (b) Rotação de elipse para as frequências de análise $\nu=20 \mathrm{MHz}, 25 \mathrm{MHz}$ e $30 \mathrm{MHz}$. SN:shot-noise

Para complementar esta análise, decidi contrastar este resultado com o caso de feixe de luz que não apresenta tal excesso de ruído. Vamos analisar o resultado para a rotação de ruído do laser de Titânio safira, pois ele é um laser que a principio, é descrito por um estado coerente. A figura 4.8.(a) mostra o espectro de ruído de amplitude do Titânio safira. Podemos notar que ele não apresenta excesso de ruído na sua quadratura de amplitude, pois o ruído da soma dos sinais coincide com shot-noise para a maioria das frequências de 
análise, como é esperado. Isto é claramente diferente do laser de diodo, se compararmos o espectro de ruído com o da figura 4.7.(a). A figura 4.8.(b) mostra o ruído de amplitude do laser à medida que é feita a rotação das quadraturas para as três frequências de análise $\nu=15,21$ e $30 \mathrm{MHz}$, das quais somente as duas últimas satisfazem a condição $\nu \geq \Delta \nu_{c}$. Os espectros da parte superior mostram, em laranja e marrom, o sinal DC em cada um dos detectores, traçando um perfil típico da reflexão quando a luz passa pela ressonância da cavidade. Na parte do centro mostramos o ruído da soma, da subtração e o ruído eletrônico. Claramente se observa que o ruído da soma se mantém muito próximo do ruído de shotnoise sem apresentar um perfil de rotação de elipse. As figuras da parte inferior mostram com maior precisão que o ruído do laser acompanha o perfil de reflexão da cavidade. Para as frequências de $21 \mathrm{MHz}$ e $30 \mathrm{MHz}$, a soma apresenta uma pequena discrepância com o shotnoise para as dessintonias próximas da ressonância que não ultrapassa um valor de $0.5 \mathrm{~dB}$, mas nada comparável ao excesso de ruído de um laser de diodo. Portanto, mostramos que o laser de titânio safira apresenta níveis de ruído nas suas quadraturas de amplitude e fase compatíveis com as de um campo em estado coerente.

Portanto mostramos, mediante a técnica de rotação de elipse, que o laser de diodo evidentemente apresenta excesso de ruído na sua quadratura de fase. Assim garantimos que o nosso sistema experimental satisfaz as condições que são levadas em conta nos modelos teóricos descritos no capitulo 3.

\subsubsection{Sequência temporal do experimento}

Retornemos às características do nosso sistema atômico e à sua preparação para o experimento. Dado que o nosso sistema $\Lambda$-EIT é definido a partir dos níveis hiperfinos $\left|F, m_{F}\right\rangle$ dos átomos de rubídio, os quais são sensíveis à presença de campos magnéticos, é necessário sincronizar temporalmente a etapa de preparação dos átomos e a etapa de medida, onde se obtém os espectros de correlação. A sequência temporal para fazer a espectroscopia em função de $\delta=\Delta_{1}-\Delta_{2}$ se mostra na figura 4.9. Os átomos são resfriados durante 1 segundo na transição $F=2 \rightarrow F^{\prime}=3$, como mostra figura 4.2.(b). Posteriormente desligamos o 
(a)

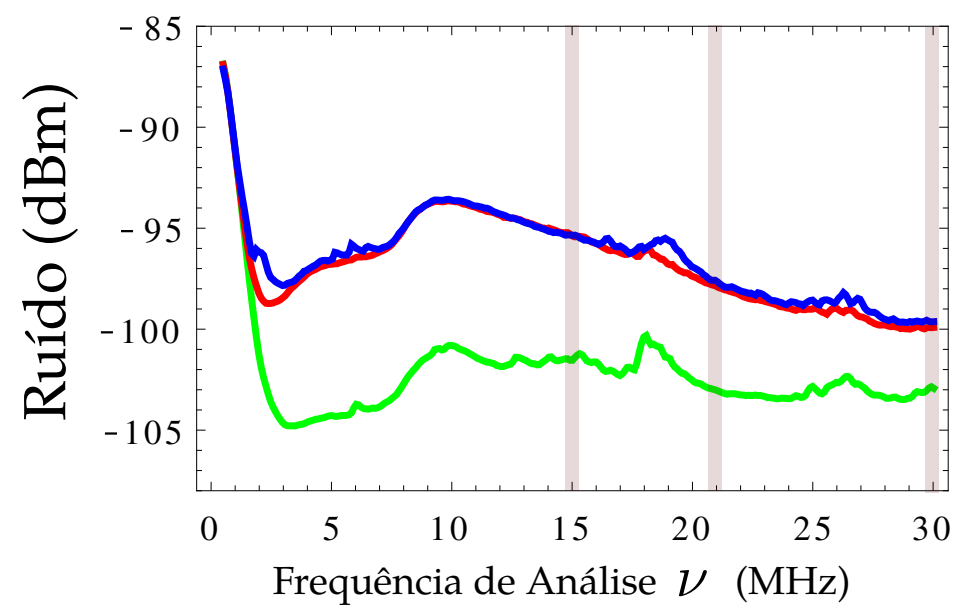

\section{— Soma}

- Subs.

Elect.
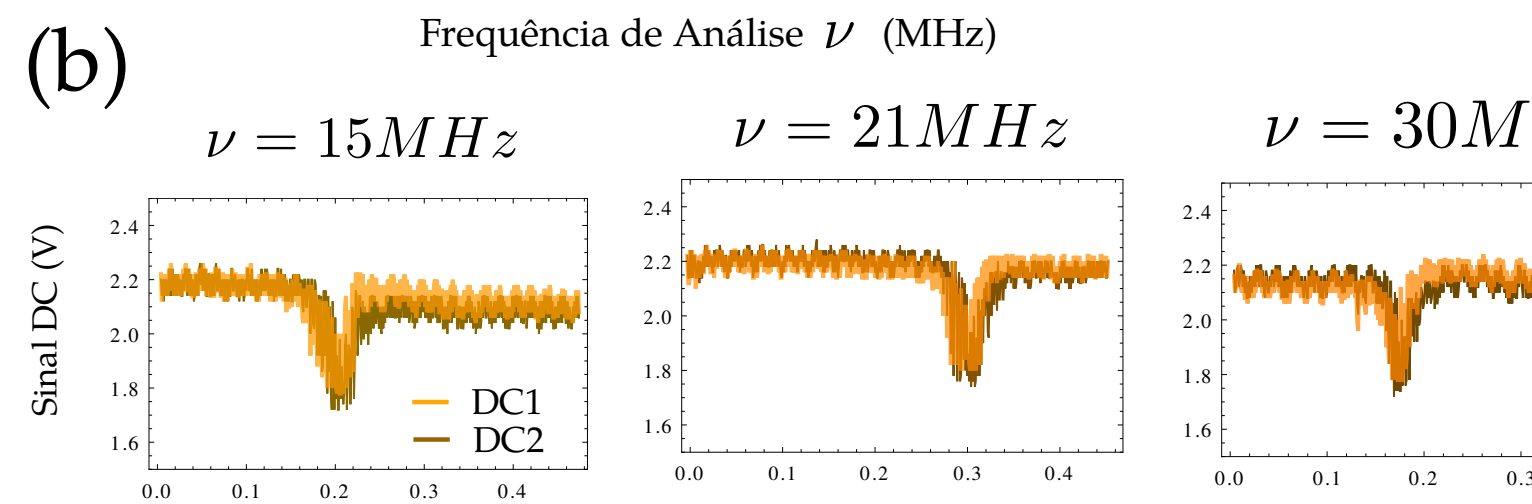

$\nu=30 M H z$
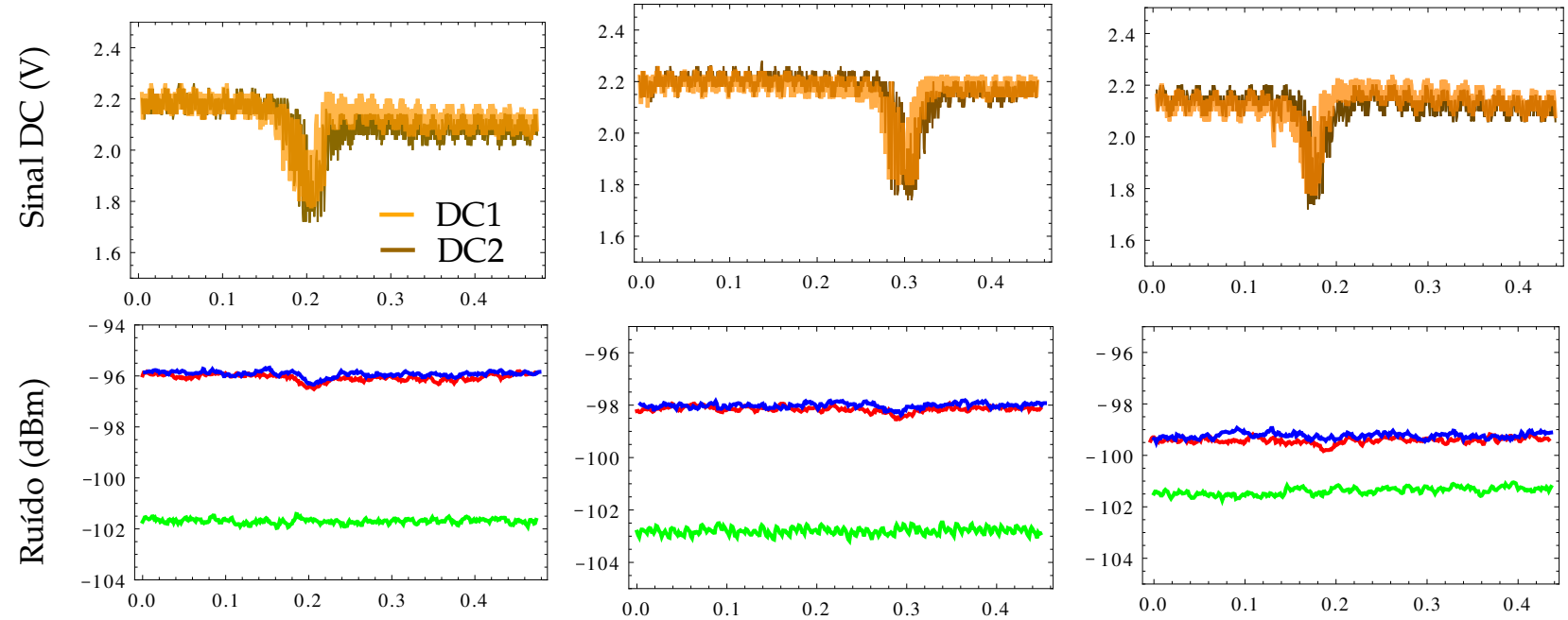

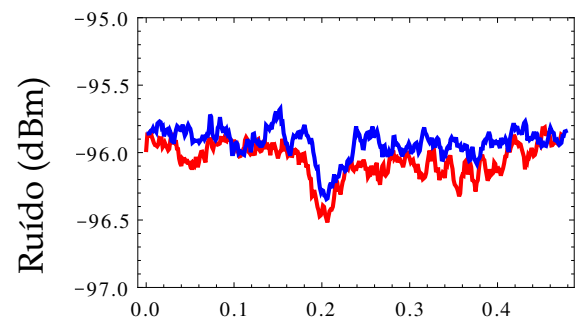

Tempo de Mod. (s)

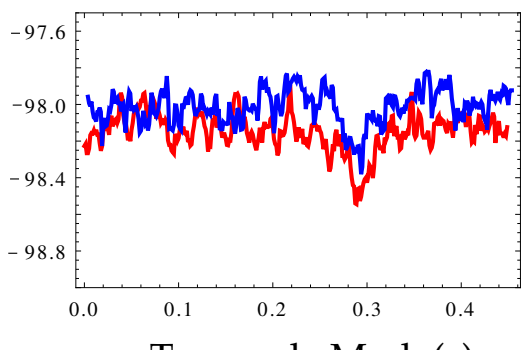

Tempo de Mod. (s)
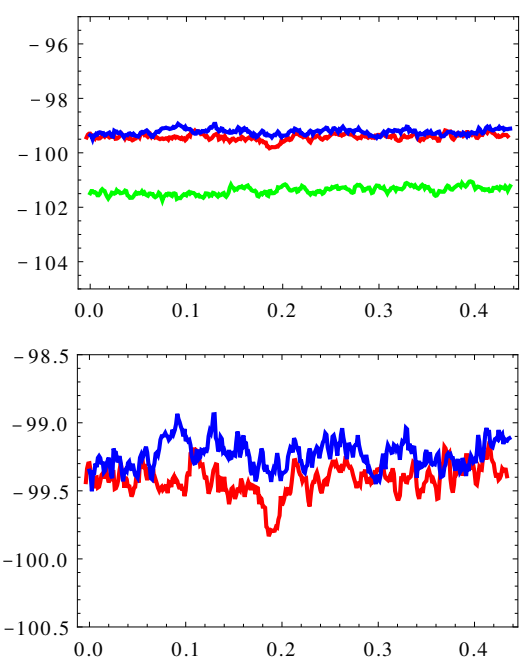

Tempo de Mod. (s)

Figura 4.8: (a) Espectro de ruído do laser de titânio safira para uma potência $P=4.501(2) \mathrm{mW}$. (b) Rotação de elipse para as frequências de análise $\nu=15 \mathrm{MHz}, 21 \mathrm{MHz}$ e $30 \mathrm{MHz}$.

campo magnético de aprisionamento e o feixe de rebombeio da MOT durante 4 ms. Após desligar o campo magnético esperamos $3 \mathrm{~ms}$ para reduzir a presença de campo magnético 
em até $99 \%$ na região da nuvem. Depois dos 3 ms, acoplamos um bombeio ótico na transição $F=2 \rightarrow F^{\prime}=2$ que transfere parte da população resfriada no estado $F=2$, para o estado $F=1$, onde a espectroscopia de EIT será feita. A varredura de frequência num dos feixes é feita ao mesmo tempo que o bombeio ótico é ligado. A varredura de frequência é feita tipicamente em 0.5 ms, durante o qual fazemos aquisição do sinal DC e sinal HF dos detectores. Finalmente ligamos o campo magnético de aprisionamento e os feixes de resfriamento novamente para carregar a nuvem de átomos durante $1 \mathrm{~s}$. Repetimos este ciclo cem vezes para obter uma média de dados com os quais determinamos a correlação das intensidades.

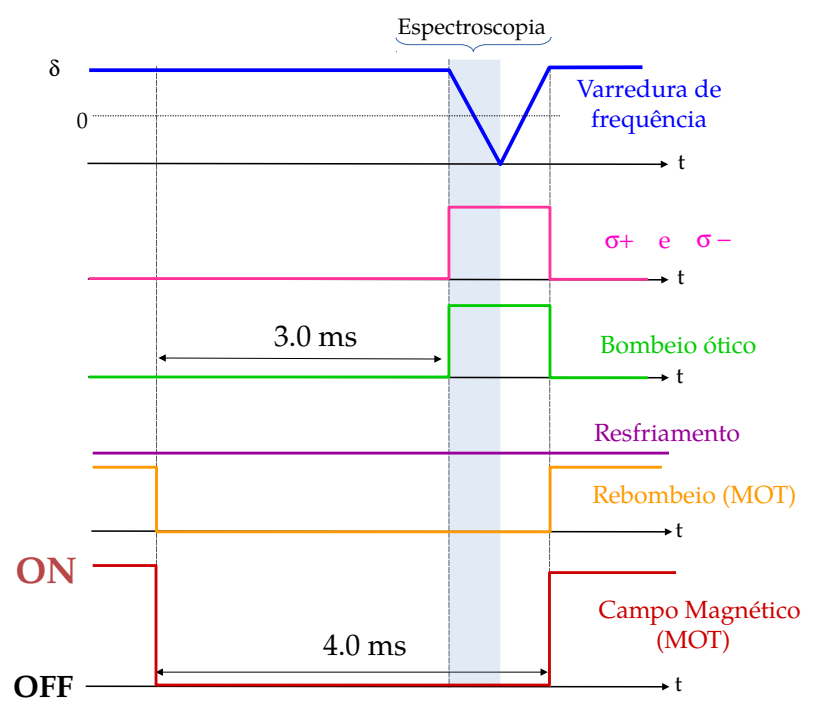

Figura 4.9: Sequência temporal para realizar a espectroscopia dos átomos em condição de EIT

\subsection{Aplicando dessintonia de um fóton na espectroscopia de correlação}

Até o ano 2012, os espectros de correlação sempre foram feitos deixando um dos campos em ressonância $\Delta_{1}=0$ e varrendo a frequência do outro feixe $\Delta_{2}$ de forma linear no tempo (ver 4.2.a). Durante o primeiro semestre de 2013 comecei o estudo da espectroscopia de correlação variando a dessintonia de um fóton $\Delta_{1} \neq 0$, que analisaremos a seguir. Ao longo desse tempo chegaram mais dois membros da equipe, a Klara (estudante de doutorado) e o 
Ashok (pós-doutorando), conseguindo realizar um estudo completo e uma análise eficiente dos dados sobre várias propriedades da correlação que serão tratadas nestas duas próximas seções.

Começaremos analisando os resultados para 3 casos principais de dessintonia de um fóton $\Delta_{1} / 2 \pi$ (ver figura 4.2.(a)):-6MHz (a), $0 \mathrm{MHz}$ (b) e $5 \mathrm{MHz}(\mathrm{c})$. Cada fileira na figura 4.10 representa diferentes características da espectroscopia. Na primeira fileira mostramos o sinal DC, que mede a transmissão dos feixes e está associada ao valor médio da fotocorrente. Na segunda fileira mostramos a função de correlação $C(\omega)$ para uma frequência de análise $\omega / 2 \pi=2 \mathrm{MHz}$. E na terceira fileira mostramos o cálculo dos elementos de matriz $\tilde{\Pi}_{i j}$ multiplicados pelo seus coeficientes associados $\nu(\omega)_{i j}$, que determinam os espectros de ruído (3.116). Com eles poderemos realizar um mapeamento da correlação medida experimentalmente com alguns parâmetros do modelo perturbativo descrito na seção 3.6.2. Todas as curvas são traçadas em função da dessintonia de dois fótons $\delta=\Delta_{2}-\Delta_{1}$. Em todas elas, as linhas verticais tracejadas mostram a janela de transparência $\Delta \omega_{E I T}$ obtida a partir do sinal DC. Os espectros foram obtidos mantendo uma potência de 112(1) $\mu \mathrm{W}$ (cintura ${ }^{2}$ de $1.8(4) \mathrm{mm}$ ) e $117(1) \mu \mathrm{W}$ (cintura 1.5(4)mm) para os feixes 1 e 2, respectivamente. A partir dos parâmetros experimentais, determinamos os seus equivalentes teóricos para calcular as correlações $C(\omega)$ e $g^{(2)}(0)$. Esses parâmetros serão especificados em cada figura. A taxa de decoerência $\gamma_{d}$ é o único parâmetro livre dentro dos cálculos.

Consideremos o caso ressonante $\Delta_{1}=0$ da figura $4.10(\mathrm{~b})$. Neste caso, podemos observar o perfil típico de EIT no sinal DC, com o qual podemos determinar a largura $\Delta \omega_{E I T} \sim 2$ $\mathrm{MHz}$ a partir da largura a meia altura do pico de EIT. Dentro deste perfil podemos notar que a correlação apresenta o mesmo perfil registrado em [44] e analisado em detalhe no capítulo 3. Para $|\delta| / 2 \pi>5 \mathrm{MHz}$, observamos que as intensidades dos campos estão anticorrelacionadas, assim como para as dessintonias $|\delta| / 2 \pi<2 \mathrm{MHz}$. Entretanto, na ressonância $\delta=0$, a correlação se caracteriza pela largura intrínseca que é menor do que $\Delta \omega_{E I T}$, e que é livre de alargamento por potência como foi discutido nas seções 3.4 e 3.5.4. Neste caso, a largura

\footnotetext{
${ }^{2}$ Consideramos a cintura definida para um feixe gaussiano quando a intensidade diminuiu para um fator de $1 / e^{2}$.
} 


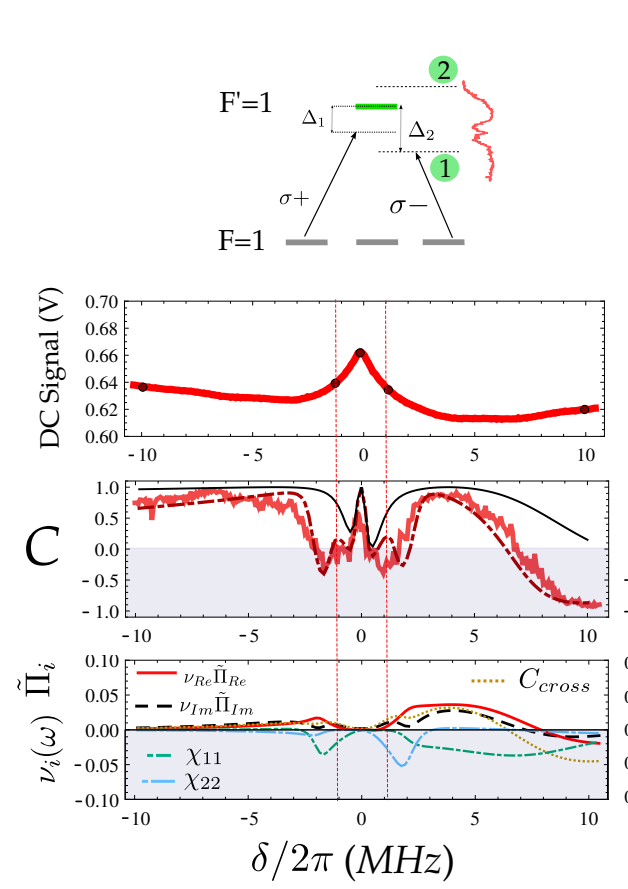

(a)
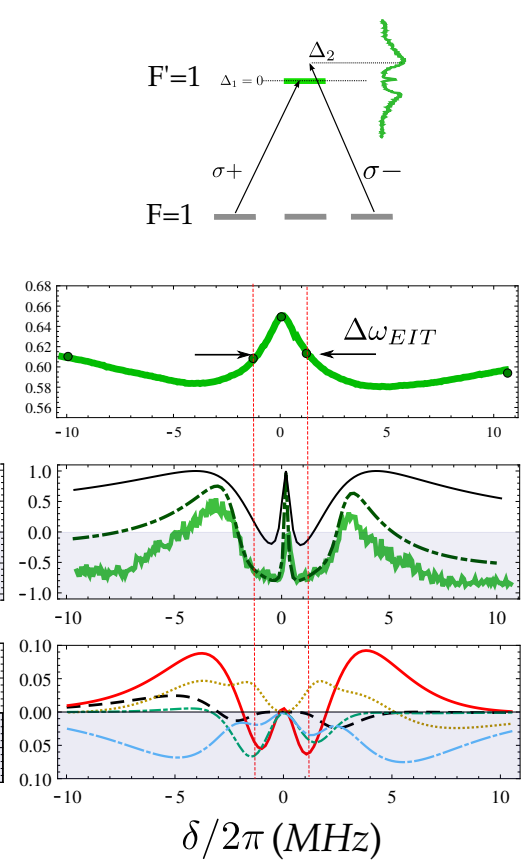

(b)
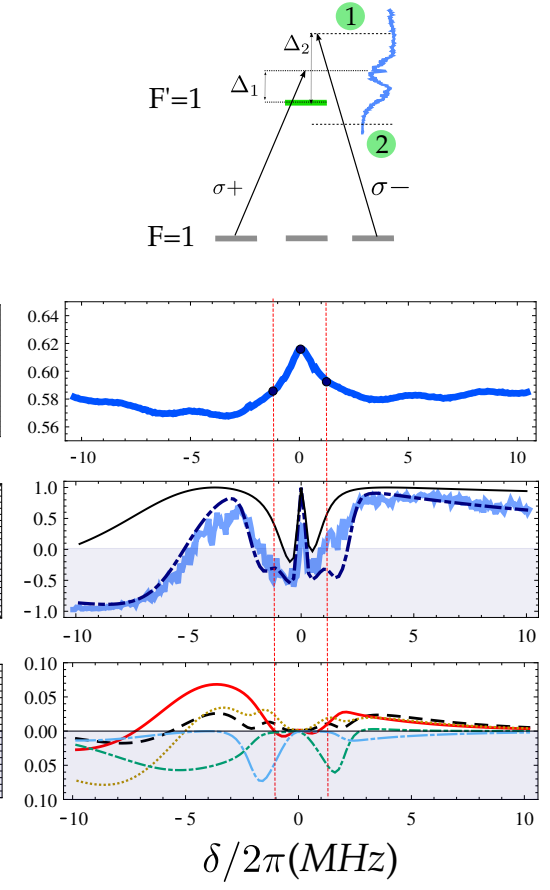

(c)

Figura 4.10: Resultados da espectroscopia para três diferentes parâmetros: sinal DC (primeira fileira), correlação (segunda fileira) e decomposição em termos dos produtos $\nu(\omega)_{i} \tilde{\Pi}_{i}$ da eq.(3.120) do tratamento perturbativo (terceira fileira). Espectroscopia para dessintonia de um fóton $\Delta_{1} / 2 \pi=$ $-6 \mathrm{MHz}(a), \Delta_{1}=0 \mathrm{MHz}(\mathrm{b})$ e $\Delta_{1} / 2 \pi=5 \mathrm{MHz}$ (c). O espectro de correlação experimental é traçado em linha grossa sólida. Os resultados para o tratamento perturbativo de $C(\omega)$ e o modelo heurístico para $g^{(2)}(0)$ são traçados pelas linhas pontilhada-tracejada e pela linha fina sólida, respectivamente (na segunda fileira). Os parâmetros empregados para o meio atômico são: taxa de decoerência $\gamma_{d} / 2 \pi=150 \mathrm{kHz}$, frequência de análise $\omega / 2 \pi=2 \mathrm{MHz}$, ruído de fase $\bar{\gamma} / 2 \pi=1 \mathrm{MHz}$. As frequências de Rabi associadas aos valores experimentais de intensidade são $\Omega_{1}=0.30 \Gamma$ and $\Omega_{2}=0.34 \Gamma$. As dessintonias de um fóton para os espectros teóricos são $\Delta_{1} / 2 \pi=-6 \mathrm{MHz}(a), 0.2 \mathrm{MHz}(\mathrm{b})$ and $5 \mathrm{MHz}$ (c), respectivamente.

intrínseca é 207(9) kHz. A função de correlação $g^{(2)}(0)$ do modelo heurístico da eq.(3.35) e a correlação $C(\omega)$ do modelo perturbativo da eq.(3.120), também foram traçadas em linha solida e linha pontilhada-tracejada, respectivamente. Embora a correlação $g^{(2)}(0)$ apresenta um perfil similar ao da correlação $C(\omega)$ (experimental e teórica), o resultado heurístico mostra que os campos estão dominados pela correlação $\left(g^{(2)}(0)>0\right)$, com uma tênue anti-correlação em $|\delta| / 2 \pi \sim 1 \mathrm{MHz}$. Isto é consequência do fato de considerar unicamente a combinação de $\operatorname{Im} p_{1} \operatorname{Im} p_{1}$ e $\operatorname{Re} p_{1} \operatorname{Re} p_{1}$ na correlação da eq. (3.35) [43], em que para a maioria das dessintonias 
o termo de absorção (positivo) domina sobre o termo de dispersão (negativo). Este é um dos casos analisados na figura 3.11.

Entretanto, a correlação $C(\omega)$ é calculada a partir do tratamento perturbativo, o qual descreve de forma satisfatória a correlação experimental. Para simplificar a análise, adotamos uma notação para cada um dos termos $\nu_{i j}(\omega) \tilde{\Pi}_{i j}$ da correlação (3.120) mais alusiva às suas propriedades. Portanto reescrevemos a correlação da eq.(3.120) como

$$
C(\omega)=\frac{\nu_{I m}(\omega) \tilde{\Pi}_{I m}+\nu_{R e}(\omega) \tilde{\Pi}_{R e}+C_{c r o s s}(\omega)+C_{1 \mid 2}(\omega)}{\sqrt{S_{11}(\omega) S_{22}(\omega)}},
$$

onde o produto dispersivo e de absorção são redefinidos como $\nu_{R e}(\omega) \tilde{\Pi}_{R e} \equiv \nu_{46}(\omega) \tilde{\Pi}_{46}$ e $\nu_{I m}(\omega) \tilde{\Pi}_{I m} \equiv \nu_{35}(\omega) \tilde{\Pi}_{35}$, respectivamente. Além disso, unificamos os termos cruzados em um único termo $C_{C r o s s}(\omega)=\nu_{R I}(\omega) \tilde{\Pi}_{R I}(\omega)+\nu_{I R}(\omega) \tilde{\Pi}_{I R}(\omega) \equiv \nu_{36}(\omega) \tilde{\Pi}_{36}+\nu_{45}(\omega) \tilde{\Pi}_{45}$ e os termos restantes em $\chi_{11}(\omega) \equiv \nu_{44}(\omega) \tilde{\Pi}_{44}+\nu_{33}(\omega) \tilde{\Pi}_{33}+\nu_{34}(\omega) \tilde{\Pi}_{34}$ e $\chi_{22}(\omega) \equiv \nu_{66}(\omega) \tilde{\Pi}_{66}+$ $\nu_{55}(\omega) \tilde{\Pi}_{55}+\nu_{56}(\omega) \tilde{\Pi}_{56}$, onde $\chi_{11}(\omega)+\chi_{22}(\omega)=C_{1 \mid 2}(\omega)$. Tendo feito essa ressalva na notação adotada, podemos estender a análise feita na seção 3.6.2, onde discutimos o mapeamento da correlação com os produtos $\nu_{i j}(\omega) \tilde{\Pi}_{i j}$, ou seja, como a dispersão $\left(\nu_{R e}(\omega) \tilde{\Pi}_{R e}\right)$ domina sobre a absorção $\left(\nu_{I m}(\omega) \tilde{\Pi}_{I m}\right)$ para $|\delta|<3 \Delta \omega_{E I T}$, como o termo cruzado $C_{C r o s s}$ determina a inversão no sinal da correlação para $|\delta|>3 \Delta \omega_{E I T}$, etc.

Agora analisaremos os dois casos em que os campos são dessintonizados da ressonância atômica: $\Delta_{1}=-6 \mathrm{MHz}$ e $\Delta_{1}=5 \mathrm{MHz}$, como mostram as figuras 4.10.(a) e (c), respectivamente. Podemos começar observando que $C(\omega)$ apresenta um aumento no nível de correlação em relação ao caso em que $\Delta_{1}=0$, no intervalo de dessintonias $|\delta|<4 \mathrm{MHz}$. Entretanto, para uma dessintonia $|\delta|>5 \mathrm{MHz}$, ambos os espectros mostram uma assimetria no nível de correlação em torno da ressonância de dois fótons $\delta=0$. Por exemplo, no caso de dessintonia $\Delta_{1}=5 \mathrm{MHz}$ da figura 4.10.(c), o espectro apresenta uma saturação de correlação para $\delta>5 \mathrm{MHz}$, enquanto que o coeficiente $C$ toma valores negativos para $\delta<-5 \mathrm{MHz}$ indicando anticorrelação. O espectro de correlação para $\Delta_{1}=-6 \mathrm{MHz}$ da figura 4.10.(a) é um espectro quase espelhado ao caso de $\Delta_{1}=5 \mathrm{MHz}$ da figura 4.10.(c).

A partir do que acabamos de descrever, podemos constatar uma característica dos espec- 
tros dessintonizados. A mudança de correlação para anti-correlação ocorre exatamente na ressonância do campo $\mathbf{E}_{2}\left(\Delta_{2}=0 \mathrm{MHz}\right.$ que corresponde a $\left.\delta=-\Delta_{1}\right)$. Pelo modelo perturbativo, este comportamento é seguido de forma muito próxima pelo termo cruzado $C_{\text {cross }}$, enquanto que o termo dispersivo $\tilde{\Pi}_{R e}$, junto com o termo de absorção $\tilde{\Pi}_{I m}$, são compensados pelos termos $\chi_{11}(\omega)$ e $\chi_{22}(\omega)$. Descrever a correlação unicamente pelos $\tilde{\Pi}_{I m}$ e $\tilde{\Pi}_{R e}$, os quais determinam a correlação $g^{(2)}(0)$, não justifica a anticorrelação observada no domínio das frequências. A função $g^{(2)}(0)$, no entanto, se apresenta como uma função evolvente a $C(\omega)$.

Este ponto de transição correlação-anticorrelação $\left(\delta=-\Delta_{1}\right)$ poder ser interpretado da seguinte maneira: as flutuações de frequência (que equivalem à difusão de fase) são comuns para ambos os campos, pois eles são gerados pelo mesmo laser. Portanto, essas flutuações são convertidas em flutuações de intensidade (mecanismo RF-RA discutido na seção 3.3), pela variação da taxa de absorção dos campos à medida que as frequências dos campos se aproximam ou afastam da ressonância atômica. Assim, quando as dessintonias de ambos os campos $\left(\Delta_{1}\right.$ e $\left.\Delta_{2}\right)$ possuem o mesmo sinal (situação 1 na parte superior da figura 4.10), as flutuações de intensidade serão correlacionadas. Por outro lado, se aplicamos dessintonias no campos com sinal oposto (situação 2), resultará numa anti-correlação das flutuações de intensidade.

No que diz respeito à correlação para $|\delta|<5 \mathrm{MHz}$, notamos que ela é pouco resolvida nos espectros das figuras 4.10.(a) e (c). Portanto, fizemos espectroscopias com maior resolução para estudar a correlação na proximidade da ressonância de dois fótons. A figura 4.11 mostra uma sequência de espectros com intervalos de varredura espectral $|\delta|<4 \mathrm{MHz}$. A figura 4.11.(a), mostra em linha de cor vermelha, os espectros para as dessintonias negativas $\Delta_{1}=-1,-2,-4$ e $-5 \mathrm{MHz}$, e a figura (b) mostra, em linha de cor azul, os espectros para a dessintonias positivas $\Delta_{1}=6,4,3$ e $1 \mathrm{MHz}$. Cada um dos espectros está acompanhado pela sua respectiva curva teórica dada pelo modelo perturbativo.

A sequência de espectros na figura 4.11.(a), mostra uma transição de anticorrelação para correlação na região $\Delta \omega_{\text {Int }}<|\delta|<\Delta \omega_{E I T}$, à medida que aumentamos a dessintonia negativa de um fóton. No caso de maior dessintonia negativa $\left(\Delta_{1}=-5 \mathrm{MHz}\right)$, a correlação apresenta 
uma resposta particular em torno da dessintonia $\delta / 2 \pi \sim-\omega_{a} / 2 \pi=2 \mathrm{MHz}$, que corresponde justamente à frequência de análise da demodulação do sinal HF das fotocorrentes nos detectores. Esta estrutura também é descrita pelo espectro obtido teoricamente. Para o caso de dessintonia positivas $\left(\Delta_{1}>0\right)$ da figura (b), o comportamento da correlação é similar, mas espectralmente espelhado. Para $\Delta_{1}=6 \mathrm{MHz}$ também observamos uma certa ressonância para $\delta / 2 \pi \sim \omega_{a}=2 \mathrm{MHz}$.

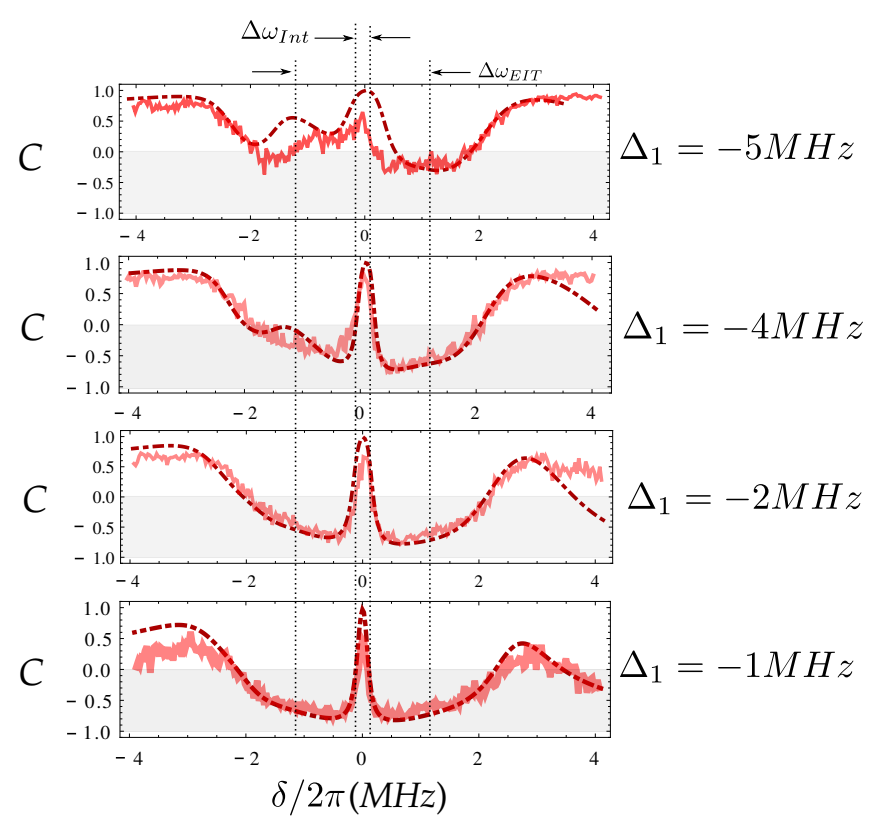

(a)

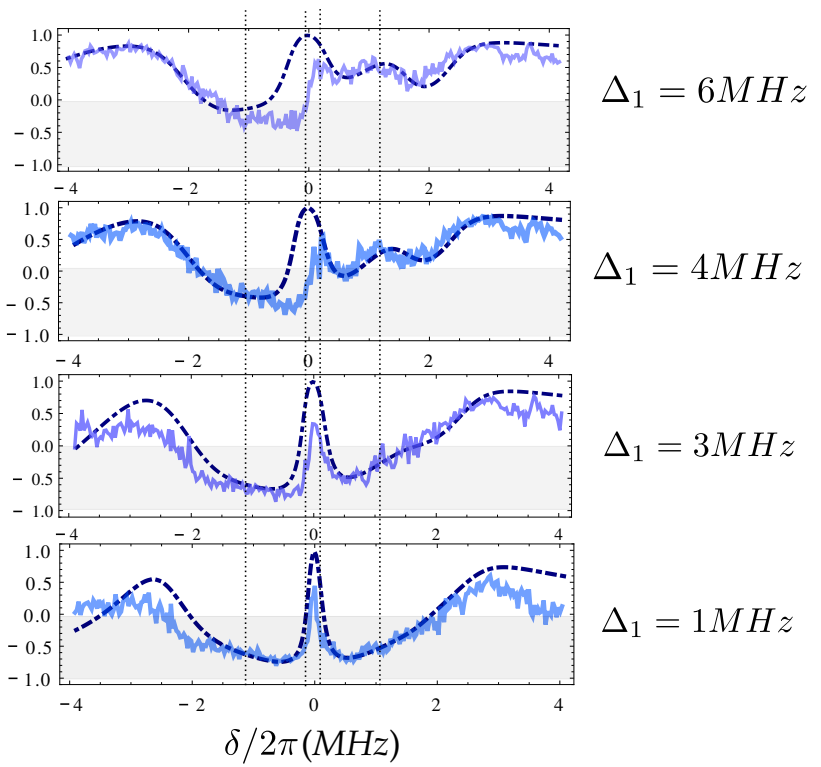

(b)

Figura 4.11: Espectro de correlação teórico e experimental em função da dessintonia de dois fótons $\delta$, para: (a) dessintonias positivas $\Delta_{1}=6,4.2,3,1 \mathrm{MHz}$, e (b) dessintonias negativas $\Delta_{1}=-1.0,-2$, $-4,-5 \mathrm{MHz}$.

\subsubsection{Ressonâncias $\delta= \pm \omega_{a}$ na espectroscopia de correlação}

Para obter uma melhor compreensão do papel das bandas laterais na correlação dos campos, analisaremos os espectros para diferentes frequências de analise $\omega_{a}$, no caso ressonante nas figuras $4.12(\mathrm{a}-\mathrm{c})$, e também para o caso dessintonizado $\Delta_{1} / 2 \pi=6 \mathrm{MHz}$ nas figuras 4.12(d-f). Na primeira fileira (figuras $4.12(\mathrm{a}, \mathrm{d})$ ), mostramos os espectros obtidos para $\omega_{a} / 2 \pi=2 \mathrm{MHz}$ discutidos anteriormente. Na segunda (figuras $4.12(\mathrm{~b}, \mathrm{e})$ ) e na terceira fileira 
(figuras. 4.12(c,f)), mostramos os espectros para $\omega_{a} / 2 \pi=3 \mathrm{MHz}$ e $4 \mathrm{MHz}$, respectivamente.
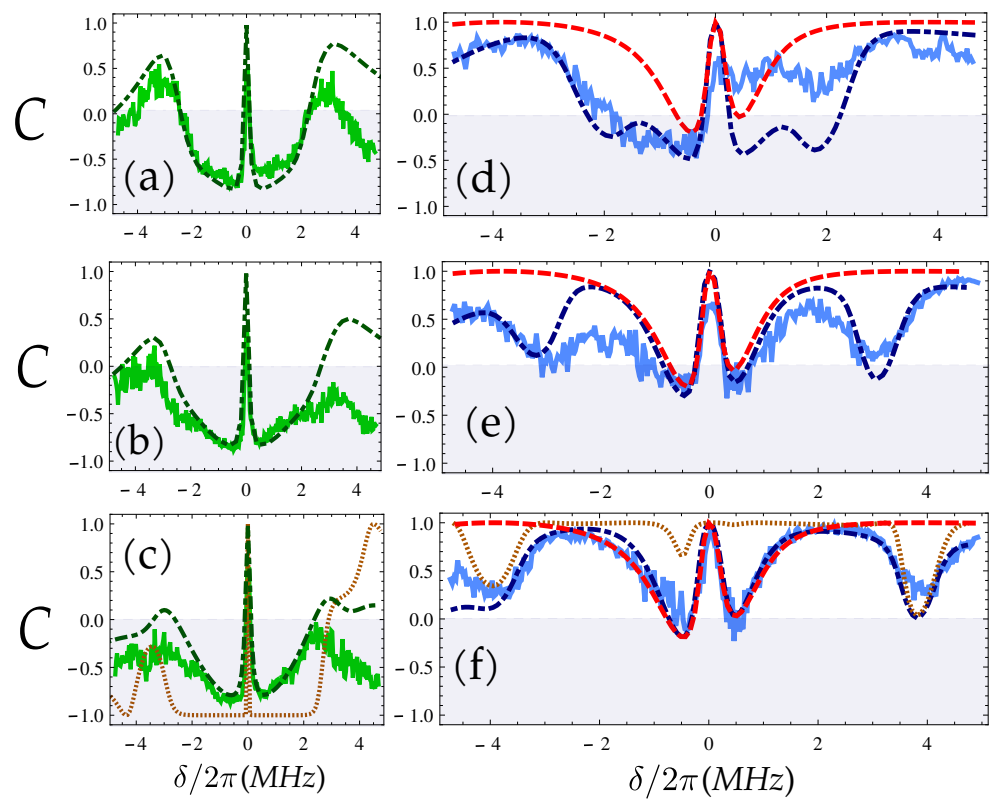

Figura 4.12: Espectroscopia de correlação para diferentes frequências de análise $\left(\omega_{a} / 2 \pi\right)$ com $\Delta_{1}=$ $0 \mathrm{MHz}$ (primeira coluna) e $\Delta_{1} / 2 \pi=6 \mathrm{MHz}$ (segunda coluna). (a, d) $\omega_{a} / 2 \pi=2 \mathrm{MHz},(b, e) \omega_{a} / 2 \pi=$ $3 \mathrm{MHz}$ e $(c, f) \omega_{a} / 2 \pi=4 \mathrm{MHz}$. A correlação experimental $C(\omega)$ é traçada em linha sólida, o resultado teórico para $C(\omega)$ é traçado em linha pontilhada-tracejada e $g^{(2)}(0)$ é traçada em linha tracejada. Nas figuras (c, $f$ ), é traçado (em linha pontilhada) o termo de primeira ordem do tratamento perturbativo.

O resultado perturbativo para $C(\omega)$ também é traçado (linha pontilhada-tracejada) junto com os dados experimentais (linha grossa sólida). $\mathrm{O}$ termo à primeira ordem na correlação $\epsilon^{2}$ é traçado em linha pontilhada para $\omega_{a} / 2 \pi=4 \mathrm{MHz}$. No caso ressonante $\Delta_{1}=0$ (ver figuras $4.12(\mathrm{a}-\mathrm{c}))$, para $\omega_{a} / 2 \pi=2,3$ e $4 \mathrm{MHz}$, não há uma independência espectral das bandas laterais no espectro da correlação para $C(\omega)$, como foi discutido na seção 3.20. A largura intrínseca da correlação é $147(9) \mathrm{kHz}$, de acordo com a largura do pico central, e é consistente com a taxa de decoerência empregada para realizar o cálculo teórico $(150 \mathrm{kHz})$. Embora a ressonância na frequência da banda lateral $\delta \sim-\omega_{a}$ pareça ser resolvida à primeira ordem pelo modelo perturbativo (traçado em linha pontilhada na figura 4.12.(c)), a contribuição de termos de ordem superior suprime tais ressonâncias. Isto se deve ao fato dos campos, ao estarem próximos da ressonância atômica (dentro da largura natural $\sim 6 \mathrm{MHz}$ ), a conversão de ruído RF-RA é dominada pela emissão espontânea e estimulada, populando as bandas 
laterais de tal forma que as ressonâncias na frequência de análise não são resolvidas.

Agora, consideremos o caso não ressonante $\left(\Delta_{1} / 2 \pi=6 \mathrm{MHz}\right)$ apresentado nas figuras 4.12(d-f). Podemos notar que as ressonâncias nas frequências das bandas laterias $\delta= \pm \omega_{a}$ são espectralmente resolvidas se $\omega_{a}>\Delta \omega_{E I T}$ é satisfeito. Para $|\delta|<\Delta \omega_{E I T}$, os campos intensos (cuja energia se concentra na frequência portadora) determinam o perfil de correlação (figuras 4.12(e,f)). Assim, a função $g^{(2)}(0)$ se superpõe à correlação $C(\omega)$ para $|\delta|<\Delta \omega_{E I T}$, onde as portadoras estão próximas da ressonância de dois fótons. Cabe notar que a largura intrínseca para $C(\omega)$ e $g^{2}(0)$ é a mesma $(200 \mathrm{kHz})$. Entretanto, na estrutura do intervalo de dessintonias $|\delta|>\Delta \omega_{E I T}$, a redução de correlação para $\delta= \pm \omega_{a}$ se torna mais evidente conforme a frequência de análise aumenta. A figura 4.12(f) mostra que tal redução na correlação já está presente na primeira ordem de perturbação. Em outras palavras, dado que a resposta atômica à frequências das bandas laterias depende unicamente dos coeficientes $\nu(\omega)$, a correlação à primeira ordem parece não ser afetada pelas correções perturbativas nas ressonâncias das bandas laterais. Isto não acontece com o caso ressonante da figura 4.12(c).

Em seguida analisaremos a correlação para o caso de $\omega_{a} / 2 \pi=4 \mathrm{MHz}$ mediante a decomposição do modelo perturbativo. Na parte superior da figura 4.13 colocamos o mesmo espectro de correlação da figura 4.12(f). A figura 4.13(a) mostra os principais elementos $\tilde{\Pi}_{i j}$, e as figuras (b), (c) e (d) mostram os coeficientes $\nu(\omega)_{i j}$ que dão peso aos elementos $\tilde{\Pi}_{i j}$. As figuras 4.13(c) e (d) mostram os coeficientes $\nu(\omega)_{i j}$, que definem os termos $\chi_{11}(\omega)$ e $\chi_{22}(\omega)$, respectivamente, das eqs.(A.7-A.8). Ao contrário do caso ressonante da figura 4.10(b) e 4.12(c), onde $\nu_{I m} \ll \nu_{R e}$, no caso não ressonante $\nu_{I m} \sim \nu_{R e}$ para $|\delta| / 2 \pi<3 \mathrm{MHz}$ (ver figura 4.13(a)). Portanto, a resposta de absorção e dispersão contribuem igualmente para a correlação de intensidade, enquanto que o termo cruzado $C_{\text {cross }}$ é aproximadamente cancelado devido ao comportamento oposto do elementos $\tilde{\Pi}_{R I}$ e $\tilde{\Pi}_{I R}$. A parte residual deste termo cruzado é cancelado pelo termo $\nu_{56}(\omega)$, como mostra a figura (d). O resto dos coeficientes $\nu_{i j}(\omega)$ para os termos extra $\chi_{11}(\omega)$ e $\chi_{22}(\omega)$, quase não contribuem na correlação para $|\delta| / 2 \pi<3 \mathrm{MHz}$, como mostram as figuras 4.13(c) e (d). Portanto, as duas principais contribuições correspondem às respostas de absorção e dispersão, o que coincide com o modelo heurístico onde 
os dois termos se caracterizam por terem a mesma contribuição para a função $g^{(2)}(0)$ da eq.(3.35). É por esta razão que as duas funções se superpõem e descrevem a mesma correlação de intensidade para $|\delta| / 2 \pi<3 \mathbf{M H z}$ no caso não ressonante, onde a frequência de análise satisfaz $\omega>\Delta \omega_{E I T}$.

Este resultado surpreende pela clara superposição entre as duas funções, pois ao longo da seção 3.6.2 e na primeira parte desta seção, nos concentramos em ressaltar a constante diferença que havia entre as funções $g^{(2)}(0)$ e $C(\omega)$. No entanto, esta superposição deixa claro que há então condições de dessintonia para as quais o modelo heurístico é compatível com o modelo perturbativo, e ambos expõem nitidamente que as únicas variáveis associadas ao perfil de correlação são a dispersão e a absorção. Isto indica que eventuais análises dos espectros de correlação para qualquer aplicação no futuro em sistemas $\Lambda$ (medida da largura intrínseca, entre outros), podem simplesmente ser aplicados ao modelo heurístico sem falta de precisão na descrição do sistema, sempre e quando se satisfaça a condição $\Delta_{1}>\Gamma$.

No que diz respeito às ressonâncias nas bandas laterais, todos os coeficientes $\nu(\omega)_{i j}$ apresentam ressonâncias exatamente nas frequências de análise $\delta \sim \pm \omega_{a}= \pm(2 \pi) 4 \mathrm{MHz}$. Os coeficientes principais $\nu_{I m}(\omega)$ e $\nu_{R e}(\omega)$ mostram comportamentos opostos próximos à frequência de análise (figura 4.13 (a)). O coeficiente $\nu_{R e}$ em linha sólida mostra um incremento na resposta dispersiva, enquanto que $\nu_{I m}(\omega)$ diminui. No entanto, para as ressonâncias nas bandas laterais, a contribuição dos termos extra associados a $\chi_{11}(\omega)$ e $\chi_{22}(\omega)$ nas figuras 4.13(c) e (d) também competem, induzindo uma correlação $C(\omega) \sim 0$. Cabe notar que as ressonâncias nas bandas laterais são largas, com quase a metade da largura $\Delta \omega_{E I T}$. Como veremos a seguir, elas são sensíveis a alargamento por potência.

\subsubsection{Alargamento por potência das ressonâncias nas bandas laterais}

Nesta seção investigaremos brevemente como é o espectro de correlação para diferentes intensidades dos campos, no caso não ressonante. A figura 4.14 (a) mostra o espectro de correlação para duas potências diferentes dos campos. Para uma potência de $110 \mu \mathrm{W}$ em cada campo, temos uma intensidade $I / I_{\text {sat }}=0.23$ (linha sólida), em que as ressonâncias 


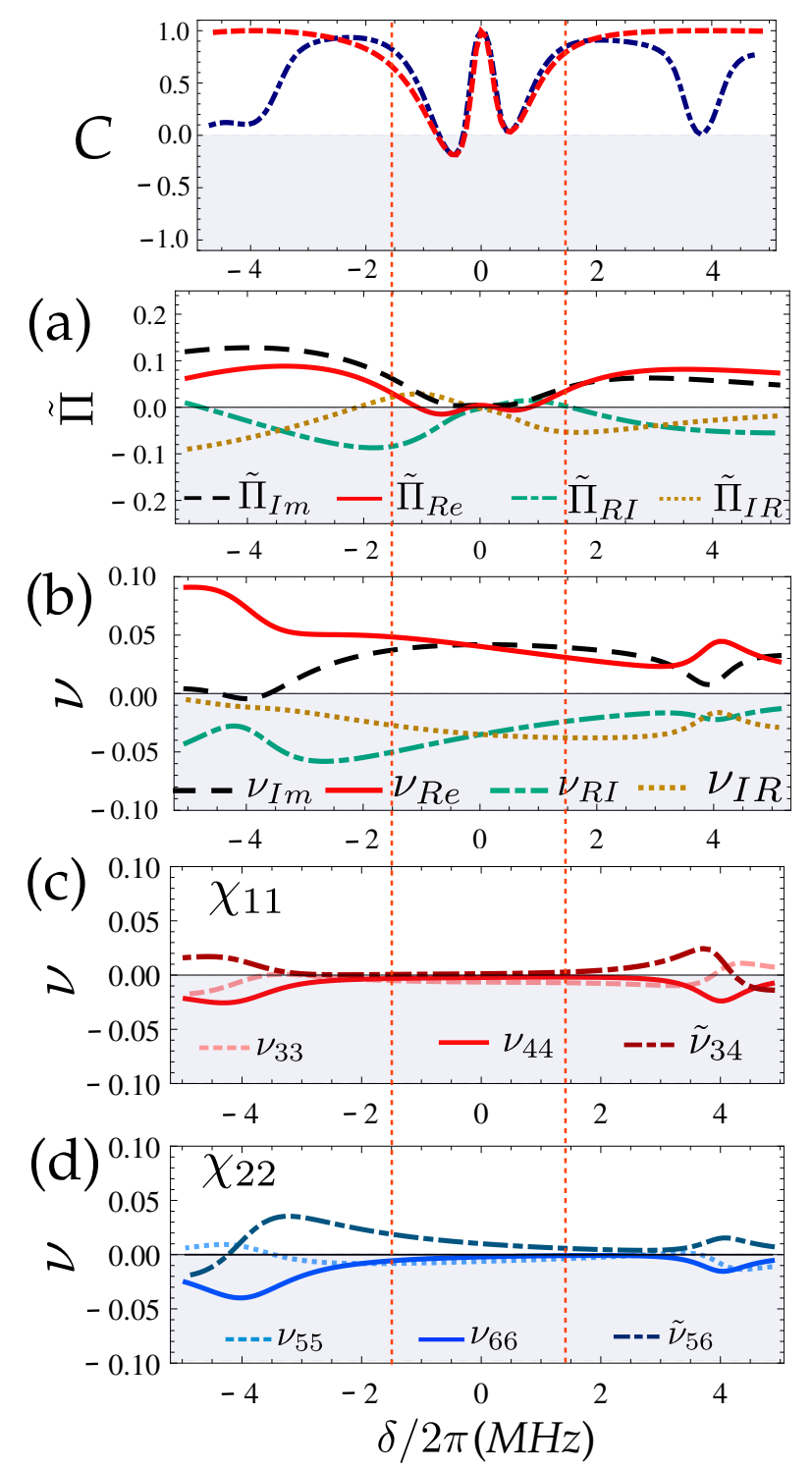

Figura 4.13: Mapeamento entre a intensidade de correlação $C(\omega)$ da figura 4.12.(f) e: (a) principais elementos $\tilde{\Pi}_{i j}$, (b) coeficientes $\nu_{I m}(\omega), \nu_{I m}(\omega), \nu_{I R}(\omega)$ e $\nu_{R I}(\omega)$ associados aos produtos conjuntos. Nas figuras $(c)$ e (d) traçamos os coeficientes $\nu(\omega)_{i j}$ associados aos termos extra $\chi_{11}(\omega)$ e $\chi_{22}(\omega)$ definidos na eq.(A.6). Esta análise é feita para $\Delta_{1} / 2 \pi=5 \mathrm{MHz}$ e $\omega_{a} / 2 \pi=4 \mathrm{MHz}$. As duas linhas pontilhadas representam a janela de transparência $\Delta \omega_{E I T}$.

$\delta= \pm \omega_{a}$ são resolvidas. No entanto, ao aumentar a potência para $190 \mu \mathrm{W}$ (linha pontilhada), que corresponde a uma intensidade $I / I_{\text {sat }}=0.40$, a ressonância para $\delta=-\omega_{a}$, próxima da ressonância atômica, é sensível a alargamento por potência.

Se aumentarmos as potências dos campos isto implicaria no aumento significativo das 


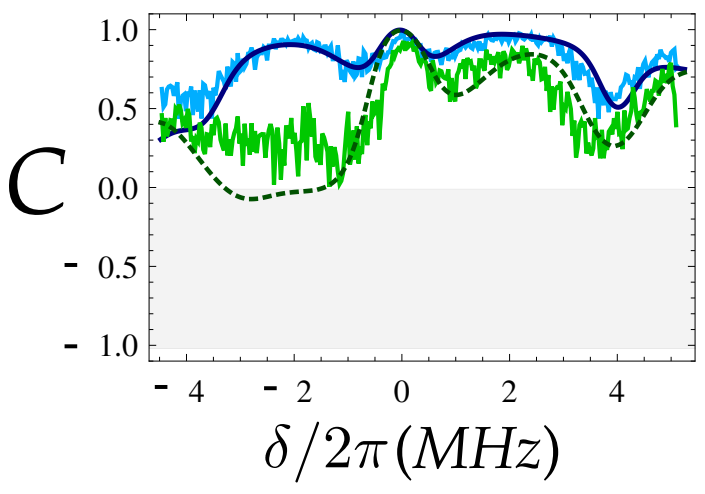

(a)

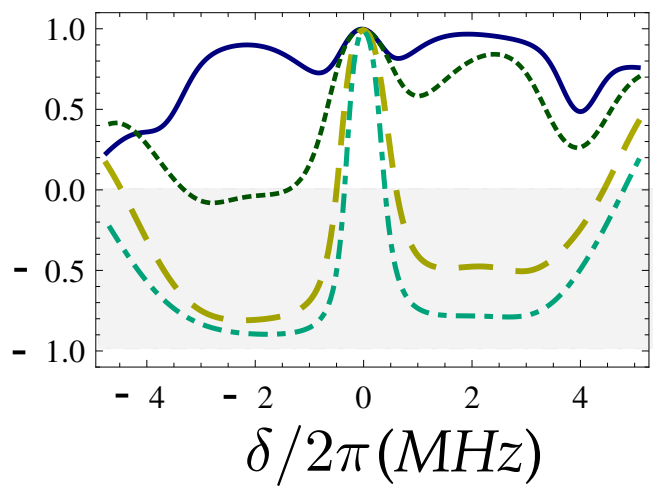

(b)

Figura 4.14: Espectro de correlação para diferentes potências no feixes, para o caso não ressonante $\Delta_{1} / 2 \pi=5 \mathrm{MHz}$ e uma frequência de análise de $4 \mathrm{MHz}$. (a) Espectros de correlação experimentais $110 \mu \mathrm{W}$ (linha sólida) e 190 $\mu \mathrm{W}$ (linha pontilhada) com suas respectivas curvas teóricas. (b) Resultados teóricos para o espectro de correlação (traçados em linha sólida) para as mesmas potências usadas na figura (a), e para mais duas potências, $3 \times 110 \mu \mathrm{W}$ (linha tracejada) e $4 \times 110 \mu \mathrm{W}$ (linha pontilhadatracejada).

forças de espalhamento que a luz exerce nos átomos durante a toma de dados nesse intervalo de tempo. No entanto, podemos analisar o caso para potências maiores mediante o modelo teórico. A figura 4.14 (b) mostra os resultados teóricos da correlação para os dois casos de potência da figura 4.14 (a), e também para dois casos de potências maiores, $3 \times 110 \mu \mathrm{W}$ (linha tracejada) e $4 \times 110 \mu \mathrm{W}$ (linha pontilhada-tracejada), que correspondem às intensidades $I / I_{\text {sat }}=0.67$ e $I / I_{\text {sat }}=0.90$, respectivamente. Ao incrementar a potência, as ressonâncias nas bandas laterais se alargam, tornando os campos anti-correlacionados para um intervalo de dessintonias $|\delta|>\Delta \omega_{\text {Int }}$.

Na ref.[89] publicamos os resultados acima discutidos sobre a espectroscopia de correlação para casos não ressonantes. Assim, apresentamos então os espectros de correlação nas figuras $(4.10,4.12,4.13$ e 4.14$)$ para diferentes dessintonias de um fóton de um sistema $\Lambda$-EIT, contrastados com o modelo teórico que permitiu realizar uma mapeamento da correlação de intensidade com a resposta atômica no domínio das frequências. Esse mapeamento se caracteriza principalmente pelas propriedades de absorção e dispersão do meio com relação aos campos incidentes. Uma primeira interpretação dos resultados obtidos pode ser feita 
analisando as escalas espectrais com relação à largura natural, e pode dar uma visão geral dos resultados. A segunda interpretação pode ser colocada em termos das defasagens induzidas pelo meio, aprofundando-nos no mapeamento dispersão-correlação. Começaremos pela interpretação de escalas espectrais. Dentro destes resultados conseguimos distinguir três cenários diferentes para detectar as ressonâncias nas frequências das bandas laterais. $\mathrm{O}$ primeiro é o caso ressonante $\left(\Delta_{1}=0\right)$ da figura 4.15(a), onde as três situações de dessintonia (1), (2) e (3) são dominadas pela emissão espontânea e estimulada, já que a emissão dos átomos popula as densidades espectrais dos campos no intervalo de frequências $|\delta|<\Gamma$, sem resolver as ressonâncias nas bandas laterias.

(a)

1

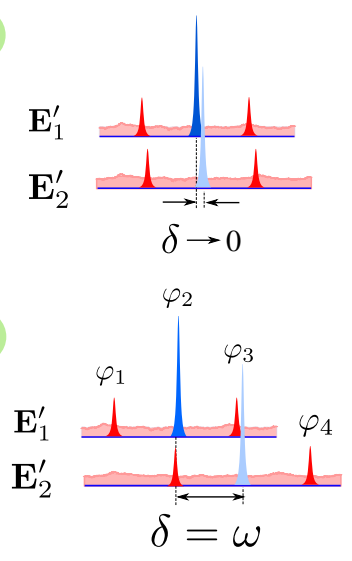

3

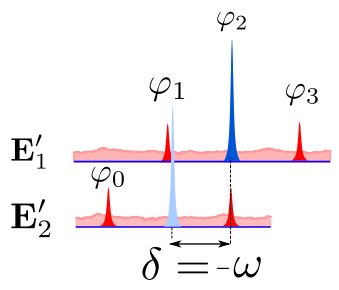

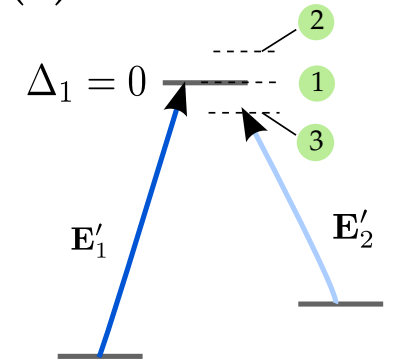

(b)

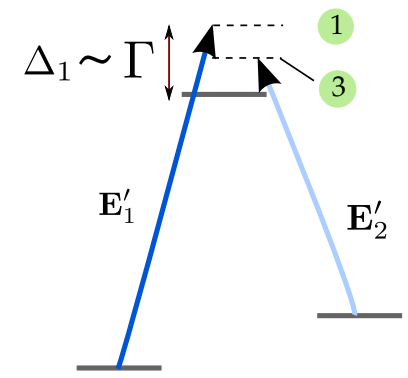

Figura 4.15: Descrição dos principais cenários da espectroscopia de correlação. As três principais situações são: (caso 1) condição de transparência quando $\delta \sim 0$, (caso 2) quando a portadora do campos $\mathbf{E}_{2}$ é ressonante com a banda lateral superior do campo $\mathbf{E}_{1}(\delta \sim \omega)$ e (caso 3) quando a portadora é ressonante com a banda lateral inferior $(\delta \sim-\omega)$. Esses processos podem ser analisados para dois casos de dessintonia de um fóton: (a) Caso ressonante $\Delta_{1}=0$ e (b) caso não ressonante $\Delta_{1} \sim \Gamma$.

Entretanto, ao aplicar dessintonias de um fóton da ordem da largura natural do meio 
atômico, neste caso $\Delta_{1} \sim \Gamma / 2 \pi \sim 6 \mathrm{MHz}$ (ver figura 4.12(b)), nas situações (2) e (3) as ressonâncias das bandas laterais são resolvidas quando temos $|\delta| \sim \omega>\Delta \omega_{E I T}$. Em outras palavras, nas situações (2) e (3) obtemos as ressonâncias $\delta= \pm \omega$ no espectro da correlação devido ao batimento entre as portadoras (representadas pelos picos intensos centrais em azul) e uma das bandas laterais (representadas pelos picos vermelhos). Assim, neste segundo cenário, as ressonâncias são resolvidas, pois elas estão espectralmente afastadas da influencia da emissão espontânea e da emissão estimulada. Portanto, diferentes ressonâncias são acessadas simplesmente mudando a frequência de análise. Tais ressonâncias parecem ser insensíveis ao ruído de fase, como foi observado na figura 4.12(f), pois não são alteradas de forma significativa quando consideramos termos de ordem superior no modelo perturbativo. Cabe notar que estas ressonâncias são resolvidas pelo fato de empregar átomos frios. Com átomos à temperatura ambiente, o alargamento Doppler impossibilitaria a resolução de tais ressonâncias, análogo ao caso da figura (a).

O terceiro cenário que analisaremos corresponde ao de alargamento por potência das ressonâncias nas bandas laterais (ver figuras. 4.14) . Ao aumentar a potência dos campos, o alargamento devido à saturação do meio atinge primeiramente as ressonâncias mais próximas da ressonância atômica (caso 3 da figura 4.15.(b)). Entretanto, ao aumentar a saturação, o alargamento atinge as ressonâncias mais afastadas (caso 2 da figura 4.15.(b)). Assim, identificamos os três cenários nos quais a relação das dessintonias com respeito à largura natural revela a influência da emissão espontânea na resolução das ressonâncias nas bandas laterais.

Agora discutiremos a interpretação em termos de defasagens dos campos. Nela aproveitamos a abordagem linearizada do campo eletromagnético da eq. (3.42) para entender as características chaves dos mecanismos envolvidos nesta espectroscopia. Primeiro, podemos interpretar a difusão de fase como uma modulação de fase $\bar{\gamma}$ no limite em que ela é pequena com relação à dinâmica atômica (princípio do tratamento perturbativo). Quando medimos as componentes espectrais da fotocorrente $I(t)$ (gerada por campos intensos e próximos aos descritos por uma onda monocromática) pela sua transformada de Fourier $I(\omega)$, estamos investigando batimentos das bandas laterais com a parte mais intensa do campo. Para uma 
frequência de análise em particular $\omega$, o campo de interesse evolve três componentes: a portadora com frequência ótica $\omega_{i}$ e duas bandas laterais deslocadas em $\pm \omega$ com respeito à portadora. A partir da eq. (3.42), os campos podem ser descritos por

$$
\mathbf{E}_{i}^{\prime}(t)=\mathcal{E}^{\prime}(t)_{i} \exp \left(i \omega_{i} t\right) \mathbf{e}_{i}
$$

com uma amplitude complexa dada por

$$
\mathcal{E}^{\prime}(t)_{i}=\mathcal{E}_{i}+\mathcal{E}_{i}^{u} \exp (i \omega t)+\mathcal{E}_{i}^{l} \exp (-i \omega t)
$$

sendo esta a abordagem linearizada da difusão de fase. Uma modulação pura de amplitude ocorre quando as bandas superiores e inferiores são simétricas e conjugadas $\left(\mathcal{E}_{i}^{u}=\left[\mathcal{E}_{i}^{l}\right]^{*}\right)$. Entretanto, uma modulação pura de fase ocorre quando rotamos em $\pi / 2$ a fase com respeito à amplitude da portadora $\mathcal{E}_{i}\left(\mathcal{E}_{i}^{u}=-\left[\mathcal{E}_{i}^{l}\right]^{*}\right)$. Conversão nas flutuações de fase e amplitude são possíveis se deslocamos assimetricamente as amplitude das bandas laterais $\left(\mathcal{E}_{i}^{u}, \mathcal{E}_{i}^{l}\right)$ ou deslocamos a fase da amplitude da portadora $\mathcal{E}_{i}$ [88] (técnica já descrita na seção 4.1.3). No nosso sistema, os átomos podem induzir tais deslocamentos assimétricos na amplitude (absorção) e na fase (dispersão). O que observamos no nosso experimento é então a transformação de ruído de fase para ruído de amplitude (RF-RA)

Levando em conta o que foi descrito acima, ao analisarmos o caso ressonante $\left(\Delta_{1} \simeq 0\right)$, a estrutura central da correlação $C(\omega)$ que está em condição de transparência $\left(|\delta|<\Delta \omega_{E I T}\right)$, e que é similar em traços gerais à função $g^{(2)}(0)$, é consequência do deslocamento na fase dado pelas portadoras enquanto varre a ressonância de EIT (ver figura. 4.15.a). Esse deslocamento converte o ruído de fase em ruído de amplitude, com sinais opostos para os campos 1 e 2 (como foi discutido na figura 3.11), induzindo um aumento na anticorrelação dos campos ao aumentar as intensidades, se impondo sobre qualquer pequena correlação que os campos possam ter originalmente. Esta estrutura central da correlação, como vimos anteriormente, é caracterizada pela largura intrínseca, tanto no domínio do tempo quanto no domínio da frequência. Neste caso, as bandas laterais são quase afetadas simetricamente pelo processo 
RF-RA: a banda lateral superior (inferior) de ambos os campos possuem a mesma defasagem e a mesma atenuação (transformações similares para $\mathcal{E}_{1}^{u}$ e $\mathcal{E}_{2}^{u}$ ), induzindo um sinal de correlação. As ressonâncias nas bandas laterais nos caso 2 e 3 não são resolvidas devido à emissão espontânea e à emissão estimulada, como mencionamos anteriormente.

As ressonâncias nas bandas laterais serão evidentes no caso em que a dessintonia de um fóton seja da ordem da largura natural atômica (ver figura 4.15.b). Mais uma vez, próximo da condição de transparência $|\delta|<\Delta \omega_{E I T}$ (caso 1), as bandas laterais sofrem os mesmos deslocamentos em fase e amplitude, induzindo máxima correlação para $|\delta| \simeq 0$, como no caso da figura (a). A novidade neste caso, é o fato que quando a dessintonia coincide com a frequência de análise $(|\delta|=\omega)$, a correlação é reduzida. Consideremos então a modulação de fase da eq. (4.10), onde $\mathcal{E}_{i}^{u}=-\left[\mathcal{E}_{i}^{l}\right]^{*}=\alpha$. A transformação de fase para o feixe que mantém sua dessintonia fixa é dada por:

$$
\begin{aligned}
\mathcal{E}^{\prime}(t)_{1} & =\alpha e^{i \omega t}+\mathcal{E}_{1}-\alpha^{*} e^{-i \omega t} \rightarrow \\
\mathcal{E}^{\prime}(t)_{1} & =\alpha e^{i\left(\omega t+\varphi_{3}\right)}+\mathcal{E}_{1} e^{i \varphi_{2}}-\alpha_{1}^{*} e^{-i\left(\omega t-\varphi_{1}\right)}= \\
& =\left[\alpha e^{i\left(\omega t+\varphi_{3}-\varphi_{2}\right)}+\mathcal{E}_{1}-\alpha^{*} e^{-i\left(\omega t+\varphi_{2}-\varphi_{1}\right)}\right] e^{i \varphi_{2}}
\end{aligned}
$$

Portanto, diferentes deslocamentos em fase vão converter ruído de fase em ruído de amplitude.

Ao aplicar a mesma transformação ao segundo campo (aquele que esta varrendo), quando a sua portadora está ressonante com a banda lateral superior do campo fixo (caso 2), temos

$$
\begin{aligned}
\mathcal{E}^{\prime}(t)_{2} & =\alpha e^{i \omega t}+\mathcal{E}_{2}-\alpha^{*} e^{-i \omega t} \rightarrow \\
\mathcal{E}^{\prime}(t)_{2} & =\alpha e^{i\left(\omega t+\varphi_{4}\right)}+\mathcal{E}_{2} e^{i \varphi_{3}}-\alpha^{*} e^{-i\left(\omega t-\varphi_{2}\right)}= \\
& =\left[\alpha e^{i\left(\omega t+\varphi_{4}-\varphi_{3}\right)}+\mathcal{E}_{2}-\alpha^{*} e^{-i\left(\omega t+\varphi_{3}-\varphi_{2}\right)}\right] e^{i \varphi_{3}} .
\end{aligned}
$$

Podemos notar que ao desconsiderar a fase global $\varphi_{3}$ (não observada após a detecção de intensidade), o deslocamento efetivo sobre a banda lateral superior do campo $\mathcal{E}^{\prime}(t)_{1}$ é o conjugado do deslocamento de fase da banda inferior do campo $\mathcal{E}^{\prime}(t)_{2}$. As flutuações rotarão 
em sentidos opostos no diagrama de fresnel, resultando em uma anticorrelação entre os campos.

Agora, se a portadora do segundo campo é ressonante com a banda inferior do campo fixo (caso 3), a transformação será dada por

$$
\begin{aligned}
\mathcal{E}^{\prime}(t)_{2} & =\alpha e^{i \omega t}+\mathcal{E}_{2}-\alpha^{*} e^{-i \omega t} \rightarrow \\
\mathcal{E}^{\prime}(t)_{2} & =\alpha e^{i\left(\omega t+\varphi_{2}\right)}+\mathcal{E}_{2} e^{i \varphi_{1}}-\alpha^{*} e^{-i\left(\omega t-\varphi_{0}\right)}= \\
& =\left[\alpha e^{i\left(\omega t+\varphi_{2}-\varphi_{1}\right)}+\mathcal{E}_{2}-\alpha^{*} e^{-i\left(\omega t+\varphi_{1}-\varphi_{0}\right)}\right] e^{i \varphi_{1}} .
\end{aligned}
$$

O deslocamento em fase da banda lateral superior do campo $\mathcal{E}^{\prime}(t)_{2}$ será o conjugado do deslocamento de fase da banda lateral superior do campo $\mathcal{E}^{\prime}(t)_{1}$. Mais uma vez esse processos induzem anticorrelação no resultado de $C(\omega)$.

Esta conversão depende do ganho da fase de cada modo. O alargamento por potência que sofre a correlação para dessintonias maiores que a largura intrínseca [43, 44] aumenta a anticorrelação entre os campos, como aparece na figura 4.14, de acordo com a redução das diferenças de deslocamento de fase. Concluímos assim a interpretação dos três cenários estudados nesta seção.

\subsection{Forças de espalhamento na espectroscopia de correlação}

Ao longo da pesquisa sobre a espectroscopia de correlação, nos deparamos com o regime dinâmico detectado nos espectros como um sinal de "histerese". Nesta seção mostraremos a suas principais características, as duas causas e discutiremos algumas das suas consequências. 


\subsubsection{Assimetria do espectro de correlação com respeito a varredura de frequência}

Os espectros mostrados ao longo da seção 4.2 foram obtidos fazendo uma varredura linear da frequência para um dos campos, tal que $\frac{d \delta}{d t}>0$. No entanto, com intuito de otimizar o sinal de EIT, em que se busca a máxima transparência junto como a melhor densidade ótica, decidimos modular a dessintonia tal que $\frac{d \delta}{d t}<0$. Resultado: a correlação toma valores opostos segundo a varredura feita na dessintonia $\delta$. A figura 4.16.(a) e (b), mostra a espectroscopia da correlação e o sinal DC respectivamente. Em linha azul é traçado o espectro para o caso em que $\frac{d \delta}{d t}>0$, e em linha vermelha traçamos os espectros para $\frac{d \delta}{d t}<0$.
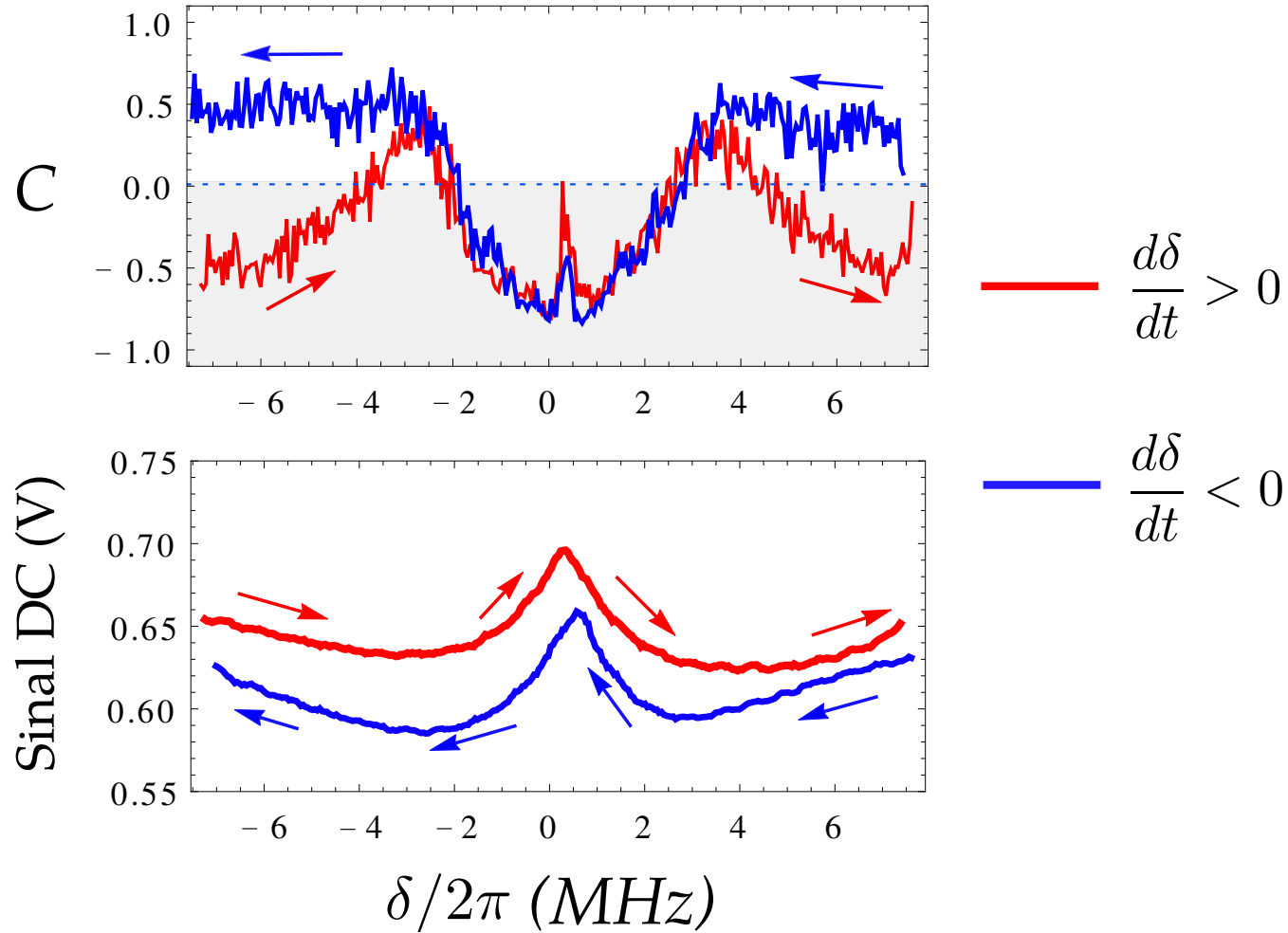

$\frac{d \delta}{d t}<0$

Figura 4.16: Espectroscopia de correlação (parte superior) e sinal DC (parte inferior) com varredura de frequência tal que $\frac{d \delta}{d t}<0$ (linha azul) $e \frac{d \delta}{d t}>0$ (linha vermelha).

A correlação na figura 4.16.(a), apresenta uma clara diferença entre as duas formas de varredura de frequência. Para uma varredura negativa $\left(\frac{d \delta}{d t}<0\right)$ o espectro mostra um nível de anti-correlação $C<0$ numa região de dessintonia $|\delta|>3 \Delta \omega_{E I T}$ como foi observado na 
seção anterior, enquanto que para uma varredura positiva $\left(\frac{d \delta}{d t}>0\right)$, o espectro mostra uma correlação positiva. Entretanto o sinal DC na figura 4.16.(b) não apresenta uma assimetria significativa entre os dois tipos de varredura, a partir da qual pudesse-se inferir algum grau de "histerese".

Este efeito de histerese na correlação não era esperado para este tipo de sistemas, nem tampouco há referências de trabalhos que já tenham observado este tipo de efeito. Isto motivou um estudo mais detalhado para explicar as causas do fenômeno. Começamos descartando qualquer erro de instrumentação, para ter certeza que é um efeito puramente induzido pelos átomos. A única possibilidade relacionada à instrumentação poderia ser algum tipo de atraso nos AOMs quando mudamos o sentido em que fazemos a varredura de frequência. Dado que o controle dos AOMs é feito por meio do computador, se a variação temporal de tensão no sinal de saída do computador não possui uma resposta linear no AOM (por exemplo uma resposta quadrática), isto poderia mudar a resposta atômica. No entanto, verificamos que os AOMs respondem tão rápido como o sinal de saída do computador.

Então começamos analisar as características espectrais da correlação para ambos tipos de varredura. Em particular, analisamos os espectros de correlação para as dois tipos de varredura e o espectro discreto para 40 pontos de dessintonia, como mostra a parte superior da figura 4.17. Os espectros são acompanhados da evolução temporal da correlação para três casos particulares de dessintonia de dois fótons. No caso do espectro discreto, podemos notar que a correlação toma valores próximos ao caso de varredura positiva ou negativa, dependendo da dessintonia ao vermelho $(\delta<0)$ ou ao azul $(\delta>0)$, respectivamente. Para o caso de varredura positiva em que os campos inicialmente são dessintonias ao vermelho, o espectro discreto mostra que o sistema apresenta um regime de anticorrelação. No entanto, para valores de dessintonia ao vermelho o espectro discreto parece coincidir com o espectro de varredura positiva, onde o sistema apresenta correlação. Cabe notar que dentro da janela de transparência $|\delta|<3 \times \Delta \omega_{E I T}$ (representada pelas linhas verticais pontilhadas) não há diferenças entre ambos tipos de varredura e o espectro discreto.

Ao realizar a espectroscopia discreta, percebemos que era importante analisar a evolução 
(1)

(2)

(3)

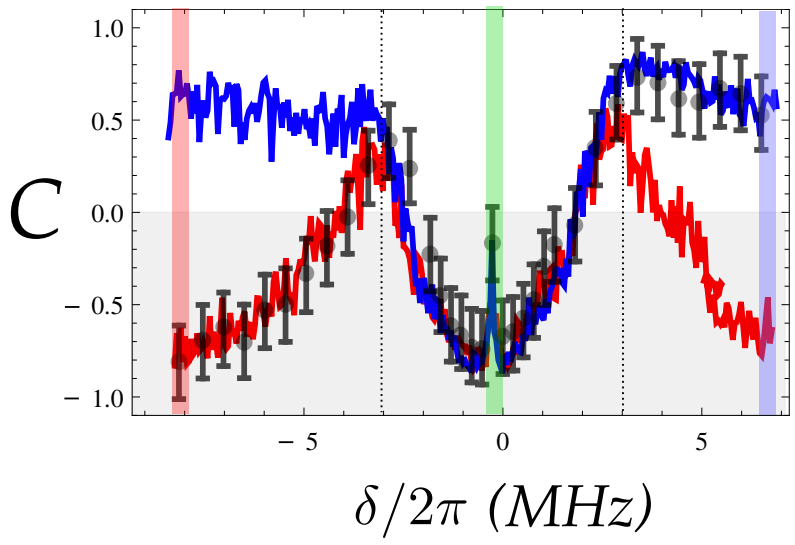

(1)
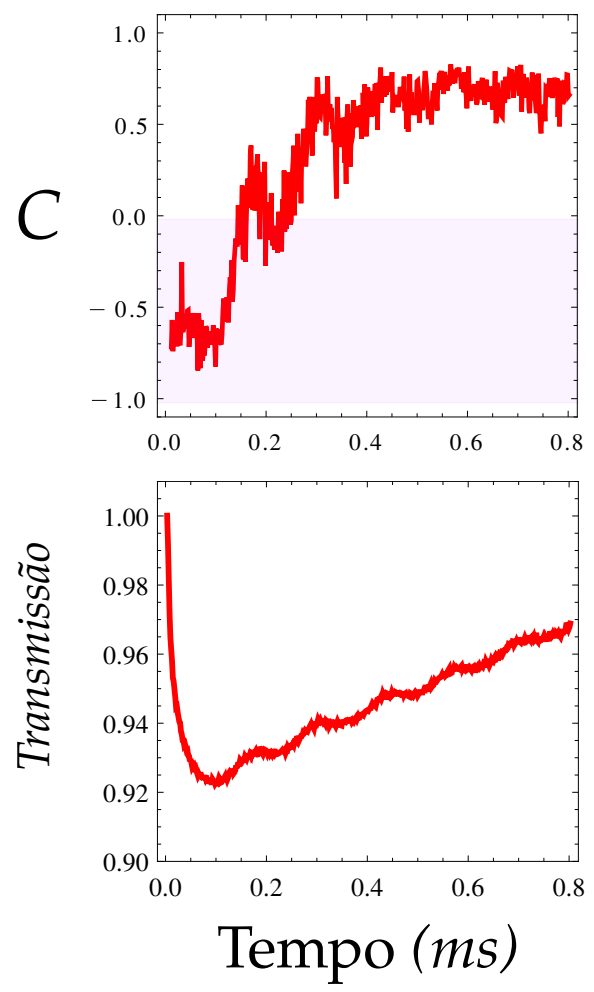

(2)
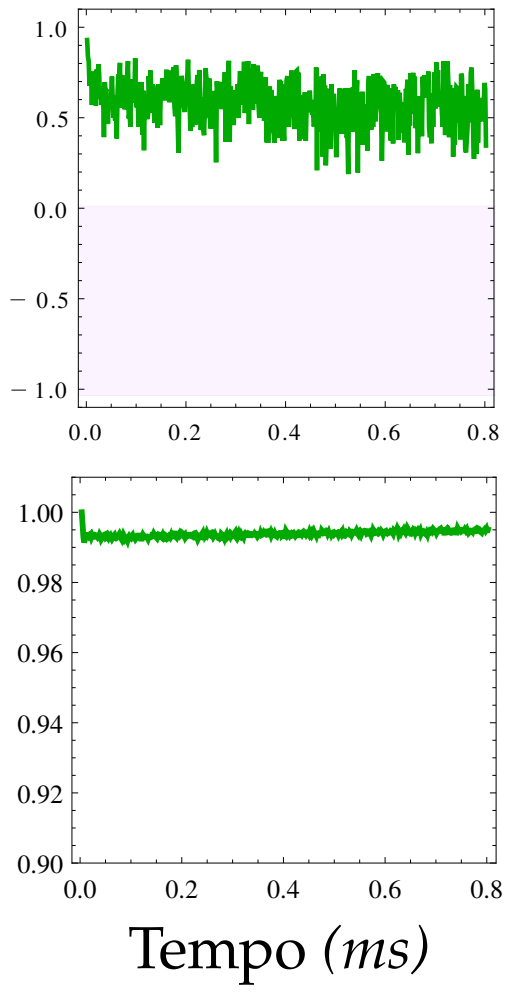

(3)
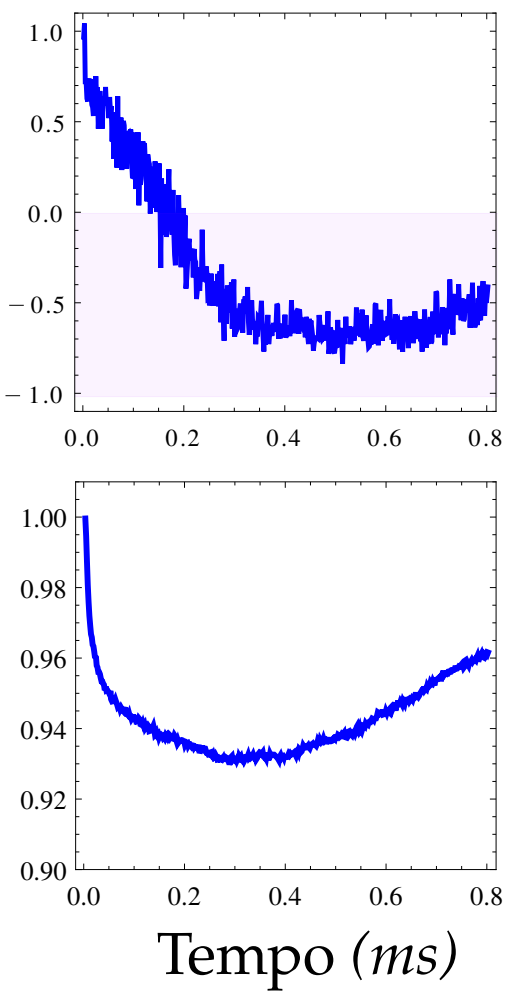

Figura 4.17: Espectros de correlação contínuo (em linha azul e vermelha) e discreto (os pontos pretos). Os espectros foram tomados com uma dessintonia do rebombeio de $\Delta_{\text {Reb }}=0 \mathrm{MHz}$ e com uma potência de $600 \mu \mathrm{W}$ em ambos os feixes de prova. Para os dois espectros se mostra a evolução temporal da correlação para três valores de dessintonia de dois fótons (1) $\delta=-7 \mathrm{MHz}$, (2) $\delta=0 \mathrm{MHz}$ e (3) $\delta=7 \mathrm{MHz}$. As linhas verticais pontilhadas representam a janela $3 \times \Delta \omega_{E I T}$. 
temporal da correlação para os pontos do espectros discreto. Decidimos então analisar três pontos de dessintonia da correlação, segundo mostra a figura 4.17. Os pontos (1) e (3) para as dessintonias $\delta=-7 \mathrm{MHz}$ e $\delta=7 \mathrm{MHz}$, onde claramente o efeito de histerese está presente no sistema. Neles, podemos notar que a correlação apresenta uma evolução temporal transiente tal que o sistema toma um tempo de $\sim 0.4 \mathrm{~ms}$ para chegar numa condição estacionária. Já para o caso de dessintonia $\delta=0 \mathrm{MHz}$, a correlação se mantém na condição estacionária, sem apresentar nenhum tipo de evolução transiente. Assim temos identificado uma das características mais importantes: a evolução transiente da correlação implica uma assimetria espectral da correlação com respeito ao tipo de varredura, para $|\delta|>3 \times \Delta \omega_{\text {EITT }}$.

No que diz respeito ao sinal DC da intensidade, podemos notar que a absorção dos campos também apresenta diferentes comportamentos segundo a dessintonia $\delta$, como mostra a figura 4.17. Em particular observamos que a evolução temporal para $\delta=-7 \mathrm{MHz}$ e $\delta=7$ MHz é assimétrica. Aqui notamos a segunda característica importante do sistema: supondo que o nosso sistema atômico é um sistema de três níveis fechado, pela solução das equações de Bloch, a evolução temporal da absorção dos campos não é assimétrica com relação à dessintonia de dois fótons. Cabia se perguntar qual era a causa de tal assimetria e da longa escala que apresenta a correlação e o sinal DC.

Decidimos então analisar a evolução temporal das variáveis atômicas considerando os vários níveis atômicos envolvidos na espectroscopia dos átomos. Os cálculos foram feitos por Carlos González (aluno de doutorado) e alguns deles são apresentado no apêndice B. A partir desses cálculos, os quais mostram que os graus internos atômicos atinge seu estado estacionário numa escala de tempo máxima de $\mu \mathrm{s}$, chegamos à conclusão de que a escala de tempo (da ordem de ms) com que a correlação evolui para cada um desses pontos de dessintonia, pode ser unicamente devido aos graus externos do átomo (posição ou momento), descartando qualquer efeito intrínseco na evolução dos graus internos (evolução temporal dos níveis atômicos em presença da interação com os campos em EIT). 


\subsubsection{Efeito Doppler induzido pelas forças de espalhamento da luz sobre átomos}

Dada a assimetria espectral na absorção dos átomos na figura 4.17, eu propus a hipótese de que a sua causa poderia ser devido às forças de espalhamento que os feixes exercem nos átomos. A hipótese era a seguinte: se os campos estiverem exercendo força nos átomos, ao ganharem velocidade, se induziria um deslocamento Doppler na frequência dos campos, como foi discutido na seção 2.4.1. Isto significa que, de acordo com a eq.(2.22), cada campo ao interagir com os átomos exerce uma força de espalhamento $\mathbf{F}_{i}=F_{R} \hat{\mathbf{k}}_{i}$ onde $F_{R}$ e proporcional absorção da luz, e $\hat{\mathbf{k}}_{i}$ é a direção de propagação da luz ${ }^{3}$, sendo $i=1,2$ (ver figura 4.18). Portanto, a velocidade que o átomo adquiri é $\mathbf{v}(t)=\mathbf{v}_{0}+\left(\mathbf{F}_{1}+\mathbf{F}_{2}\right) t / m$, e assim o deslocamento Doppler induzido em cada campo é $\mathbf{k}_{i} \cdot \mathbf{v}(t)$. Assim, ao fazer a espectroscopia, dependendo do intervalo de dessintonias, devido às forças de espalhamento dos campos, se teriam um deslocamento no tempo das variáveis sendo analisada (absorção ou correlação de intensidade).
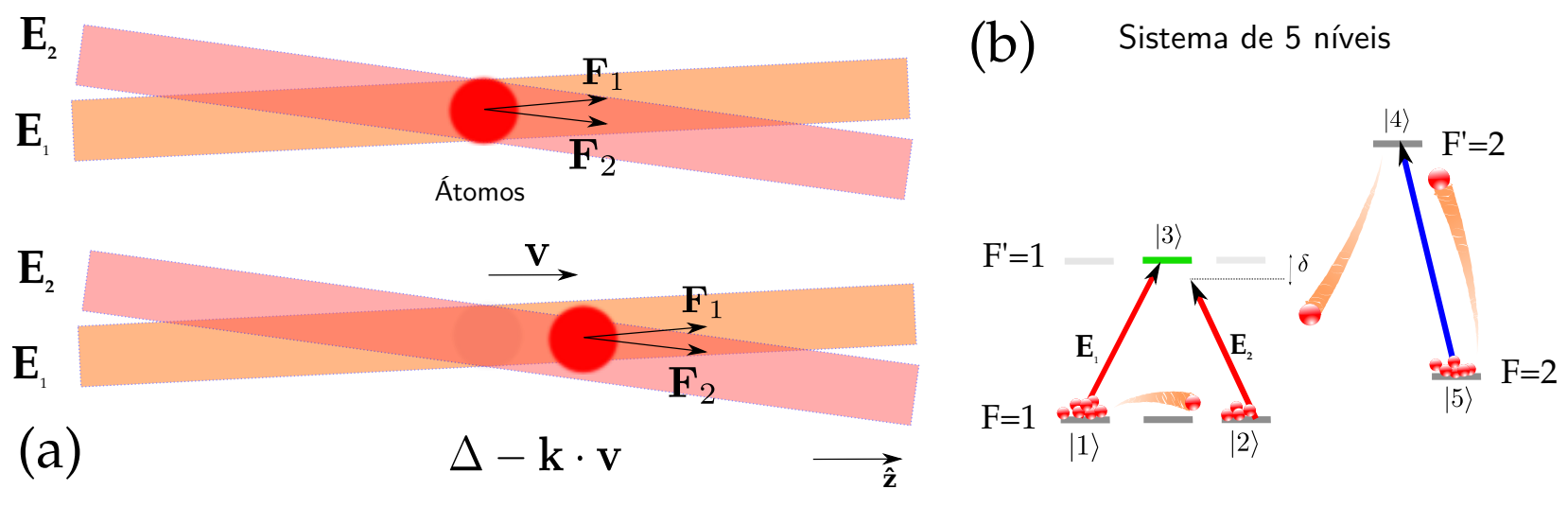

Figura 4.18: (a) Forças de espalhamento atuando sobre os átomos, induzindo um deslocamento Doppler na frequência de $\mathbf{k} \cdot \mathbf{v}$. (b) Sistema de cinco níveis.

Para constatar esta hipótese, consideramos teoricamente o efeito das forças na evolução

\footnotetext{
${ }^{3}$ Aqui ignoramos o efeito da força dipolar, pois o gradiente $\nabla I$ não é intenso suficiente para ter uma componente significativa na direção radial ao eixo do feixe. A relação da força de dipolo e da força de espalhamento é de $\sim 10^{-4}$ para os parâmetros experimentais.
} 
da dinâmica dos estados atômicos. Para isto, resolvemos o seguinte sistema de equações:

$$
\begin{aligned}
& \frac{d\langle\mathbf{x}(t)\rangle}{d t}=-\mathbf{M}_{5 n i v}[\mathbf{v}(t)]\langle\mathbf{x}(t)\rangle+\mathbf{x}_{0} \\
& m \frac{d \mathbf{v}(t)}{d t}=\mathbf{F}_{1}+\mathbf{F}_{2}
\end{aligned}
$$

onde a primeira equação descreve as equações de Bloch para as variáveis atômicas do sistema de cinco da figura 4.15.(b) e a segunda equação corresponde a equação de força sobre átomos. Para resolver o sistema, consideramos a velocidade inicial dos átomos como $\mathbf{v}(t=0)=0$, pois ao termos átomos frios, esta é uma aproximação valida. Entretanto, as forças são proporcionais à absorção de cada um dos campos, isto é, $\mathbf{F}_{i} \propto \operatorname{Im} \rho_{i 3}$. Dado o pequeno ângulo entre os feixes de prova, consideramos que a força $\mathbf{F}_{1}+\mathbf{F}_{2}$ se superpõem linearmente na na direção $\hat{\mathbf{z}}$, ignorando as componentes transversais, pois não há deslocamento Doppler nessa componente. A matriz dinâmica $\mathbf{M}_{5 n i v}(t)$ corresponde à matriz que descreve o nosso sistema completo de cinco níveis da figura 4.15.(b). Ela depende explicitamente da velocidade, pois agora consideramos que as dessintonias são dependentes de $\mathbf{k}_{i} \cdot \mathbf{v}$. As densidades espectrais também foram calculadas a partir da matriz espectral da eq.3.86, nesse caso considerando a matriz $\mathbf{M}_{5 n i v}$.

Levando em consideração que os campos exercem forças nos átomos, e como isto muda a dinâmica, decidimos estender a análise feita na figura 4.17 paras várias dessintonias para observar a evolução temporal e a assimetria espectral da correlação e da transmissão, como mostram as figuras 4.19(a) e (c). Além disso, a figura 4.19(b) e (d) mostra a solução numericamente das eqs. $(4.14,4.15)$ e da correlação teórica dos campos, calculada pelo Carlos. Cabe notar que tanto as medidas quanto os cálculos, levam em consideração as forças exercidas pelos campos de prova de EIT e o campo de rebombeio, que é contrapropagante aos de prova.

Podemos notar como a correlação e a transmissão se torna assimétrica com relação a dois pontos de dessintonia opostos, à medida que ela aumenta. No caso das dessintonias $\delta \sim \pm 1$ $\mathrm{MHz}$, as quais estão dentro da janela de transparência (ver figura 4.17), podemos observar 

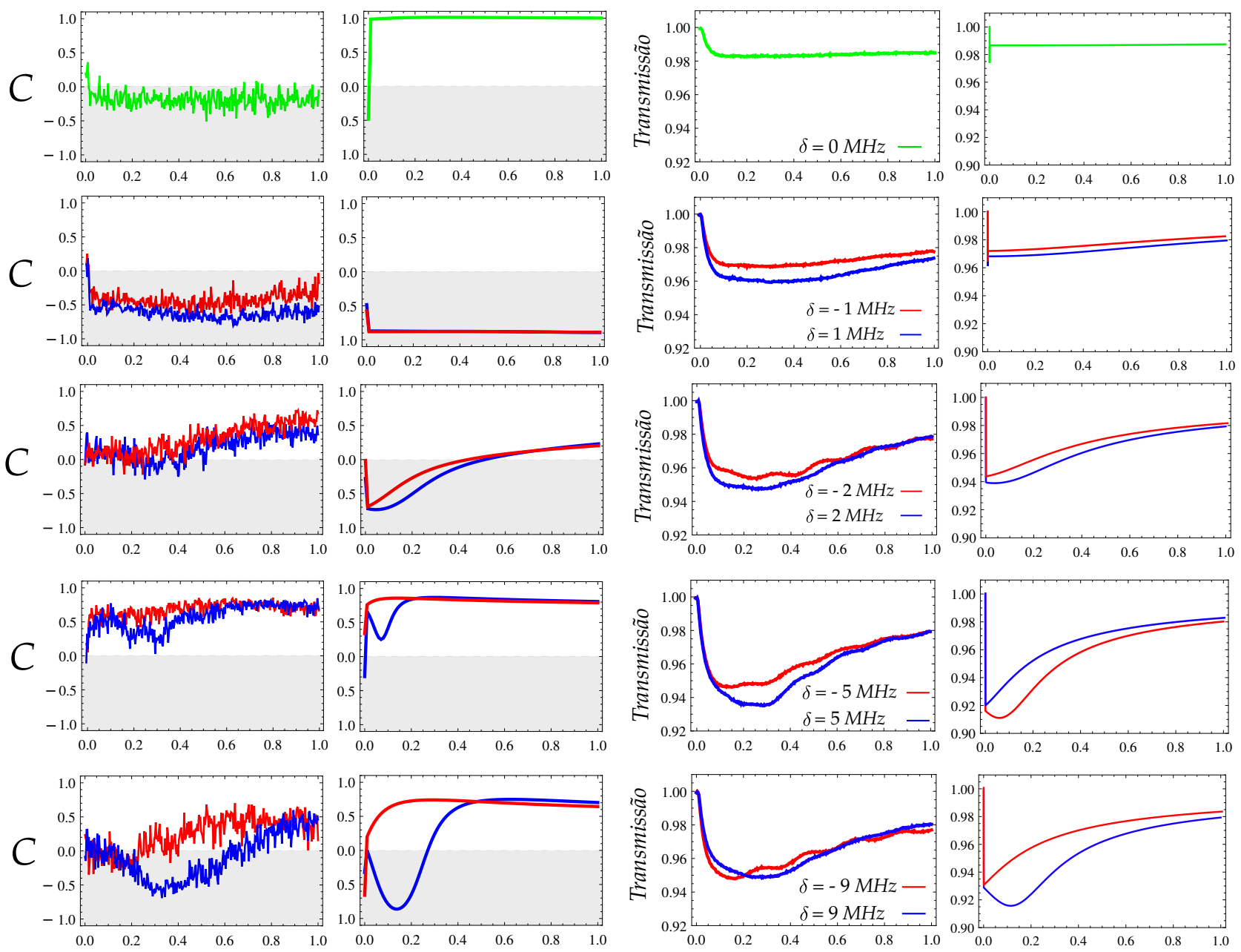

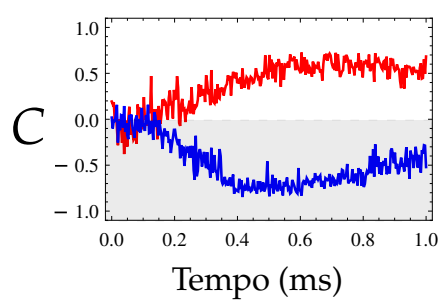

(a)

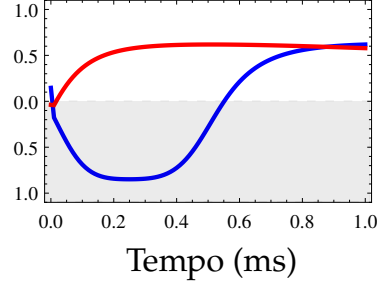

(b)

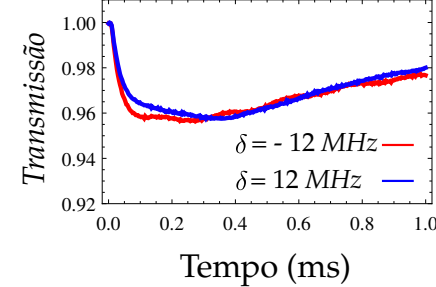

(c)

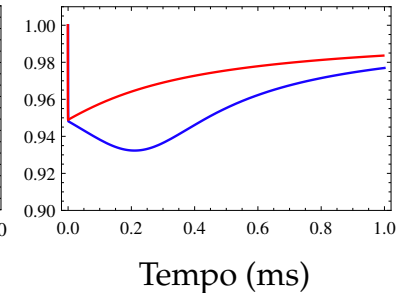

(d)

Figura 4.19: Evolução temporal da correlação de intensidade e da transmissão. (a) e (b) corresponde a correlação de intensidade experimental e teórica, respectivamente. (c) e (d) corresponde a transmissão experimental e teórica, respectivamente.

que a correlação e a transmissão apresentam uma taxa de evolução transiente muito menor que para os casos dessintonizados. Isto quer dizer, que à medida que que os campos estão próximas de janela de transparência $|\delta|<2 \mathrm{MHz}$, o sistema é insensível ao efeito das forças que produzem uma evolução considerável para dessintonias fora dessa janela. Isto 
é consequência da transparência do meio atômico aos campos de luz, pois se as forças são proporcionais à absorção $\mathbf{F}_{i} \propto \operatorname{Im} \rho_{i 3}$, é de esperar que dentro da janela de transparência em que $\operatorname{Im} \rho_{i 3} \sim 0$ então as forças de espalhamento são nulas. Isto é consistente com o fato de que o espectro não apresenta "histerese"para $|\delta|<2 \mathrm{MHz}$, na figura 4.17.

Conforme a dessintonia aumenta, a correlação e a transmissão apresentam uma assimetria espectral significativa. Um claro exemplo disso é o caso de dessintonia $\delta= \pm 12 \mathrm{MHz}$. Também é importante destacar o ponto de transição para $\delta=5 \mathrm{MHz}$, em que a correlação é positiva ao longo da evolução, enquanto que para dessintonia vizinhas $\delta=2 \mathrm{MHz}$ e $\delta=9$ $\mathrm{MHz}$, a evolução passa por transição de correlação negativa para positiva. No que diz ao respeito do modelo teórico, o efeito das forças na evolução da correlação e transmissão dos campos revela uma descrição bastante satisfatória dos resultados experimentais. Cabe notar, que para este cálculo consideremos a força gerada pelo ponto máximos de um feixe com distribuição de intensidade gaussiana. Também consideremos que os dois campos se superpõem exatamente na posição da nuvem. Claramente, se estas condições sofrer pequenas alterações, as forças que atuam sobre os átomos podem gerar uma maior ou menor taxa evolução transiente. Suposições sobre esses dois parâmetros, fazem com que a taxa de evolução transiente do resultado teórico se ajuste a taxa do resultado experimental.

Dado que o efeito observado corresponde à dessintonia dos campos induzida pelo efeito Doppler devido à pressão de radiação, poderíamos descrever esse fenômeno sem precisar resolver as equações dinâmicas de um sistema de 5 níveis. De forma equivalente, poderíamos considerar a solução estacionária do sistema de três níveis da eq.(3.120), analisando a correlação para várias dessintonias de um fóton $\Delta<0$ (dessintonia ao vermelho pelo fato dos átomos serem empurrados pela luz), tomando $\Delta_{2}=\Delta+\delta$ e $\Delta_{1}=\Delta$.

A figura 4.20 mostra a correlação para os mesmos pontos de dessintonia da figura 4.19, comparando o modelo que leva em conta as forças dos campos no sistema de 5 níveis, e a solução estacionaria da eq.(3.120) em função da dessintonia de um fóton $\Delta$. Podemos concluir que o efeito dinâmico produzido pela força de radiação que empurra os átomos (considerando o sistema de 5 níveis), pode ser descrito por um sistema mais simples considerando unicamente a solução estacionária de um sistema de três níveis. Como tinha sido 
notado na figura 4.17, a evolução temporal da correlação e da transmissão é aquela que determina a aparente "histerese"na figura 4.16, No entanto, o efeito observado não se tratava de um efeito de propriamente de histerese ou de memoria dos átomos na hora de fazer a espectroscopia, mas sim, da influência sobre os átomos pelas forças exercidas da luz.
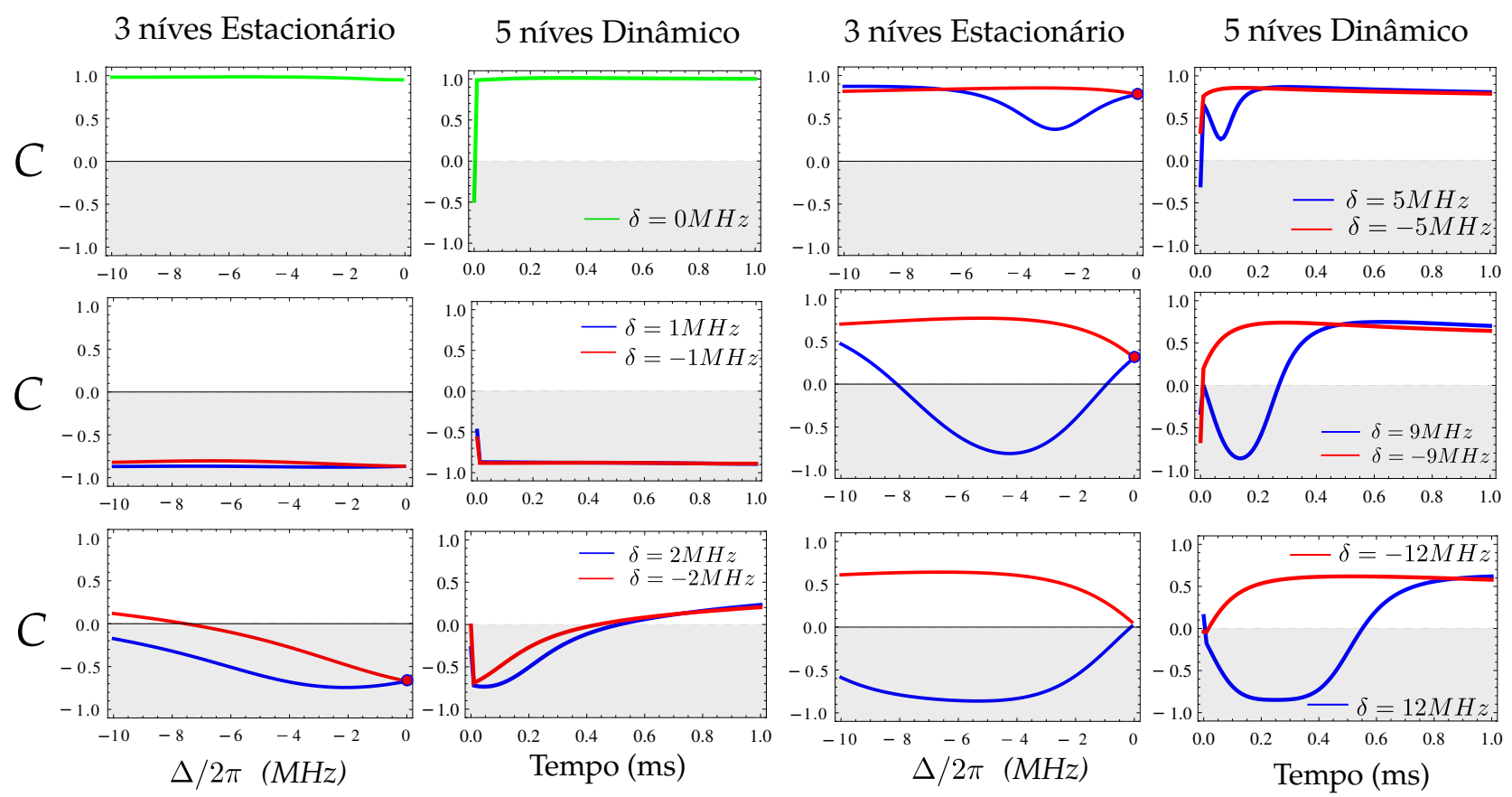

Figura 4.20: Comparação da correlação em função da dessintonia $\Delta$ e do tempo sob o efeito das forças que geram uma evolução temporal.

\subsubsection{Correlação em geometria contra-propagante}

Para testar a nossa hipótese sobre as forças da luz nos átomos, decidimos testar uma configuração geométrica oposta. Os espectros apresentados na seção anterior foram tomas na configuração co-propagante dos campos como mostra a figura 4.21(a). Nesta seção mostraremos a espectroscopia obtida numa configuração geométrica contra-propagante como mostra a figura 4.21(b). Dessa forma, se a intensidade dos campos fossem iguais, neste caso, as forças dos campos são opostas, cancelando então o efeito dinâmico. Desta forma seria de esperar que não tivéssemos nenhuma assimetria espectral na correlação.

Com esta nova configuração fizemos espectros de correlação para diferentes dessintonias 
Co- Propagante .

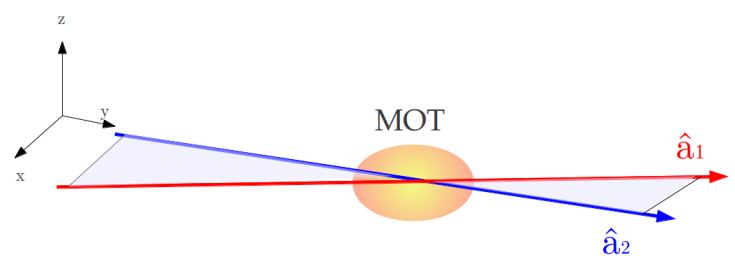

(a)

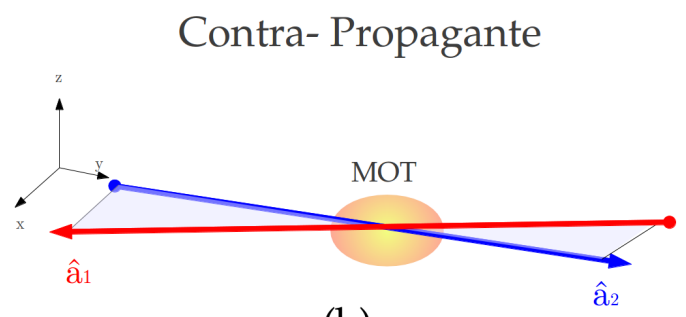

(b)

Figura 4.21: (a) Geometria co-propagante e (b) contra-propagante, entre os feixes de prova de EIT.

de um fóton do sistema $\Lambda$ e assim compará-los com os espectros obtidos anteriormente, no caso co-propagante da figura 4.11. A figura 4.22.(a) mostra as espectroscopias de correlação para a varredura temporal de dessintonia positiva (linha vermelha) e negativa (linha azul), com diferentes dessintonias $\Delta_{1}$. Observamos que os espectros apresentam o mesmo comportamento que no caso co-propagante da figura 4.11, ou seja, temos umas assimetria no espectro de correlação conforme nos afastamos da ressonância atômica $\Delta_{1}=0$. No entanto, a espectroscopia não apresenta nenhum efeito de "histerese"como foi observado na figura 4.16
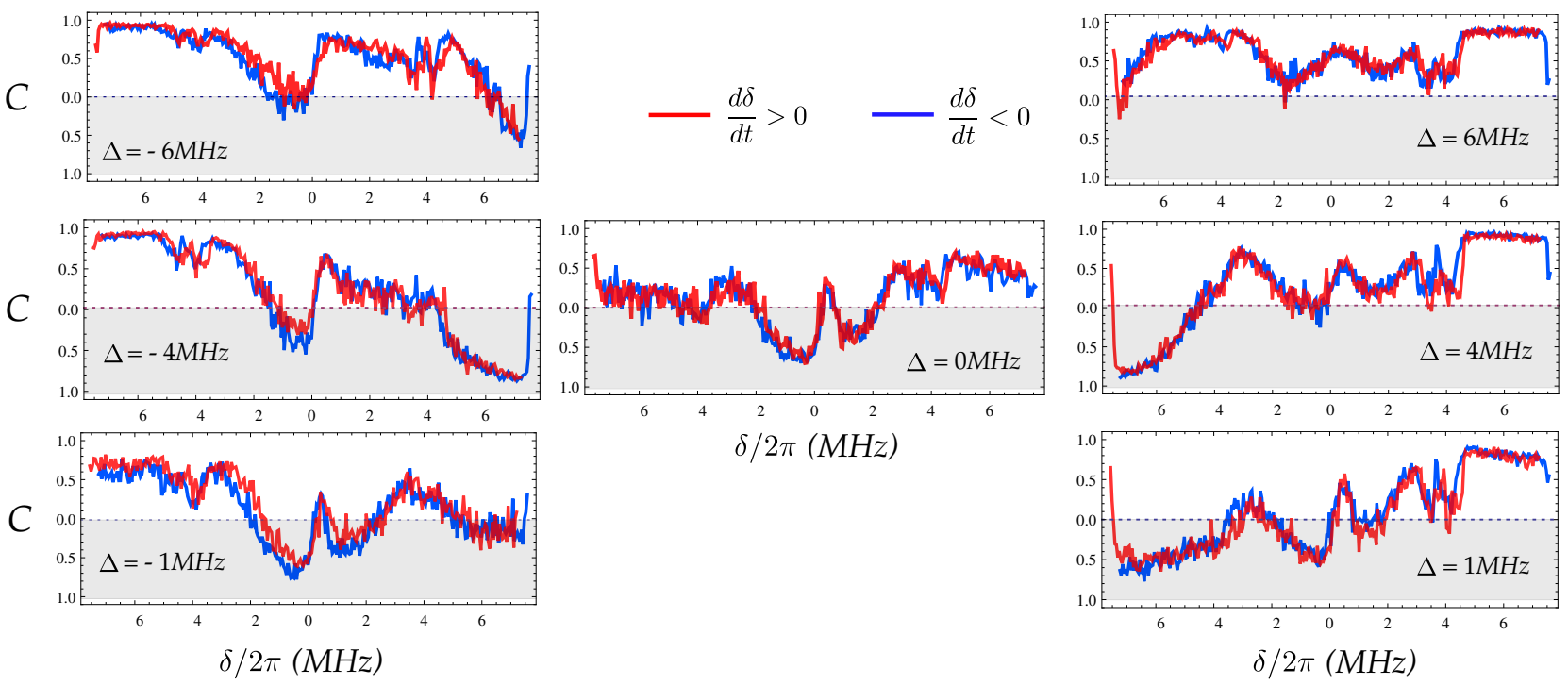

Figura 4.22: (a) Espectroscopia de correlação para $\Delta_{1}=-6,-4,-1,0,1,4,6 \mathrm{MHz}$ em geometria contra-propagante.

Portanto, mostramos que na configuração contrapropagante a forças dos feixes de prova 
se cancelam. Cabe notar que, a configuração contrapropagante, o sistema se torna sensível ao alargamento Doppler que pela temperatura da nuvem é da ordem de $500 \mathrm{kHz}$. Esse alargamento inhomogêneo, diminui a transparência dos campos [90] e, portanto, altera a resolução do espectro de correlação (esse espectros apresentam menor resolução do que o espectro da figura 4.16).

Isto indica certas considerações para serem levadas em conta para futuras aplicações dos átomos. Por exemplo, aqui no laboratório se pretende fazer interagir um dos campos emaranhados gerado por um oscilador paramétrico ótico (OPO), com os átomos frios em condição de EIT. O resultado mostrado nesta seção mostra que, se os átomos forem acoplados com tais campos fora da janela de transparência, a fidelidade do estado vai depender da assimetria espectral induzida pelas forças de espalhamentos. Entretanto, se o acoplamento se faz dentro da janela de transparência, o sistema seria insensível a o efeito dinâmico, e portanto se garante a fidelidade do estado de entrada da luz. Isto toma muita relevância desde o ponto de vista espectroscopia, já que pela espectroscopia da transmissão (sinal DC), detetar este tipo de assimetria espectral, pode escapar da simples observação. Isto coloca a espectroscopia de correlação como uma técnica o suficientemente precisa para detetar processo dinâmicos em relógios atômicos [91, 92].

\section{Conclusões do capítulo}

Ao longo deste capítulo fizemos uma análise experimental da correlação de intensidade no domínio da frequência $C(\omega)$ para dois campos interagindo com átomos frios de Rubídio em condição de EIT. Analisamos duas experiências em particular. A primeira, em que fazemos a espectroscopia de correlação para campos próximos da ressonância atômica e para campos com dessintonia $\sim \Gamma$. Isto mostrou que para os casos dessintonizados é possível resolver ressonâncias na frequência das bandas laterais que se caracterizam por uma clara tendencia à anticorrelação. Além disso, mostramos que o modelo perturbativo da seção 3.6 descreve de forma satisfatória os resultados experimentais. Com ele, foi possível fazer um mapeamento das variáveis atômicas, dispersão e absorção, com a correlação no domínio da frequência. Mostramos também, a função de correlação $g^{(2)}(0)$ do modelo heurístico mostrou-se como 
uma aproximação valida para descrever a correlação dos campos, quando os campos são dessintonizados até $\sim \Gamma$. No que diz respeito ao alargamento por potencias, mostramos que as ressonâncias das bandas laterais sofre tal alargamento. No entanto, para o caso ressonante, o coeficiente de correlação é influenciado pela largura natural atômica, de modo que as ressonâncias nas frequências da análise não são resolvidas.

Na segunda experiência analisamos o regime dinâmico dos espectros de correlação. Nele, se observou uma assimetria espectral com respeito à varredura de frequência dos lasers. Mostramos que essa assimetria é resultado do deslocamento em frequência devido ao efeito Doppler induzido pelas forças de espalhamento que a luz exerce sobre os átomos. Demostramos que para os intervalos de frequência fora da janela de transparência, a assimetria espectral se traduz numa evolução transiente da correlação e da transmissão dos campos. Entretanto, para frequências dentro da janela de transparência, o meio atômico se torna insensível a tais forças e, portanto, não apresenta uma assimetria espectral. Isto implica, que em qualquer aplicação futura que pretenda medir a correlação dos campos em condição de EIT, precisa se manter dentro da janela de transparência para evitar o efeito das forças. 


\section{CAPÍTULO 5 \\ CONCLUSÕES E PERSPECTIVAS}

Concluímos que com esse trabalho de doutorado chegamos em resultados teóricos e experimentais sobre espectroscopia de correlação, mostrando assim o estágio avançado da nossa técnica de espectroscopia de correlação. Em particular foram abordados três aspectos da espectroscopia de correlação. O primeiro deles é o aspecto teórico, em que propus um tratamento perturbativo para o cálculo da correlação no domínio da frequência para lasers com excesso de ruído de fase interagindo em condição de EIT com um átomo de três níveis. Mostramos como este tratamento é compatível com o tratamento feito pela solução de Itô adotada até então, o qual permite estabelecer uma relação direta entre a correlação de intensidades dos campos e os parâmetros de absorção e dispersão associados ao átomos, o que não tinha sido mostrado anteriormente. Isto foi muito importante, pois nos permitiu avançar no entendimento da resposta atômica para este tipo de sistemas, mostrando que o modelo heurístico [43] está contido na solução do método perturbativo.

Em conjunto com a análise teórica, fizemos um amplo estudo experimental sobre a espectroscopia de correlação de intensidades em condição de EIT. Na primeira parte mostramos que, para algumas condições particulares, a correlação resolve ressonâncias nas frequências das bandas laterais. Uma delas é quando os campos são dessintonizados da ressonância 
atômica e, portanto, é possível achar pontos de anticorrelação quando a dessintonia é ressonante com a frequência de análise da demodulação da fotocorrente. Além disso, mostramos que nesta condição a função de correlação $g^{(2)}(0)$ do modelo heurístico mostrou ser uma aproximação válida para descrever a correlação dos campos. Isto permite estender a interpretação da função $g^{(2)}(0)$, dada em termos da parte absortiva e dispersiva do átomo, para a correlação no domínio da frequência $C(\omega)$. Também se mostrou como o espectro é sensível a alargamento por potência e que, para o caso ressonante, o coeficiente de correlação é influenciado pela largura natural atômica, de modo que as ressonâncias nas frequências da análise não são resolvidas. Esta caracterização espectral da interface luz-átomos foi descrita de forma satisfatória pelo tratamento perturbativo proposto na primeira parte.

Na segunda parte do estudo experimental, analisamos o regime dinâmico para a espectroscopia de correlação. Neste regime se observou uma assimetria espectral na correlação com respeito à varredura da frequência dos lasers. Mostramos que essa assimetria é resultado do deslocamento em frequência devido ao efeito Doppler induzido pelas forças de espalhamento que a luz exerce sobre os átomos. Demostramos que para os intervalos de frequência fora da janela de transparência, a assimetria espectral se traduz numa evolução transiente da correlação e da transmissão dos campos. Entretanto, para frequências dentro da janela de transparência, o meio atômico se torna insensível a tais forças e, portanto, não apresenta uma assimetria espectral. Isto implica em que em qualquer aplicação futura que pretenda medir a correlação dos campos em condição de EIT, as frequências dos campos precisam se manter dentro da janela de transparência para evitar o efeito das forças.

Esses resultados motivam algumas perspectivas nos aspectos teóricos e experimentais da correlação de EIT. A primeira é considerar um terceiro campo no sistema de três níveis que esteja em ressonância com as bandas laterais da correlação, como a figura 5.1.(a). Com ele se pretende analisar a resposta na correlação das ressonâncias nas bandas laterais à perturbação do terceiro campo [93]. A segunda perspectiva é analisar o caso em que modulamos a fase de um dos campos mediante uma senóide [76], como mostra a figura 5.1.(b). As- 
sim poderíamos ver como as ressonâncias de Rabi afetam a correlação nas ressonâncias das bandas laterais. Aplicando o método de medida não destrutiva, poderíamos observar como essa modulação é medida na quadratura conjugada no campo complementar. Em particular, podemos analisar também o comportamento da ressonância das bandas laterais neste tipo de modulação. Uma terceira perspectiva é considerar campos coerentes em condição de EIT para observar se há efeitos quânticos nos ruído de fase.

(a)
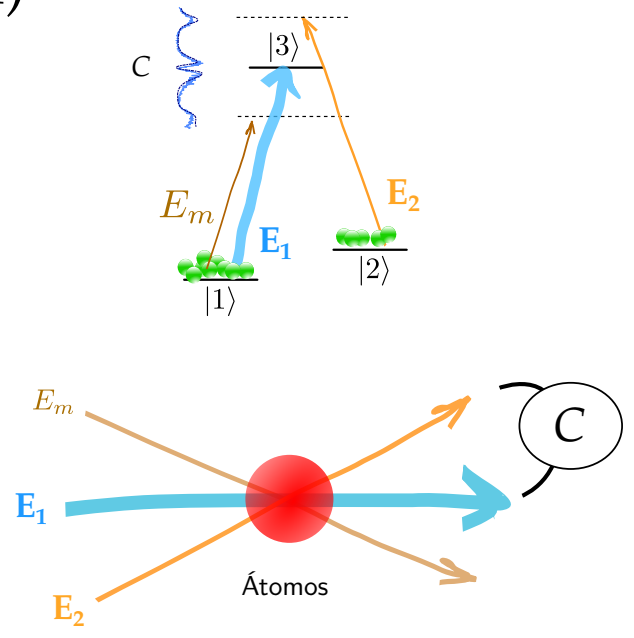

(b)
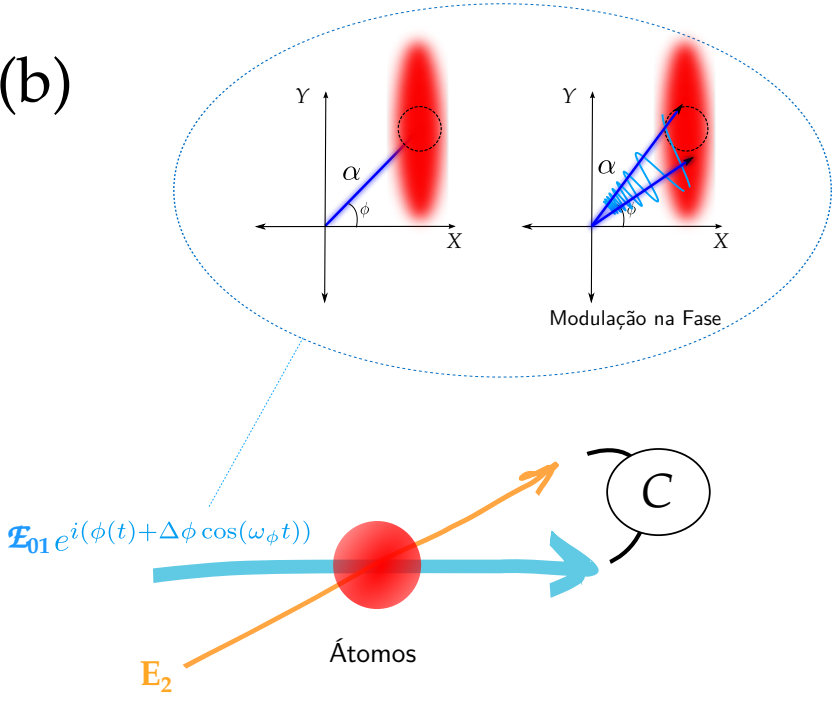

Figura 5.1: (a) Acoplamento do terceiro campo $E_{m}$ numa das ressonâncias das bandas laterais na correlação. (b) Modulação de fase num dos campos em condição de EIT.

Por outro lado, uma das perspectivas para a MOT é o acoplamento de campos emaranhados com átomos frios, projeto que está sendo desenvolvido no laboratório. Para isto pode ser empregado um OPO operando em $532 \mathrm{~nm}$ (ver a parte inferior da figura 5.2), ou o mais recentemente construído, operando em 780nm por um laser de Titânio safira (ver a parte superior da figura 5.2). Eles geram dois campos, sinal e complementar, emaranhados em $1061 \mathrm{~nm}$ (OPO em 532nm) e 1550nm (OPO em 780nm). A possibilidade de obter emaranhamento bipartite em $532 \mathrm{~nm}$ e tripartite em $780 \mathrm{~nm}$, potencializa a construção de uma interface quântica luz-átomo-chip, em que os átomos de Rubídio estão conectados com, por exemplo, um chip de silício que opera em um comprimentos de onda de $1550 \mathrm{~nm}$. Recentemente foi reportada a geração de estados comprimidos óticos neste tipo de dispositivos [94]. 
Portanto, uma interface luz-átomo-chip, em que dois sistemas de comprimentos de onda diferentes são conectado pelo emaranhamento produzido no OPO, projeta este sistema como um dos mais importantes para futuras aplicações em informação e comunicação quântica. Um exemplo disso seria a realização de teletransporte de um estado comprimido gerado pelo chip em 1550nm, para ser transmitido ao feixe de bombeio em 780nm, o qual pode interagir com os átomos frios.
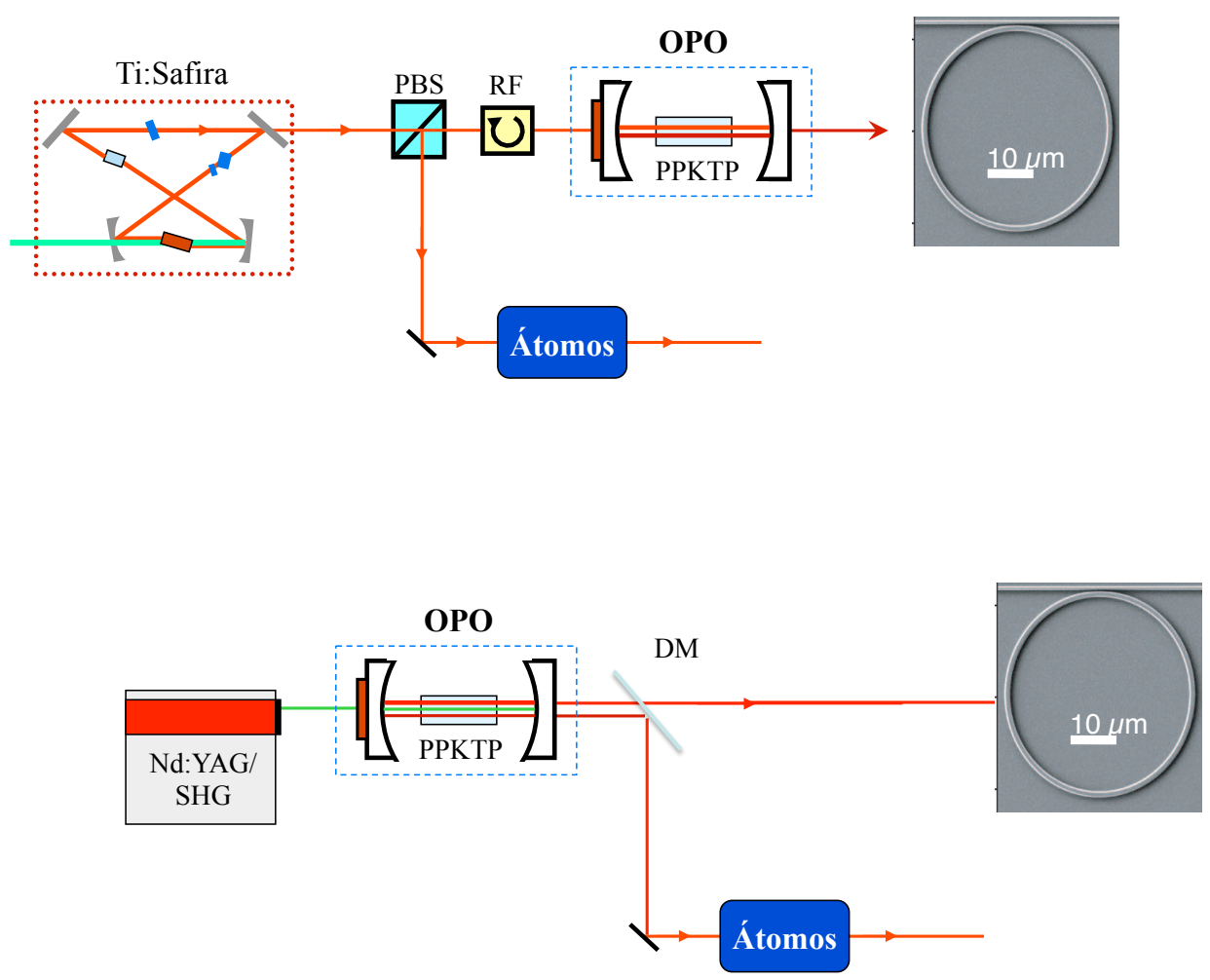

Figura 5.2: (Parte superior) OPO operando com um bombeio em 780nm e gerando feixes sinal e complementar em 1550nm. O feixe de bombeio que é refletido pelo OPO, pode ser acoplado com átomos de Rubídio. Entretanto, o sinal e complementar podem ser acoplados a um micro-ressonador em chip de silício. (b) (Parte inferior) OPO operando com um bombeio em $780 \mathrm{~nm}$ gerando feixes emaranhados, sinal e complementar em 1061nm.

Para finalizar, está em andamento o projeto para geração de campos emaranhados empregando mistura de quatro ondas em células de vapor de Rubídio [24]. Com este sistema também seria possível acoplar campos emaranhados com átomos frios. Assim, nos 
próximos anos, teremos novos resultados desta interface luz-átomos-chips que terá inúmeras aplicações em informação e computação quântica. 


\section{APÊNDICE A}

EXPANSÃO PERTURBATIVA DOS ESPECTROS DE RUÍDO

DOS CAMPOS

Os espectro de ruído são calculados a partir das seguintes expansões:

$$
S_{11}^{I}(\omega)=\alpha_{33}(\omega) \tilde{\Pi}_{33}+\alpha_{44}(\omega) \tilde{\Pi}_{44+}+C_{11}(\omega),
$$

onde o coeficiente complementar $C_{11}$ é definido como

$$
\begin{aligned}
C_{11}(\omega) & =\tilde{\alpha}_{34} \tilde{\Pi}_{34}+\alpha_{55}(\omega) \tilde{\Pi}_{55}+\alpha_{66}(\omega) \tilde{\Pi}_{66} \\
& +\tilde{\alpha}_{56}(\omega) \tilde{\Pi}_{56}+\tilde{\alpha}_{35}(\omega) \tilde{\Pi}_{35}+\tilde{\alpha}_{46}(\omega) \tilde{\Pi}_{46} \\
& +\tilde{\alpha}_{36}(\omega) \tilde{\Pi}_{36}+\tilde{\alpha}_{45}(\omega) \tilde{\Pi}_{45}
\end{aligned}
$$

$\operatorname{com} \tilde{\alpha}_{i j}(\omega)=\alpha_{i j}(\omega)+\alpha_{j i}(\omega)$. Esses termos são relacionados com as contribuições da polarização da outra transição e dos termos cruzados. Como elas contribuem ao espectro é discutido no texto principal.

Da mesma forma, temos o espectro de ruído do segundo campo

$$
S_{22}^{I}(\omega)=\beta_{55}(\omega) \tilde{\Pi}_{55}+\beta_{66}(\omega) \tilde{\Pi}_{66}+C_{22}(\omega),
$$


e coeficientes complementares

$$
\begin{aligned}
C_{22}(\omega) & =\tilde{\beta}_{56} \tilde{\Pi}_{56}+\beta_{33}(\omega) \tilde{\Pi}_{33}+\beta_{44}(\omega) \tilde{\Pi}_{44} \\
& +\tilde{\beta}_{34}(\omega) \tilde{\Pi}_{34}+\tilde{\beta}_{35}(\omega) \tilde{\Pi}_{35}+\tilde{\beta}_{46}(\omega) \tilde{\Pi}_{46} \\
& +\tilde{\beta}_{36}(\omega) \tilde{\Pi}_{36}+\tilde{\beta}_{45}(\omega) \tilde{\Pi}_{45}
\end{aligned}
$$

$\operatorname{com} \tilde{\beta}_{i j}(\omega)=\beta_{i j}(\omega)+\beta_{j i}(\omega)$

E finalmente, para a correlação

$$
\begin{aligned}
S_{12}^{I}(\omega) & =\tilde{\nu}_{35}(\omega) \tilde{\Pi}_{35}+\tilde{\nu}_{46}(\omega) \tilde{\Pi}_{46} \\
& +\tilde{\nu}_{36}(\omega) \tilde{\Pi}_{36}+\tilde{\nu}_{45}(\omega) \tilde{\Pi}_{45}+C_{1 \mid 2}(\omega),
\end{aligned}
$$

$\operatorname{com} \tilde{\nu}_{i j}(\omega)=\nu_{i j}(\omega)+\nu_{j i}(\omega)$ e coeficientes complementares

$$
C_{1 \mid 2}(\omega)=\chi_{11}(\omega)+\chi_{22}(\omega)
$$

definindo

$$
\begin{aligned}
& \chi_{11}(\omega)=\nu_{33}(\omega) \tilde{\Pi}_{33}+\nu_{44}(\omega) \tilde{\Pi}_{44}+\nu \beta_{34}(\omega) \tilde{\Pi}_{34} \\
& \chi_{11}(\omega)=\nu_{55}(\omega) \tilde{\Pi}_{55}+\nu_{66}(\omega) \tilde{\Pi}_{66}+\nu \alpha_{56}\left(\omega \tilde{\Pi}_{56} .\right.
\end{aligned}
$$

Cabe notar que os elementos $\tilde{\Pi}_{33}, \tilde{\Pi}_{44}$ e $\tilde{\Pi}_{34}$, agrupados pelo termo $\chi_{11}(\omega)$, descrevem a resposta atômica com respeito ao campo $\mathbf{E}_{1}$, pois são proporcionais à absorção $\operatorname{Im} p_{1}$ e dispersão Re $p_{1}$. Entretanto, os elementos $\tilde{\Pi}_{55}, \tilde{\Pi}_{66}$ e $\tilde{\Pi}_{56}$, que fazem parte do elemento $\chi_{11}(\omega)$, representam a resposta atômica associada ao campo $\mathbf{E}_{2}$ devido à sua dependencia com $\operatorname{Im} p_{2}$ e Re $p_{2}$. Finalmente, os termos cruzados $\tilde{\Pi}_{35}, \tilde{\Pi}_{46}, \tilde{\Pi}_{36}$ e $\tilde{\Pi}_{45}$ descrevem os principais elementos que contribuem para a correlação $C(\omega)$. 


\section{APÊNDICE B}

ANÁLISE TEÓRICA DA DINÂMICA DE UM SISTEMA DE CINCO NÍVEIS

Com intuito de descrever as longas escalas de tempo da correlação e transmissão obtidas experimentalmente, analisamos de forma teórica a evolução temporal de um sistema de cinco níveis, que representa o sistema de níveis mais próximo daquele que temos no experimento, para determinar qual é a escala de tempo em que o sistema atinge o estado estacionário.

Consideremos o sistema de cinco níveis da figura 4.15.(b) onde os dois campos de prova $\sigma+\left(\mathbf{E}_{1}\right)$ e $\sigma-\left(\mathbf{E}_{2}\right)$ acoplam as transições $|1\rangle \rightarrow|3\rangle$ e $|2\rangle \rightarrow|3\rangle$ respectivamente, e o acoplamento do campo de rebombeio que a acopla efetivamente ${ }^{1}$ a transição $|5\rangle \rightarrow|4\rangle$. O hamiltoniano de interação pode ser construído a partir da expressão geral (2.12) obtida no capitulo 2. Como foi mostrado na seção 4.3.1, o efeito de assimetria espectral no espectro de correlação está relacionado com as forças de espalhamento da luz sobre os átomos e implica num efeito dinâmico da correlação e da transmissão. Neste apêndice, queremos simplesmente analisar a evolução dinâmica da transmissão de um sistema de cinco níveis, pois a sua escala de tempo é a mesma que a da correlação. Portanto não precisamos resolver a dinâmica de uma equação de Langevin, e sim uma equação de Bloch normal, com a qual poderemos ter noção

\footnotetext{
${ }^{1}$ Consideramos a frequência de Rabi efetiva associada ao acoplamento dos níveis hiperfinos nesta transição.
} 
da escala de tempo.

Dado o hamiltoniano, a dinâmica das populações atômicas é descrita pelas equações de Bloch obtidas a partir da eq.(2.17), tal que em forma matricial temos

$$
d \mathbf{x}(t)=-\mathbf{A}_{5 N} \mathbf{x}(t)+\mathbf{x}_{0}
$$

onde o vetor $\mathbf{x}(t)$ contém todas as populações e coerências atômicas, e a matriz contém todos os parâmetros da interação entre os átomos e a luz. Esta equação dinâmica toma a mesma forma da equação para um sistema de três níveis, como foi já revisado no capítulo 3. Para verificar que o sistema de cinco níveis apresenta as principais características dos feixes de prova em EIT para um sistema de três níveis, a figura B.1 mostra a solução estacionária dos principais parâmetros do sistema. As figuras B.1.(a) e (b) mostram a absorção e dispersão dos campos de prova, que estão de acordo a solução 3.11.(b) para um sistema de três níveis. A figura mostra B.1.(c) a função de correlação do modelo heurístico e a figura B.1.(d) mostras as populações dos cinco níveis.

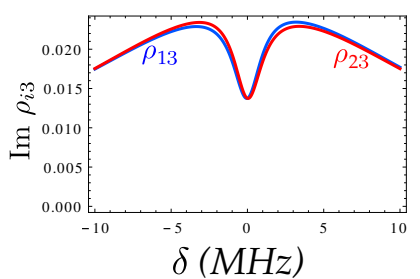

(a)

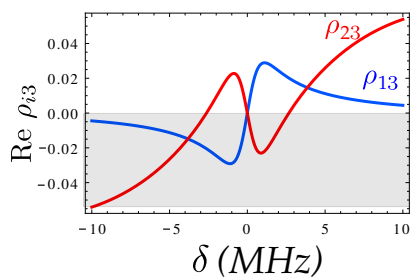

(b)

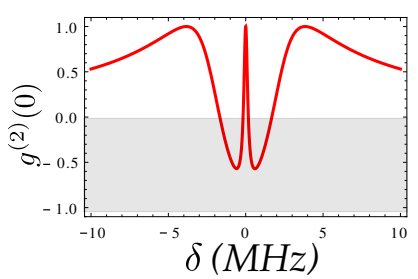

(c)

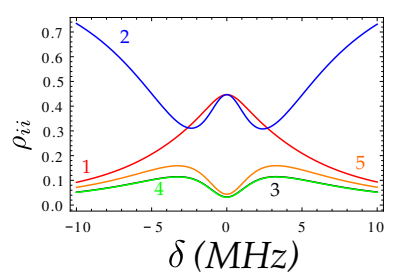

(d)

Figura B.1: (a) Absorção e (b) Dispersão associadas às coerências $\rho_{13}$ e $\rho_{23}$. (c) Função de correlação entre os dois campos de prova determinados a partir das coerências $\rho_{13}$ e $\rho_{23}$ de acordo com o modelo heurístico. (d) Populações atômicas em condição estacionária. Parâmetros do sistema: dessintonia de um fótons $\Delta_{1}=0 \mathrm{MHz}$, dessintonia do rebombeio $\Delta_{\text {Reb }}=0 \mathrm{MHz}, \Omega_{1}=\Omega_{2}=0.3 \Gamma$ para os feixes de prova, decoerência entre os níveis fundamentais $\gamma=0.25 \mathrm{MHz} \Omega_{\text {Reb }}=0.8 \Gamma$ para o rebombeio associada à potência de $930 \mu \mathrm{W}$ retro refletido.

A solução da eq. (B.1) em função do tempo é dada por 


$$
\begin{aligned}
\mathbf{x}(t) & =e^{-A_{5 N} t} \mathbf{x}(0)+\int_{0}^{t} e^{-A_{5 N}\left(t-t^{\prime}\right)} \mathbf{x}_{o} d t^{\prime} \\
& =e^{-A_{5 N} t} \mathbf{x}(0)+A_{5 N}^{-1}\left[e^{-A_{5 N} t}-1\right] \mathbf{x}_{o}
\end{aligned}
$$

A partir desta solução vamos analisar a escala de evolução temporal das populações atômicas para compará-la com a escala temporal obtida no experimento.

\section{B.1 Evolução temporal do sistema de cinco níveis}

Consideramos como condição inicial que a população atômica é preparada no estado $|5\rangle$ de modo que pela normalização dos estados $\rho_{55}=1$ e $\rho_{i i}=0$ para $i=1,2,3$ e 4 . Esta suposição é valida porque experimentalmente é nesse estado onde os átomos são resfriados para depois fazermos a espectroscopia. As figuras B.2.(a) e (b) mostram a evolução temporal das populações dos três estados fundamentais para as dessintonias de dois fótons $\delta=0$ e $\delta=7.6 \mathrm{MHz}$ respectivamente. Notamos que a evolução temporal do sistema para o estado estacionário é da ordem de $\mu$ s, enquanto que experimentalmente observou-se uma evolução em $0.3 \mathrm{~ms}$. Ao mudar a dessintonia de dois fótons de $\delta=0$ para $\delta=7.6 \mathrm{MHz}$ deveríamos observar uma evolução mais lenta como foi observado experimentalmente na figura 4.17.(a) para o caso do bombeio ressonante. No entanto a diferença é desprezível para o caso teórico.

(a)

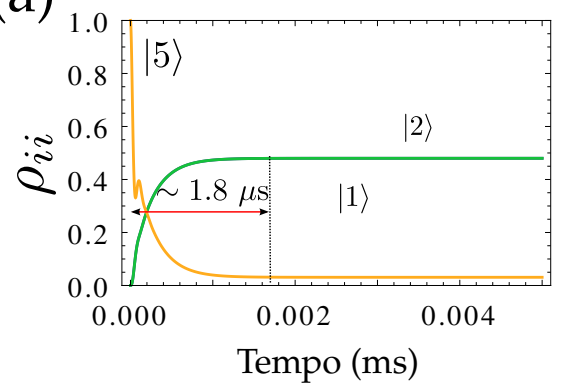

(b)

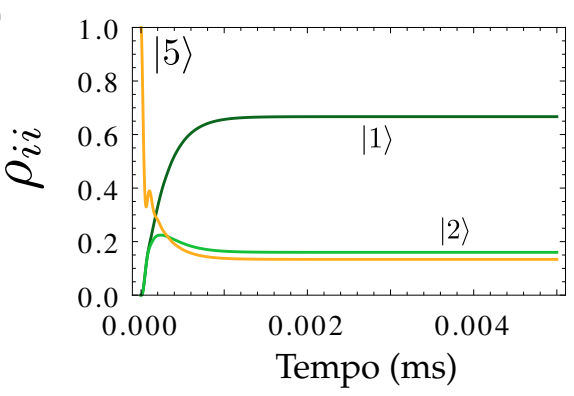

Figura B.2: Evolução temporal das populações atômicas dos níveis fundamentais para (a) $\delta=0.5$ $\mathrm{MHz}$ e $\delta=7.6 \mathrm{MHz}$. Parâmetros do sistema: dessintonia de um fótons $\Delta_{1}=0 \mathrm{MHz}$, dessintonia do rebombeio $\Delta_{\text {Reb }}=0 \mathrm{MHz}, \Omega_{1}=\Omega_{2}=0.3 \Gamma$ para os feixes de prova, decoerência entre os níveis fundamentais $\gamma=0.25 \mathrm{MHz} \Omega_{\text {Reb }}=0.8 \Gamma$ para o rebombeio associada à potência de $930 \mu W$ retro refletido. 
Assim, podemos concluir que a escala de tempo que toma as variáveis internas atômicas para chegar no estado estacionário, em qualquer condição de dessintonia, é da ordem de $10^{-2}$ vezes menor que a observada experimentalmente.

\section{Intensidade do rebombeio}

As figuras B.3.(a) e (b) mostram a evolução temporal da correlação para dois casos de dessintonia de dois fótons $\delta=0.5 \mathrm{MHz}$ e $\delta=7.6 \mathrm{MHz}$, respectivamente, para duas potências do rebombeio. Em linha vermelha se mostra a correlação para uma potência de $P_{R p}=930 \mu \mathrm{W}$, onde o rebombeio é retro refletido e cuja saturação associada é $s=22.70(1)$. Em linha preta se mostra a evolução da correlação para uma potência de $P_{R p}=380 \mu \mathrm{W}$ com saturação de $s=3.78(1)$. Para o caso mais próximo da ressonância de dois fótons $\delta=0.5 \mathrm{MHz}$ o sistema evolui rapidamente para a condição estacionária independente do valor de potência do rebombeio, enquanto que para o caso de dessintonia $\delta=7.6 \mathrm{MHz}$ o sistema evolui num tempo de $\sim 0.3 \mathrm{~ms}$ atingindo diferentes valores de correlação dependendo do valor da potência do rebombeio.
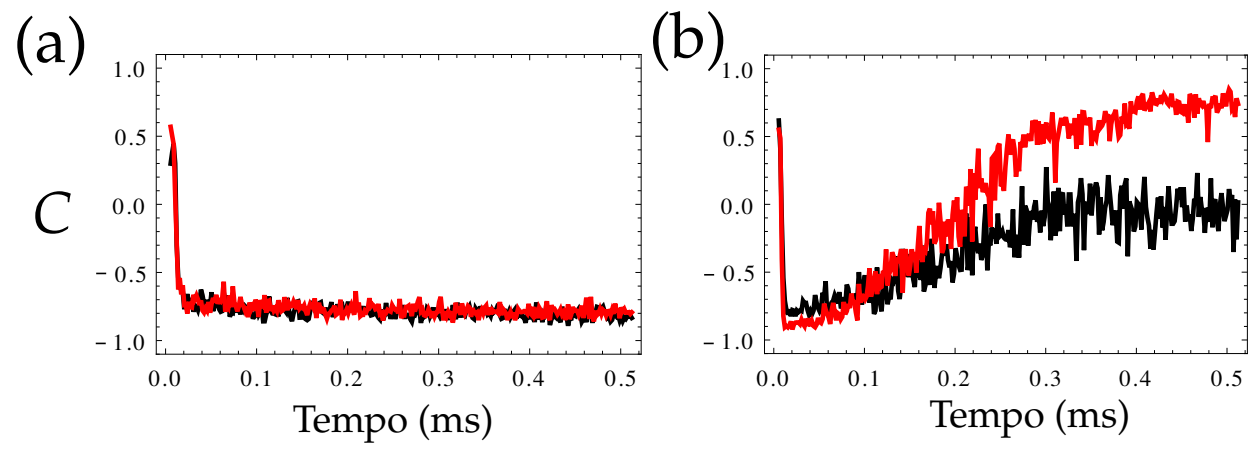

Figura B.3: Evolução temporal do coeficiente de correlação para (a) $\delta=0.5 \mathrm{MHz}$ e (b) $\delta=7.6 \mathrm{MHz}$. Em linha preta traçamos a correlação para um potência do rebombeio $380 \mu \mathrm{W}$ em linha vermelha traçamos a correlação para uma potência do rebombeio de $930 \mu \mathrm{W}$ retro refletido. Parâmetros do sistema: $R=15 \mathrm{MHz} / 0.5 \mathrm{~ms}, \Delta_{R e b}=0 \mathrm{MHz}$.

No que diz respeito ao modelo teórico, analisamos a evolução das populações para as mesmas condições experimentais. As figuras B.4.(a) e (b) mostram as populações dos três 
estados fundamentais $|1\rangle,|2\rangle$ e $|5\rangle$, para os dois casos de dessintonia $\delta=0.5 \mathrm{MHz}$ para $\delta=7.6 \mathrm{MHz}$. Em linha sólida temos as populações para o caso de potência $930 \mu \mathrm{W}$ e em linha tracejada e pontilhada temos as populações para o caso de potência $380 \mu \mathrm{W}$. Em linha pontilhada analisamos o caso de uma potência de $380 \mu \mathrm{W}$ sem retro refletir. Podemos observar que ao diminuir a potência do rebombeio as populações atingem estados estacionários diferentes, porém, a escala de tempo é ainda da mesma ordem de magnitude. Isto mostra uma certa semelhança com o observado experimentalmente na figura B.3.
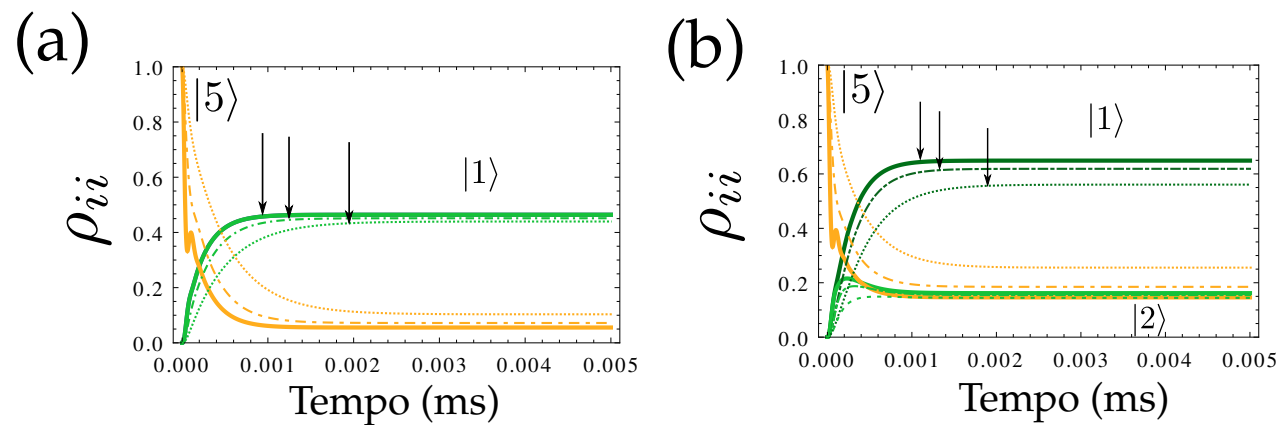

Figura B.4: Evolução temporal das populações atômicas dos níveis fundamentais para (a) $\delta=0.5$ $\mathrm{MHz}$ e $\delta=7.6 \mathrm{MHz}$, com diferentes potencias do rebombeio. As populações são traçadas para potências do rebombeio de $930 \mu \mathrm{W}$ (em linha sólida), de $380 \mu \mathrm{W}$ (em linha tracejada e pontilhada) e de $380 \mu \mathrm{W}$ sem retro refletir (em linha pontilhada). As setas mostram o instante de tempo em que os sistema atinge o estado estacionário. Parâmetros do sistema: dessintonia de um fótons $\Delta_{1}=0 \mathrm{MHz}$, dessintonia do rebombeio $\Delta_{R e b}=0 \mathrm{MHz}, \Omega_{1}=\Omega_{2}=0.3 \Gamma$ para os feixes de prova, decoerência entre os niveis fundamentais $\gamma=0.25 \mathrm{MHz}$.

Portanto o modelo de cinco níveis mostra uma escala de tempo $10^{-2}$ vezes menor à observada experimentalmente. Isto nos leva a supor, que existe algum mecanismo físico com uma escala de tempo característico da ordem de $0.1 \mathrm{~ms}$ que domina a dinâmica atômica. 
[1] H. J. Kimble. The quantum internet. Nature, 453(1023-1030), 2008.

[2] D. F. Phillips, A. Fleischhauer, A. Mair, R. L. Walsworth, and M. D. Lukin. Storage of light in atomic vapor. Phys. Rev. Lett., 86(05), 2001.

[3] I. Novikova, R.L. Walsworth, and Y. Xiao. Electromagnetically induced transparencybased slow and stored light in warm atoms. Nature (London), 6(333-353), 2012.

[4] R. Feynman. Simulating physics with computers. International Journal of Theoretical Physics, 21(6,7), 1982.

[5] J. I. Cirac and P. Zoller. Quantum computations with cold trapped ions. Phys. Rev. Lett., 74(4091), 1995.

[6] Michael A. Nielsen and Isaac L. Chuang. Quantum Computation and Quantum Information. Springer-Verlag, 1st edition, 2000.

[7] Gilbert Grynberg, Alain Aspect, and Claude Fabre. Introduction to Quantum Optics. Cambridge University Press, 1st edition, 2010.

[8] A. Einstein, B. Podolsky, and N. Rosen. Can quantum-mechanical description of physical reality be considered complete? Phys. Rev., 47(777-780), 1935. 
[9] J.S. Bell. on the problem of hidden variable in quantum mechanics. Rev. Mod. Phys., 38(447-452), 1966.

[10] M. Fleischhauer and M.D. Lukin. Dark-state polaritons in electromagnetically induced transparency. Phys. Rev. Lett., 84(22), 2000.

[11] M. Fleischhauer and M.D. Lukin. Quantum memory for photons: Dark-state polaritons. Phys. Rev. A., 65(022314), 2002.

[12] Brian Julsgaard, Jacob Sherson, J. Ignacio Cirac, JaromÃr FiurÃ $\tilde{j}_{i}$ sek, and Eugene S. Polzik. Experimental demonstration of quantum memory for light. Nature, 432(25), 2004.

[13] T. ChaneliÃ"re, D. N. Matsukevich, S. D. Jenkins, S.-Y. Lan, T. A. B. Kennedy, and A. Kuzmich. Storage and retrieval of single photons transmitted between remote quantum memories. Nature, 438(04315), 2005.

[14] Kazuhito Honda, Daisuke Akamatsu, Manabu Arikawa, Yoshihiko Yokoi, Keiichirou Akiba, Satoshi Nagatsuka, , Takahito Tanimura, Akira Furusawa, and Mikio Kozuma. Storage and retrieval of a squeezed vacuum. Phys. Rev. Lett., 100(093601), 2008.

[15] Tigran S. Varzhapetyan, Hebin Li, Gombojav O. Ariunbold, Vladimir A. Sautenkov, Yuri V. Rostovtsev, and Marlan O. Scully. Intensity correlations in a coherently prepared rb vapor in a magnetic field. Opt. Comm., 282(39-43), 2009.

[16] L. S. Cruz, D. Felinto, J. G. Aguirre Gómez, M. Martinelli, P. Valente, A. Lezama, and P. Nussenzveig. Laser-noise-induced correlations and anti-correlations in electromagnetically induced transparency. Eur. Phys. J. D., 41(531-539), 2007.

[17] M. Fleischhauer, U. Rathe, and M. O. Scully. Phase-noise squeezing in electromagnetically induced transparency. Phys. Rev. A., 46(5856), 1992.

[18] K.J. Boller, A. Imamoglu, and S.E. Harris. Observation of electromagnetically induced transparency. Phys. Rev. Lett., 66(2593-2596), 1991. 
[19] H. de Riedmatten, M. Afzelius, M. U. Staudt, C. Simon, and N.Gisin. A solid state light matter interface at the single-photon level. Nature (London), 456(773), 2008.

[20] K. Hammerer, A.S. Sorensen, and E.S.Polzik. Quantum interface between light and atomic ensambles. Rev. Mod. Phys., 82(1041), 2010.

[21] A. S. Coelho, F. A. S. Barbosa, K. N. Cassemiro, A. S. Villar, M. Martinelli, and P. Nussenzveig. Three-color entanglement. Science, 326(823), 2009.

[22] Ling-An $\mathrm{Wu}, \mathrm{Min}$ Xiao, and H. J. Kimble. Squeezed states of light from an optical parametric oscillator. J. Opt Soc. Am. B, 4(10), 1987.

[23] R. C. Pooser, A. M. Marino, V. Boyer, K. M. Jones, and P. D. Lett. Low-noise amplification of a continuous-variable quantum state squeezed states of light from an optical parametric oscillator. Phys. Rev. Lett., 103(010501), 2009.

[24] R. C. Pooser, A. M. Marino, V. Boyer, K. M. Jones, and P. D. Lett. Quantum correlated light beams from non- degenerate four-wave mixing in an atomic vapor: the $\mathrm{d} 1$ and $\mathrm{d} 2$ lines of ${ }^{85} \mathrm{rb}$ and ${ }^{87} \mathrm{rb}$. Opt. Express, 17(19), 2009.

[25] V. Boyer, R. C. Pooser, A. M. Marino, and P. D. Lett. Entangling light in its spatial degrees of freedom with four-wave mixing in an atomic vapor. Chem. Phys. Chme, 10(755760), 2009.

[26] Tony van der Veldt, Jean-Fraqois Roth, Philippe Grelu, and Philippe Grangier. Nonlinear absorption and dispersion of cold rb 87 atoms. Opt. Comm., 137(420-426), 1997.

[27] Stephen E. Harris. Electromagnetically induced transparency. Phys. Today, July(36-42), 1997.

[28] M. Fleischhauer, A. Imamoglu, and J.P. Marangos. Electromagnetically induced transparency: Optics in coherent media. Rev. Mod. Phys., 77(2), 2005. 
[29] O.Kocharovskaya and Ya.I.Khanin. Population trapping and coherent bleaching of a three-level medium by a periodic train of ultrashort pulses. Sov. Phys. JETP, 63(945), 1986.

[30] N. Sangouard, C. Simon, H. de Riedmatten, and N.Gisin. Quantum repeaters based on atomic ensembles and linear optics. Rev. Mod. Phys., 83(33-80), 2011.

[31] L.M. Duan, M.D. Lukin, J.I. Cirac, and P. Zoller. Long-distance quantum communication with atomic ensembles and linear optics. Nature, 414(413-8), 2001.

[32] P. R. Hemmer and M. G. Prentiss. Coupled-pendulum model of the stimulated resonance raman effect. J. Opt Soc. Am. B, 5(8):1613-1623, 1988.

[33] C. L. Garrido Alzar, M. A. G. Martinez, and P. Nussenzveig. Classical analog of electromagnetically induced transparency. Am. J. Phys., 70(37), 2002.

[34] C. L. Garrido Alzar, L.S.Cruz, J. G. Aguirre Gomez, M. França Santos, and P. Nussenzveig. Super-poissonian intensity fluctuations and correlations between pump and probe fields in electromagnetically induced transparency. Europhys. Lett., 61(4,pp. 485491), 2003.

[35] T. Yabuzaki, T. Mitsui, and U. Tanaka. New type of high-resolution spectroscopy with a diode laser. Phys. Rev. Lett., 67(2453), 1991.

[36] Gombojav O. Ariunbolda, Yuri V. Rostovtsevc, Vladimir A. Sautenkova, and Marlan O. Scully. Intensity correlation and anti-correlations in coherently driven atomic vapor. J. Mod. Opt., 57(14-15), 2010.

[37] Vladimir A. Sautenkov, Yuri V. Rostovtsev, and Marlan O. Scully. Switching between photon-photon correlations and raman anticorrelations in a coherently prepared $\mathrm{rb}$ vapor. Phys. Rev. A., 72(065801), 2005. 
[38] M. Martinelli, P. Valente, H. Failache, D. Felinto, L. S. Cruz, P. Nussenzveig, and A. Lezama. Noise spectroscopy of non-linear magneto optical resonances in rb vapor. Phys. Rev. A., 69(043809), 2004.

[39] A. Lezama, P.Valente, H. Failache, M. Martinelli, and P. Nussenzveig. Numerical investigation of the quantum fluctuations of optical fields transmitted through an atomic medium. Phys. Rev. A., 77(013806), 2008.

[40] Yanhong Xiao, Tun Wang, Maria Baryakhtar, Mackenzie Van Camp, Michael Crescimanno, Michael Hohensee, Liang Jiang, David F. Phillips, Mikhail D. Lukin, Susanne F. Yelin, and Ronald L. Walsworth. Electromagnetically induced transparency with noisy lasers. Phys. Rev. A., 80(041805(R)), 2009.

[41] Steven Chu, L. Hollberg, J. E. Bjorkholm, Alex Cable, and A. Ashkin. Threedimensional viscous confinement and cooling of atoms by resonance radiation pressure. Phys. Rev. Lett., 55(48-51), 1985.

[42] A. Aspect, J. Dalibard, A. Heidmann, C. Salomon, and C. Cohen-Tannoudji. Cooling atoms with stimulated emission. Phys. Rev. Lett., 57(1688-1691), 1986.

[43] D. Felinto, L. S. Cruz, R. A. de Oliveira, H. M. Florez, M. H. G. de Miranda, P. Nussenzveig, M. Martinelli, and J. W. R. Tabosa. Physical interpretation for the correlation spectra of electromagnetically-induced-transparency resonances. Opt. Express, 24(1512), 2013.

[44] H. M. Florez, L. S. Cruz, M. H. G. de Miranda, R. A. de Oliveira, J.W. R. Tabosa, M. Martinelli, and D. Felinto. Power-broadening-free correlation spectroscopy in cold atoms. Phys. Rev. A., 88(033812), 2013.

[45] John David Jackson. Classical electrodynamics. Wiley, 3rd edition, 1999.

[46] Marcis Auzinsh, Dmitry Budker, and Simon Rochester. Optically Polarized Atoms Understanding light-atom interactions. Oxford University Press, 1st edition edition, 2010. 
[47] Hans-A. Bachor and Timothy C. Ralph. A guide to experiments in Quantum optics. Wiley, 2nd edition, 2004.

[48] Rodney Loudon. The quantum theory of light. Clarendon Press-Oxford, 2da edition, 1983.

[49] Marlan O. Scully and M. Suhail Zubairy. Quantum Optics. Cambridge University Press, 2nd edition, 2001.

[50] R Hanbury Brown and R Twiss. Correlation between photons in two coherent beams of light. Nature, January 7, 1956.

[51] Leonarl Mendel and Emil Wolf. Optical Coherence and quantum optics. Cambridge University Press, 1era ed. edition, 1995.

[52] C.W.Gardiner. Handbook of Stochastic Methods. Springer-Verlag, 1990.

[53] Felippe Alexandre Silva Barbosa. Robustez do emaranhamento em variáveis contínuas e fotodetecção de feixes intensos no domínio temporal. PhD thesis, Universidade de São Paulo, 2013.

[54] Tânia Tomé e Mario Jose Oliveira. Dinâmica estocástica e Irreversibilidade. EDUSP, 1era edition, 2001.

[55] Robert Brown. A brief account of microscopical observations made in the months of june, july and august, 1827, on the particles contained in the pollen of plants; and on the general existence of active molecules in organic and inorganic bodies. Phil. Mag., $4(161), 1827$.

[56] A. Einstein. Investigations on the theory of, Brownian movement. Dover Publications Inc., 2nd edition, 1956.

[57] N.G. Van Kampen. Stochastic processes in Physics and Chemistry. Elsevier, 3ra ediÃ£̃̃£o edition, 2007.

[58] D.F Walls and G.J. Milburn. Quantum Optics. Springer, 2nd edition, 2008. 
[59] M.D. Lukin. Colloquium: Trapping and manipulating photon states in atomic ensembles. Rev. Mod. Phys., 75(April), 2003.

[60] D. Leibfried, R. Blatt, C. Monroe, and D. Wineland. Quantum dynamics of single trapped ions. Rev. Mod. Phys., 75(281-324), 2003.

[61] R. Miller, T. E. Northup, K.M. Birnbaum, A. Boca, A. D. Boozer, and H. J. Kimble. Trapped atoms in cavity qed: coupling quantized light and matter. J .Phys. B: At. Mol. Opt. Phys., 38(S551-S565), 2005.

[62] Robert W. Boyd. Nonlinear optics. Academic Press, 2nd edition, 2003.

[63] A. E. Kozhekin, K. Molmer, and E. Polzik. Quantum memory for light. Phys. Rev. A., 62(033809), 2000.

[64] C. H. van der Wal, M. D. Eisaman, A. Andre, R.L. Walsworth, D. F. Phillips, A.S. Zibrov, and M. D. Lukin. Atomic memory for correlated photon states. Science, 301(196), 2003.

[65] M. Fleischhauer, A Imamoglu, and J. P. Marangos. Electromagnetically induced transparency: Optics in coherent media. Rev. Mod. Phys., 77(633), 2005.

[66] J.-F. Roch, K. Vigneron, Ph. Grelu, A. Sinatra, J. Ph. Poizat, and Ph. Grangier. Quantum nondemolition measurements using cold trapped atoms. Phys. Rev. Lett., 78(634-637), 1997.

[67] J. X. Wu, Chengjie Zhu, and Y. P. Yang. Dynamic lossless polarization gate using a coherently prepared atomic medium. Opt. Lett., 40(4975-78), 2015.

[68] H.J. Metcalf and P. Straten. Laser Cooling and Trapping. Springer-Verlag, 1st edition, 1999.

[69] Cristopher J. Foot. Atomic Physics. Oxford University Press, 1st edition, 2005.

[70] William D. Phillips. Laser cooling and trapping of neutral atoms. Nobel Lecture, December 8, 1997. 
[71] Alzetta G., A. Gozzini, L. Moi, and G. Orriols. An experimental method for the observation of r.f. transitions and laser beat resonances in oriented na vapour. Nuovo Cimento Soc. Ital. Fis., B 36(5), 1976.

[72] S. E. Harris, J. E. Field, , and A. Kasapi. Dispersive properties of electromagnetically induced transparency. Phys. Rev. A., 46(R29), 1992.

[73] L. Hau, S. Harris, Z. Dutton, and C.H. Behroozi. Light speed reduction to 17 metres per second in an ultracold atomic gas. Nature, 397(594), 1999.

[74] Warren Nagourney. Quantum Electronics for Atomic Physics. Oxford University Press, 1st edition.

[75] R. Waiser and P. Zoller. Laser-noise-induced polarization fluctuations as a spectroscopic tool. Phys. Rev. A., 49(5067-5077), 1994.

[76] J. C. Camparo and R. P. Frueholz. Observation of the rabi resonances spectrum. Phys. Rev. A., 38(6143-50), 1988.

[77] Aldo Godone, Salvatore Micalizio, and Filippo Levi. Rabi resonances in the $\lambda$ excitation scheme. Phys. Rev. A., 66(063807), 2002.

[78] A. F. Huss, R. Lammegger, C. Neureiter, E.A. Korsunsky, and L.Windholz. Phase correlation of laserwaves with arbitrary frequency spacing a. Phys. Rev. Lett., 93(223601), 2003.

[79] M. Jain. Excess noise correlation using population-trapped atoms. Phys. Rev. A., 50(1899), 1994.

[80] Jacques Dupont-Roc, Claude Cohen-Tannoudji, and Gilbert Grynberg. Atom-Photon Interactions - Basic Processes and Applications. John Wiley \& Sons, 1992.

[81] L. Ricci, M. Weidemuller, C. Zimmermann V. Vuletic W. Koenig T. Esslinger, A. Hemmerich, and T. W. Haensch. A compact grating-stabilized diode laser system for atomic physics. Opt. Commun, 177(4), 1995. 
[82] C.J. Hawlthorn, K.P. Weber, and R.E. Scholten. Littrow configuration tunable external cavity diode laser with fixed direction output beam. Rev. Sci. Instrum., 72(12), 2001.

[83] E. A. Donley, T. P. Heavner, F. Levi, M. O. Tataw, and S. R. Jefferts. Double-pass acoustooptic modulator system. Rev. Sci. Instrum., 76(063112), 2005.

[84] Roy J. Glauber. The quantum theory of optical coherence. Phys. Rev., 130(2529-2539), 1963.

[85] C. Cohen-Tannoudji, J. Dupont-Roc, and G. Grynberg. Atom-Photon interaction Basic processes and applications. Wiley, 1st edition, 1992.

[86] Alessandro de Sousa Villar. Emaranhamento multicolor entre feixes intensos de luz. PhD thesis, Universidade de São Paulo, 2007.

[87] Hans Marin Florez. Espectroscopia de correlação de ruído em transparência eletromagneticamente induzida em átomos frios. Master's thesis, Universidade de São Paulo, 2012.

[88] A. Villar. The conversion of phase to amplitude fluctuations of a light beam by an optical cavity. Am. J. Phys., 76(922), 2008.

[89] H. M. Florez, A. Kumar, K. Theophilo, P. Nussenzveig, and M. Martinelli. Correlation spectroscopy in cold atoms: light sidebands resonances in electromagnetically induced transparency condition. arXiv:1508.04858, 2015.

[90] Frank Blatt Thorsten Peters, Benjamin Wittrock and Thomas Halfmann. Thermometry of ultracold atoms by electromagnetically induced transparency. Phys. Rev. A., 85(063416), 2012.

[91] J. Vanier. Atomic clocks based on coherent population trapping: a review. Appl. Phys. $B, 81: 421 \hat{4} 442,2005$.

[92] D. K. Serkland G. M. Peak M. Varghese G. Tepolt J. R. Leblanc R. Lutwak, A. Rashed and M. Mescher. âThe Miniature Atomic Clock-Pre-Production Results. in proceedings of 
TimeNav07: Joint 21th European Frequency and Time Forum (EFTF)\&IEEE International Frequency Control Symposium (IEEE-FCS), ed. (geneva, switzerland, 2007) edition, 2007.

[93] Xihua Yang, Yuanyuan Zhou, and Min Xiao. Entangler via electromagnetically induced transparency with an atomic ensemble. Scientific reports, 3:3479, 2013.

[94] Avik Dutt, Kevin Luke, Sasikanth Manipatruni, Alexander L. Gaeta, Paulo Nussenzveig, and Michal Lipson. On-chip optical squeezing. Phys. Rev. Applied, 3:044005, 2015. 